\title{
Decoupling and integrability of nonassociative vacuum phase space gravitational equations with star and R-flux parametric deformations
}

\author{
Elşen Veli Veliev * \\ Department of Physics, Kocaeli University, 41380, Izmit, Turkey \\ Laurenţiu Bubuianu ${ }^{\dagger}$ \\ SRTV - Studioul TVR Iaşi and University Appolonia, 2 Muzicii street, Iaşi, 700399, Romania \\ Sergiu I. Vacaru $\ddagger$ \\ Physics Department, California State University at Fresno, Fresno, CA 93740, USA; \\ and Dep. Theoretical Physics and Computer Modelling, \\ Yu. Fedkovych Chernivtsi National University, 101 Storozhynetska street, Chernivtsi, 58029, Ukraine
}

March 10, 2021

\begin{abstract}
We prove that nonassociative star deformed vacuum Einstein equations can be decoupled and integrated in certain general forms on phase spaces involving real R-flux terms induced as parametric corrections on base Lorentz manifold spacetimes. The geometric constructions are elaborated with parametric (on respective Planck, $\hbar$, and string, $\kappa:=\ell_{s}^{3} / 6 \hbar$, constants) and nonholonomic dyadic decompositions of fundamental geometric and physical objects. This is our second partner work on elaborating nonassociative geometric and gravity theories with symmetric and nonsymmetric metrics, (non) linear connections, star deformations defined by generalized Moyal-Weyl products, endowed with quasi-Hopf algebra, or other type algebraic and geometric structures, and all adapted to nonholonomic distributions and frames. We construct exact and parametric solutions for nonassociative vacuum configurations (with nontrivial or effective cosmological constants) defined by star deformed generic off-diagonal (non) symmetric metrics and (generalized) nonlinear and linear connections. The coefficients of geometric objects defining such solutions are determined by respective classes of generating and integration functions and constants and may depend on all phase space coordinates [spacetime ones, $\left(x^{i}, t\right)$; and momentum like variables, $\left(p_{a}, E\right)$ ]. Quasi-stationary configurations are stated by solutions with spacetime Killing symmetry on a time like vector $\partial_{t}$ but with possible dependencies on momentum like coordinates on star deformed phase spaces. This geometric techniques of decoupling and integrating nonlinear systems of physically important partial differential equations (the anholonomic frame and connection deformation method, AFCDM) is applied in our partner works for constructing nonassociative and locally anisotropic generalizations of black hole and cosmological solutions and elaborating geometric flow evolution and classical and quantum information theories.
\end{abstract}

Keywords: exact and parametric solutions; nonassociative geometry; R-flux non-geometric background; nonholonomic star deformations; nonsymmetric metrics; nonlinear connections.

*email: elsen@kocaeli.edu.tr and elsenveli@hotmail.com

${ }^{\dagger}$ email: laurentiu.bubuianu@tvr.ro

$\ddagger$ email: sergiu.vacaru@gmail.com

Address for post correspondence in 2020-2021 as a visitor senior researcher at YF CNU Ukraine is: Yu. Gagarin street, 37-3, Chernivtsi, Ukraine, 58008; authors' order reflects certain chronology of former and futures research programs and involves equal contributions in obtaining new results in this work 


\section{Contents}

1 Introduction $\quad 3$

2 Nonholonomic dyadic decompositions and nonassociative star products 5

2.1 Nonlinear connections for dyadic splitting in commutative phase spaces . . . . . . . . . . . 6

2.1.1 Conventions on dyadic local coordinates on phase spaces and base spacetimes . . . . . 6

2.1.2 Nonlinear connections and dyadic splitting . . . . . . . . . . . . . . 7

2.2 Nonassociativity, dyadic N-connections, and quasi-Hopf algebras . . . . . . . . . . . . . . . 9

2.2.1 Nonassociative star product and dyadic s-structures . . . . . . . . . . . . . . . . . 9

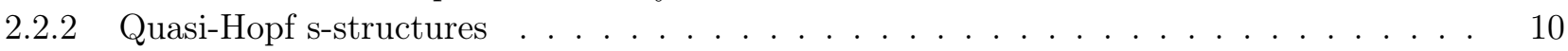

2.3 Forms and d-tensors for quasi-Hopf s-adapted structures . . . . . . . . . . . . . . . . 12

2.3.1 Nonassociative dyadic deformations of star exterior products . . . . . . . . . . 12

2.3.2 s-tensor and dyadic quasi-Hopf star deformations . . . . . . . . . . . . . . . 14

3 Dyadic geometry of star deformed (non) symmetric s-metrics 14

3.1 The geometry of phase space s-metrics and canonical s-connections . . . . . . . . . . . . 14

3.1.1 Symmetric and nonsymmetric s-metric structures and star deformations . . . . . . . 15

3.1.2 Canonical s-connections as distortions of dyadic LC-connections . . . . . . . . . . . . 16

3.1.3 Canonical nonholonomic Ricci and Einstein s-tensors . . . . . . . . . . . . . . 17

3.2 Nonassociative canonical s-connections and LC-configurations . . . . . . . . . . . . . . 19

3.2.1 Conventions on parameterizing nonassociative R-flux star deformations . . . . . . . . 19

3.2.2 Nonsymmetric metrics and s-metrics and their inverses . . . . . . . . . . . . . . 20

3.2.3 Star deformed LC-connections and canonical s-connections . . . . . . . . . . . . . . 21

3.3 Nonassociative parametric decompositions of canonical Riemann and Ricci s-tensors . . . . 23

3.3.1 Star and parametric deformed canonical curvature s-tenors . . . . . . . . . . . . 23

3.3.2 Parametric decomposition of star canonical Ricci s-tensors and their distortions . . . . 24

4 Nonassociative nonholonomic dyadic vacuum Einstein equations 25

4.1 Nonassociative vacuum Einstein equations with (non) symmetric s-metrics . . . . . . . . . . 25

4.1.1 Parametric decomposition of nonsymmetric star deformed s-metrics . . . . . . . . . 26

4.1.2 Star deformed canonical s-adapted nonassociative vacuum gravitational equations . . . 27

4.2 Vacuum gravitational equations with effective nonassociative s-sources . . . . . . . . . . . 29

4.2.1 Dyadic parametrization of effective nonassociatvie s-sources . . . . . . . . . . . . 29

4.2.2 Computing parametric R-flux induced (non) symmetric metrics and effective source . . 30

4.3 Adapted lifts and horizontal projections of nonassociative canonical Ricci s-tensors . . . . . 31

4.4 First order parametric nonassociative s-adapted spacetime corrections . . . . . . . . . . . 34

5 Decoupling \& integrability of nonassociative vacuum gravitational equations 36

5.1 Off-diagonal ansatz for phase space parametric s-metrics . . . . . . . . . . . . . . 36

5.1 Quasi-stationary ansatz for s-metrics and R-flux sources . . . . . . . . . . . . . . 37

5.1 .2 Nonassociative and parametric canonical Ricci s-tensors . . . . . . . . . . . . 38

5.2 Decoupling of nonassociative nonholonomic stationary vacuum Einstein equations . . . . . . . 39

5.3 Generating stationary solutions for nonassociative vacuum phase spaces . . . . . . . . . . 40

5.3.1 Off-diagonal solutions with R-flux induced sources and noholonomic s-torsion . . . . . 40

5.3.2 Computing stationary coefficients for (non)symmetric star deformed s-metrics . . . . . 42

5.3.3 Extracting nonassociative parametric LC-configurations . . . . . . . . . . . . . 43

5.4 Nonassociative nonlinear symmetries for stationary generating functions and R-flux sources . 45 
A Nonassociative differential geometry with s-connections \& nonsymmetric metrics 49

A.1 Dyadic s-adapted linear connections and quasi-Hopf s-structures . . . . . . . . . . . . . . 49

A.2 Star generalizations of the torsion, Riemann and Ricci s-tensors . . . . . . . . . . . 51

A.2.1 Nonassociative star s-torsions for s-connections and quasi-Hopf s-structures . . . . . . 51

A.2.2 Star deformed dyadic curvature of quasi-Hopf s-adapted structures . . . . . . . . . 52

A.3 Nonassociative s-metrics and canonical s-connections with small parameters . . . . . . . . . 55

A.3.1 Parametric decomposition of inverse nonsymmetric star s-metrics . . . . . . . . . 56

A.3.2 Nonholonomic dyadic parametric decomposition of star canonical s-connections . . . . 56

A.3.3 Parametric coefficients for the nonassocative canonical Ricci s-tensor . . . . . . . . 58

B Nonholonomic dyadic deformations into parametric solutions $\quad 60$

B.1 Using s-metric coefficients as generating functions . . . . . . . . . . . . . . . . . . 60

B.2 Polarization parametric functions for nonassociative prime and target s-metrics . . . . . . . 61

B.3 Parametric nonassociative transforms to quasi-stationary s-metrics . . . . . . . . . . . . 62

\section{Introduction}

There were formulated consistent approaches to nonassociative geometry and gravity induced by the R-flux background of string theory [1] and, for constructions with quasi-Hopf algebras, 2] (see references therein). The formalism was developed up to a level with nonassociative star deformed vacuum Einstein equations on phase space $\mathcal{M}=T_{\|}^{*} V$, with proofs that there are nontrivial R-flux contributions resulting in real distortions of the Ricci tensor for the Levi-Civita connection on Lorentz type spacetime manifold $V 1$ That research was performed for decompositions of (non)associative geometric and physical objects (such as symmetric and nonsymmetric metrics, generalized (non) linear connections and their torsion and curvature, etc.) up to linear orders on parameters $\hbar$ (Planck constant), $\kappa$ (string constant), which can be proportional to the complex unity, and with nontrivial $\hbar \kappa$ real terms. It was emphasized that for further developments it would be very important to study various features of nonassociative models of gravity and matter field interactions when star deformed Einstein equations are defined on phase spaces and projected to spacetime manifolds, and to find explicit solutions of such physically important nonlinear systems of nonassociative partial differential equations, PDEs.

In our first partner work on nonassociative nonholonomic geometry and gravity [3], we noted that because of generic nonlinearity and tensor coupling of (non) associative / commutative gravitational equations, even for vacuum configurations, the solutions of respective systems of PDEs can not be constructed in general off-diagonal exact or parametric forms depending on some phase and spacetime variables if we work only in holonomic variables determined with respect to local coordinate frames and for diagonalizable metrics. In such theories, R-flux star deformations extend the geometric approach to constructions on phase spaces modelled as (co) tangent bundles endowed with symmetric and nonsymmetric metrics and generalized connection structures depending both on spacetime and momentum like coordinates. Similar methods were studied in the geometry of algebraic structures, metrics, and nonlinear and distinguished connections adapted to nonholonomic

\footnotetext{
${ }^{1}$ See in next section details on index, coordinatesand frames conventions. Here we note that we shall use local real spacetime and complexified co-fiber, momentum like, coordinates " $u=(x, " p)=\left\{{ }^{\prime \prime} u^{\alpha}=\left(u^{k}=x^{k}\right.\right.$, " $\left.p_{a}=\left(i \hbar^{-1} p_{a}\right)\right\}$, for complex unity $i^{2}=-1$, where left up or low labels " "" will be used for geometric objects and spaces with dual complexified momentum like coordinates; and coordinates $x=\left(x^{i}\right)=\left(x^{1}, x^{2}, x^{3}, x^{4}=t\right)$ on a four dimensional, 4-d, Lorentz manifold with signature of metric $(+++-)$; for simplicity; in this paper, we work with 4-d basic manifolds and 8-d (co) tangent bundles (i.e. phase spaces); we can consider in similar forms spaces of higher dimension when the base spacetime or phase spaces are of dimension 5 , or 6 , and respective total dimension 10,11,12; if necessary, with certain imbedding and/or compactification of coordinates.
} 
distributions (in brief, N- and d-connections). Such a formalism was developed in modified Finsler-LagrangeHamilton geometry, see recent reviews and applications in physics in [4, 5], and in generalized EinsteinEisenhart-Moffat gravity extended for nonholonomic Finsler like variables [6, 7, 8, , 9, 10, 11, 12, 13, 14, 15, 16, To perform rigorous mathematical formulations of nonassociative gravity and geometric flow theories on total phase spaces and elaborate on methods of constructing exact and parametric physically important solutions is not possible if we work only in local coordinate bases and use star products stated only for partial derivatives and adapting the geometric objects to respective (linear) nonassociative / noncommutative / commutative algebraic structures. It is important to apply a more advanced geometric techniques involving nonassociative and noncommutative nonholonomic (i.e. non-integrable, equivalently, anholonomic) distributions and frames, together with nonholonomic deformations of (non) linear connections and respective N-connection and parametric decompositions of (non) symmetric structures and related distortions of fundamental geometric objects (for instance, of the torsion and curvature tensors, distorted Ricci tensors etc.).

This work provides a nonholonomic dyadic formulation of nonassociative geometry and vacuum gravity when a decomposition on so-called "shell by shell" two dimensional variables determined by respective Nadapted frames (in brief, s-structures and/or s-decompositions) allows to prove some general decoupling and integrability properties of corresponding systems of nonlinear PDEs. We generalize the approaches toward to the theory of nonassociative gravity from proposed in [1, 2] to a formalism when the geometric constructions are performed following the method of star nonholonomic deformations elaborated in [3]. Our main goal is to write the nonassociative vacuum gravitational equations in certain adapted nonholonomic variables with conventional $2+2+2+\ldots$ splitting of dimensions and in terms of auxiliary distinguished connection:2 and, in result, to generate new classes of exact and parametric physically important solutions. The zero torsion Levi-Civita, LC, configurations can be extracted by imposing additional nonholonomic constraints. Following the nonholonomic dyadic approach, we can work on the entire phase space and formulate nonassociative gravitational field equations using the geometric formalism developed and outlined for commutative and noncommutative spaces in [15, 17, 18, 4, 5].

For simplicity, in this paper, we restrict our considerations only to nonassociative nonholonomic stationary vacuum configurations which can be characterized also by nontrivial and/or effective cosmological constants encoding or constraining nonholonomically the contributions of R-flux terms. The stationary condition means that the metrics, connections, and related fundamental geometric objects are with Killing symmetry on a vector $\partial_{4}=\partial_{t}$ for a time like spacetime coordinate $x^{4}=t$. In our partner works, we elaborate on general methods for constructing exact and parametric stationary and cosmological type solutions (when the dependence on a time like variable is crucial) of nonassociative modified Einstein equations with nontrivial and/or effective matter sources and for generalized/ relativistic geometric flow evolution equations. Such a geometric techniques is called in our works as the anholonomic frame and connection deformation method, AFCDM, 3 of constructing exact solutions in (non) commutative/ supersymmetric/ string / massive and other type modified gravity and general relativity, GR, theories was developed in [19, 20, 21, see also references therein, on possible applications in modern cosmology and astrophysics, geometric thermodynamics and classical and quantum information flow theories [22, 23]. Here we note that our nonholonomic dyadid 4 approach to generating exact solutions in (modified) gravity theories is more general and different from other type methods with dyadic decompositions (for instance, with the Newmann-Penrose formalism, see a review of geometric methods and main results in [24, 25, 26, 27]). Applying the AFCDM, we work with distortions of connection structures which allow to generate generic off-diagonal solutions depending on all spacetime and phase space coordinates. This is not possible if we consider only the LC-connection and other dyadic/ triad/ tetrad etc. methods.

\footnotetext{
${ }^{2}$ d-connections, adapted to N-connection structures; for dyadic decompositions, we use also the term s-connection

3 in some previous works, we wrote in brief AFDM

${ }^{4}$ in mathematical and physical literature, there are used equivalently both terms "dyad" and "diad" (the last one is from a corresponding Latin word); in our works, such terms are introduced for conventional nonholonomic decompostions of spaces and respective indices of geometric objects into certain shells, s, of dimension 2 ; in this paper, we write "dyad" even in some our previous articles it was used the term "diad".
} 
It should be noted that algebras and geometries with nonassociative structure arise from various developments on quantum mechanics with magnetic monopole, string and M-theory with non-geometric fluxes etc., see [28, 29, 30] for reviews of such approaches. Here we cite also a series of nonassociative theories with octonionic star products and variables [31, 32, 33, 34, 35, 36], where the AFCDM can be applied for generating exact solutions and performing deformation quantization, but we omit to study such models in this paper.

This work is structured as follows: Section 2 provides a dyadic formalism for the geometry of nonassociative nonholonomic star products and deformations on phase spaces modelled as nonholonomic (co) tangent Lorentz bundles. There are defined the N-connection and related frame and dual frame structure with shell by shell decomposition (s-decomposition). The nonholonomic dyadic constructions are considered for (non) associative phase spaces and with projections to spacetime components involving R-flux contributions.

Main features of star s-deformed nonsymmetric and symmetric metrics and related nonassociative canonical s-connections and constraints for torsionless LC-configurations are considered in section 3 It is formulated a model of nonassociative dyadic geometry of phase spaces and spacetime (non) symmetric metrics with canonical s-connection.

Section 4 is devoted to elaborating a dyadic formalism with nonholonomic $(2+2)+(2+2)$ splitting for nonassociative s-decomposition of vacuum Einstein equations encoding nontrivial and polarized cosomological constants and/or sources induced by R-fluxes and parametric star deformations of geometric objects. We follow the methods of nonassociative differential geometry from section 3 of [3] re-defined in s-adapted variables at the end of this paper in appendix $\mathrm{A}$. There are considered s-variables for which the nonsymmetric components of Ricci s-tensors are related to parametric and s-decompositions of the nonsymmetric parts of s-metrics. Such nonassociative geometric constructions involve complex (non) symmetric s-metric structures and canonical Nand s-connections.

Section 5 contains a further development of the AFCDM for nonassociative vacuum gravity. There are stated and analyzed the conditions on general decoupling and integrability of star nonholonomic deformed vacuum Einstein equations. We show how corresponding systems of nonlinear PDEs spit into coupled "shell by shell" systems of two plus two etc. equations with coefficients depending recurrently on respective spacetime and phase coordinates. Then we prove that such nonlinear s-systems are with a general decoupling property and can be integrated in general form for general off-diagonal ansatz for stationary and phase spaces Killing symmetries, for instance, on $\partial_{t}$ and $\partial_{8}=\partial_{E}$. We study an important class of nonassociative nonlinear symmetries relating generation functions and generating sources connected to R-flux star deformations and encoded into effective shell cosmological constants. The nonassociative AFCDM is elaborated in such a form which is adapted to s-structures and parametric decompositions involving symmetric and nonsymmetric components of s-metrics and s-connections.

Conclusions are presented in section 6. In appendix A, there are provided main definitions and formulas for nonassociative s-distinguished linear connections, s-connections, and introduced associated covariant derivatives with formulas for computing s-components of Riemannian, torsion and Ricci s-tensors. In appendix[B, we generalize for nonassociative vacuum gravity the procedure of generating quasi-stationary solutions elaborated for associative and commutative phase spaces in section 5 of [40]. We show how to construct nonholonomic dyadic and parametric deformations of phase space metrics into (non) associative / commutative phase space and spacetime configurations.

\section{Nonholonomic dyadic decompositions and nonassociative star products}

In the first partner work [3], the nonassociative vacuum Einstein equations from [1, 2] were generalized and written in canonical nonholonomic variables on a phase space modeled as a cotangent Lorentz bundle $\mathcal{M}=$ $T_{\|}^{*} \mathbf{V}$ enabled with nonlinear connection, N-connection structure " $\mathbf{N}$ and a respectively defined $\mathrm{N}$-adapted star product using general frame transforms. We use boldface indices for spaces and geometric objects enabled with (adapted to) N-connection structure as we introduce below. In this section, we outline the N-connection 
geometry with a splitting of type $4+4$ on $T V$ and a $T^{*} V$ and further nonholonomic dyadic decomposition into oriented shells $s=1,2,3,4$ with conventional splitting of dimensions of type $(2+2)+(2+2)$, in brief called a s-decomposition. This is necessary for developing the anholonomic frame and connection deformation method, AFCDM (on previous results and applications to (non) commutative theories, see [4, 20, 21, 23]), for constructing exact and parametric solutions in nonassociative gravity. Then, we study certain important properties of the nonassociative star products introduced in $\mathrm{N}$-adapted form with s-splitting and quasi-Hopf structure, which generalize the geometric constructions from [2, 3].

\subsection{Nonlinear connections for dyadic splitting in commutative phase spaces}

We summarize necessary concepts and definitions of geometric objects adapted to N-connection structure and s-splitting of relativistic phase spaces with real spacetime and complex velocity/momentum type variables.

\subsubsection{Conventions on dyadic local coordinates on phase spaces and base spacetimes}

The geometric arena for elaborating on (non) associative / commutative geometric and physical models in this work consists from (co) tangent Lorentz bundles with conventional splitting of bases and (co) fiber like coordinates and respective indices. We shall use such notations for local real (spacetime and total phase space) and complex (co) fiber coordinates:

$$
\begin{aligned}
& \text { on } \mathbf{V} \text { and } \quad \begin{aligned}
& \mathbf{V}: \\
x= & \left\{x^{i}\right\}={ }_{s} x=\left\{x^{i_{s}}\right\}=\left(x^{i_{1}}, x^{a_{2}} \rightarrow y^{a_{2}}\right)=\left(x^{i_{2}}\right), \text { with } x^{4}=t, \\
& \text { where } i, j, \ldots=1,2,3,4 ; \text { shells : } s=1: \text { when } i_{1}, j_{1}, \ldots=1,2 ; s=2, a_{2}, b_{2}, \ldots=3,4 ;
\end{aligned}
\end{aligned}
$$

on $T \mathbf{V}$ and $T_{s} \mathbf{V}$ :

$$
\begin{aligned}
& u=(x, y)=\left\{u^{\alpha}=\left(u^{k}=x^{k}, u^{a}=y^{a}\right)\right\}= \\
& { }_{s} u=\left({ }_{s} x,{ }_{s} y\right)=\left\{u^{\alpha_{s}}=\left(u^{k_{s}}=x^{k_{s}}, u^{a_{s}}=y^{a_{s}}\right)\right\}=\left(x^{i_{1}}, x^{i_{2}}, x^{a_{3}} \rightarrow y^{a_{3}}, x^{a_{4}} \rightarrow y^{a_{4}}\right) \text {, shells } \\
& s=1,2,3,4 \text {, where } \alpha, \beta, \ldots=1,2, \ldots 8 ; a, b, \ldots=5,6,7,8 ; a_{3}, b_{3}, \ldots=(5,6) ; a_{4}, b_{4}, . .=(7,8) \text {; } \\
& \text { on } T^{*} \mathbf{V} \text { and } T_{s}^{*} \mathbf{V} \text { : } \\
& { }^{\prime} u=\left(x, \quad{ }^{\prime} p\right)=\left\{u^{\alpha}=\left(u^{k}=x^{k}, \quad{ }^{\prime} p_{a}=p_{a}\right)\right\} \\
& =\left({ }_{3}^{1} x,{ }_{4}^{1} p\right)=\left\{{ }^{\prime} u^{\alpha}=\left({ }^{\prime} u^{k_{3}}={ }^{\prime} x^{k_{3}},{ }^{\prime} p_{a_{4}}=p_{a_{4}}\right)\right\}= \\
& { }_{s}^{1} u=\left({ }_{s} x,{ }_{s}^{\prime} p\right)=\left\{{ }^{\prime} u^{\alpha_{s}}=\left(x^{k_{s}}, \quad{ }^{\prime} p_{a_{s}}=p_{a_{s}}\right)\right\}=\left(x^{i_{1}}, x^{i_{2}},{ }^{\prime} p_{a_{3}}=p_{a_{3}}, \quad{ }^{\prime} p_{a_{4}}=p_{a_{4}}\right), \\
& =\left({ }_{3}^{1} u={ }_{3}^{1} x,{ }_{4}^{1} p\right)=\left\{{ }^{\prime} u^{\alpha_{3}}=\left(x^{i_{1}}, x^{i_{2}}, \quad{ }^{\prime} x^{i_{3}} \rightarrow{ }^{\prime} p_{a_{3}}\right), \quad{ }^{\prime} p_{a_{4}}\right\} \text {, } \\
& \text { where }{ }^{\prime} x^{\alpha_{3}}=\left(x^{i_{1}}, x^{a_{2}},{ }^{\prime} p_{a_{3}}=p_{a_{3}}\right) \text {. }
\end{aligned}
$$

$$
\begin{aligned}
& \text { on } T_{\|}^{*} \mathbf{V} \text { and } T_{\| S}^{*} \mathbf{V} \text { : } \\
& \text { " } u=(x, " p)=\left\{" u^{\alpha}=\left(u^{k}=x^{k}, " p_{a}=(i \hbar)^{-1} p_{a}\right)\right\} \\
& =\left({ }_{3}^{11} x,{ }_{4}^{11} p\right)=\left\{\text { " } u^{\alpha}=\left(" u^{k_{3}}={ }^{"} x^{k_{3}}, " p_{a_{4}}=(i \hbar)^{-1} p_{a_{4}}\right)\right\}= \\
& { }_{s}{ }_{s} u=\left({ }_{s} x,{ }_{s}{ }_{s} p\right)=\left\{" u^{\alpha_{s}}=\left(x^{k_{s}}, \quad " p_{a_{s}}=(i \hbar)^{-1} p_{a_{s}}\right)\right\}=\left(x^{i_{1}}, x^{i_{2}}, " p_{a_{3}}=(i \hbar)^{-1} p_{a_{3}}, " p_{a_{4}}=(i \hbar)^{-1} p_{a_{4}}\right) \text {, } \\
& =\left({ }_{3}^{\prime \prime} u={ }_{3}^{11} x,{ }_{4}^{\prime \prime} p\right)=\left\{" u^{\alpha_{3}}=\left(x^{i_{1}}, x^{i_{2}}, " x^{i_{3}} \rightarrow "{ }^{\prime \prime} p_{a_{3}}\right), " p_{a_{4}}\right\} \text {, } \\
& \text { where " } x^{\alpha_{3}}=\left(x^{i_{1}}, x^{i_{2}}, \quad " p_{a_{3}}=(i \hbar)^{-1} p_{a_{3}}\right) \text {. }
\end{aligned}
$$

Such labels of local coordinates and respective splitting via oriented shell by shell indices with $s=1,2,3,4$ can be considered on total spaces of respective (co) tangent bundles and for a phase space model $T_{\|}^{*} \mathbf{V}$ with complex cofibers associated to a real cotangent bundle $T^{*} \mathbf{V}$. The notations from our works are different 
from those introduced for formula (3.1) in [1], where $X_{K}=\left(\frac{p_{k}}{i \hbar}, x^{k}\right)$ and $\hbar=h / 2 \pi$ is the Planck constant, is written instead of $" u^{\alpha}$. Similar coordinates with momentum like variables were used in [2] but with a corresponding adapting for definition of quasi-Hopf structures. In this work and [3], an up (or low, on convenience), label " " " is used for distinguishing coordinates with "complexified momenta" from real phase coordinates ' $u^{\alpha}=\left(x^{k}, p_{a}\right)$ on $T^{*} \mathbf{V}$ (similar conventions are considered in Finsler-Lagrange-Hamilton geometry [4, 5]). Such dyadic coordinates mix under general coordinate transforms. Nevertheless, we can keep a shell oriented labelling if we work with corresponding classes of local (co) frame structure with corresponding adapting to nonholonomic distributions with dyadic decomposition as we explain in next subsection. Dyadic structures, i.e. nonholonomic shell decompositions, s-decompositions and/or s-structures, are important for decoupling with respect to adapted frames various types of modified Einstein equations. The formalism of Nand s-adapted labels and respective abstract or frame coefficient notations used in this and partner works is elaborated in such a way that allows to perform an unified "symbolic" nonholonomic geometric calculus and provide proofs by analogy for various types of nonassociative / noncommutative / commutative spaces with a respective decoupling of physically important systems of PDEs.

\subsubsection{Nonlinear connections and dyadic splitting}

\section{Nonlinear connection structures on commutative spacetimes and phase spaces:}

To study R-flux nonassociative star deformations the phase spaces are modelled as cotangent bundles $T^{*} V$ and $T_{\|}^{*} V$. We shall elaborate on equivalent global and local (co) vector bundles constructions keeping in mind that the complex unity $i$ before momentum like coordinates is used for definition of star products. For simplicity, we shall work with " $u$ coordinates and geometric objects on $T_{\|}^{*} V$ (if necessary, all formulas can be written by analogy without complex unity). The conventional horizontal, h, on base spacetime, and covertical, c, splitting of geometric objects can be defined in global abstract forms by respective Whitney sums $\oplus$ of respective $h$ - and $c$-distributions ( $v$-distributions are used for $T V$ ), when

$$
{ }^{\prime} \mathbf{N}: T \mathbf{T}^{*} \mathbf{V}=h T^{*} V \oplus c T^{*} V \text { and } \quad \text { "N : } T \mathbf{T}_{\|}^{*} \mathbf{V}=h T_{\|}^{*} V \oplus c T_{\|}^{*} V .
$$

For a Lorentz base manifold, $\mathbf{V}$, such a $\mathrm{N}$-connection splitting is with a nonholonomic $4+4$ decomposition which in local forms is written, for instance, "N $\mathbf{N}={ }^{\prime} N_{i a} \frac{\partial}{\partial " p_{a}} \otimes d x^{i}$, using N-connection coefficients " $\mathbf{N}=$ $\left\{" N_{i a}\right\}$. Here we note that in our works spacetime and phase spaces and respective geometric objects are labeled by "bold face" symbols if they are enabled with/ adapted to a N-connection structures and written in so-called $\mathrm{N}$-adapted form. We can always split a Lorentz manifold into conventional h- and v-distributions considering a splitting $\mathbf{N}: T \mathbf{V}=h V \oplus v V$ using corresponding local dyads ${ }^{2} \mathbf{N}=N_{i_{1}}^{i_{2}} \frac{\partial}{\partial x^{i_{2}}} \otimes d x^{i_{1}}$. This is a nonholonomic $(2+2)$-decomposition which is necessary for constructing generic off-diagonal solutions on base manifolds.

\section{N-adapted commutative coframe $4+4$ structures:}

If a N-connection structure is introduced for a real Lorentz spacetime, we can define respective classes of N-elongated (equivalently, N-adapted) bases and dual bases (cobases) for nonholonomic dyadic splitting. Such $s=1$ and 2 dyads are linear on N-connection coefficients,

$$
\begin{aligned}
\mathbf{e}_{i} & =\left(\mathbf{e}_{i_{1}}=\frac{\partial}{\partial x^{i_{1}}}-N_{i_{1}}^{i_{2}}\left(x^{i}\right) \frac{\partial}{\partial x^{i_{2}}}, e_{j_{2}}=\partial_{j_{2}}=\frac{\partial}{\partial x^{j_{2}}}\right), \text { on } T \mathbf{V} ; \\
\mathbf{e}^{i} & =\left(e^{i_{1}}=d x^{i_{1}}, \mathbf{e}^{i_{2}}=d x^{i_{2}}+N_{i_{1}}^{i_{2}}\left(x^{k_{1}}, x^{k_{2}}\right) d x^{i_{1}}\right), \text { on } T^{*} \mathbf{V} .
\end{aligned}
$$

Such a local basis $\mathbf{e}_{k}$ (3) is nonholonomic if the commutators

$$
\mathbf{e}_{[k} \mathbf{e}_{j]}:=\mathbf{e}_{k} \mathbf{e}_{j}-\mathbf{e}_{j} \mathbf{e}_{k}=w_{k j}^{l}(u) \mathbf{e}_{l}
$$


contain nontrivial anholonomy coefficients $w_{k j}^{l}=\left\{w_{i_{1} j_{2}}^{k_{2}}=\partial_{j_{2}} N_{i_{1}}^{k_{2}}, w_{j_{1} i_{1}}^{k_{2}}=\mathbf{e}_{j_{1}} N_{i_{1}}^{k_{2}}-\mathbf{e}_{i_{1}} N_{j_{1}}^{k_{2}}\right\}$. We say that a basis is holonomic (integrable) (when it can be transformed via coordinate transforms into a local coordinated basis, $\left.\mathbf{e}_{k} \rightarrow \partial_{k}=\partial / \partial x^{k}\right)$ if $w_{k j}^{l}=0$.

Similarly, we can define N-elongated dual bases and cobases for cotangent bundles, for instance, on a (complex) phase space,

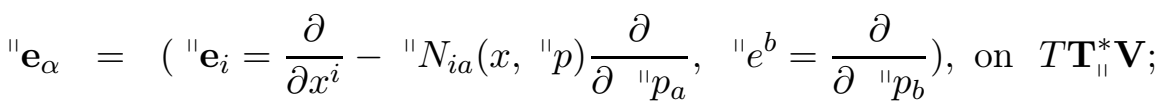

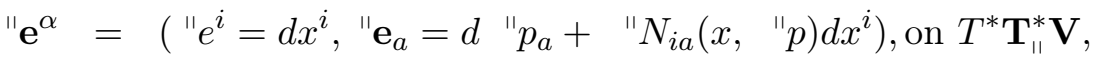

where $\partial_{i}:=\partial / \partial x^{i}, \quad " \partial^{b}:=\partial / \partial " p_{b}, \partial_{\alpha}:=\left(\partial_{i}, \partial_{a}\right), \quad " \partial_{\alpha}:=\left(\partial_{i}, \quad " \partial^{a}\right)$ etc.

\section{Nonholonomic dyadic decomposition (s-decomposition):}

Total spaces' respective $(2+2)+(2+2)$ splitting, see similar constructions in [4, 5, 20, 21, 23, can be defined in global forms by respective classes of $\mathrm{N}$-connection structures:

$$
\begin{aligned}
& { }_{s} \mathbf{N}:{ }_{s} T \mathbf{T}^{*} \mathbf{V}={ }^{1} h T^{*} V \oplus{ }^{2} v T^{*} V \oplus{ }^{3} c T^{*} V \oplus{ }^{4} c T^{*} V \text { and } \\
& { }_{s}{ }_{s} \mathbf{N}: \\
& { }_{s} T \mathbf{T}_{\|}^{*} \mathbf{V}={ }^{1} h T_{\|}^{*} V \oplus{ }^{2} v T_{\|}^{*} V \oplus{ }^{3} c T_{\|}^{*} V \oplus{ }^{4} c T_{\|}^{*} V,
\end{aligned}
$$

when respective decompositions into conventional 2-dim nonholonomic distributions of $T T V, T T^{*} V$ and $T T_{\|}^{*} V$,

$$
\begin{aligned}
& \operatorname{dim}\left({ }^{1} h T^{*} V\right)=\operatorname{dim}\left({ }^{2} v T^{*} V\right)=\operatorname{dim}\left({ }^{3} c T^{*} V\right)=\left({ }^{4} c T^{*} V\right)=2 \text { and } \\
& \operatorname{dim}\left({ }^{1} h T_{\|}^{*} V\right)=\operatorname{dim}\left({ }^{2} v T_{\|}^{*} V\right)=\operatorname{dim}\left({ }^{3} c T_{\|}^{*} V\right)=\left({ }^{4} c T_{\|}^{*} V\right)=2 .
\end{aligned}
$$

In these formulas, left up labels like ${ }^{1} h,{ }^{3} c$ etc. state that using nonholonomic (equivalently, anholonomic and/or non-integrable) distributions we split respective 8-d total spaces into oriented 2-d shells with numbers $1,2,3$ and 4 .

The nonholonomic dyadic splitting with N-connections (6) are defined locally, for instance, by such coefficients

$$
\begin{aligned}
{ }_{s} \mathrm{~N}= & \left\{N_{i_{1}}^{i_{2}}\left(x^{i_{1}}, x^{a_{2}}\right), "{ }^{\prime} N_{i_{1} a_{3}}\left(x^{i_{1}}, x^{a_{2}}, " p_{b_{3}}\right), "{ }^{\prime} N_{i_{1} a_{4}}\left(x^{i_{1}}, x^{a_{2}}, " p_{b_{3}}, " p_{b_{4}}\right),\right. \\
& \left." N_{i_{2} a_{3}}\left(x^{i_{1}}, x^{a_{2}}, " p_{b_{3}}\right),{ }^{\prime \prime} N_{i_{2} a_{4}}\left(x^{i_{1}}, x^{a_{2}}, " p_{b_{3}}, " p_{b_{4}}\right),{ }^{\text {" }} N_{a_{4}}^{a_{3}}\left(x^{i_{1}}, x^{a_{2}}, " p_{b_{3}}, " p_{b_{4}}\right)\right\} .
\end{aligned}
$$

Such values with "shell by shell" decompositions allow us to define N-adapted bases (s-adapted, or equivalently, s-bases),

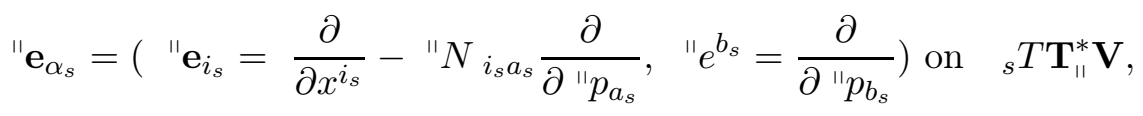

$$
\begin{aligned}
& \text { when } \quad \mathbf{e}_{\alpha_{1}}=\left(" e_{i_{1}}=\frac{\partial}{\partial x^{i_{1}}}=\partial_{i_{1}}\right) \text {, for } i_{1}=1,2 \text {; }
\end{aligned}
$$

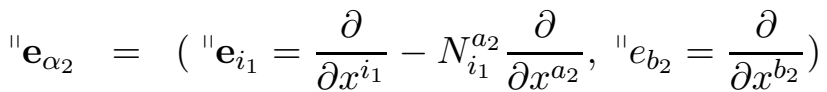

$$
\begin{aligned}
& =\left({ }^{~ "} \mathbf{e}_{i_{1}}=\partial_{i_{1}}-N_{i_{1}}^{a_{2}} \partial_{a_{2}}, \quad \text { " } e_{b_{2}}=\partial_{b_{2}}\right) \text {, for } b_{2}=3,4 \\
& " \mathbf{e}_{\alpha_{3}}=\left(" \mathbf{e}_{i_{2}}=\frac{\partial}{\partial x^{i_{2}}}-"{ }^{\prime} N_{i_{2} a_{3}} \frac{\partial}{\partial " p_{a_{3}}}, " e^{b_{3}}=\frac{\partial}{\partial " p_{b_{3}}}\right), \\
& =\left({ }^{"} \mathbf{e}_{i_{2}}=\partial_{i_{2}}-{ }^{"} N_{i_{2} a_{3}}{ }^{"} \partial^{a_{3}}, " e^{b_{3}}="{ }^{b_{3}}\right) \text {, for } i_{2}=\left(i_{1}, a_{2}\right) \text {; } \\
& " \mathbf{e}_{\alpha_{4}}=\left(" \mathbf{e}_{i_{3}}=\frac{\partial}{\partial x^{i_{3}}}-" N_{i_{3} a_{4}} \frac{\partial}{\partial " p_{a_{4}}}, " e^{b_{4}}=\frac{\partial}{\partial " p_{b_{4}}}\right) \\
& =\left({ }^{\prime} \mathbf{e}_{i_{3}}=\partial_{i_{3}}-{ }^{"} N_{i_{3} a_{4}} " \partial^{a_{4}}, \quad " e^{b_{4}}=" \partial^{b_{4}}\right) \text {, for } i_{3}=\left(i_{1}, a_{2}, a_{3}\right) \text {, }
\end{aligned}
$$


where we follow the conventions for dyadic indices and coordinates (11).

For dual dyadic shell by shell s-adapted bases to (8), s-cobases, we have

$$
\begin{aligned}
& \text { "e }{ }^{\alpha_{s}}=\left("{ }^{i_{s}}=d x^{i_{s}}, " \mathbf{e}_{a_{s}}=d " p_{a_{s}}+{ }^{"} N_{i_{s} a_{s}} d x^{i_{s}}\right) \text { on }{ }_{s} T^{*} \mathbf{T}_{\|}^{*} \mathbf{V} \text {, when } \\
& \text { " } \mathbf{e}^{\alpha_{4}}=\left({ }^{~ " e^{i_{3}}}=d x^{i_{3}}, " \mathbf{e}_{a_{4}}=d " p_{a_{4}}+{ }^{"} N_{i_{3} a_{4}} d x^{i_{3}}\right), \\
& " \mathbf{e}^{\alpha_{3}}=\left(\mathbf{e}^{i_{2}}=d x^{i_{2}}, " \mathbf{e}_{a_{3}}=d " p_{a_{3}}+"{ }^{\prime \prime} N_{i_{2} a_{3}} d x^{i_{2}}\right), \\
& \text { "e } \mathbf{e}^{\alpha_{2}}=\left(\mathbf{e}^{i_{1}}=d x^{i_{1}}, " \mathbf{e}_{a_{2}}=d " p_{a_{3}}+{ }^{\prime \prime} N_{i_{2} a_{3}} d x^{i_{2}}\right), \\
& \text { "e } \mathbf{e}^{\alpha_{1}}=\left(\mathbf{e}^{i_{1}}=d x^{i_{1}}\right)
\end{aligned}
$$

defined by the same N-connection coefficients (7) for respective s-decomposition (in brief, we shall write also s-connection defining corresponding nonholonomic s-structures) phase space.

\subsection{Nonassociativity, dyadic N-connections, and quasi-Hopf algebras}

We generalize for phase spaces with dyadic decomposition the concept of nonassociative star product considered in [39, 38]. The nonassociative geometric constructions were re-defined on nonholonomic phase spaces and spacetime manifolds enabled with N-connection structure in our partner work [3]). The formulas provided is section 2 of [1] and section 2 of [2] for configuration spaces can be obtained in the cases of trivial N-connection s-structures.

\subsubsection{Nonassociative star product and dyadic s-structures}

We begin with the definition of nonassociative star products in h-coordinates for h-coefficients with decompositions in shells $s=1,2$ in order to understand the geometric principles for dyadic and nonholonomic modifications of coordinate frame constructions in [1, 39, 38]. In next subsections, the formalism of nonassociative star product is developed in s-adapted form on the full phase space $T_{\| S}^{*} \mathbf{V}$, involving four shell by shell decompositions. The constructions will be extended to the case of nontrivial quasi-Hopf structures with s-splitting in order to formulate a corresponding self-consistent geometric approach with star deformations of coordinate diffeomorphism in s-adapted forms.

Let us consider a full phase space $\mathcal{M}$ containing a spacetime direction determined by a $\mathrm{N}$-adapted direction

$' \mathbf{e}_{i_{s}}$ and a momentum like cofiber directions ${ }^{\prime} e^{b_{s}}$. For any two functions $z(x, p)$ and $q(x, p)$, we define a sadapted star product $\star_{s}$ :

$$
\begin{aligned}
z \star_{s} q & :=\cdot\left[\mathcal{F}_{s}^{-1}(z, q)\right] \\
& =\cdot\left[\exp \left(-\frac{1}{2} i \hbar\left(\mathbf{e}_{i_{s}} \otimes{ }^{\prime} e^{i_{s}}-{ }^{\prime} e^{i_{s}} \otimes \mathbf{e}_{i_{s}}\right)\right)+\frac{i \ell_{s}^{4}}{12 \hbar} R^{i_{s} j_{s} a_{s}}\left(p_{a_{s}}{ }^{\prime} \mathbf{e}_{i_{s}} \otimes \mathbf{e}_{j_{a}}-{ }^{\prime} \mathbf{e}_{j_{s}} \otimes p_{a_{s}}{ }^{\prime} \mathbf{e}_{i_{s}}\right)\right] z \otimes q \\
& =z \cdot q-\frac{i}{2} \hbar\left[\left({ }^{\prime} \mathbf{e}_{i_{s}} z\right)\left({ }^{\prime} e^{i_{s}} q\right)-\left({ }^{\prime} e^{i_{s}} z\right)\left({ }^{\prime} \mathbf{e}_{i_{s}} q\right)\right]+\frac{i \ell_{s}^{4}}{6 \hbar} R^{i_{s} j_{s} a_{s}} p_{a_{s}}\left({ }^{\prime} \mathbf{e}_{i_{s}} z\right)\left({ }^{\prime} \mathbf{e}_{j_{s}} q\right)+\ldots
\end{aligned}
$$

where the constant $\ell$ defines R-flux contributions for a antisymmetric $R^{i_{s} j_{s} a_{s}}$ background in string theory, with s-indices. In these formulas, the tensor product $\otimes$ is used in a form indicating on which factor of $z \otimes q$ the Nadapted derivatives act with the dot form, when eventually the tensor products turn into usual multiplications. In coordinate frames and for holonomic frames determined by trivial N-connection structures, the star product (10) coincides with that considered in [1]. In [3], we write $\star_{N}$ and extend the concept of nonholonomic star product on total phase space (see next subsection for constructions with quasi-Hopf structure). In coordinate base cases, we do not write boldface symbols and do not use the labels "s" or "N" for star products and related geometric objects. 


\subsubsection{Quasi-Hopf s-structures}

We consider the universal enveloping Hopf algebra $U \operatorname{Vec}(\mathcal{M})$ of the Lie algebra of vector fields $\operatorname{Vec}(\mathcal{M})$ on a nonholonomic manifold (it can be with s-structure) $\mathcal{M}$, see such constructions for holonomic structures in [2]. For non-trivial N-connection s-structures of phase space $\mathcal{M}=T_{\|}^{*} \mathbf{V}$, we elaborate on a nonholonomic algebraic and dyadic formalism with d-vectors and d-tensors. A nonholonomic splitting $\operatorname{Vec}(\mathcal{M})=h \operatorname{Vec}(\mathcal{M}) \oplus v \operatorname{Vec}(\mathcal{M})$ follows from the definition of N-connection structure (21). This defines a Hopf d-algebra $U \operatorname{Vec}\left(\mathcal{M},{ }_{s} N\right)$. A $\mathrm{N}$-adapted cochain twist element $\mathcal{F}_{N}$ with possible s-decomposition $\mathcal{F}_{s}$ is parameterized

$$
\begin{aligned}
& \text { " } \mathcal{F}_{N}={ }^{\prime} \check{\mathcal{F}}_{N}{ }^{\prime} \check{\mathcal{F}}_{R N}={ }^{\prime} \check{\mathcal{F}}_{R N}{ }^{\prime} \check{\mathcal{F}}_{N}= \\
& " \mathcal{F}_{s}=" \check{\mathcal{F}}_{s} \mid \check{\mathcal{F}}_{R s}=1 \check{\mathcal{F}}_{R s} " \check{\mathcal{F}}_{s}
\end{aligned}
$$

for a nonholonomic Hopf 2-cocycle (determining a nonholonomic Moyal-Weyl deformation of the phase space)

$$
\text { " } \check{\mathcal{F}}_{N}=\exp \left[\left(" \mathbf{e}_{i} \otimes " e^{n+i}-" e^{n+i} \otimes " \mathbf{e}_{i}\right)\right]=" \check{\mathcal{F}}_{s}=\exp \left[\left(" \mathbf{e}_{i_{s}} \otimes " e^{n+i_{s}}-" e^{n+i_{s}} \otimes " \mathbf{e}_{i_{s}}\right)\right],
$$

where for 4 -d base Lorentz manifolds $i=\left(i_{1}, i_{2}\right)$, or $i=\left(i_{1}, a_{2}\right)$, (with $i_{1}=1,2, a_{2}=3$,4); such indices are used for contacting in oriented form shall splitting of indices $a=\left(a_{3}=n+i_{1}, a_{4}=n+i_{2}\right)$ for a h1-v2 splitting of coefficients of geometric s-objects. Respective nonholonomic 2-cocycles for a R-flux are

$$
\begin{aligned}
& \check{\mathcal{F}}_{R N}=\exp \left[\frac{i \kappa}{2} R^{j k a}\left(p_{a} \mathbf{e}_{k} \otimes{ }^{\prime} \mathbf{e}_{j}-{ }^{\prime} \mathbf{e}_{j} \otimes p_{a}{ }^{\prime} \mathbf{e}_{k}\right)\right]= \\
& \check{\mathcal{F}}_{R s}=\exp \left[\frac{i \kappa}{2} R^{j_{s} k_{s} a_{s}}\left(p_{a_{s}}{ }^{\prime} \mathbf{e}_{k_{s}} \otimes{ }^{\prime} \mathbf{e}_{j_{s}}-{ }^{\prime} \mathbf{e}_{j_{s}} \otimes p_{a_{s}}{ }^{\prime} \mathbf{e}_{k_{s}}\right)\right],
\end{aligned}
$$

where $\kappa:=\ell_{s}^{3} / 6 \hbar$ and $\hbar$ are treated as independent small deformation parameters (in some formulas, we shall us as parameters the values $\ell_{s}$ and $\left.\hbar\right)$. The twist d-operators can be parameterized in terms of respective elements with dyadic splitting of indices $\mathfrak{f}^{\alpha_{s}}, \mathfrak{f}_{\beta_{s}} \in U \operatorname{Vec}(\mathcal{M})$ when

$$
\begin{aligned}
" \check{\mathcal{F}}_{N}:=\mathfrak{f}^{\alpha} \otimes \mathfrak{f}_{\alpha}=" \check{\mathcal{F}}_{s}=\mathfrak{f}^{\alpha_{s}} \otimes \mathfrak{f}_{\alpha_{s}}=1 \otimes 1+O\left(\hbar, \ell_{s}^{3}\right), \\
\text { with inverse twist } " \check{\mathcal{F}}_{N}^{-1}:=\overline{\mathfrak{f}}^{\alpha} \otimes \overline{\mathfrak{f}}_{\alpha}=" \check{\mathcal{F}}_{s}^{-1}:=\overline{\mathfrak{f}}^{\alpha_{s}} \otimes \overline{\mathfrak{f}}_{\alpha_{s}} ; \\
" \check{\mathcal{F}}_{R N}:=\mathfrak{f}_{R N}^{\alpha} \otimes \mathfrak{f}_{\alpha}^{R N}=" \check{\mathcal{F}}_{R s}:=\mathfrak{f}_{R s}^{\alpha_{s}} \otimes \mathfrak{f}_{\alpha_{s}}^{R s}=1 \otimes 1+O(\kappa),
\end{aligned}
$$

where summation on low-up repeating indices and possible shall by shall splitting is understood.

A Hopf s-algebra $U \operatorname{Vec}\left(\mathcal{M},{ }_{s}^{\prime \prime} N\right)$ is determined by such N-adapted structures:

a co-product $\boldsymbol{\Lambda}$ defined as $\mathbf{\Lambda}(1)=1 \otimes 1, \mathbf{\Lambda}\left(" \mathbf{e}_{\alpha_{s}}\right)=1 \otimes " \mathbf{e}_{\alpha_{s}}+"{ }^{\prime} \mathbf{e}_{\alpha_{s}} \otimes 1 ;$

a co-unit $\epsilon$ defined as $\epsilon(1)=1, \epsilon\left(" \mathbf{e}_{\alpha_{s}}\right)=0$; and

and an antipode $\mathbf{S}$ defined as $\mathbf{S}(1)=1, \mathbf{S}\left(" \mathbf{e}_{\alpha_{s}}\right)=-{ }^{\prime} \mathbf{e}_{\alpha_{s}}$; such $\mathbf{\Delta}$ and $\epsilon$ d-operators are extended to the total space $U \operatorname{Vec}(\mathcal{M}, N)$ as d-algebra homomorphisms and $\mathbf{S}$ extended as an d-algebra anti-homomorphism (linear, anti-multiplicative and N-adapted). The N-connection s-splitting states a s-decomposition $\boldsymbol{\Lambda}=$ $\left(h \mathbf{\Delta},{ }^{s} v \mathbf{\Delta}\right)$.

A quasi-Hopf s-algebra $U \operatorname{Vec}^{\mathcal{F}}\left(\mathcal{M},{ }_{s}^{\|} N\right)$ is generated as an extension in s-adapted form with power series in $\hbar$ and $\kappa$ of a Hopf d-algebra $U \operatorname{Vec}\left(\mathcal{M},{ }_{s} N\right)$ using a twist " $\mathcal{F}_{s}$ (11). The algebraic Hopf s-structure is preserved for respective $\mathcal{F}$-extended s-objects: co-product $\boldsymbol{\Lambda}_{\mathcal{F}}=\mathcal{F}_{N} \boldsymbol{\Lambda}_{N}^{-1}=\boldsymbol{\Lambda}_{\mathcal{F}}=\mathcal{F}_{s} \boldsymbol{\Lambda} \mathcal{F}_{s}^{-1}$; quasi-antipode $\mathbf{S}_{\mathcal{F}}=\mathbf{S}$; and co-unit $\epsilon_{\mathcal{F}}=\epsilon$. Such s-operators satisfy the properties:

$$
\begin{aligned}
& \mathbf{\Delta}_{\mathcal{F}}\left(" \mathbf{e}_{i_{s}}\right)=1 \otimes " \mathbf{e}_{i_{s}}+"{ }^{\prime} \mathbf{e}_{i_{s}} \otimes 1, \mathbf{\Delta}_{\mathcal{F}}\left(" e^{a_{s}}\right)=1 \otimes " e^{a_{s}}+" e^{a_{s}} \otimes 1+i \kappa R^{j_{s} k_{s} a_{s}} " \mathbf{e}_{j_{s}} \otimes " \mathbf{e}_{k_{s}} \text { and } \\
& \mathfrak{f}^{\alpha} \mathbf{S}\left(\mathfrak{f}_{\alpha}\right)=\mathfrak{f}_{R N}^{\alpha} \mathbf{S}\left(\mathfrak{f}_{\alpha}^{R N}\right)=\mathfrak{f}^{\alpha_{s}} \mathbf{S}\left(\mathfrak{f}_{\alpha_{s}}\right)=\mathfrak{f}_{R s}^{\alpha_{s}} \mathbf{S}\left(\mathfrak{f}_{\alpha_{s}}^{R s}\right)= \\
& \overline{\mathfrak{f}}^{\alpha} \mathbf{S}\left(\overline{\mathfrak{f}}_{\alpha}\right)=\overline{\mathfrak{f}}_{R N}^{\alpha} \mathbf{S}\left(\overline{\mathfrak{f}}_{\alpha}^{R N}\right)=\overline{\mathfrak{f}}^{\alpha_{s}} \mathbf{S}\left(\overline{\mathfrak{f}}_{\alpha_{s}}\right)=\overline{\mathfrak{f}}_{R s}^{\alpha_{s}} \mathbf{S}\left(\overline{\mathfrak{f}}_{\alpha_{s}}^{R s}\right)=1 .
\end{aligned}
$$


In holonomic and N- or s-adapted variants twist d-operators " $\mathcal{F}_{N}$ or " $\mathcal{F}_{s}$ do not fulfill the 2-cocycle condition. In such cases, one considers respective associator $\mathrm{N}$ - and s-operators. For Hopf d-algebraic structures, we use respective associator operations $\Phi$ (defining a Hopf 3-cocycle; and its inverse associator $\Phi^{-1}$,

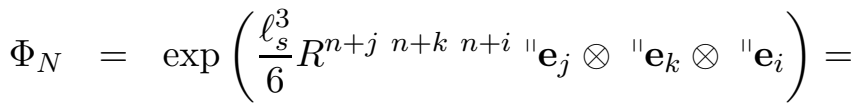

$$
\begin{aligned}
& \Phi_{s}=\exp \left(\frac{\ell_{s}^{3}}{6} R^{n+j_{s} n+k_{s} n+i_{s}} " \mathbf{e}_{j_{s}} \otimes " \mathbf{e}_{k_{s}} \otimes " \mathbf{e}_{i_{s}}\right)={ }_{1} \phi \otimes{ }_{2} \phi \otimes{ }_{3} \phi=1 \otimes 1 \otimes 1+O\left(\ell_{s}^{3}\right), \\
& \text { and } \Phi_{N}^{-1}=\Phi_{s}^{-1}={ }_{1} \bar{\phi} \otimes{ }_{2} \bar{\phi} \otimes{ }_{3} \bar{\phi} \text {. }
\end{aligned}
$$

For instance, for s-operators, the co-product $\boldsymbol{\Lambda}_{\mathcal{F}}$ is not co-associative because the 2-cocycle condition is not satisfied and the property

$$
\Phi_{s}\left(" \mathcal{F}_{s} \otimes 1\right)(\mathbf{\Delta} i d \otimes 1) " \mathcal{F}_{s}=\left(1 \otimes " \mathcal{F}_{s}\right)(\mathbf{\Delta} i d \otimes 1) " \mathcal{F}_{s}
$$

results in a quasi-associativity condition

$$
\Phi_{s}\left(\boldsymbol{\Delta}_{\mathcal{F}} i d \otimes 1\right) \boldsymbol{\Lambda}_{\mathcal{F}}(\xi)=\left(i d \otimes \boldsymbol{\Delta}_{\mathcal{F}}\right) \boldsymbol{\Delta}_{\mathcal{F}}(\xi) \Phi_{s}, \forall \xi \in U \operatorname{Vec}\left(\mathcal{M},{ }_{s}{ }_{s} N\right)
$$

Considering dyadic decompositions, we use a sextuple $\left(U \operatorname{Vec}\left(\mathcal{M},{ }_{s} N\right), \bullet, \boldsymbol{\Lambda}_{\mathcal{F}}, \Phi_{s}, \mathbf{S}, \epsilon\right)$ and define on the dvector space $U \operatorname{Vec}\left(\mathcal{M},{ }_{s} N\right)$, i.e. on ${ }_{s} T \mathcal{M}=T T_{\| s}^{*} \mathbf{V}$, a structure of quasi-Hopf s-algebra $U \operatorname{Vec}^{\mathcal{F}}\left(\mathcal{M},{ }_{s} N\right)$. For our constructions, there are we considered boldface d-operators in order to emphasize that we work with s-adapted algebraic and geometric structures. We substitute " $\mathbf{e}_{\alpha_{s}} \rightarrow " \partial_{\alpha_{s}}$ and changing respectively $\mathbf{\Delta}_{\mathcal{F}} \rightarrow \triangle_{\mathcal{F}}, \Phi_{s} \rightarrow \Phi, \mathbf{S} \rightarrow S$ etc., we obtain the definition of standard Hopf algebra $U \operatorname{Vec}(\mathcal{M})$ and coordinate base constructions as in [37, 2, 38. For N-connection splitting, such d-algebras were introduced in [3].

In nonholonomic dyadic form, we can formulate such principles of s-adapted twist deformation: We write $\mathcal{A}_{s}=\left(h \mathcal{A},{ }^{s} v \mathcal{A}\right)$ for a d-algebra with s-adapted representation of a Hopf d-algebra $U \operatorname{Vec}\left(\mathcal{M},{ }_{s} N\right)$ and a twist deformation induced by a R-flux into a quasi-Hopf d-algebra $U \operatorname{Vec}^{\mathcal{F}}\left(\mathcal{M},{ }_{s} N\right)$. Any d-vector $\vartheta=\left(h \vartheta,{ }^{s} v \vartheta\right) \in$ $\operatorname{Vec}\left(\mathcal{M},{ }_{s} N\right)$ acts on $\mathcal{A}_{s}$. This action is performed following such steps:

1) For every algebraic product $a b$, we consider $\vartheta(a b)=u(a) b+a u(b)$ and we have a $U \operatorname{Vec}\left(\mathcal{M},{ }_{s}^{\prime \prime} N\right)$-module d-algebra $\mathcal{A}_{s}$.

2) This multiplication is deformed into a $\mathrm{N}$ - and s-adapted star multiplication

$$
\begin{aligned}
a \star_{N} b & =\overline{\mathfrak{f}}^{\alpha}(a) \overline{\mathfrak{f}}_{\alpha}(b)=\overline{\mathfrak{f}}^{i}(a) \overline{\mathfrak{f}}_{i}(b)+" \overline{\mathfrak{f}}_{c}(a) " \overline{\mathfrak{f}}^{c}(b)= \\
a \star_{s} b & =\overline{\mathfrak{f}}^{\alpha_{s}}(a) \overline{\mathfrak{f}}_{\alpha_{s}}(b)=\overline{\mathfrak{f}}^{i_{s}}(a) \overline{\mathfrak{f}}_{i_{s}}(b)+" \overline{\mathfrak{f}}_{c_{s}}(a) " \overline{\mathfrak{f}}^{c_{s}}(b)
\end{aligned}
$$

resulting in a noncommmutative and nonassociative s-algebra $\mathcal{A}_{s}^{\star}=\left(h \mathcal{A}^{\star},{ }^{s} v \mathcal{A}^{\star}\right)$.

Any $\mathcal{A}_{N}^{\star}$ and/or carries $\mathcal{A}_{s}^{\star}$ a respective representation of the quasi-Hopf d-algebra $U V e c^{\mathcal{F}}\left(\mathcal{M},{ }_{s} N\right)$ because for any element $\xi$ of this d-algebra we prove in $\mathrm{N}$ - and s-adapted form that

$$
\begin{aligned}
\xi\left(a \star_{N} b\right) & =\xi\left(\overline{\mathfrak{f}}^{\alpha}(a) \overline{\mathfrak{f}}_{\alpha}(b)\right)=\xi_{\left(1_{0}\right)}\left(\overline{\mathfrak{f}}^{\alpha}(a)\right) \xi_{\left(2_{0}\right)}\left(\overline{\mathfrak{f}}_{\alpha}(b)\right)=\overline{\mathfrak{f}}^{\alpha}\left(\xi_{\left(1_{0}\right)}(a)\right) \xi_{\left(2_{0}\right)}\left(\overline{\mathfrak{f}}_{\alpha}(b)\right)= \\
\xi\left(a \star_{s} b\right) & =\xi\left(\overline{\mathfrak{f}}^{\alpha_{s}}(a) \overline{\mathfrak{f}}_{\alpha_{s}}(b)\right)=\xi_{\left(1_{0}\right)}\left(\overline{\mathfrak{f}}^{\alpha_{s}}(a)\right) \xi_{\left(2_{0}\right)}\left(\overline{\mathfrak{f}}_{\alpha_{s}}(b)\right)=\overline{\mathfrak{f}}^{\alpha_{s}}\left(\xi_{\left(1_{0}\right)}(a)\right) \xi_{\left(2_{0}\right)}\left(\overline{\mathfrak{f}}_{\alpha_{s}}(b)\right) .
\end{aligned}
$$

In such formulas, there are used un-deformed N- and s-adapted co-products:

$$
\begin{aligned}
\mathbf{\Delta}(\xi) & =\xi_{\left(1_{0}\right)} \otimes_{N} \xi_{\left(2_{0}\right)} \text { when } \mathbf{\Delta}(\xi) \mathcal{F}_{N}^{-1}=\mathcal{F}_{N}^{-1} \boldsymbol{\Delta}_{\mathcal{F}}(\xi), \text { and } \\
\mathbf{\Lambda}(\xi) & =\xi_{\left(1_{0}\right)} \otimes_{s} \xi_{\left(2_{0}\right)} \text { when } \mathbf{\Delta}(\xi) \mathcal{F}_{s}^{-1}=\mathcal{F}_{s}^{-1} \boldsymbol{\Lambda}_{\mathcal{F}}(\xi) .
\end{aligned}
$$

Such constructions can be used for N- and s-adapting other type of algebraic structures (for instance, Clifford algebras, octonionic algebras etc.). 


\section{Hopf s-algebras, dyadic $\mathbf{N}$-adapted Lie derivatives, and $\mathcal{R}$ action on functions on $T_{\|}^{*} \mathbf{V}$ :}

Hereafter, we provide only the formulas for s-structures. For a commutative d-algebra $\mathcal{A}_{s}$ and it s-adapted star deformation $\mathcal{A}_{s}^{\star}$, we can control noncommutativity via shall by shall action of $\mathcal{R}$-matrix, $\mathcal{R}_{s}=\mathcal{F}_{s}^{-2}$, when for the star product (12) of two elements $a$ and $b$ is computed

$$
\begin{aligned}
a \star_{s} b & =\bar{R}^{\gamma}(b) \star_{s} \bar{R}_{\gamma}(a):=b^{\bar{\gamma}} \star_{s} a \bar{\gamma}=b^{\bar{k}} \star_{s} a_{\bar{k}}+" b_{\bar{c}} \star_{N} \text { " } a^{\bar{c}}, \\
& =\overline{\mathfrak{f}}^{\alpha_{s}}(a) \overline{\mathfrak{f}}_{\alpha_{s}}(b)=\overline{\mathfrak{f}}_{\alpha_{s}}(b) \overline{\mathfrak{f}}^{\alpha_{s}}(a), \text { where } b^{\bar{\gamma}_{s}}:=\bar{R}^{\gamma_{s}}(b) \text { and } a_{\bar{\gamma}_{s}}:=\bar{R}_{\gamma_{s}}(a) .
\end{aligned}
$$

For an associative d-algebra $\mathcal{A}_{s}$ is associative, we can consider the associator d-operator

$$
\Phi:\left(a \star_{s} b\right) \star_{s} c={ }^{\phi_{1}} a \star_{s}\left({ }^{\phi_{2}} b \star_{s}{ }^{\phi_{3}} c\right),
$$

where, for instance, ${ }^{\phi_{1}} a=\phi_{1}(a)$.

To understand how the quasi-Hopf and dyadic structures can be adapted to act in a distinguished selfconsistent form we can consider a simplest case of algebraic s-operations for a function $q \in C^{\infty}\left(\mathcal{M},{ }_{s}^{\prime \prime} N\right)$. This can be for the space of functions is $C^{\infty}(\mathcal{M})$ when the usual partial derivatives can be transformed into N-adapted and/or s-adapted ones. The Lie d-derivative $\mathcal{L}_{\xi}(q):=\xi(q)$ can be defined in s-adapted form when any d-vector field $\xi=\xi^{\beta_{s}}$ " $\mathbf{e}_{\beta_{s}}$ is subjected to a s-adapted dyadic decomposition. The action of the Lie s-algebra $\operatorname{Vec}\left(\mathcal{M},{ }_{s} N\right)$ on such functions can be extended to an action of the universal enveloping d-algebra $U \operatorname{Vec}\left(\mathcal{M},{ }_{s} N\right)$ if we define the Lie d-derivative (we may write equivalently s-derivative, respectively, s-vectors) on products of s-vectors $\mathcal{L}_{{ }_{1}{ }^{2}{ }_{\xi} \ldots m_{\xi}}:=\mathcal{L}_{{ }_{1}} \circ \mathcal{L}_{{ }_{2}} \circ \ldots \circ \mathcal{L}{ }_{m \xi}$ and considering decompositions by linearity.

At the next step, we deform with power series on $\hbar$ and $\kappa$ the $U \operatorname{Vec}\left(\mathcal{M},{ }_{s} N\right)$-module d-algebra $C^{\infty}\left(\mathcal{M},{ }_{s} N\right)$ into $U \operatorname{Vec}^{\mathcal{F}}\left(\mathcal{M},{ }_{s}^{"} N\right)$-module d-algebra $\mathcal{A}_{s}^{\star}:=C_{\star}^{\infty}\left(\mathcal{M},{ }_{s} N\right)$ for which the d-vector space is considered the same as $C^{\infty}\left(\mathcal{M},{ }_{s}^{\prime \prime} N\right)$ but with multiplication defined by a s-adapted star product (10). We state that for two functions $q, z \in C^{\infty}\left(\mathcal{M},{ }_{s}^{\prime \prime} N\right)$, the s-adapted star product possess such dyadic properties:

$$
\begin{aligned}
& q \star_{s} z=\overline{\mathfrak{f}}^{\alpha_{s}}(q) \cdot \overline{\mathfrak{f}}_{\alpha_{s}}(z) \\
& =\bar{R}^{\alpha_{s}}(z) \star_{s} \bar{R}_{\alpha_{s}}(q):=z^{\bar{\gamma}_{s} \star_{s}} q_{\bar{\gamma}_{s}} \text { controls noncommutativity ; } \\
& \text { and } \Phi: \quad\left(z \star_{s} q\right) \star_{s} f={ }^{\phi_{1}} z \star_{s}\left({ }^{\phi_{2}} q \star_{s}{ }^{\phi_{3}} f\right) \text { controls nonassociativity. }
\end{aligned}
$$

In these formulas, the constant function 1 on $\mathcal{M}$ is also the unit of the star s-algebra $\mathcal{A}_{s}^{\star}$ which is stated by the property that $q \star_{s} 1=q=1 \star_{s} q$.

We can consider a s-adapted star commutator of functions, $[q, z]_{\star s}=q \star_{s} z-z \star_{s} q$, and define on nonholonomic dyadic decompositions on phase space $\left(\mathcal{M},{ }_{s}^{\prime \prime} N\right)$ a quasi-Poisson coordinate s-algebra:

$$
\left[x^{j_{s}}, x^{k_{s}}\right]_{\star s}=2 i \kappa R^{n+j_{s} n+k_{s} a_{s}} p_{a_{s}},\left[x^{i_{s}}, p_{n+j_{s}}\right]_{\star s}=i \hbar \delta_{j_{s}}^{i_{s}} \text { and }\left[p_{n+i_{s}}, p_{n+j_{s}}\right]_{\star s}=0,
$$

for a parabolic R-flux nonholonomic background, and a nontrivial Jacobiator

$$
\left[x^{i_{s}}, x^{j_{s}}, x^{k_{s}}\right]_{\star_{s}}=\ell_{s}^{3} R^{n+i_{s}} n+j_{s} n+k_{s} .
$$

We note that such formal coordinate functions on $T_{\| s}^{*} \mathbf{V}$ are computed with indices determined with respect to $" \mathbf{e}_{\beta_{s}}$ (죠) and following the conventions on s-coordinates (1).

\subsection{Forms and d-tensors for quasi-Hopf s-adapted structures}

\subsubsection{Nonassociative dyadic deformations of star exterior products}

We denote the exterior algebra of differential forms with dyadic decomposition $\Omega^{\natural}\left(\mathcal{M},{ }_{s}^{\prime \prime} N\right)$ and study Nadapted nonassociative nonholonomic s-deformations of star exterior products, which is written $\Omega_{\star}^{\natural}\left(\mathcal{M},{ }_{s}^{\|} N\right)$. 
The constructions start with zero-forms (which are functions with star s-adapted quasi-Hopf product (14), $\Omega_{\star}^{0}\left(\mathcal{M},{ }_{s} N\right)=\mathcal{A}_{s}^{\star}$. Then, the algebra of differential forms is defined in a standard nonholonomic form but with nonassociative product $\wedge_{\star_{s}}$. We state that for any couple of 1 -forms $" \omega=\left\{{ }_{s}{ }_{s} \omega\right\}, "{ }^{\prime \prime} \gamma=\left\{{ }_{s}{ }^{\prime \prime} \gamma\right\} \in \Omega_{\star}^{1}\left(\mathcal{M},{ }_{s} N\right)$

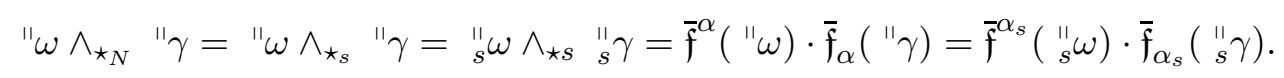

The exterior derivative can be written in s-adapted form,

$$
\text { "d } \mathbf{d}=\left\{{ }_{\mathrm{s}}^{\|} \mathbf{d}\right\}: \Omega_{\star}^{\natural}\left(\mathcal{M},{ }_{s}^{\|} N\right) \rightarrow \Omega_{\star}^{\natural+1}\left(\mathcal{M},{ }_{s}^{\|} N\right) .
$$

This allows us to work with the un-deformed Leibniz rule,

$$
\begin{aligned}
& \text { "d } \mathbf{d}\left(" \omega \wedge_{\star_{N}} " \gamma\right)=\quad \text { "d " } \omega \wedge_{\star_{N}} " \gamma+(-1)^{|\omega|} " \omega \wedge_{\star_{N}} " \mathbf{d} " \gamma=
\end{aligned}
$$

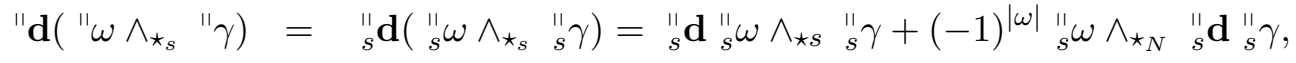

where ${ }_{s}^{\prime \prime} \omega$ is a homogeneous form of degree $|\omega|$. We consider that ${ }_{s}^{\prime \prime} \mathbf{d} \overline{\mathfrak{f}}^{\alpha_{s}}\left({ }_{s}^{\prime \prime} \omega\right)=\overline{\mathfrak{f}}^{\alpha_{s}}\left({ }_{s}^{\prime \prime} \mathbf{d}{ }_{s}^{\prime \prime} \omega\right)$ and ${ }_{s} \mathbf{d} \overline{\mathfrak{f}}_{\alpha_{s}}\left({ }_{s}^{\prime \prime} \omega\right)=$ $\overline{\mathfrak{f}}_{\alpha_{s}}\left({ }_{s}{ }_{s} \mathbf{d}{ }_{s}^{\prime \prime} \omega\right)$. Here, we emphasize that we shall use symbols "d or ${ }_{s} \mathbf{d}^{\mathbf{d}}$ depending on the fact if it is necessary to state explicitly, or not, a s-decomposition of corresponding d- / s-operators.

A corresponding s-adapted exterior differential calculus can be elaborated via exterior products between functions, i.e. 0 -forms and 1-forms, and respective s-adapted star deformations. This way, we generate the $C^{\infty}\left(\mathcal{M},{ }_{s} N\right)$-bimodule and resulting $\mathcal{A}_{s}^{\star}$-bimodule s-structure of spaces of 1 -forms, which for dyadic decompositions we denote $\Omega_{s}^{1}(\mathcal{M})=\Omega^{1}\left(\mathcal{M},{ }_{s}{ }_{s} N\right)$ and $\Omega_{\star s}^{1}(\mathcal{M})=\Omega_{\star}^{1}\left(\mathcal{M},{ }_{s} N\right)$.

For a s-adapted differential form nonassociative calculus, we present some important formulas when the s-components

$$
\begin{aligned}
& f \star_{N} " e^{j}=f \cdot " e^{j}-\frac{\kappa}{2} R^{j k b} \mathbf{e}_{k} f \cdot " \mathbf{e}_{b}=" \mathbf{e}^{j} \star_{N} f-" \mathbf{e}_{b} \star_{N} \kappa R^{j k b} \mathbf{e}_{k} f \\
& f \star_{s} " e^{j}=f \cdot " e^{j}-\frac{\kappa}{2} R^{j_{s} k_{s} b} \mathbf{e}_{k_{s}} f \cdot " \mathbf{e}_{b_{s}}=" \mathbf{e}^{j_{s}} \star_{s} f-" \mathbf{e}_{b_{s}} \star_{s} \kappa R^{j_{s} k_{s} b_{s}} \mathbf{e}_{k_{s}} f, \\
& f \star_{N} " \mathbf{e}_{a}=f \cdot " \mathbf{e}_{a}=" \mathbf{e}_{a} \star_{N} f \rightarrow f \star_{s} " \mathbf{e}_{a_{s}}=f \cdot " \mathbf{e}_{a_{s}}=" \mathbf{e}_{a_{s}} \star_{s} f
\end{aligned}
$$

are written with s-indices. We have also

$$
f \star_{N} \text { " } \mathbf{e}^{\alpha}=" \mathbf{e}^{\gamma} \star_{N}\left(f \delta_{\gamma}^{\alpha}-i \kappa \mathcal{R}_{\gamma}^{\alpha \beta} " \mathbf{e}_{\beta} f\right) \rightarrow f \star_{N} " \mathrm{e}^{\alpha_{s}}={ }^{\prime \mathbf{e}_{s}} \star_{s}\left(f \delta_{\gamma_{s}}^{\alpha_{s}}-i \kappa \mathcal{R}^{\alpha_{s} \beta_{s}} \gamma_{\gamma_{s}} \mathbf{e}_{\beta_{s}} f\right),
$$

when the absolute differential is star deformed in s-adapted form

$$
\begin{aligned}
& " \mathbf{d} f={ }^{\prime \prime} \mathbf{e}_{\beta} f " \mathbf{e}^{\beta}={ }^{\prime} \mathbf{e}_{\beta} f \star_{N} "{ }^{\prime} \mathbf{e}^{\beta}="{ }^{\beta} \star^{\beta}{ }_{N} " \mathbf{e}_{\beta} f= \\
& { }_{s}{ }_{s} \mathbf{d} f=" \mathbf{e}_{\beta_{s}} f " \mathbf{e}^{\beta_{s}}=" \mathbf{e}_{\beta_{s}} f \star_{s} " \mathbf{e}^{\beta_{s}}="{ }^{\beta^{\beta_{s}} \star_{s}}{ }{ } \mathbf{e}_{\beta_{s}} f \text {. }
\end{aligned}
$$

Such formulas can be re-defined in local coordinate bases still keeping, or not, a shell s-splitting of indices,

$$
\begin{aligned}
f \star_{N} d x^{j} & =f \cdot d x^{j}-\frac{i \kappa}{2} R^{j k b} \frac{\partial f}{\partial x^{k}} \cdot d p_{b}=d x^{j} \star_{N} f-d p_{b} \star_{N} i \kappa R^{j k b} \frac{\partial f}{\partial x^{k}} \rightarrow \\
f \star_{s} d x^{j_{s}} & =f \cdot d x^{j_{s}}-\frac{i \kappa}{2} R^{j_{s} k_{s} b_{s}} \frac{\partial f}{\partial x^{k_{s}}} \cdot d p_{b_{s}}=d x^{j_{s}} \star_{s} f-d p_{b_{s}} \star_{s} i \kappa R^{j_{s} k_{s} b_{s}} \frac{\partial f}{\partial x^{k_{s}}} \\
f \star_{N} d p_{a} & =f \cdot d p_{a}=d p_{a} \star_{N} f \rightarrow f \star_{s} d p_{a_{s}}=f \cdot d p_{a_{s}}=d p_{a_{s} \star_{s}} f .
\end{aligned}
$$

Above presented formalism can be extended to d-tensors involving N- and s-connections and quasi-Hopf s-structures. All such formulas are similar to those for N-adapted constructions in if " $\mathbf{e}_{\beta_{s}} \rightarrow$ "| $\mathbf{e}_{\beta}$ and $\star_{s} \rightarrow \star_{N}$, etc. In this work, we shall omit proofs and details if certain (non) associative formulas are s-analogs of respective $\mathrm{N}$-adapted ones. 


\subsection{2 s-tensor and dyadic quasi-Hopf star deformations}

We can deform always a s-adapted tensor product $\otimes_{C^{\infty}\left(\mathcal{M},{ }_{s}^{\prime \prime} N\right)}$ over $C^{\infty}\left(\mathcal{M},{ }_{s}^{\prime \prime} N\right)$ into a s-tensor product $\otimes_{\star_{s}}$ over s-algebra $\mathcal{A}_{s}^{\star}$. We follow the convention

$$
\begin{gathered}
{ }^{"} \mathbf{B} \otimes_{\star_{N}} \text { " } \mathbf{Q}=\overline{\mathfrak{f}}^{\alpha}(" \mathbf{B}) \otimes_{C^{\infty}(\mathcal{M}, N)} \overline{\mathfrak{f}}_{\alpha}(" \mathbf{Q})= \\
" \mathbf{B} \otimes_{\star_{s}} " \mathbf{Q}=\overline{\mathfrak{f}}^{\alpha_{s}}(" \mathbf{B}) \otimes_{C^{\infty}\left(\mathcal{M},{ }_{s} N\right)} \overline{\mathfrak{f}}_{\alpha_{s}}(" \mathbf{Q}),
\end{gathered}
$$

where the s-adapted twist on the s-tensors " $\mathbf{B}$ and " $\mathbf{Q}$ is taken via the Lie s-derivative and the nonassociativity for any $a \in \mathcal{A}_{N}^{\star}$, is involved as

$$
\begin{aligned}
& \left(" \mathbf{B} \star_{N} a\right) \otimes_{\star_{N}} \quad \mathbf{Q}={ }_{\phi_{1}} \mathbf{B} \otimes_{\star_{N}}\left({ }_{\phi_{2}} a \star_{N}{ }_{\phi_{3}} \mathbf{Q}\right)= \\
& \left(" \mathbf{B} \star_{s} a\right) \otimes_{\star_{s}} \quad \mathbf{Q}={ }_{\phi_{1}}^{\prime \prime} \mathbf{B} \otimes_{\star_{s}}\left({ }_{\phi_{2}} a \star_{s} \quad{ }_{\phi_{3}} \mathbf{Q}\right) \text {. }
\end{aligned}
$$

We cite for details on $\mathrm{N}$-adapted actions of associators $\phi_{1}, \phi_{2}$, and $\phi_{3}$ the section 2.6.2 in [3] having a straightforward s-structure re-formulation.

The N- and s-adapted star product of a function $f$ and s-components of partial derivatives

$$
\begin{aligned}
& f \star_{N} " \mathbf{e}_{i}=f \cdot " \mathbf{e}_{i}=" \mathbf{e}_{i} \star_{N} f \rightarrow f \star_{s} " \mathbf{e}_{i_{s}}=f \cdot "{ }^{\prime} \mathbf{e}_{i_{s}}=" \mathbf{e}_{i_{s} \star_{s}} f, \\
& f \star_{N} " e^{a}=f \cdot " e^{a}-\frac{i \kappa}{2} R^{j k a} \mathbf{e}_{j} f \cdot " \mathbf{e}_{k}=" e^{a} \star_{N} f-" \mathbf{e}_{j} \star_{N} i \kappa R^{a j k} \mathbf{e}_{k} f \rightarrow \\
& f \star_{s} " e^{a_{s}}=f \cdot " e^{a_{s}}-\frac{i \kappa}{2} R^{j_{s} k_{s} a_{s}} \mathbf{e}_{j_{s}} f \cdot "{ }^{\prime} \mathbf{e}_{k_{s}}=" e^{a_{s}} \star_{s} f-"{ }^{\prime} \mathbf{e}_{j_{s}} \star_{s} i \kappa R^{a_{s} j_{s} k_{s}} \mathbf{e}_{k_{s}} f .
\end{aligned}
$$

Here we nota that, for instance, " $\mathbf{e}_{i_{s}} \star_{s} f$ denotes the right s-adapted $\mathcal{A}_{s}^{\star}$-action on $V e c_{\star_{s}}$ (this is not on action of " $\mathbf{e}_{i_{s}}$ on the function $f$ ). For dyadic indices,

$$
f \star_{s} " \mathbf{e}_{\alpha_{s}}=" \mathbf{e}_{\gamma_{s} \star_{s}}\left(f \delta_{\alpha_{s}}^{\gamma_{s}}+i \kappa \mathcal{R}_{\alpha_{s}}^{\gamma_{s} \beta_{s}} " \mathbf{e}_{\beta_{s}} f\right) .
$$

In similar forms, using s-adapted star tensor products, we can extend the dyadic $\mathcal{A}_{s}^{\star}$-bimodule $V e c_{\star_{s}}$ of s-vector fields to the $\Omega_{\star}^{\natural}$-bimodule $V e c_{\star_{s}}^{\natural}=\operatorname{Vec}_{\star_{s}} \otimes_{\star_{s}} \Omega_{\star}^{\natural}\left(\mathcal{M},{ }_{s}^{\|} N\right)$. This way, we re-express the d-tensor and d-form $\star_{N}$-calculus from section 2 of [3] into a respective s-adapted one which allows $2+2+\ldots$ decoupling of fundamental geometric and physical important formulas (see next sections).

\section{Dyadic geometry of star deformed (non) symmetric s-metrics}

Nonassociative geometric models with star R-flux deformed symmetric and nonsymmetric metric structures and respective modified vacuum Einstein equations for the LC-connection are elaborated in Refs. [1, 2] in abstract and coordinate frames. The goal of this section is to re-define the generalizations with N-connection structure from section 3.3 of [3] in s-adapted frames which will be applied in next section for proofs of decoupling and integrability of nonassociative vacuum gravitational equations. The key constructions will be based on performing nonholonomic dyadic decompositions of (non) symmetric metric s-structures and respective canonical s-connection and nonholonomic constraints to LC-connection structures. Nonassociative canonical Riemann and Ricci s-tensors adapted to quasi-Hopf s-structure are defined and computed following methods from appendix $\mathrm{A}$.

\subsection{The geometry of phase space s-metrics and canonical s-connections}

The goal of this subsection is to study main properties of (non) symmetric metric and related linear connection structures which are generated by N- and s-adapted star deformations to quasi-Hopf structures on phase spaces $\mathcal{M}=\mathbf{T}_{\|}^{*} \mathbf{V}$. 


\subsubsection{Symmetric and nonsymmetric s-metric structures and star deformations}

A (pseudo) Riemannian symmetric metric on cotangent Lorentz bundle $T^{*} V$ is defined by a metric " $g=$ $\left\{\right.$ " $\left.g_{\alpha \beta}\right\} \in T T^{*} V \otimes T T^{*} V$, which for nonassociative generalizations of Einstein gravity is chosen to be of local signature $(+,+,+,-;+,+,+,-)$. Such a metric can be expressed in a nonholonomic dyadic form for shells $s=1,2,3,4$, i.e. as a s-metric on phase space $\mathcal{M}=\mathbf{T}_{\|}^{*} \mathbf{V}$, with respect to symmetric tensor products of s-bases " $\mathbf{e}^{\alpha_{s}} \in T_{s}^{*} \mathbf{T}_{\|}^{*} \mathbf{V}$ (8),

$$
\begin{aligned}
& g \rightarrow{ }_{s}^{\prime \prime} \mathbf{g}=\left(h_{1} " \mathbf{g}, v_{2} " \mathbf{g}, c_{3} " \mathbf{g}, c_{4} " \mathbf{g}\right) \in T \mathbf{T}_{\|}^{*} \mathbf{V} \otimes_{\star N} T \mathbf{T}_{\|}^{*} \mathbf{V} \\
& =\quad " \mathbf{g}_{\alpha_{s} \beta_{s}}\left(\begin{array}{c}
" \\
s
\end{array}\right) " \mathbf{e}^{\alpha_{s}} \otimes_{\star s} " \mathbf{e}^{\beta_{s}}=\left\{" \mathbf{g}_{\alpha_{s} \beta_{s}}=\left(" \mathbf{g}_{i_{1} j_{1}}, " \mathbf{g}_{a_{2} b_{2}}, " \mathbf{g}^{a_{3} b_{3}}, " \mathbf{g}^{a_{4} b_{4}}\right)\right\} .
\end{aligned}
$$

In the case of R-flux deformations to nonassociative geometry, symmetric metric transforms into symmetric and nonsymmetric ones [1, 2] (for nonholonomic N-adapted constructions, see section 3.3 of [3]). We use such s-adapted parameterizations (in brief, we shall write s-metrics)

$$
\begin{aligned}
& \text { symmetric: } \quad{ }_{\star s} \mathbf{g}=\left(h_{1}{ }_{\star s} \mathbf{g}, v_{2}{ }_{\star s} \mathbf{g}, c_{3}{ }_{\star s} \mathbf{g}, c_{4}{ }_{\star s}{ }^{\prime} \mathbf{g}\right) \\
& =\left\{{ }_{\star}{ }^{\prime \prime} \mathbf{g}_{\alpha_{s} \beta_{s}}={ }_{\star}{ }_{\star} \mathbf{g}_{\beta_{s} \alpha_{s}}=\left({ }_{\star}{ }_{\star} \mathbf{g}_{i_{1} j_{1}}={ }_{\star}{ }_{\star} \mathbf{g}_{j_{1} i_{1}},{ }_{\star}{ }_{\star} \mathbf{g}_{a_{2} b_{2}}={ }_{\star}{ }_{\star} \mathbf{g}_{b_{2} a_{2}},{ }_{\star}{ }_{\star} \mathbf{g}^{a_{3} b_{3}}={ }_{\star}{ }_{\star} \mathbf{g}^{b_{3} a_{3}},{ }_{\star}{ }_{\star} \mathbf{g}^{a_{4} b_{4}}={ }_{\star}{ }_{\star} \mathbf{g}^{b_{4} a_{4}}\right)\right\} \text {, } \\
& \text { and } \\
& \text { nonsymmetric: } \quad{ }_{\star s}^{\prime \prime} \mathfrak{g}=\left(h_{1}{ }_{\star s}{ }^{\prime \prime} \mathfrak{g}, v_{2}{ }_{\star s}{ }^{\prime \prime} \mathfrak{g}, c_{3}{ }_{\star s}{ }_{\star s} \mathfrak{g}, c_{4}{ }_{\star s}{ }^{\prime \prime} \mathfrak{g}\right)
\end{aligned}
$$

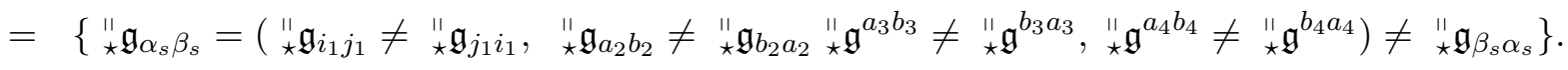

In coordinate bases, when "e ${ }^{\alpha_{s}} \rightarrow " e^{\alpha}=d^{\|} u^{\alpha} \in T^{*} T_{\|}^{*} V$ and we can omit the shell/dyadic label $s$, such star deformed metric structures can be considered generic in off-diagonal forms,

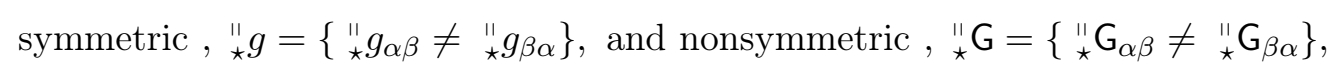

Here we note that for fixed $\mathrm{N}$-/ s-connection and respective coordinate structures, we have ${ }_{\star}^{\|} \mathbf{g}={ }_{\star s}{ }_{\star} \mathbf{g}={ }_{\star}^{\prime} g$ and ${ }_{\star} \mathfrak{g}={ }_{\star s} \mathfrak{g}={ }_{\star} \mathrm{k} G$, but with different block $(4 \times 4)+(4 \times 4),[(2 \times 2)+(2 \times 2)]+[(2 \times 2)+(2 \times 2)]$, and $8 \times 8$ matrices of coefficients when $\mathrm{N}$-adapted, s-adapted or local coordinate (co) frame decompositions are considered. In this paper, we follow the approach with quasi-Hopf s-algebras because it allows to elaborate on s-adapted diffeomorphisms and star nonholonomic deformations. This can be used for straightforward generalizations to classical and quantum physical and information theory models. We write $G$ for nonsymmetric metrics as in Section 5 of [2] but revise the notations and the formulas in s-adapted form and with boldface symbols and labels $N, s, \stackrel{\star}{\star}$, etc.

A s-metric ${ }_{s} \mathrm{~g}$ on a associative and commutative tangent Lorentz co-bundle with shell by shell dyadic decomposition, $\mathbf{T}_{s||}^{*} \mathbf{V}$, is a symmetric tensor which can be described by respective coefficients of s-adapted (8) and/or local coordinate co-bases,

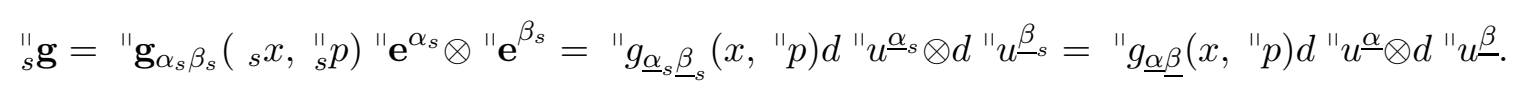

We use labels for local coordinates in the form (1) when certain indices are underlined in order to state that they are for some coefficients with respect to local coordinate (co) bases. On base manifold and total space we can consider general coordinate transforms which mix shell's coordinates, we can omit, for simplicity, s-parametrization for such coordinates. The coefficients from (1) are defined via frame transforms,

$$
" \mathbf{g}_{\alpha_{s} \beta_{s}}=" e^{\underline{\alpha}_{s}} \text { " } e_{\beta_{s}}^{\underline{\beta}_{s}} " g_{\underline{\alpha}_{s} \underline{\beta}_{s}}=" e e_{\alpha_{s}}^{\underline{\alpha}} " e^{\underline{\beta}}{ }_{\beta_{s}} " g_{\underline{\alpha} \underline{\beta}},
$$

relating respective block dyadic decompositions with off-diagonal matrices. For instance, with respect to local 
coordinate dual basis $d^{\prime \prime} u^{\underline{\alpha}}$, get such recurrent off-diagonal parameterizations:

$$
\begin{aligned}
& \text { "g } \underline{\alpha}_{1} \underline{\beta}_{1}\left(x^{k_{1}}\right)=\quad " g_{\underline{i}_{1} \underline{i}_{1}}=" g_{i_{1} i_{1}}=g_{i_{1} j_{1}}\left(x^{k_{1}}\right) \text {, with identification of indices for } s=1 \text {, } \\
& \text { " } g_{\underline{\alpha}_{2} \underline{\beta}_{2}}\left(x^{k_{1}}, x^{c_{2}}\right)=\left[\begin{array}{cc}
g_{i_{1} j_{1}}+g_{a_{2} b_{2}} N_{i_{1}}^{a_{2}} N_{j_{1}}^{b_{2}} & g_{a_{2} e_{2}} N_{i_{1}}^{e_{2}} \\
g_{a_{2} e_{2}} N_{i_{1}}^{e_{2}} & " g_{a_{2} b_{2}}
\end{array}\right] s=2 \text {, for } \\
& g_{a_{2} b_{2}}=g_{a_{2} b_{2}}\left(x^{k_{1}}, x^{c_{2}}\right), N_{i_{1}}^{a_{2}}=N_{i_{1}}^{a_{2}}\left(x^{k_{1}}, x^{c_{2}}\right) ;
\end{aligned}
$$

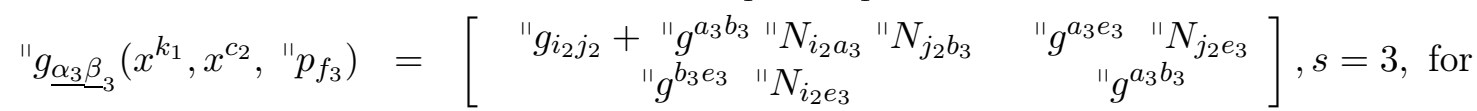

$$
\begin{aligned}
& \text { " } g_{i_{2} j_{2}}=" g_{i_{2} j_{2}}\left(x^{k_{1}}, x^{c_{2}}\right), " g^{a_{3} b_{3}}=" g^{a_{3} b_{3}}\left(x^{k_{1}}, x^{c_{2}}, " p_{f_{3}}\right), \\
& \text { " } N_{j_{2} e_{3}}="{ }^{\prime} N_{j_{2} e_{3}}\left(x^{k_{1}}, x^{c_{2}}, " p_{f_{3}}\right) \text {; } \\
& " g_{\underline{\alpha}_{4} \underline{\beta}_{4}}\left(x^{k_{1}}, x^{c_{2}}, " p_{f_{3}}, " p_{f_{4}}\right)=\left[\begin{array}{cc}
" g_{i_{3} j_{3}}+{ } g^{a_{4} b_{4}} " N_{i_{3} a_{4}} " N_{j_{3} b_{4}} & " g^{a_{3} e_{3}} " N_{j_{3} e_{4}} \\
& " g^{b_{4} e_{4}} " N^{a_{3} b_{3} e_{4}}
\end{array}\right], s=4 \text {, for } \\
& \text { " } g_{i_{3} j_{3}}="{ } g_{i_{3} j_{3}}\left(x^{k_{1}}, x^{c_{2}}, " p_{f_{3}}\right), " g^{a_{4} b_{4}}=" g^{a_{4} b_{4}}\left(x^{k_{1}}, x^{c_{2}}, " p_{f_{3}}, " p_{f_{4}}\right), \\
& \text { " } N_{i_{3} a_{4}}=" N_{i_{3} a_{4}}\left(x^{k_{1}}, x^{c_{2}}, " p_{f_{3}}, " p_{f_{4}}\right) \text {. }
\end{aligned}
$$

Equivalently, shell by shell, above formulas define s-adapted metric and N-connection coefficients are parameterized shell by shell in the form (15),

$$
\begin{aligned}
& \text { "} \mathbf{g}_{\alpha_{s} \beta_{s}}=\left[{ }^{\prime} \mathbf{g}_{i_{1} j_{1}}=g_{i_{1} j_{1}}\left(x^{k_{1}}\right), " \mathbf{g}_{a_{2} b_{2}}=g_{a_{2} b_{2}}\left(x^{k_{1}}, x^{c_{2}}\right),\right. \\
& \text { " } \left.\mathbf{g}^{a_{3} b_{3}}="{ }^{a_{3} b_{3}}\left(x^{k_{1}}, x^{c_{2}}, " p_{f_{3}}\right), " \mathbf{g}^{a_{4} b_{4}}=" g^{a_{4} b_{4}}\left(x^{k_{1}}, x^{c_{2}}, " p_{f_{3}}, " p_{f_{4}}\right)\right] \text {; }
\end{aligned}
$$

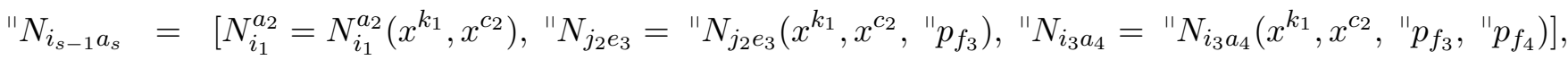

where the N-connection s-coordinate coefficients are chosen in the form (77). Such s-parameterizations can be used for proofs of a general decoupling and integration of nonholonomic gravitational and geometric flow systems of PDEs in modified gravity (non) commutative theories, see reviews [4, 5, 20, 21, 23.

\subsubsection{Canonical s-connections as distortions of dyadic LC-connections}

For nonholonomic distributions on phase space, we can define different d-connection structures following different geometric principles of adapting to respective N-connection structures. Using a s-metric ${ }_{s} \mathbf{g}$ (15), we can work in equivalent form with two different linear connections which are adapted or not to respective nonholonomic distributions:

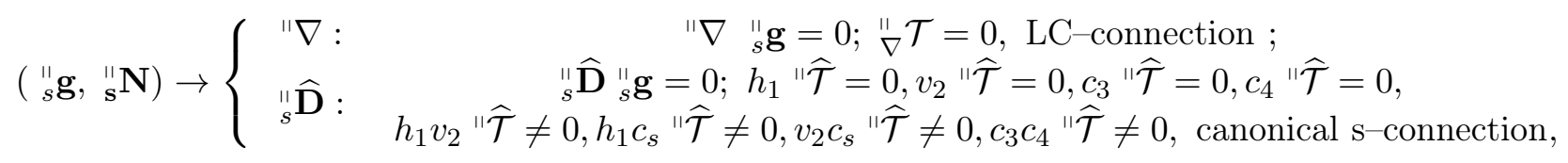

where ${ }_{s} \mid \widehat{\mathbf{D}}=\left(h_{1} " \widehat{\mathbf{D}}, v_{2} " \widehat{\mathbf{D}}, c_{3} " \widehat{\mathbf{D}}, c_{4} " \widehat{\mathbf{D}}\right)$, with dyadic (co) vertical splitting, is a s-connection adapted to a Nconnection structure ${ }_{s} \mathrm{~N}$. Such linear (affine) and nonlinear connections and their s-torsion are nonholonomic but commutative variants of nonassociative values introduced in appendix, see formulas (A.1) and (A.5). We use "hat" values in order to emphasize that such s-adapted values are determined by a s-metric structure following certain nonholonomic constraints involving (partial) zero torsion and metric compatibility conditions. The LC-connection " $\nabla$ is not a $\mathrm{d}$-/ or s-connection because it does not preserve the N-connection splitting and/or dyadic decompositions under parallel transports.

We provide here the s-adapted coefficients of the (associative and commutative) canonical s-connection ${ }_{s}^{\prime \prime} \widehat{\mathbf{D}}$ on $T \mathbf{T}_{\|}^{*} \mathbf{V}$, which is an example with trivial R-fluxes of nonassociative s-connection ${ }_{s}^{\|} \mathbf{D}^{\star}$ with respective 
dyadic decomposition (A.4) (see labeling of s-indices in those formulas):

$$
\begin{aligned}
& { }_{s} \widehat{\mathbf{D}}^{\mathrm{\mathbf {D }}}=\left\{" \widehat{\boldsymbol{\Gamma}}_{\alpha_{s} \beta_{s}}^{\gamma_{s}}=\left(\widehat{L}_{j_{1} k_{1}}^{i_{1}}, \widehat{L}_{b_{2} k_{1}}^{a_{2}}, \widehat{C}_{j_{1} c_{2}}^{i_{1}}, \widehat{C}_{b_{2} c_{2}}^{a_{2}} ; " \widehat{L}_{j_{2} k_{2}}^{i_{2}}, " \widehat{L}_{a_{3} k_{2}}^{b_{3}}, "{ }^{C^{2}} \widehat{C}_{j_{2}}^{i_{2} c_{3}},\right.\right. \\
& \text { " } \left.\left.\widehat{C}_{a_{3} b_{3}}^{c_{3}} ; \text { " } \widehat{L}_{j_{3} k_{3}}^{i_{3}}, " \widehat{L}_{a_{4} k_{3}}^{b_{4}}, " \text { " } C_{j_{3}}^{i_{3} c_{4}}, "{ }^{c_{a_{4} b_{4}}}\right)\right\} \text {, } \\
& \text { where } \widehat{L}_{j_{1} k_{1}}^{i_{1}}=\frac{1}{2} " g^{i_{1} r_{1}}\left(" \mathbf{e}_{k_{1}} " g_{j_{1} r_{1}}+\text { "e } \mathbf{e}_{j_{1}} " g_{k_{1} r_{1}}-" \mathbf{e}_{r_{1}} " g_{j_{1} k_{1}}\right) \text {, } \\
& \widehat{L}_{b_{2} k_{1}}^{a_{2}}={ } e_{b_{2}}\left(" N_{k_{1}}^{a_{2}}\right)+\frac{1}{2} " g^{b_{2} c_{2}}\left(" e_{k_{1}} " g_{b_{2} c_{2}}-" g_{d_{2} c_{2}} " e_{b_{2}} " N_{k_{1}}^{d_{2}}-" g_{d_{2} b_{2}} " e_{c_{2}} " N_{k_{1}}^{d_{2}}\right), \\
& \widehat{C}_{j_{1} c_{2}}^{i_{1}}=\frac{1}{2} " g^{i k} " e_{c} " g_{j k}, \quad \widehat{C}_{b_{2} c_{2}}^{a_{2}}=\frac{1}{2} " g^{a_{2} d_{2}}\left(" e_{c_{2}} " g_{b_{2} d_{2}}+" e_{b_{2}} " g_{c_{2} d_{2}}-" e_{d_{2}} " g_{b_{2} c_{2}}\right), \\
& \text { " } \widehat{L}_{a_{3} k_{2}}^{b_{3}}=" e^{b_{3}}\left(" N_{a_{3} k_{2}}\right)+\frac{1}{2} " g_{a_{3} c_{3}}\left(" e_{k_{2}} " g^{b_{3} c_{3}}-" g^{d_{3} c_{3}} " e^{b_{3}} " N_{d_{3} k_{2}}-" g^{d_{3} b_{3}} " e^{c} " N_{d_{3} k_{2}}\right), \\
& \text { " } \widehat{C}_{j_{2}}^{i_{2} c_{3}}=\frac{1}{2} " g^{i_{2} k_{2}} " e^{c_{3}} " g_{j_{2} k_{2}}, \quad "{ }^{C^{2}} \widehat{C}_{a_{3}} c_{3}=\frac{1}{2} " g_{a_{3} d_{3}}\left(" e^{c_{3}} " g^{b_{3} d_{3}}+" e^{b_{3}} " g^{c_{3} d_{3}}-" e^{d_{3}} " g^{b_{3} c_{3}}\right), \\
& \text { " } \widehat{L}_{a_{4} k_{3}}^{b_{4}}=" e^{b_{4}}\left(" N_{a_{4} k_{3}}\right)+\frac{1}{2} " g_{a_{4} c_{4}}\left(" e_{k_{3}} " g^{b_{4} c_{4}}-" g^{d_{4} c_{4}} " e^{b_{4}} " N_{d_{4} k_{3}}-" g^{d_{4} b_{4}} " e^{c_{4}} " N_{d_{4} k_{3}}\right),
\end{aligned}
$$

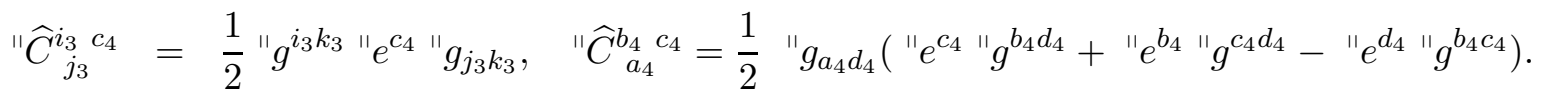

By straightforward computations with such coefficients (see similar details and proofs for $\mathbf{T}_{1}^{*} \mathbf{V}$ in [4, 5]), we can check that there are satisfied all necessary conditions from the definition of the canonical s-connection in (21). Introducing s-coefficients (22) in formulas (24) and (27), we can compute respective s-adapted coefficients for the canonical Ricci s-tensor, corresponding Ricci scalars and distortions from respective values determined by the LC-connection.

A LC-connection " $\nabla$ is not a d-connection and can not be expressed as a s-connection. Nevertheless, we can compute a canonical distortion relation to canonical s-connection,

$$
{ }_{s}^{\prime \prime} \widehat{\mathbf{D}}=" \nabla+{ }_{s} \widehat{\mathbf{Z}} \text {. }
$$

The distortion s-tensor, ${ }_{s}^{\prime} \widehat{\mathbf{Z}}=\left\{" \widehat{\mathbf{Z}}_{\beta_{s} \gamma_{s}}^{\alpha_{s}}\left[" \widehat{\mathbf{T}}_{\beta_{s} \gamma_{s}}^{\alpha_{s}}\right]\right\}$, is an algebraic combination of the coefficients the canonical torsion s-tensor ${ }_{s}^{\prime} \widehat{\mathcal{T}}=\left\{" \widehat{\mathbf{T}}_{\beta_{s} \gamma_{s}}^{\alpha_{s}}\right\}$ of ${ }_{s}^{\prime \prime} \widehat{\mathbf{D}}$. The canonical s-torsion is a commutative variant of the nonassociative d-torsion parameterized as in formulas (A.8), without star labels but with "hats" for canonical values. We shall provide explicit formulas for (non) associative canonical s-torsions in next sections. This distortion is determined by the s-connection coefficients and anholonomy coefficients " $w_{\alpha_{s} \beta_{s}}^{\gamma_{s}}$, see similar formulas (4) computed for s-frames " $\mathbf{e}_{\alpha_{s}}$ (8).

\subsubsection{Canonical nonholonomic Ricci and Einstein s-tensors}

For both type linear connections " $\nabla$ and ${ }_{s}^{\prime \prime} \widehat{\mathbf{D}}$ (21), we can define and compute in standard forms (as in metric-affine geometry) respective torsions, ${ }_{\nabla}^{\prime \prime} \mathcal{T}=0$ and ${ }_{s} \widehat{\mathcal{T}}$, and curvatures, ${ }_{\nabla} \mathcal{R}=\left\{{ }_{\nabla} R_{\beta \gamma \delta}^{\alpha}\right\}$ and ${ }_{s} \widehat{\mathcal{R}}=\left\{" \widehat{\mathbf{R}}_{\beta_{s} \gamma_{s} \delta_{s}}^{\alpha_{s}}\right\}$. Such general frame/coordinate and s-frame can defined and computed in with respect to arbitrary, coordinate, and/or N-/ s-adapted frames.

The canonical Ricci s-tensor ${ }_{s}^{\|} \widehat{\mathcal{R}} i c=\left\{" \widehat{\mathbf{R}}_{\beta_{s} \gamma_{s}}:={ }^{"} \widehat{\mathbf{R}}_{\alpha_{s} \beta_{s} \gamma_{s}}^{\gamma_{s}}\right\}$ and, for the LC-connection, "Ric= $\left\{" R_{\beta \gamma}:=" R_{\alpha \beta \gamma}^{\gamma}\right\}$ (we omit the label $\nabla$ writing formulas in not "boldface" forms and without shell labels if such formulas do not result in ambiguities) are defined and computed by a respective contracting the first and fours indices. The canonical s-tensor ${ }_{s} \widehat{\mathcal{R}} i c$ is characterized by s-adapted coefficients,

$$
\begin{aligned}
& \text { " } \widehat{\mathbf{R}}_{\beta_{s} \gamma_{s}}=\left\{" \widehat{R}_{h_{1} j_{1}}=" \widehat{\mathbf{R}}_{h_{1} j_{1} i_{1}}^{i_{1}}, " \widehat{P}_{j_{1} a_{2}}=-{ } \widehat{\mathbf{R}}^{i_{1}}{ }_{j_{1} i_{1} a_{2}}, " \text { " } \widehat{P}_{\star b_{2} k_{1}}=" \widehat{\mathbf{R}}_{b_{2} k_{1} c_{2}}^{c_{2}}, " \widehat{S}_{\star b_{2} c_{2}}=" \widehat{\mathbf{R}}_{b_{2} c_{2} a_{2}}^{a_{2}},\right.
\end{aligned}
$$

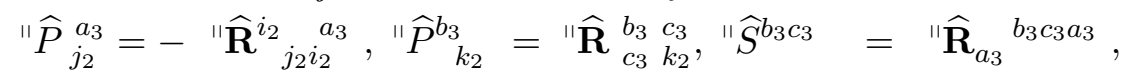

$$
\begin{aligned}
& \left." \widehat{P}_{j_{3}}^{a_{4}}=-" \widehat{\mathbf{R}}_{j_{3} i_{3}}^{i_{3} a_{4}}, " \widehat{P}_{k_{3}}^{b_{4}}=" \widehat{\mathbf{R}}_{c_{4} k_{3}}^{b_{4} c_{4}}, " \widehat{S}^{b_{4} c_{4}}=" \widehat{\mathbf{R}}_{a_{4}} b_{4} c_{4} a_{4}\right\},
\end{aligned}
$$


are defined as the associative commutative part (the component of order $(i \hbar)^{0}$ and $(i \kappa)^{0}$ of A.17) with scoefficients (A.15). Introducing in such formulas the canonical s-connection coefficients (22), we can express the values " $\widehat{\mathbf{R}}_{\beta_{s} \gamma_{s}}$ (24) in terms of the coefficients of s-metric and $\mathrm{N}-/$ s-connection. We note that in our nonholonomic dyadic approach ${ }_{s} \widehat{\mathcal{R}} i c$ is an associative and commutative variant of the nonassociative Ricci s-tensor ${ }_{s} \Re i c^{\star}=\left\{" \mathbf{R} i c_{\alpha_{s} \beta_{s}}^{\star}\right.$ (A.17) with s-adapted distributions parameterized in such forms that

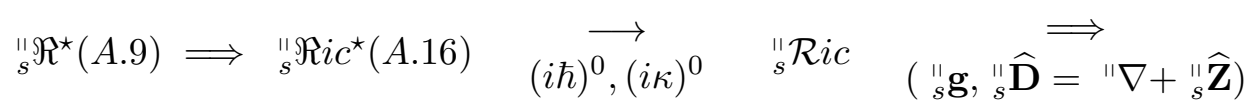

$$
\begin{aligned}
& \begin{array}{c}
{ }_{s} \widehat{\mathcal{R}} i c=\left\{\widehat{\mathbf{R}}_{\alpha_{s} \beta_{s}}\right\} \\
\text { on } \mathbf{T}^{*} \mathbf{V}
\end{array} \\
& \text { nonholonomic } \\
& \text { s-transforms \& lifts } \\
& \Uparrow \\
& R_{i j} \text { on } V
\end{aligned}
$$

Contracting the coefficients of inverse (s-) metric with respective coefficients of the canonical Ricci (s-) tensor, we can define two different scalar curvatures,

$$
\begin{aligned}
& { }_{s}{ }_{s} \widehat{\mathbf{R}} s c:=\quad{ } \mathbf{g}^{\alpha_{s} \beta_{s}} " \widehat{\mathbf{R}}_{\alpha_{s} \beta_{s}}="{ }^{i_{1} j_{1}} " \widehat{R}_{i_{1} j_{1}}+" g^{a_{2} b_{2}} " \widehat{R}_{a_{2} b_{2}}+" g_{a_{3} b_{3}} " \widehat{R}^{a_{3} b_{3}}+" g_{a_{4} b_{4}} " \widehat{R}^{a_{4} b_{4}} \\
& \text { and " } R:={ }^{\prime} \mathbf{g}^{\alpha \beta} " R_{\alpha \beta} .
\end{aligned}
$$

The modified Einstein equations for $\left({ }_{s}^{\|} \mathbf{g},{ }_{s}^{\|} \widehat{\mathbf{D}}\right)$ on $\mathbf{T}_{\|}^{*} \mathbf{V}$ with a nontrivial cosmological constant $" \lambda$ can be postulated using the same geometric principles as in GR (see, for instance, [24]) but extended to cotangent Lorentz bundles, see an axiomatic approach and details in [4, 5], in the form

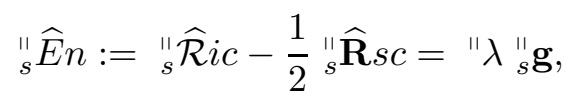

where ${ }_{s} \widehat{E} n=\left\{{ }_{s}^{\prime \prime} \widehat{E}_{\alpha_{s} \beta_{s}}\right\}$ is by definition the canonical Einstein s-tensor. Such forms of commutative nonholonomic modifications of vacuum gravitational Einstein equations are constructed via lifts of metrics and related linear connection $\nabla$, or possible other type d-connections, from Lorentz spacetime to it cotangent bundle and

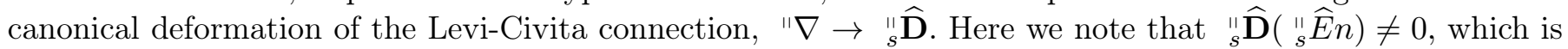
typical for theories with nonholonomic constraints 5 Using distortions of d-connections of type (23), we can always nonholonomically deform such systems of nonlinear PDEs into equivalent ones with ${ }_{s} \widehat{E} n \rightarrow{ }_{\nabla} E n$, when $" \nabla\left({ }_{\nabla}^{\|} E n\right)=0$. Such canonical s-distortions of geometric objects and physically important gravitational and geometric flows equations, are determined by respective distortions of the curvature and Ricci tensors,

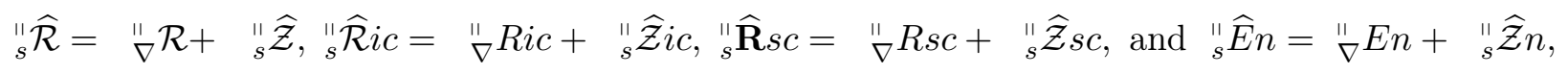

with corresponding distortion s-tensors ${ }_{\nabla} \widehat{\mathcal{Z}}$ and ${ }_{\nabla}^{\|} \widehat{\mathcal{Z}} i c$ determined by $\left({ }_{s} \| \widehat{\mathbf{Z}}\right.$, ${ }_{s} \mathbf{g}$ ) on a (pseudo) Riemannian phase background with " $\nabla$. We omit abstract and cumbersome s-adapted/ coordinate formulas for such geometric objects.

To construct generic off-diagonal solutions and study their physical properties in certain models of phase gravity, we can consider effective cosmological constants on any shell $s=1,2,3,4$ and/or for a h- $/ \mathrm{c}-$ decomposition. We shall use for star parametric (on $\hbar, \kappa$, and $\hbar \kappa$ ) deformations the solutions of associative/ commutative nonholonomic vacuum gravitational equations

$$
\text { " } \widehat{\mathbf{R}}_{\beta_{s} \gamma_{s}}={ }_{s}{ }_{s} \lambda " \mathbf{g}_{\beta_{s} \gamma_{s}} .
$$

These equations transform into (26) if ${ }_{s}^{\prime \prime} \lambda=" \lambda$ for all values of $s$. The goal of this work is to prove that nonassociative vacuum equations constructed as R-flux and star canonical s-deformations of (28) can be

\footnotetext{
${ }^{5}$ For instance, the conservation laws in nonholonomic Lagrange mechanics should be revised by introducing additional multiples associated to nonholonomic constraints of different type.
} 
decoupled and integrated in certain general form for quasi-stationary configurations (when in corresponding s-adapted variables the metric's s-coefficients and other fundamental geometric objects do not depend on time like coordinate $x^{4}=t$ ).

We conclude that in the associative and commutative case (with zero R-flux), the geometry of phase space $\mathbf{T}_{\|}^{*} \mathbf{V}$ can be described by a standard (pseudo) Riemannian geometry (we can consider momentum coordinates multiplied to complex unity). Equivalently, we can consider models with nonholonomic induced torsion by the N-connection structure on $\mathbf{T}_{\|}^{*} \mathbf{V}$ as we explained in details in [3]. In this work, we use nonholonomic dyadic decompositions when (from the same metric structure) we can derive both a canonical s-connection and the LC-connection, and can apply the AFCDM for constructing exact solutions. We note that all geometric constructions can be performed equivalently working with different geometric data ( "g, " $\nabla$ ) and $\left({ }_{s}^{\|} \mathbf{g},{ }_{s} \mathbf{N},{ }_{s}^{\|} \widehat{\mathbf{D}}\right)$.

\subsection{Nonassociative canonical s-connections and LC-configurations}

To provide physical motivations for star deformations (they can result both in noncommutative and noncommutative geometries) of commutative models of gravity and geometric flow theories and their physically important classes of solutions, we elaborate a formalism of nonassiative R-flux determined star s-adapted deformations of (non) symmetric metrics and (non) linear connections for phase spaces with quasi-Hopf sstructure.

\subsubsection{Conventions on parameterizing nonassociative R-flux star deformations}

We extend for nonholonomic dyadic decompositions the Conventions 1 and 2 analyzed in section 2.2.1 of [3] on constructing nonassociative geometries:

- Convention 1: R-flux star deformations to nonassocitative geometric models are elaborating in coordinate frames and beginning with a flat (co) fiber metric " $\eta$. In [1, 2], star products $\star$ are defined in terms of coordinate bases " $\partial$ and respective nonassociative generalizations of (pseudo) Riemann geometry are constructed in using R-flux deformations to symmetric, ${ }_{\star}^{\prime \prime} g$, and nonsymmetric, ${ }_{\star} \mathrm{G}$, starmetric structures and a related nonassociative variant of LC-connection $" \nabla^{\star}$. There are considered such star-deformations of geometric structures:

$$
(" \eta, " \partial, " \nabla) \rightarrow\left(\star, \mathcal{A}^{\star}, \underset{\star}{\|} g, \underset{\star}{\| \mathrm{G},}, " \partial " \nabla^{\star}\right),
$$

with possible adapting of constructions to quasi-Hopf algebras $\mathcal{A}^{\star}$, or other type algebraic and geometric structures. The nonassocitative gravity theories were elaborated to a level of vacuum gravitational equations ${ }_{\nabla}^{\|} R i c^{\star}=0$ on a target star deformed phase space. There were computed real (proportional to parameters products $\hbar \kappa$ ) R-flux contributions for projections on pseudo-Riemannian spacetime $V$. Such fundamental geometric and gravitational equations are formulated in terms of tensor and linear connection objects with coefficients computed with respect to coordinate (co) bases $d$ " $u^{\alpha}$ and " $\partial_{\alpha}$ and their star deformations. Technically, it is very difficult to decouple and solve in certain explicit exact/ parametric forms such systems of nonlinear PDEs encoding nonassociative R-flux contributions etc., and to elaborate on quantum deforming of exact/parametric generic off-diagonal solutions in such theories.

- Convention 2: Our main goal is to elaborate a nonassociative generalization of the AFCDM method which allows to construct exact solutions defined by generic off-diagonal symmetric and nonsymmetric metrics and generalized (non) linear connections. The coefficients of geometric objects for such solutions depend, in general, on all possible phase space and spacetime coordinates and various classes of off-diagonal solutions can be constructed in different modified noncommutative and commutative gravity theories, in particular, in GR. Such a geometric techniques can be formulated using respective nonholonomic frame and connection deformations to certain nonholonomic and dyadic N-/s-adapted structures 
resulting in systems of nonlinear PDEs which can be decoupled and integrated in certain general forms. In a series of recent our works (the first partner one is [3]), we elaborate and apply geometric methods when commutative theories are formulated in certain general nonholonomic frame forms and then deformed by star nonholonomic products constructed for " $\mathbf{e}_{\alpha}$ adapted to N-connection structure "N. We extend the conditions of Convention 2 in [3] to the case of star products (10) defined with nonholonomic dyadic decompositions on " $\mathbf{e}_{\alpha_{s}}$ with R-flux terms. There are computed respective star deformations of canonical s-adapted geometric objects into nonassociative ones, with symmetric, ${ }_{\star s} \mathbf{g}(16)$, and nonsymmetric, ${ }_{\star s} \mathfrak{g}$ (17), star s-metrics and canonical star s-connection ${ }_{s}^{\|} \mathbf{D}^{\star}$. The nonholonomic dyadic star deformations of geometric canonical s-structures are defined by such star transforms of geometric data:

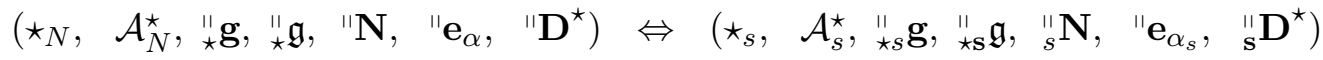

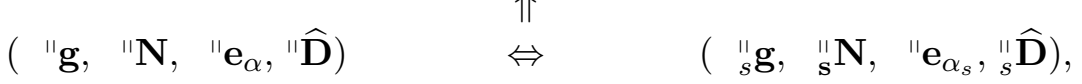

for ${ }^{\prime \prime} \mathbf{D}^{\star}=" \nabla^{\star}+" \widehat{\mathbf{Z}}^{\star}$ and ${ }_{s} \mathbf{D}^{\star}=" \nabla^{\star}+{ }_{s} \widehat{\mathbf{Z}}^{\star}$. This way, we prove in section 5 that there are certain general decoupling and integration properties of nonassociative vacuum Einstein equations in canonical nonholonomic s-variables, written in the form (28), for stationary configurations and parametric decompositions on $\hbar, \kappa$, and $\hbar \kappa$.

\subsubsection{Nonsymmetric metrics and s-metrics and their inverses}

We study s-adapted metric structures in nonassociative nonholonomic dyadic differential geometry and related star deformations of the LC-connections and canonical s-connections on phase spaces endowed with quasi-Hopf s-structures. A star metric symmetric s-tensor (16) can be represented in the form

$$
{ }_{\star s}^{\prime \prime} \mathbf{g}={ }_{\star}^{\|} \mathbf{g}_{\alpha_{s} \beta_{s} \star_{s}}\left(" \mathbf{e}^{\alpha_{s}} \otimes_{\star s} " \mathbf{e}^{\beta_{s}}\right) \in \Omega_{\star}^{1} \otimes_{\star s} \Omega_{\star}^{1} .
$$

There are considered real-valued s-adapted coefficients ${ }_{\star}^{\prime \prime} \mathbf{g}\left(" \mathbf{e}_{\alpha_{s}}, " \mathbf{e}_{\beta_{s}}\right)={ }_{\star}{ }_{\star} \mathbf{g}_{\alpha_{s} \beta_{s}}={ }_{\star}^{\prime \prime} \mathbf{g}_{\beta_{s} \alpha_{s}} \in \mathcal{A}_{s}^{\star}$, which follows

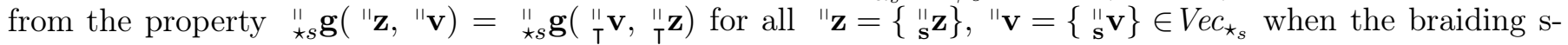
operator " $T$ " is defined as in formula (A.6). Such a s-metric is compatible with a star s-connection ${ }_{s} \mathbf{D}^{\star}$ (A.1) if the condition

$$
{ }_{s} \mathbf{D}^{\star}{ }_{\star s} \mathbf{g}=0
$$

is satisfied.

R-flux deformations result also in nonsymmetric metric structures [1, 2] and such geometric d- / s-objects have to be considered additionally to the symmetric metric. A nonsymmetric metric (18) on phase space $\mathbf{T}_{\|}^{*} \mathbf{V}$ can be defined in generic off-diagonal form with respect to local coordinate bases when

$$
{ }_{\star}^{\|} \mathrm{G}_{\alpha \beta}={ }_{\star}{ }_{\star} g_{\alpha \beta}-i \kappa \mathcal{R}^{\tau \xi}{ }_{\alpha} " \partial_{\xi}{ }_{\star} g_{\beta \tau} .
$$

In general, such coefficients define a nonsymmetric $8 \times 8$ matrix. With respect to a dyadic s-adapted basis " $\mathbf{e}_{\xi_{s}}$ and tensor products of their dual, a nonsymmetric s-metric structure (17) can be parameterized in a $[(2 \times 2)+(2 \times 2)]+[(2 \times 2)+(2 \times 2)]$ block form,

$$
{ }_{\star}^{\|} \mathfrak{g}_{\alpha_{s} \beta_{s}}={ }_{\star}^{\|} \mathbf{g}_{\alpha_{s} \beta_{s}}-i \kappa \overline{\mathcal{R}}_{\alpha_{s}}^{\tau_{s} \xi_{s}} " \mathbf{e}_{\xi_{s}}{ }_{\star} \mathbf{g}_{\beta_{s} \tau_{s}} .
$$

In these formulas, the R-flux coefficients re-defined in s-adapted form $\overline{\mathcal{R}}^{\tau_{s} \xi_{s}} \alpha_{s}$ determine a star nonsymmetric generalization of the commutative s-metric (19) with respective frame transforms from coordinate cobases and inversely, then the metric structure can be represented equivalently in s-adapted and/or (not adapted) coordinate forms,

$$
{ }_{\star s}^{\prime \prime} \mathfrak{g}={ }_{\star} " \mathfrak{g}_{\alpha_{s} \beta_{s}} \star_{s}\left(" \mathbf{e}^{\alpha_{s}} \otimes_{\star s} " \mathbf{e}^{\beta_{s}}\right)={ }_{\star}^{"} \mathrm{G}_{\alpha \beta} \star\left(d " u^{\alpha} \otimes_{\star} d " u^{\beta}\right),
$$


where ${ }_{\star} \mathfrak{g}_{\alpha_{s} \beta_{s}} \neq{ }_{\star} " \mathfrak{g}_{\beta_{s} \alpha_{s}}$ and ${ }_{\star}^{\|} \mathrm{G}_{\alpha \beta} \neq{ }_{\star} " \mathrm{G}_{\beta \alpha}$.

For quasi-Hopf configurations, a holonomic formalism of constructing inversions of matrices in $\mathcal{A}^{\star}$ is elaborated in section 5.2 of [2]. It allows us to compute the inverse matrix $" \bar{G}^{-1}=\left\{{ }_{\star} " G^{\alpha \beta}\right\}$ of a matrix " $\bar{G}=\left\{{ }_{\star}^{\|} G_{\alpha \beta}\right\}$ as a solution of algebraic equations ${ }_{\star}^{\|} \mathrm{G}^{\alpha \beta} \cdot{ }_{\star}{ }^{\prime \prime} G_{\beta \gamma}={ }_{\star}{ }_{\star} G_{\gamma \beta} \cdot{ }_{\star}{ }^{\prime \prime} G^{\beta \alpha}=\delta_{\beta}^{\alpha}$. Such matrix formulas are derived using geometric series, when

$$
{ }_{\star}^{" G} \mathrm{G}^{\alpha \beta}=" g^{\alpha \beta}-i \kappa " g^{\alpha \tau} \mathcal{R}_{\tau}^{\mu \nu}\left(\partial_{\mu} " g_{\nu \varepsilon}\right) " g^{\varepsilon \beta}+O\left(\kappa^{2}\right),
$$

which $\mathcal{R}^{\mu \nu}{ }_{\tau}$ constructing via nonholonomic transforms. Similar formulas can be written in block d-forms for matrices constructed from s-adapted coefficients of a nonsymmetric star s-metric ${ }_{\star}^{\|} \mathfrak{g}_{\alpha_{s} \beta_{s}}$ and, respectively, of a symmetric star d-metric $\underset{\star}{\|} \mathbf{g}_{\alpha_{s} \beta_{s}}$.

\subsubsection{Star deformed LC-connections and canonical s-connections}

We define star deformations of the two linear connections structure (21) using a star d-metric ${ }_{\star} \mathrm{g}$ and work in equivalent forms with respective two different linear connections:

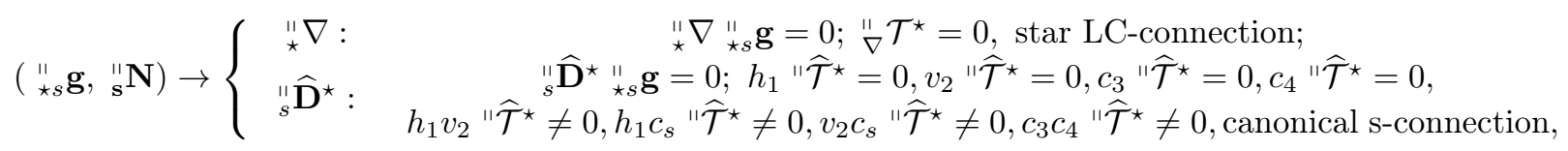

where ${ }_{s} \widehat{\mathbf{D}}^{\star}=\left(h_{1} " \widehat{\mathbf{D}}^{\star}, v_{2} " \widehat{\mathbf{D}}^{\star}, c_{3}\right.$ " $\left.\widehat{\mathbf{D}}^{\star}, c_{4} " \widehat{\mathbf{D}}^{\star}\right)$ encodes a nonholonomic dyadic horizontal and (co) vertical splitting, is a s-connection adapted to a nonlinear s-connection structure ${ }_{s} \mathbf{N}^{6}$ Such a canonical s-connection satisfies the metricity conditions (30) but contains certain "mixing shells" nontrivial torsion coefficients of " $\widehat{\mathcal{T}}^{\star}$ even "pure" shell torsions vanish in s-adapted (co) frames " $\mathbf{e}_{\alpha_{s}}(\underline{8})$ and $" \mathbf{e}^{\alpha_{s}}$ (9).

In above formulas, the nonassociative metric compatibility of both linear connections is stated as in (30) and the star canonical s-torsion components are parameterized following decompositions (A.8), when

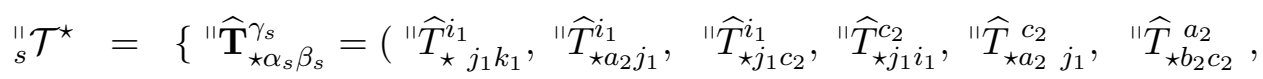

$$
\begin{aligned}
& \text { " } \left.\left.\widehat{T}_{\star j_{2}}^{i_{2} a_{3}}, \quad " \widehat{T}_{\star a_{3} j_{2} i_{2}}, \quad " \widehat{T}_{\star c_{3} j_{2}}^{a_{3}}, " \text { " } \widehat{T}_{\star a_{3}}^{b_{3} c_{3}}, " \text { " } \widehat{T}_{\star j_{3}}^{i_{3} a_{4}}, " \text { " } \widehat{T}_{\star a_{4} j_{3} i_{3}}, " \widehat{T}_{\star c_{4} j_{3}}^{a_{4}},{ }{ } \widehat{T}_{\star a_{4}}^{b_{4} c_{4}}\right)\right\} .
\end{aligned}
$$

The coefficients for such canonical s-torsion (A.8) are computed

$$
\begin{aligned}
& " \widehat{T}_{\star j_{1} k_{1}}^{i_{1}}=\quad " \widehat{L}_{\star j_{1} k_{1}}^{i_{1}}-{ }^{i_{1}} \widehat{L}_{\star k_{1} j_{1}}^{i_{1}}, " \widehat{T}_{\star a_{2} j_{1}}^{i_{1}}=" \widehat{C}_{\star a_{2} j_{1}}^{i_{1}}, \quad " \widehat{T}_{\star j_{1} c_{2}}^{i_{1}}=" \widehat{C}_{\star j_{1} c_{2}}^{i_{1}}, " \widehat{T}_{\star j_{1} i_{1}}^{c_{2}}=-{ }^{c_{2}} \Omega_{\star j_{1} i_{1}}^{c_{2}}, \\
& \text { " } \widehat{T}_{\star a_{2} j_{1}}^{c_{2}}=" \widehat{L}_{\star a_{2} j_{1}}^{c_{2}}-" e_{a_{2}}\left(" N_{\star j_{1}}^{c_{2}}\right), \quad " \widehat{T}_{\star b_{2} c_{2}}^{a_{2}}=" \widehat{C}_{\star b_{2} c_{2}}^{a_{2}}-"{ }^{a_{\star}} \widehat{C}_{\star c_{2} b_{2}}^{a_{2}} ; \\
& " \widehat{T}_{\star j_{2}}^{i_{2} a_{3}}={ }^{2} \widehat{C}_{\star j_{2}}^{i_{2} a_{3}}, \quad " \widehat{T}_{\star a_{3} j_{2} i_{2}}=-{ } \Omega_{\star a_{3} j_{2} i_{2}}, \quad " \widehat{T}_{\star c_{3} j_{2}}^{a_{3}}=" \widehat{L}_{\star c_{3} j_{2}}^{a_{3}}-" e^{a_{3}}\left("{ } N_{\star c_{3} j_{2}}\right), \\
& " \widehat{T}_{\star a_{3}}^{b_{3} c_{3}}={ }^{\prime} \widehat{C}_{\star a_{3}}^{b_{3} c_{3}}-{ }^{\prime} \widehat{C}_{\star a_{3}}^{c_{3} b_{3}}, \\
& \text { " } \widehat{T}_{\star j_{3}}^{i_{3} a_{4}}={ }^{\prime} \widehat{C}_{\star j_{3}}^{i_{3} a_{4}}, \quad " \widehat{T}_{\star a_{4} j_{3} i_{3}}=-{ }{ } \Omega_{\star a_{4} j_{3} i_{3}}, \quad " \widehat{T}_{\star c_{4} j_{3}}^{a_{4}}=" \widehat{L}_{\star c_{4} j_{3}}^{a_{4}}-"{ }^{a_{4}}\left({ }^{a_{4}}\left(" N_{\star c_{4} j_{3}}\right),\right. \\
& " \widehat{T}_{\star a_{4}}^{b_{4} c_{4}}={ }^{\prime} \widehat{C}_{\star a_{4}}^{b_{4} c_{4}}-{ }^{\prime} \widehat{C}_{\star a_{4}}^{c_{4} b_{4}} .
\end{aligned}
$$

The next step is to find the dependence of canonical torsion s-coefficients (36) on s-metric and s-connection coefficients by introducing a set of s-adapted Christoffel symbols

$$
{ }_{[0]}^{\prime \prime} \widehat{\boldsymbol{\Gamma}}_{\star \gamma_{s} \alpha_{s} \beta_{s}}={ }_{\star}^{\|} \mathbf{g}_{\gamma_{s} \tau_{s}}{ }_{[0]}^{\|} \widehat{\boldsymbol{\Gamma}}_{\star \beta_{s} \alpha_{s}}^{\tau_{s}},
$$

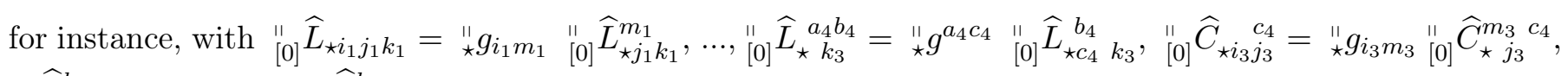
${ }_{[0]}^{\|} \widehat{C}_{\star}^{b_{4} e_{4} c_{4}}={ }_{\star}^{\|} g^{a_{4} e_{4}}{ }_{[0]}^{\|} \widehat{C}_{\star}^{b_{4} e_{4}} c_{4}$, where s-components of a star deformation of canonical s-connection (22) are

\footnotetext{
${ }^{6}$ We shall write in brief for such formulas that they are with a h1-v2-c3-c4 decomposition.
} 
computed with respect to nonholonomic s-bases, using dyadic shell N-connection coefficients ${ }_{s} \mathbf{N}=\left\{" N_{a_{s} i_{1}}\right\}$ and the symmetric star s-metric for ${ }_{\star}^{\|} \mathrm{g}_{\alpha_{s} \beta_{s}}$ (16),

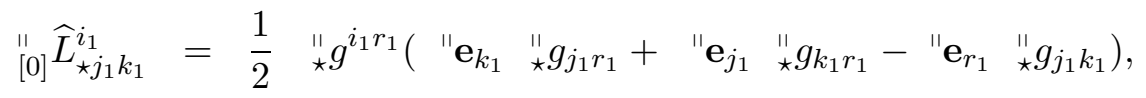

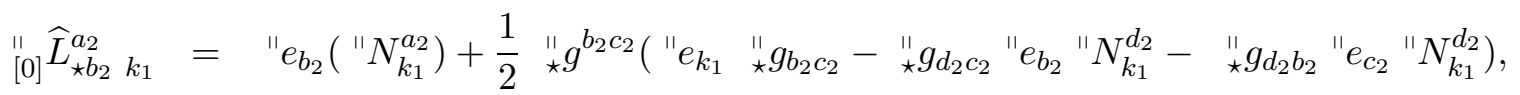

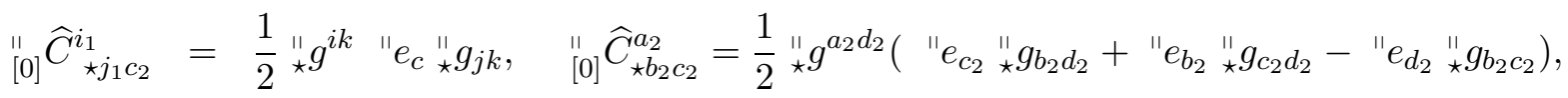

$$
\begin{aligned}
& { }_{[0]}^{\|} \widehat{L}_{\star a_{3} k_{2}}^{b_{3}}=" e^{b_{3}}\left(" N_{a_{3} k_{2}}\right)+\frac{1}{2}{ }_{\star}{ }_{\star} g_{a_{3} c_{3}}\left(" e_{k_{2}}{ }_{\star} g^{b_{3} c_{3}}-{ }_{\star}{ }_{\star} g^{d_{3} c_{3}} " e^{b_{3}} " N_{d_{3} k_{2}}-{ }_{\star} g^{d_{3} b_{3}} " e^{c} " N_{d_{3} k_{2}}\right),
\end{aligned}
$$

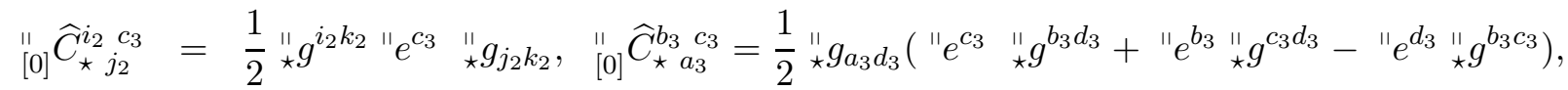

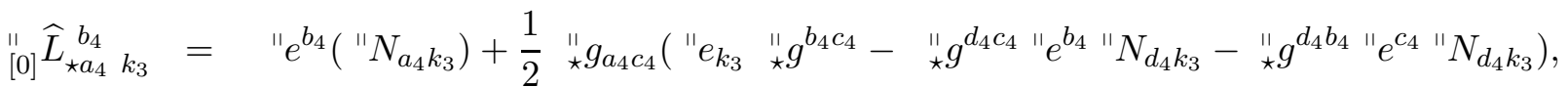

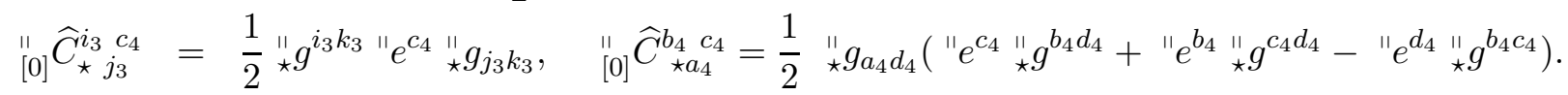

We parameterize R-flux s-adapted contributions (being proportional to $\kappa$ ) using s-adapted frames and symmetric s-metric coefficients,

$$
{ }_{[1]}^{\|} \widehat{\boldsymbol{\Gamma}}_{\star \alpha_{s} \beta_{s} \mu_{s}}=\frac{1}{2} \overline{\mathcal{R}}_{\mu_{s}}^{\xi_{s} \tau_{s}}\left({ }^{"} \mathbf{e}_{\xi_{s}} " \mathbf{e}_{\alpha_{s}}{ }_{\star} \mathbf{g}_{\beta_{s} \tau_{s}}+"{ }^{\prime \prime} \mathbf{e}_{\xi_{s}} " \mathbf{e}_{\beta_{s}}{ }_{\star} \mathbf{g}_{\alpha_{s} \tau_{s}}\right) .
$$

In result, we define in complete parametric form the $\mathrm{N}$-adapted coefficients of the star canonical d-connection $" \widehat{\mathbf{D}}^{\star}=\left\{" \widehat{\boldsymbol{\Gamma}}_{\star \alpha \beta}^{\gamma}\right\}$ considering a s-decomposition used torsion s-tensors (A.4),

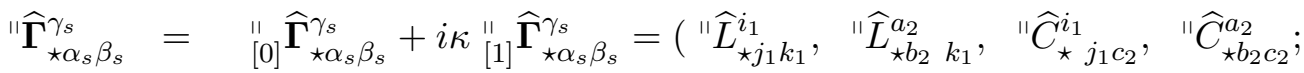

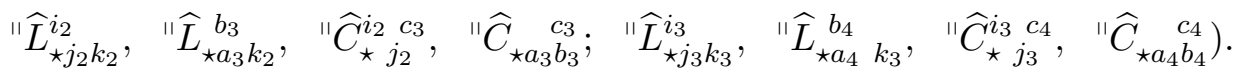

The nonsymmetric s-metric ${ }_{\star}^{\prime \prime} \mathfrak{g}_{\alpha_{s} \beta_{s}}(\underline{32})$ is involved following the condition

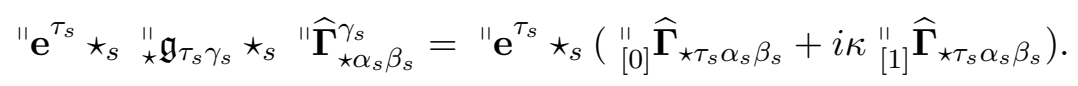

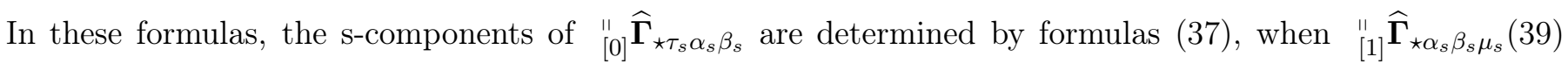
involve s-compositions of ${ }_{\star}^{\|} \mathbf{g}_{\beta_{s} \tau_{s}}$.

For nonassociative LC-configurations, formulas similar to (40) were considered in [2]. Following our system of notations, we can extract the coefficients $" \nabla^{\star}=\left\{" \Gamma_{\star \beta \alpha}^{\gamma}\right\}$ with respect to local coordinate dual frames " $\partial_{\tau}$,

$$
{ }_{\star}^{\prime \prime G} G_{\gamma \nu} \star " \Gamma_{\star \alpha \beta}^{\nu}=\frac{1}{2}\left(" \partial_{\beta}{ }_{\star}^{\prime \prime} g_{\alpha \gamma}+" \partial_{\alpha}{ }_{\star}^{\prime \prime} g_{\beta \gamma}-" \partial_{\gamma}{ }_{\star} g_{\alpha \beta}+i \kappa \mathcal{R}_{\gamma}^{\xi \tau}\left(" \partial_{\xi} " \partial_{\beta}{ }_{\star}^{\prime \prime} g_{\alpha \tau}+" \partial_{\xi} " \partial_{\alpha}{ }_{\star}^{\prime \prime} g_{\beta \tau}\right)\right) \text {. }
$$

In such formulas, it is used the off-diagonal nonsymmetric metric ${ }_{\star}^{\prime \prime} G_{\alpha \beta}$ computed as in (31). All formulas can be rewritten in s-adapted form if " $\partial_{\alpha} \rightarrow "{ }^{\prime} \mathbf{e}_{\alpha_{s}}$. We can compute also s-adapted coefficients of nonassociative star deformed $\nabla$ and star deformation of the associative and commutative canonical distortion s-relation (23),

$$
{ }_{s}^{" 1} \widehat{\mathbf{D}}^{\star}=" \nabla^{\star}+{ }_{\star s} \widehat{\mathbf{Z}} \text {. }
$$

The s-adapted coefficients of distortion s-tensor, ${ }_{s \star}^{\|} \widehat{\mathbf{Z}}=\left\{" \widehat{\mathbf{Z}}_{\star \beta_{s} \gamma_{s}}^{\alpha_{s}}\left[" \widehat{\mathbf{T}}_{\star \beta_{s} \gamma_{s}}^{\alpha_{s}}\right]\right\}$ can be computed as an algebraic combination of the coefficients of the nonassociative canonical s-tensor ${ }_{s} \mathcal{T}^{\star}=\left\{\right.$ " $\left.\widehat{\mathbf{T}}_{\star \beta_{s} \gamma_{s}}^{\alpha_{s}}\right\}$ for s-components (36) computed using formulas (37) and (38). 
Using nonholonomic dyadic decompositions, we can describe any model of nonassociative phase geometry determined by a nonsymmetric s-metric structure ${ }_{\star}^{\|} \mathfrak{g}_{\alpha_{s}} \beta_{s}$. For any given N-connection and R-flux s-coefficients data, we can consider only the symmetric star s-metric ${ }_{\star}^{\prime \prime} \mathrm{g}_{\alpha_{s}} \beta_{s}$, see formulas (32). Such a nonassociative geometry with symmetric and nonsymmetric s-metrics can be described equivalently both in terms of the star LC-connection $" \nabla^{\star}$ and/or the star canonical s-connection ${ }_{s} \widehat{\mathbf{D}}^{\star}$. To construct solutions of physically important systems of PDEs, it is convenient to work with nonholonomic dyadic canonical geometric data $\left({ }_{\star s}^{\|} \mathfrak{g},{ }_{s}^{\|} \widehat{\mathbf{D}}^{\star}\right)$. Then we can redefine the constructions in terms of star deformed LC-configurations using sdistortions (41). In certain important cases, we can extract such nonassociative LC-configurations imposing some zero s-torsion conditions

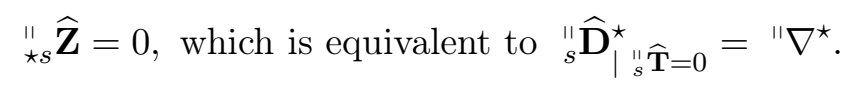

Even we can find nontrivial solutions which result in zero coefficients of (36), when " $\widehat{\mathbf{T}}_{\star \beta_{s} \gamma_{s}}^{\alpha_{s}}=0$, which is equivalent to (42), the anholonomy coefficients " $w_{\alpha_{s} \beta_{s}}^{\gamma_{s}}$ may be not zero (formulas (4) can be written for any (dual) s-shell). So, in general, we work with nonholonomic s-frames and involved (non) symmetric s-metrics can be written in local coordinate forms as generic off-diagonal matrices. Such matrices can not be diagonalized by coordinate transforms on a finite spacetime or phase space region if the anholonomy coefficients do not vanish. This criteria works in all types of nonassociative / noncommutative geometric models and their constraints to commutative nonholonomic configurations.

Nonassociative fundamental geometric s-objects from (29) can be decomposed on parameters $\hbar, \kappa$, and $\hbar \kappa$ which is important to compute R-flux deformations of associative and commutative geometric models. In appendix A.3. we provide respective formulas for such decompositions of nonassociative symmetric and nonsymmetric metrics and canonical s-connections.

\subsection{Nonassociative parametric decompositions of canonical Riemann and Ricci s-tensors}

We show how nonholonomic dyadic star deformations of important s-objects can be performed in parametric form but omit cumbersome formulas for further h1-v2-c3-c4 decompositions.

\subsubsection{Star and parametric deformed canonical curvature s-tenors}

The nonassociative canonical Riemann s-tensor can be computed by introducing the star canonical sconnection coefficients " $\widehat{\boldsymbol{\Gamma}}_{\star \alpha_{s} \beta_{s}}^{\gamma_{s}}$ (40) in formulas (A.13). In "hat" s-adapted variables, we have

$$
\begin{aligned}
& " \widehat{\Re}_{\alpha_{s} \beta_{s} \gamma_{s}}^{\star \mu_{s}}={ }_{1}^{\| \widehat{\Re}^{\star \mu_{s}}}{ }_{\alpha_{s} \beta_{s} \gamma_{s}}+{ }_{2}^{\|} \widehat{\Re}_{\alpha_{s} \beta_{s} \gamma_{s}}^{\star \mu_{s}} \text {, where }
\end{aligned}
$$

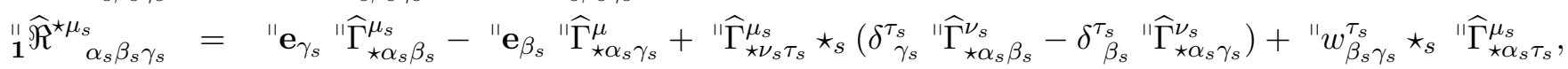

$$
\begin{aligned}
& { }_{2}^{\|} \widehat{\Re}_{\alpha_{s} \beta_{s} \gamma_{s}}^{\star \mu_{s}}=i \kappa " \widehat{\Gamma}_{\star \nu_{s} \tau_{s}}^{\mu_{s} \star_{s}}\left(\mathcal{R}_{\gamma_{s}}^{\tau_{s} \xi_{s}} " \mathbf{e}_{\xi_{s}} " \widehat{\Gamma}_{\star \alpha_{s} \beta_{s}}^{\nu_{s}}-\mathcal{R}_{\beta_{s}}^{\tau_{s} \xi_{s}} " \mathbf{e}_{\xi_{s}} " \widehat{\Gamma}_{\star \alpha_{s} \gamma_{s}}^{\nu_{s}}\right) \text {. }
\end{aligned}
$$

Further h1-v2-c3-c4 decompositions in s-adapted coefficients ("hat" variants of (A.15)), can be performed using formulas (38) and (39).

Parametric decompositions of the star canonical s-connections, see (A.23) and (A.24), can be computed in explicit s-form

$$
" \widehat{\boldsymbol{\Gamma}}_{\star \alpha_{s} \beta_{s}}^{\gamma_{s}}={ }_{[0]}^{\|} \widehat{\boldsymbol{\Gamma}}_{\star \alpha_{s} \beta_{s}}^{\nu_{s}}+i \kappa{ }_{[1]}^{\|} \widehat{\boldsymbol{\Gamma}}_{\star \alpha_{s} \beta_{s}}^{\nu_{s}}={ }_{[00]}^{\|} \widehat{\Gamma}_{* \alpha_{s} \beta_{s}}^{\nu_{s}}+{ }_{[01]}^{\prime \prime} \widehat{\Gamma}_{* \alpha_{s} \beta_{s}}^{\nu_{s}}(\hbar)+{ }_{[10]}^{\prime \prime} \widehat{\Gamma}_{* \alpha_{s} \beta_{s}}^{\nu_{s}}(\kappa)+{ }_{[11]}^{\prime \prime} \widehat{\Gamma}_{* \alpha_{s} \beta_{s}}^{\nu_{s}}(\hbar \kappa)+O\left(\hbar^{2}, \kappa^{2}, \ldots\right) .
$$

Then, introducing such parametric formulas in (43), we compute

$$
\| \widehat{\Re}_{\alpha_{s} \beta_{s} \gamma_{s}}^{\star \mu_{s}}={ }_{[00]}^{\|} \widehat{\Re}_{\alpha_{s} \beta_{s} \gamma_{s}}^{\star \mu_{s}}+{ }_{[01]}^{\|} \widehat{\Re}_{\alpha_{s} \beta_{s} \gamma_{s}}^{\star \mu_{s}}(\hbar)+{ }_{[10]}^{\| \widehat{\Re}_{\alpha_{s} \beta_{s} \gamma_{s}}^{\star \mu_{s}}}(\kappa)+{ }_{[11]}^{\| \widehat{\Re}_{\alpha_{s} \beta_{s} \gamma_{s}}^{\star \mu_{s}}}(\hbar \kappa)+O\left(\hbar^{2}, \kappa^{2}, \ldots\right) .
$$


To include in such a scheme nonholonomic dyadic decompositions of (non) commutative canonical Riemann s-tensors and their star deformations into nonassociative ones we can fix such nonholonomic s-distributions on $\mathcal{M}=\mathbf{T}_{s \mid l}^{*} \mathbf{V}$ when the values ${ }_{[00]}^{\| 0{ }^{\star \Re_{s}}}{ }_{\alpha_{s} \beta_{s} \gamma_{s}}$ define a "hat" variant of "not star deformed" curvature s-tensor for the canonical s-connection " $\widehat{\Gamma}_{\star \alpha_{s}}^{\mu_{s}}$ (21) . We omit cumbersome explicit formulas for the s-coefficients up till order $\hbar, \kappa$ and $\hbar \kappa$, for above geometric s-objects, because such values are not used in this paper.

\subsubsection{Parametric decomposition of star canonical Ricci s-tensors and their distortions}

Contracting on the fist and forth indices in formulas (43) and above formulas with parametric decompositions for the star canonical s-connection $" \widehat{\Gamma}_{\star \alpha_{s}}^{\mu_{s}} \gamma_{s}$, we define and compute the s-components the nonassociative canonical Ricci d-tensor as a "hat" variant of (A.16),

$$
\begin{aligned}
& { }_{\mathbf{s}} \widehat{\Re} i c^{\star}=" \widehat{\mathbf{R}} i c_{\alpha_{s} \beta_{s} \star_{s}}^{\star}\left(" \mathbf{e}^{\alpha_{s}} \otimes_{\star s} " \mathbf{e}^{\beta_{s}}\right) \text {, where } \\
& \text { " } \widehat{\mathbf{R}} i c_{\alpha_{s} \beta_{s}}^{\star}:={ }_{s} \widehat{\Re} i c^{\star}\left(" \mathbf{e}_{\alpha_{s}}, " \mathbf{e}_{\beta_{s}}\right)=\left\langle " \widehat{\mathbf{R}} i c_{\mu_{s} \nu_{s}}^{\star} \star_{s}\left(" \mathbf{e}^{\mu_{s}} \otimes_{\star_{s}} "{ }^{\nu_{s}}\right), \quad " \mathbf{e}_{\alpha_{s}} \otimes_{\star \mathbf{s}}{ } " \mathbf{e}_{\beta_{s}}\right\rangle_{\star_{s}} \text {. }
\end{aligned}
$$

The s-adapted coefficients are

$$
\begin{aligned}
& " \widehat{\mathbf{R}} i c_{\alpha_{s} \beta_{s}}^{\star}={ }^{\| \widehat{\Re}_{\alpha_{s} \beta_{s} \mu_{s}}^{\star \mu_{s}}}={ }_{[00]}^{\|} \widehat{\mathbf{R}} i c_{\alpha_{s} \beta_{s}}^{\star}+{ }_{[01]}^{\|} \widehat{\mathbf{R}} i c_{\alpha_{s} \beta_{s}}^{\star}(\hbar)+{ }_{[10]}^{\|} \widehat{\mathbf{R}} i c_{\alpha_{s} \beta_{s}}^{\star}(\kappa) \\
& +{ }_{[11]}^{\prime \prime} \widehat{\mathbf{R}} i c_{\alpha_{s} \beta_{s}}^{\star}(\hbar \kappa)+O\left(\hbar^{2}, \kappa^{2}, \ldots\right), \text { where } \\
& { }_{[00]}^{\| \prime} \widehat{\mathbf{R}} i c_{\alpha_{s} \beta_{s}}^{\star}={ }_{[00]}^{\|} \widehat{\Re}_{\alpha_{s} \beta_{s} \mu_{s}}^{\star \mu_{s}},{ }_{[01]}^{\prime \prime} \widehat{\mathbf{R}} i c_{\alpha_{s} \beta_{s}}^{\star}={ }_{[01]}^{\|} \widehat{\Re}_{\alpha_{s} \beta_{s} \mu_{s}}^{\star \mu_{s}}, \\
& { }_{[10]}^{\| 1} \widehat{\mathbf{R}} i c_{\alpha_{s} \beta_{s}}^{\star}={ }_{[10]}^{\|} \widehat{\Re}_{\alpha_{s} \beta_{s} \mu_{s}}^{\star \mu_{s}},{ }_{[11]}^{\prime \prime} \widehat{\mathbf{R}} i c_{\alpha_{s} \beta_{s}}^{\star}={ }_{[11]}^{\|} \widehat{\Re}_{\alpha_{s} \beta_{s} \mu_{s}}^{\star \mu_{s}}
\end{aligned}
$$

In appendix A.3.3, we show how to compute the parametric [00],[01],[10], and [11] s-coefficients from (45).

The h1-v2-c3-c4 splitting of a canonical Ricci s-connection is given by a corresponding "hat" variant of (A.17),

$$
\begin{aligned}
& " \widehat{\mathbf{R}} i c_{\alpha_{s} \beta_{s}}^{\star}=\left\{" \widehat{R}_{\star h_{1} j_{1}}=" \widehat{\Re}_{\star h_{1} j_{1} i_{1}}^{i_{1}}, \quad " \widehat{P}_{\star j_{1} a_{2}}=-" \widehat{\Re}_{\star j_{1} i_{1} a_{2}}^{i_{1}}, " \widehat{P}_{\star b_{2} k_{1}}=" " \widehat{\Re}_{\star b_{2} k_{1} c_{2}}^{c_{2}}, " \text { " } \widehat{S}_{\star b_{2} c_{2}}=" \widehat{\Re}_{\star b_{2} c_{2} a_{2}}^{a_{2}},\right. \\
& " \widehat{P}_{\star j_{2}}^{a_{3}}=-" \widehat{\Re}_{\star j_{2} i_{2}}^{i_{2}} a_{3}, " \widehat{P}_{\star k_{2}}^{b_{3}}=" \widehat{\Re}_{\star c_{3} k_{2}}^{b_{3} c_{3}}, " \widehat{S}_{\star}^{b_{3} c_{3}}=" \widehat{\Re}_{\star a_{3}} b_{3} c_{3} a_{3}, \\
& \left." \widehat{P}_{\star j_{3}}^{a_{4}}=-" \widehat{\Re}_{\star}^{i_{3} j_{3} i_{3}}, " \widehat{P}_{\star k_{3}}^{b_{4}}=" \widehat{\Re}_{\star c_{4} k_{3} b_{3}}^{b_{3}}, " \widehat{S}_{\star}^{b_{4} c_{4}}=" \widehat{\Re}_{\star a_{4}} b_{4} c_{4} a_{4}\right\} .
\end{aligned}
$$

Each component of (46) can be decomposed in respective [00], [01], [10] and [11] terms, for instance,

$$
\begin{aligned}
& { }_{[00]}^{\prime \prime} \widehat{\mathbf{R}} i c_{\alpha_{s} \beta_{s}}^{\star}=\left\{{ }_{[00]}^{\prime \prime} \widehat{R}{ }_{\star h_{1} j_{1}}={ }_{[00]}^{\|} \widehat{\Re}_{\star h_{1} j_{1} i_{1}}^{i_{1}},{ }_{[00]}^{\prime \prime} \widehat{P}_{\star j_{1} a_{2}}=-{ }_{[00]}^{\| 0} \widehat{\Re}_{\star}^{i_{1}}{ }_{j_{1} i_{1} a_{2}}, \quad{ }_{[00]}^{\prime \prime} \widehat{P}_{\star b_{2} k_{1}}={ }_{[00]}^{\| 0} \widehat{\Re}_{\star b_{2} k_{1} c_{2}}^{c_{2}}, \ldots\right\}, \\
& { }_{[01]}^{\|} \widehat{\mathbf{R}} i c_{\alpha_{s} \beta_{s}}^{\star}=\left\{\ldots,-{ }_{[01]}^{\|} \widehat{\Re}_{\star j_{2} i_{2}}^{i_{2}} a_{3}, \quad{ }_{[01]}^{\|} \widehat{P}_{\star k_{2}}^{b_{3}}={ }_{[01]}^{\| \Re_{\star c_{3}} k_{2}},{ }_{[01]}^{b_{3} c_{3}} \widehat{S}_{\star}^{b_{3} c_{3}}={ }_{[01]}^{\| \Re_{\star a_{3}} b_{3} c_{3} a_{3}}, \ldots\right\}
\end{aligned}
$$

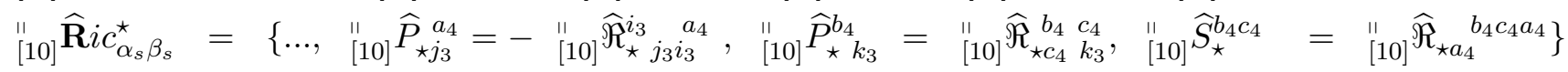

$$
\begin{aligned}
& { }_{[11]}^{\prime \prime} \widehat{\mathbf{R}} i c_{\alpha_{s} \beta_{s}}^{\star}=\left\{{ }_{[11]}^{\prime \prime} \widehat{R}_{\star h_{1} j_{1}}={ }_{[11]}^{\|} \widehat{\Re}_{\star h_{1} j_{1} i_{1}}^{i_{1}},{ }_{[11]}^{\prime \prime} \widehat{P}_{\star j_{1} a_{2}}=-{ }_{[11]}^{\|} \widehat{\Re}_{\star}^{i_{1}}{ }_{j_{1} i_{1} a_{2}}, \quad{ }_{[11]}^{\prime \prime} \widehat{P}_{\star b_{2} k_{1}}={ }_{[11]}^{\|} \widehat{\Re}_{\star b_{2} k_{1} c_{2}}^{c_{2}}, \ldots\right\} .
\end{aligned}
$$

We can arrange the nonholonomic distributions on phase space that ${ }_{[00]}^{\|} \widehat{\mathbf{R}} i c_{\alpha_{s} \beta_{s}}={ } \widehat{\mathbf{R}}_{\alpha_{s} \beta_{s}}(\underline{24})$ are determined by associative and commutative s-adapted canonical s-connection (24) but other $[01,10,11]:=$ $\lceil\hbar, \kappa\rceil$ components contain nonassociative and noncommutative contributions from star product deformations, which can be real or complex ones. In parametric form, we can express the star s-deformed Ricci s-tensor in the form

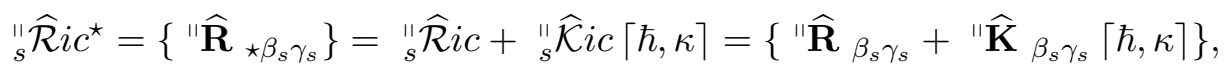

where $\left.{ }_{s} \widehat{\mathcal{K}} i c=\left\{" \widehat{\mathbf{K}}_{\beta_{s} \gamma_{s}}\lceil\hbar, \kappa\rceil\right\}\right\}$ encode nonassociative parametric deformations of the canonical Ricci stenor. If we take an associative and commutative solution ${ }_{\star} " \mathrm{~g}_{\alpha_{s} \beta_{s}}$ of nonholonomic dyadic vacuum Einstein 
equations (28), we can compute certain as R-flux and star canonical s-deformations to ${ }_{\star} \mathfrak{g}_{\tau_{s}} \gamma_{s}$. In section 6 , we shall generate parametric solutions for some classes of stationary nonassociative s-metrics ${ }_{\star} \mathfrak{g}_{\tau_{s}} \gamma_{s}$ when

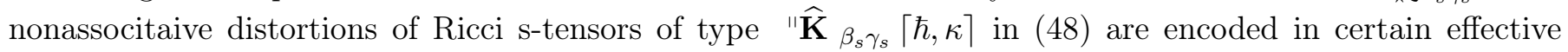
sources and/or cosmological constants, see subsection 4.2, Using respective formulas for parametric splitting of the nonassociative canonical Ricci s-tensor presented in appendix A.3.3 (see respective formulas (A.28), (A.29) and (A.31),

$$
" \widehat{\mathbf{K}}_{\beta_{s} \gamma_{s}}={ }_{[01]}^{\prime \prime} \widehat{\mathbf{R}} i c_{\beta_{s} \gamma_{s}}^{\star}+{ }_{[10]}^{\|} \widehat{\mathbf{R}} i c_{\beta_{s} \gamma_{s}}^{\star}+{ }_{[11]}^{\|} \widehat{\mathbf{R}} i c_{\beta_{s} \gamma_{s}}^{\star},
$$

where " $\widehat{\mathbf{R}}_{\beta_{s} \gamma_{s}}={ }_{[00]} \widehat{\mathbf{R}} i c_{\beta_{s} \gamma_{s}}^{\star}$ A.27) can be computted using formulas for commutative canonical Ricci s-tensors presented in our previous works [40, 21, 20, 19].

In our nonholonomic frame approach, we can elaborate (non) commutative and (non) associative theories in equivalent forms using different classes of linear connection structures defined by corresponding adapting to $\mathrm{N}-$ /s- structures. The canonical s-connection ${ }_{s}^{\prime \prime} \widehat{\mathbf{D}}^{\star}$ allows to decouple and solve in some general off-diagonal forms (exactly, or with dependence on certain parameters) star generalizations of (modified) Einstein equations as we prove in sections 5 and 6 . In another turn, the LC-connection " $\nabla^{\star}$ is a standard one used in GR and various MGTs. If both type of geometric constructions are determined by the same (non) associative metric s-structure $\left({ }_{\star}^{\|} \mathfrak{g}_{\tau_{s} \gamma_{s}}\right){ }_{\star}{ }_{\star} \mathbf{g}_{\alpha_{s} \beta_{s}}$, we can re-define mutually the geometric/ physical theories via corresponding frame transforms and connections deformations.

Introducing star distortions of the canonical s-connection, ${ }_{s}^{\prime \prime} \widehat{\mathbf{D}}^{\star}=" \nabla^{\star}+{ }_{\star s} \widehat{\mathbf{Z}}_{\mathbf{Z}}$ (41) into formulas for " $\widehat{\Re} i c^{\star}$ (44), we can compute the s-distortions of the nonassocitative canonical Ricci scalar and Ricci s-tensors (see formulas (27) for commutative s-configurations). In nonholonomic dyadic form, we obtain

$$
{ }_{s}^{\|} \widehat{\mathcal{R}} s c^{\star}={ }_{\nabla}^{\|} \mathcal{R} s c^{\star}+{ }_{\nabla}^{\| s} \widehat{\mathcal{Z}} s c^{\star} \text { and }{ }_{s}^{\|} \widehat{\Re} i c^{\star}={ }_{\nabla}^{\|} \Re i c^{\star}+{ }_{\nabla}^{\| s} \widehat{\mathcal{Z}} i c^{\star},
$$

with corresponding distortion tensors $\nabla^{\| s} \widehat{\mathcal{Z}}^{\star}$ and ${ }_{\nabla}^{\| s} \widehat{\mathcal{Z}} i c^{\star}$, where left labels state that we consider canonical s-deformations from the nonassociative LC-connection. We can extract also LC-configurations if impose additionally the zero torsion conditions (42).

\section{Nonassociative nonholonomic dyadic vacuum Einstein equations}

The goal of this section is to reformulate in nonholonomic dyadic s-adapted canonical variables the nonassociative vacuum Einstein equations formulated for quasi-Hopf structures [2]. In a similar form, we can consider s-adapted constructions for nonassociative geometric objects and equations from [1] but we omit such details in this work. In [3], we generalized that nonassociative geometric approach for N-adapted configurations with R-flux and star modifications of vacuum phase space gravitational equations with a nontrivial cosmological constant. We develop a nonholonomic geometric approach for deriving fundamental physical equations following geometric principles (in our case, with nonsymmetric metrics and generalized connections) as in [24, 4, 5]. In this paper, such associative and commutative vacuum gravitational are formulated using the canonical s-connection (28). Nonassociative modifications are encoded in parametric distortions of the canonical Ricci s-tensor (48).

\subsection{Nonassociative vacuum Einstein equations with (non) symmetric s-metrics}

Following Convention 2 (29) and using formulas for fundamental geometric s-objects (for (non) associative s-metrics, see (15), (16) and (17); for s-connections, see (23) and (41); and distortions of s-connections, see 
(27) and (50) ) and respective geometric s-distortions, we can work with such equivalent geometric data:

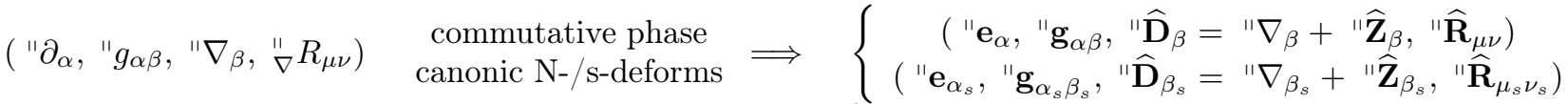

$$
\begin{aligned}
& \text { nonassoc. canonic } \Longrightarrow\left\{\left(\text { ( } \mathbf{e}_{\alpha},{ }_{\star}{ }_{\star} \mathfrak{g}_{\alpha \beta}, " \widehat{\mathbf{D}}_{\beta}^{\star}=" \nabla_{\beta}^{\star}+{ }_{\star}{ }_{\star} \widehat{\mathbf{Z}}_{\beta}, " \widehat{\mathbf{R}} i c_{\mu \nu}^{\star}\right)\right.
\end{aligned}
$$

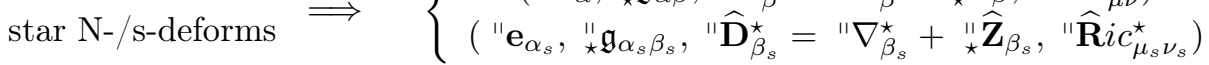

Using the canonical s-connection, we shall be able to decouple nonassociative vacuum gravitational equations in a parametric form which is similar to the case of associative (non) commutative gravity, see section 5 .

\subsubsection{Parametric decomposition of nonsymmetric star deformed s-metrics}

For any nonsymmetric s-metric ${ }_{\star}^{\prime \prime} \mathfrak{g}_{\alpha_{s} \beta_{s}}={ }_{\star} \mathfrak{g}_{\alpha_{s} \beta_{s}}^{[0]}+{ }_{\star} \mathfrak{g}_{\alpha_{s} \beta_{s}}^{[1]}(\kappa)$ (see formulas (17)) and (A.20), we can define and compute the symmetric part,

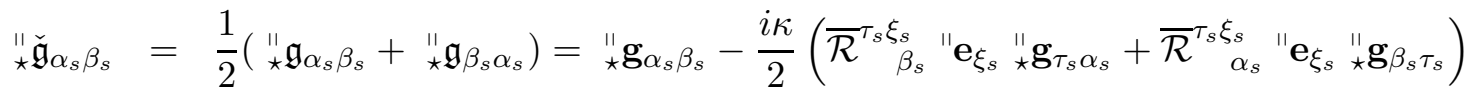

$$
\begin{aligned}
& ={ }_{\star} \check{\mathfrak{g}}_{\alpha_{s} \beta_{s}}^{[0]}+{ }_{\star} \check{\mathfrak{g}}_{\alpha_{s} \beta_{s}}^{[1]}(\kappa),
\end{aligned}
$$

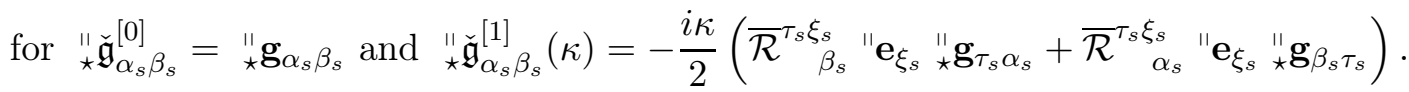

For nonholonomic dyadic decompositions, see formulas (16) and (32). The anti-symmetric part of a s-metric can be written with respect to s-frames when it is defined by R-fluxes,

$$
\begin{aligned}
& { }_{\star}^{\|} \mathfrak{a}_{\alpha_{s} \beta_{s}}:=\quad \frac{1}{2}\left({ }_{\star}{ }_{\star} \mathfrak{g}_{\alpha_{s} \beta_{s}}-{ }_{\star}{ }_{\star} \mathfrak{g}_{\beta_{s} \alpha_{s}}\right)=\frac{i \kappa}{2}\left(\begin{array}{c}
\overline{\mathcal{R}}^{\tau_{s} \xi_{s}} \\
\beta_{s}
\end{array}\right. \\
& ={ }_{\star}^{\|} \mathfrak{a}_{\alpha_{s} \beta_{s}}^{[1]}(\kappa)=\frac{1}{2}\left({ }_{\star}^{\|} \mathfrak{g}_{\alpha_{s} \beta_{s}}^{[1]}(\kappa)-{ }_{\star} " \mathfrak{g}_{\beta_{s} \alpha_{s}}^{[1]}(\kappa)\right) .
\end{aligned}
$$

We state ${ }_{\star} \mathfrak{a}_{\alpha \beta}^{[0]}=0$ if we consider nonassociative star deformations of commutative theories with symmetric metrics,

$$
{ }_{\star}^{\prime \prime} \mathfrak{g}_{\alpha_{s} \beta_{s}}={ }_{\star} \check{\mathfrak{g}}_{\alpha_{s} \beta_{s}}+{ }_{\star} " \mathfrak{a}_{\alpha_{s} \beta_{s}} .
$$

In geometric models with star deformation, we have to apply a more sophisticate procedure for computing inverse metrics. In Appendix A.3.1, we outline most important formulas for the case nonholonomic dyadic decompositions, see formulas (A.22), etc. The symmetric and nonsymmetric parts of a nonsymmetric s-metric with parametric decompositions can be written similarly to

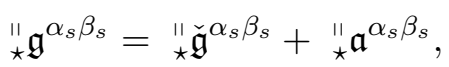

when, in general, ${ }_{\star}^{\|} \check{\mathfrak{g}}^{\alpha_{s} \beta_{s}}$ is not the inverse to ${ }_{\star}^{\prime \prime} \check{\mathfrak{g}}_{\alpha_{s} \beta_{s}}$ and ${ }_{\star}^{\prime \prime} \mathfrak{a}^{\alpha_{s} \beta_{s}}$ is not inverse to ${ }_{\star} \mathfrak{a}_{\alpha_{s} \beta_{s}}$. The inverse symmetric and nonsymmetric values are computed respectively:

$$
\begin{aligned}
& { }_{\star} \check{\mathfrak{g}}^{\alpha_{s} \beta_{s}}=\frac{1}{2}\left({ }_{\star} \check{\mathfrak{g}}^{\alpha_{s} \beta_{s}}+{ }_{\star} \check{\mathfrak{g}}^{\beta_{s} \alpha_{s}}\right) \\
& =\frac{1}{2}\left({ }_{\star}{ }_{\star} \mathbf{g}^{\alpha_{s} \beta_{s}}+{ }_{\star}{ }_{\star} \mathbf{g}^{\beta_{s} \alpha_{s}}\right)
\end{aligned}
$$

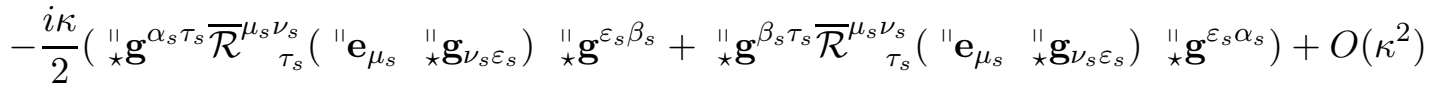

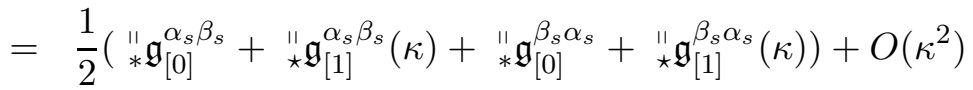




$$
\begin{aligned}
& ={ }_{*}^{\prime \prime} \mathfrak{g}_{[0]}^{\alpha_{s} \beta_{s}}+\frac{1}{2}\left({ }_{*} \mathfrak{x}_{[1]}^{\alpha_{s} \beta_{s}}(\kappa)+{ }_{*} \mathfrak{g}_{[1]}^{\beta_{s} \alpha_{s}}(\kappa)\right)+O\left(\kappa^{2}\right)={ }_{*}{ }_{*} \mathfrak{g}_{[0]}^{\alpha_{s} \beta_{s}}+{ }_{*} \check{\mathfrak{g}}_{[1]}^{\alpha_{s} \beta_{s}}(\kappa)+O\left(\kappa^{2}\right) \\
& ={ }_{*}^{\prime \prime} \mathfrak{g}_{[00]}^{\alpha_{s} \beta_{s}}+{ }_{*} \mathfrak{q}_{[01]}^{\alpha_{s} \beta_{s}}(\hbar)+{ }_{*} \mathfrak{\mathfrak { g }}_{[10]}^{\alpha_{s} \beta_{s}}(\kappa)+{ }_{*} \mathfrak{\mathfrak { g }}_{[11]}^{\alpha_{s} \beta_{s}}(\hbar \kappa)+O\left(\hbar^{2}, \kappa^{2}\right) \text {, for } \\
& { }_{*}^{\prime \prime} \check{\mathfrak{g}}_{[0]}^{\alpha_{s} \beta_{s}}={ }_{*}{ }_{*}^{\mathfrak{g}_{[s} \beta_{s}},{ }_{*}{ }_{*} \check{\mathfrak{g}}_{[1]}^{\alpha_{s} \beta_{s}}(\kappa)={ }_{*} \mathfrak{g}_{[1]}^{\alpha_{s} \beta_{s}}(\kappa),{ }_{*}{ }_{*} \mathfrak{g}_{[0]}^{\alpha_{s} \beta_{s}}={ }_{*}{ }_{*} \mathfrak{g}_{[0]}^{\beta_{s} \alpha_{s}}, \\
& \text { and }{ }_{\star} \mathfrak{a}^{\alpha_{s} \beta_{s}}=\frac{1}{2}\left({ }_{\star} \check{\mathfrak{g}}^{\alpha_{s} \beta_{s}}-{ }_{\star} \check{\mathfrak{g}}^{\beta_{s} \alpha_{s}}\right) \\
& =\frac{1}{2}\left({ }_{\star}{ }_{\star} \mathbf{g}^{\alpha_{s} \beta_{s}}-{ }_{\star}{ }_{\star} \mathbf{g}^{\beta_{s} \alpha_{s}}\right) \\
& -\frac{i \kappa}{2}\left({ }_{\star}{ }_{\star} \mathbf{g}^{\alpha_{s} \tau_{s}} \overline{\mathcal{R}}_{\tau_{s}}^{\mu_{s} \nu_{s}}\left({ }^{\prime \prime} \mathbf{e}_{\mu_{s}}{ }_{\star}{ }_{\star} \mathbf{g}_{\nu_{s} \varepsilon_{s}}\right){ }_{\star}{ }_{\star} \mathbf{g}^{\varepsilon_{s} \beta_{s}}-{ }_{\star} " \mathbf{g}^{\beta_{s} \tau_{s}} \overline{\mathcal{R}}_{\tau_{s}}^{\mu_{s} \nu_{s}}\left("{ }_{\mu_{\mu_{s}}}{ }_{\star} \mathbf{g}_{\nu_{s} \varepsilon_{s}}\right) \quad{ }_{\star} \mathbf{g}^{\varepsilon_{s} \alpha_{s}}\right)+O\left(\kappa^{2}\right)
\end{aligned}
$$

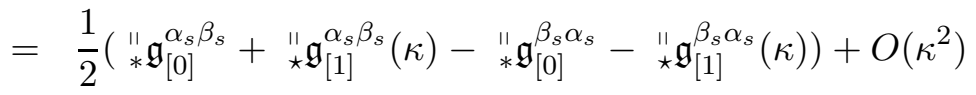

$$
\begin{aligned}
& =\frac{1}{2}\left({ }_{\star} \mathfrak{g}_{[1]}^{\alpha_{s} \beta_{s}}(\kappa)-{ }_{\star} \mathfrak{g}_{[1]}^{\beta_{s} \alpha_{s}}(\kappa)\right)+O\left(\kappa^{2}\right)={ }_{\star} \mathfrak{a}_{[1]} \mathfrak{a}_{[1]}^{\alpha_{s} \beta_{s}}(\kappa)+O\left(\kappa^{2}\right) \\
& ={ }_{*}^{\prime \prime} \mathfrak{a}_{[10]}^{\alpha_{s} \beta_{s}}(\kappa)+{ }_{*} \mathfrak{a}_{[11]}^{\alpha_{s} \beta_{s}}(\hbar \kappa)+O\left(\hbar^{2}, \kappa^{2}\right) \text {, for }
\end{aligned}
$$

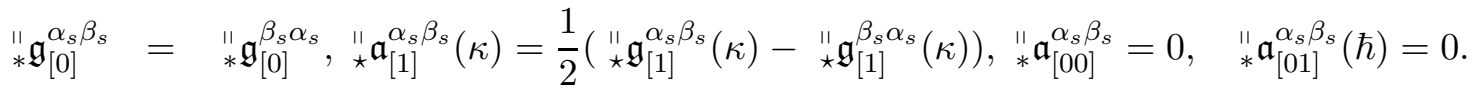

Any coefficient of above geometric s-objects can be also subjected to h1-v2-c3-c4 decompositions.

\subsubsection{Star deformed canonical s-adapted nonassociative vacuum gravitational equations}

In general, the canonical Ricci s-tensors are not symmetric for all (non) commutative and nonassociative cases. Contracting respectively with the symmetric and nonsymmetric components of a (star deformed) inverse s-metric, we define and compute the nonassociative nonholonomic canonical Ricci scalar curvature:

$$
\begin{aligned}
& { }_{s} \widehat{\mathbf{R}} s c^{\star}:={ }_{\star}{ }_{\star} \mathfrak{g}^{\mu_{s} \nu_{s}} " \widehat{\mathbf{R}} i c_{\mu_{s} \nu_{s}}=\left({ }_{\star}{ }_{\star} \mathfrak{g}^{\mu_{s} \nu_{s}}+{ }_{\star}{ }_{\star} \mathfrak{a}^{\mu_{s} \nu_{s}}\right)\left(" \widehat{\mathbf{R}} i c_{\left(\mu_{s} \nu_{s}\right)}+{ }^{"} \widehat{\mathbf{R}} i c_{\left[\mu_{s} \nu_{s}\right]}^{\star}\right)={ }_{s}{ }_{s} \widehat{\mathbf{R}} s s^{\star}+{ }_{s}{ }_{s} \widehat{\mathbf{R}} s a^{\star}, \\
& \text { where }{ }_{s} \widehat{\mathbf{R}} s s^{\star}=:{ }_{\star} \check{\mathfrak{g}}^{\mu_{s} \nu_{s}} " \widehat{\mathbf{R}} i c_{\left(\mu_{s} \nu_{s}\right)}^{\star} \text { and }{ }_{s} \widehat{\mathbf{R}} s a^{\star}:={ }_{\star} " \mathfrak{a}^{\mu_{s} \nu_{s}} " \widehat{\mathbf{R}} i c_{\left[\mu_{s} \nu_{s}\right]} \text {. }
\end{aligned}
$$

In this paper, respective symmetric (...) and anti-symmetric [...] decompositions are performed using respective symmetrization and anti-symmetrization with multiple $1 / 2$. For instance, for the second rank s-tensors, $" \widehat{\mathbf{R}} i c_{\mu_{s} \nu_{s}}^{\star}=" \widehat{\mathbf{R}} i c_{\left(\mu_{s} \nu_{s}\right)}^{\star}+" \widehat{\mathbf{R}} i c_{\left[\mu_{s} \nu_{s}\right]}^{\star}$.

Applying star nonholonomic s-deformations (51) to (26), we can define and compute s-adapted components of nonassociative vacuum gravitational equations,

$$
" \widehat{\mathbf{R}} i c_{\alpha_{s} \beta_{s}}^{\star}-\frac{1}{2}{ }_{\star} \mathfrak{g}_{\alpha_{s} \beta_{s}}{ }_{s}{ }_{s} \widehat{\mathbf{R}} s c^{\star}={ }_{s}{ }^{\prime \prime}{ }_{\star} " \mathfrak{g}_{\alpha_{s} \beta_{s}} .
$$

It is convenient to write down such systems of nonlinear PDEs in a form emphasizing explicitly the symmetric and nonsymmetric components of s-metric (54),

$$
\begin{aligned}
& { }_{\star}{ }_{\star} \check{\mathfrak{g}}_{\mu_{s} \nu_{s}}\left({ }_{s} \| \lambda+\frac{1}{2}{ }_{s}{ }_{s} \widehat{\mathbf{R}} s c^{\star}\right)=" \widehat{\mathbf{R}} i c_{\left(\mu_{s} \nu_{s}\right)}^{\star} \text { and } \\
& { }_{\star}^{\prime \prime} \mathfrak{a}_{\mu_{s} \nu_{s}}\left({ }_{s}{ }_{s} \lambda+\frac{1}{2}{ }_{s}{ }_{s} \widehat{\mathbf{R}} s c^{\star}\right)=" \widehat{\mathbf{R}} i c_{\left[\mu_{s} \nu_{s}\right]}^{\star} .
\end{aligned}
$$

Form the second system of equations, we observe that if we impose the nonholonomic conditions that

$$
\left({ }_{s}{ }_{s} \lambda+\frac{1}{2}{ }_{s}{ }_{s} \widehat{\mathbf{R}} s c^{\star}\right)=0 \text { and } " \widehat{\mathbf{R}} i c_{\left[\mu_{s} \nu_{s}\right]}^{\star}=0
$$


the s-adapted R-fluxes generate nonsymmetric components of s-metrics ${ }_{\star}^{\prime \prime} \mathfrak{a}_{\mu_{s} \nu_{s}}\left[\overline{\mathcal{R}}^{\tau_{s} \xi_{s}}{ }_{\beta_{s}} " \mathbf{e}_{\xi_{s}}{ }_{\star} \mathbf{g}_{\tau_{s} \alpha_{s}}\right]$ (洁3) for arbitrary symmetric solutions ${ }_{\star}^{\prime \prime} \check{g}_{\mu_{s} \nu_{s}}={ }_{\star}{ }_{\star} \mathfrak{g}_{\mu_{s} \nu_{s}}\left[{ }_{\star}{ }_{\star} \mathbf{g}_{\beta_{s} \tau_{s}}, \overline{\mathcal{R}}^{\tau_{s} \xi_{s}}{ }_{\beta_{s}} " \mathbf{e}_{\xi_{s}}{ }_{\star} \mathbf{g}_{\tau_{s} \alpha_{s}}\right]$ of (52) where coefficients are constructed as functionals. The symmetric part can taken to be a solution of

$$
" \widehat{\mathbf{R}} i c_{\mu_{s} \nu_{s}}^{\star}={ }_{s}^{\prime \prime} \lambda{ }_{\star}^{\|} \mathfrak{g}_{\alpha_{s} \beta_{s}},
$$

which is a star s-adapted deformation of (28). The symmetric solutions of such PDEs of zero order on parameters $\hbar$ and $\kappa$ can be used as prime ones for generating general (non) symmetric solutions with parametric dependence in nonassociative gravity and geometric flow theories, see next section.

For both type symmetric and nonsymmetric solutions of above systems of nonlinear PDEs for nonassociative vacuum gravity, we can extract LC-configurations imposing at the end certain nonholonomic conditions

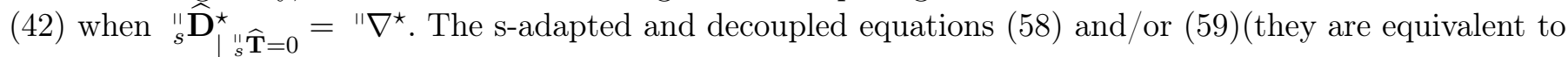
(61) ) can be re-written equivalently for " $\nabla^{\star}$ using canonical star distortions (41). For the LC-configurations, the Ricci tensor is always symmetric but in such holonomic variables we obtain vacuum gravitational equations written in "more coupled" nonlinear forms which can not be solved in some exact and well-defined parametric forms. Here we also emphasize that the systems of nonlinear PDEs with nonholonomic dyadic splitting of type (57) and, respectively, (58) and (59) etc. are self-consistent. Really, we can always start with a (off-) diagonal solution of commutative vacuum Einstein equations and compute s-adapted nonholonomic deforms and/or star deformations with respective term in nonassociative analogous of s-adapted star deformations, for a symmetric " $\mathbf{g}_{\alpha_{s} \beta_{s}}$. Of course, we can work with more general (non) associative configurations prescribing some commutative ansatz for ${ }_{\star}^{\prime \prime} \check{\mathfrak{g}}_{\alpha_{s} \beta_{s}}$ and ${ }_{\star}^{\prime \prime} \mathfrak{a}_{\mu_{s} \nu_{s}}$ (we can consider nonsymmetric canonical Ricci s-tensors) in a self-consistent form for a chosen s-adapted frame $" \mathbf{e}_{\mu_{s}}$ and then try to generate parametric solutions of vacuum nonassociative gravitational field equations with nontrivial $\mathrm{R}$-flux contributions. The main issue in such cases will be to keep such a s-adapted nonassociative gravitational dynamical / geometric evolution scenarios when certain decoupling of star deformed systems of PDEs will be possible.

There are nontrivial solutions of (58) and/or (59) for arbitrary parametric decomposition of ${ }_{\star} \mathfrak{a}_{\mu \nu}$ if

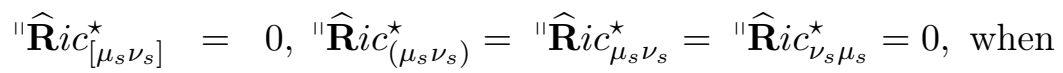

$$
\begin{aligned}
& { }_{s}{ }_{s} \widehat{\mathbf{R}} s c^{\star}={ }_{s}{ }_{s} \widehat{\mathbf{R}} s s^{\star}={ }_{\star} \check{\mathfrak{g}}^{\mu_{s} \nu_{s}} " \widehat{\mathbf{R}} i c_{\mu_{s} \nu_{s}}^{\star}={ }_{\star} \mathfrak{\mathfrak { g }}^{i_{1} j_{1}} " \widehat{\mathbf{R}} i c_{i_{1} j_{1}}^{\star}+{ }_{\star}{ }_{\star} \mathfrak{\mathfrak { g }}^{a_{2} b_{2}} " \widehat{\mathbf{R}} i c_{a_{2} b_{2}} \\
& +{ }_{\star} \check{\mathfrak{g}}_{a_{3} b_{3}} " \widehat{\mathbf{R}} i c^{\star a_{3} b_{3}}+{ }_{\star}{ }^{*} \check{\mathfrak{g}}_{a_{4} b_{4}} " \widehat{\mathbf{R}} i c^{\star a_{4} b_{4}}=0 .
\end{aligned}
$$

Such s-adapted star deformations with terms proportional up to $\hbar, \kappa$ and $\hbar \kappa$ can be considered for generating nonassociative generalizations of black hole solutions from GR. Such solutions will be constructed in partner works but some important and characteristic properties for stationary configurations will be analyzed in section 5.3 ,

In a more general context, we can compute nonzero " $\widehat{\mathbf{R}} s c^{\star}={ }^{1} \Lambda+{ }^{2} \Lambda+{ }^{3} \Lambda+{ }^{4} \Lambda$ for certain effective shell polarized cosmological constants ${ }^{s} \Lambda\left({ }_{s}^{\|} u\right)$ with certain dependence on shell coordinates. Such polarizations of a nonassociative gravitational vacuum determined by determined by nonlinear interactions in a nonassociative gravitational "eather" which is a star nonholonomic deformation of a vacuum configuration in GR. The nonassociative vacuum gravitational equations with polarized cosmological shell constants,

$$
\text { " } \widehat{\mathbf{R}} i c_{\alpha_{s} \beta_{s}}^{\star}\left(" u^{\gamma_{s}}\right)={ }^{s} \Lambda\left(" u^{\gamma_{s}}\right){ }_{\star} \check{\mathfrak{g}}_{\alpha_{s} \beta_{s}}\left(" u^{\gamma_{s}}\right),
$$

split in dyadic form for respective shells:

$$
\begin{aligned}
& \text { " } \widehat{\mathbf{R}} i c_{i_{1} j_{1}}^{\star}\left(x^{k_{1}}\right)={ }^{1} \Lambda\left(x^{k_{1}}\right){ }_{\star}{ }_{\star} \check{\mathfrak{g}}_{i_{1} j_{1}}\left(x^{k_{1}}\right), \\
& \text { " } \widehat{\mathbf{R}} i c_{a_{2} b_{2}}^{\star}\left(x^{k_{1}}, x^{c_{2}}\right)={ }^{2} \Lambda\left(x^{k_{1}}, x^{c_{2}}\right){ }_{\star}{ }_{\star} \check{\mathfrak{g}}_{a_{2} b_{2}}\left(x^{k_{1}}, x^{c_{2}}\right), \\
& \text { " } \widehat{\mathbf{R}} i c^{\star a_{3} b_{3}}\left(x^{k_{1}}, x^{c_{2}},{ }^{\prime \prime} p_{b_{3}}\right)={ }^{3} \Lambda\left(x^{k_{1}}, x^{c_{2}},{ }^{\prime \prime} p_{b_{3}}\right){ }_{\star}{ }_{\star} \check{\mathfrak{g}}^{a_{3} b_{3}}\left(x^{k_{1}}, x^{c_{2}}, " p_{b_{3}}\right),
\end{aligned}
$$

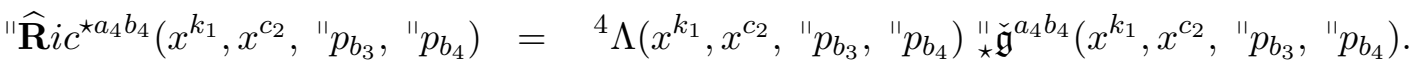


We can impose certain nonholonomic constraints

$$
{ }^{"} \lambda+\frac{1}{2} \text { " } \widehat{\mathbf{R}} s c^{\star}={ }^{"} \lambda+{ }^{1} \Lambda+{ }^{2} \Lambda+{ }^{3} \Lambda+{ }^{4} \Lambda=0,
$$

which allow to generate arbitrary nonsymmetric ${ }_{\star}^{\prime \prime} \mathfrak{a}_{\mu_{s} \nu_{s}}$ driven by a general off-diagonal symmetric and associative/ commutative s-metric " $\mathrm{g}_{\alpha_{s} \beta_{s}}$ and respective parametric R-flux deformations as in (52). Such nonholonomic constraints impose a nonholonomic dynamic/ geometric evolution in phase space for (non) symmetric metrics. In section 6, we shall integrate in general forms, recurrently, systems of nonlinear PDEs (64) with parametric s-decompositions of ${ }_{\star}^{\prime \prime} \mathfrak{g}_{\mu_{s} \nu_{s}}$ and ${ }_{\star}^{\|} \mathfrak{a}_{\mu_{s} \nu_{s}}$. We shall also define certain generalized nonassociative nonlinear symmetries which will allow to modify the generating functions and effective sources in such solutions to those determined with certain constant relations ${ }^{s} \Lambda\left({ }_{s} u\right) \rightarrow{ }_{0}^{s} \Lambda=$ const, in particular, with ${ }_{0}^{s} \Lambda={ }_{0}^{s} \Lambda=" \lambda / 4$.

\subsection{Vacuum gravitational equations with effective nonassociative s-sources}

Using parametric s-decompositions with ${ }_{\star}^{\|} \check{\mathfrak{g}}_{\alpha_{s} \beta_{s}}^{[0]}={ }_{\star}^{\|} \mathbf{g}_{\alpha_{s} \beta_{s}}(\underline{521})$ for the canonical Ricci s-tensor, we express the nonassociative vacuum gravitational field equations (63) in the form

$$
\begin{aligned}
& \text { " } \widehat{\mathbf{R}}_{\beta_{s} \gamma_{s}}={ }^{\prime} \mathbf{K}_{\beta_{s} \gamma_{s}} \text {, for effective nonassociative sources } \\
& { }^{"} \mathbf{K}_{\beta_{s} \gamma_{s}}={ }_{[0]}^{11} \Upsilon_{\beta_{s} \gamma_{s}}+{ }_{[1]}^{11} \mathbf{K}_{\beta_{s} \gamma_{s}}\lceil\hbar, \kappa\rceil \text {, where } \\
& { }_{[0]}{ }_{[0]} \Upsilon_{\beta_{s} \gamma_{s}}={ }^{s} \Lambda\left(" u^{\gamma_{s}}\right){ }_{\star}{ }_{\star} \mathbf{g}_{\beta_{s} \gamma_{s}} \text { and } \\
& { }_{[1]}^{\prime \prime} \mathbf{K}_{\beta_{s} \gamma_{s}}\lceil\hbar, \kappa\rceil={ }^{s} \Lambda\left(" u^{\gamma_{s}}\right){ }_{\star}{ }_{\star} \mathfrak{g}_{\beta_{s} \gamma_{s}}^{[1]}(\kappa)-" \widehat{\mathbf{K}}_{\beta_{s} \gamma_{s}}\lceil\hbar, \kappa\rceil
\end{aligned}
$$

is an effective parametric source with coefficients proportional to $\hbar, \kappa$ and $\hbar \kappa$.

In this work, we shall apply a procedure for decoupling and integrating nonassociative vacuum gravitational equations in parametric form when additional conditions of type (60) on (effective) cosmological constants and certain scalars can be always imposed. For such conditions, we shall be able to decouple the equations for the symmetric and nonsymmetric components of s-metrics. We shall begin with decoupling of the associative and commutative part of (66) with certain general parametrization of generating sources and finding certain general integrals for ${ }_{\star} \mathbf{g}_{\beta_{s} \gamma_{s} \mid \hbar, \kappa=0}={ }^{\prime \prime} \mathbf{g}_{\beta_{s} \gamma_{s}}$ following the results of our recent work [40], see also similar results in [21, 20, 19].

\subsubsection{Dyadic parametrization of effective nonassociatvie s-sources}

We are not able to decouple and integrate in certain general/ explicit forms modified (non) associative equations for general sources. There are necessary also a series of additional assumptions on parameterizations of nonassociative R-flux induced effective sources which allow to generate generic off-diagonal solutions in explicit forms. In this work, we shall parameterize (effective) sources (67) and (68) are parameterized in s-adapted diagonal form

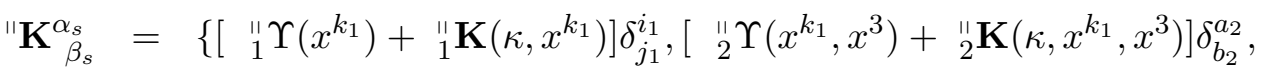

$$
\begin{aligned}
& \left.\left[{ }_{3}^{\prime \prime} \Upsilon\left(x^{k_{2}}, "{ }^{\prime \prime} p_{6}\right)+{ }_{3}{ }_{3} \mathbf{K}\left(\kappa, x^{k_{2}}, "{ }^{\prime \prime} p_{6}\right)\right] \delta_{b_{3}}^{a_{3}},\left[{ }_{4}{ }_{4} \Upsilon\left(x^{k_{3}}, "{ }^{\prime \prime} p_{8}\right)+{ }_{4}{ }_{4} \mathbf{K}\left(\kappa, x^{k_{3}}, "{ }^{\prime \prime} p_{8}\right)\right] \delta_{b_{4}}^{a_{4}}\right\}
\end{aligned}
$$

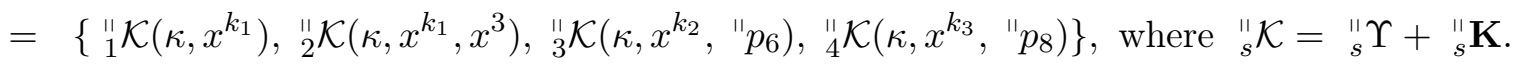

In these formulas, we consider a commutative spacetime background determined by ${ }^{\prime} \mathbf{g}_{\beta_{s} \gamma_{s}}$ (which can be used for transforming up/low indices into low/up ones up till orders $\kappa^{2}$ and $\hbar^{2}$ ) when the total spacetime geometry is defined by a parametric (non) symmetric s-metric. This will allow in next section to prove a parametric decoupling property of (66) for quasi-stationary (non) symmetric star metrics. In well defined dyadic frames 
the s-coefficients of such s-metrics do not depend on time like coordinated $x^{4}=t$ for the shells $s=1$ and 2 . We shall consider for phase s-metrics explicit dependencies on energy type coordinate " $p_{8} \sim E$.

A s-metric (and respective solutions of a (non) associative/ commutative ) gravitational equations is quasi-stationary if the corresponding (non) associative phase spacetime geometric s-objects possess a Killing symmetry on $\partial_{4}=\partial_{t}$ on shell $s=2$ and on " $\partial_{7}$, or " $\partial_{8}$, for all shells up to $s=4$. The AFCDM allows to construct very general classes of exact and parametric solutions for other conditions, for instance, with Killing symmetry $\partial_{3}$ on shell $s=2$ and on " $\partial_{8}$, or on " $\partial_{7}$, up to all shells $s=4$. The existence of such Killing vectors allows to integrate and find off-diagonal solutions in very general forms. In principle, we can construct more general classes of solutions without assumptions on existence of some Killing symmetries, see review of results and references in [4] but we do not present such cumbersome formulas in this paper 7

The nonassociative nonholonomic and parametric vacuum gravitational equations (66) with R-flux modified sources (69) can be written in dyadic form as

$$
\begin{aligned}
{ }^{"} \widehat{\mathbf{R}}_{\beta_{s}}^{\alpha_{s}} & ={ }^{\prime \prime} \mathbf{K}_{\beta_{s}}^{\alpha_{s}}[\hbar \kappa \overline{\mathcal{R}}], \text { where } \\
{ }^{"} \mathbf{K}_{\beta_{s}}^{\alpha_{s}} & =\left[{ }_{1}{ }_{1} \mathcal{K}\left(\kappa, x^{k_{1}}\right) \delta_{i_{1}}^{j_{1}},{ }_{2}{ }_{2} \mathcal{K}\left(\kappa, x^{k_{1}}, x^{3}\right) \delta_{b_{2}}^{a_{2}},{ }_{3}{ }_{3} \mathcal{K}\left(\kappa, x^{k_{2}}, "{ }^{\prime \prime} p_{6}\right) \delta_{a_{3}}^{b_{3}},{ }_{4}{ }_{4} \mathcal{K}\left(\kappa, x^{k_{3}}, "{ }^{1} p_{8}\right) \delta_{a_{4}}^{b_{4}}\right] .
\end{aligned}
$$

Such systems of nonlinear PDEs can be decoupled and solved in exact and parametric forms on 8-d phase spaces applying the AFCDM [40, 21] for nonholonomic $2+2+2+2$ splitting. In this work, we shall adapt the constructions to the case of momentum variables with complex unity and R-flux induced nonsymmetric and symmetric star deformed metrics.

Prescribing four couples of effective sources for certain diagonal data ${ }_{s}^{\prime \prime} \mathcal{K}=\left[{ }_{1}^{\prime \prime} \mathcal{K},{ }_{2} \mathcal{K} \mathcal{K},{ }_{3} \mathcal{K},,_{4} \mathcal{K}\right]$ in (70), we constrain nonholonomically shell by shell in dyadic adapted form the gravitational dynamics. For further constructions, we shall consider that we can prescribe any ${ }_{s} \mathcal{K}$ as generating sources encoding nonassociative geometric data. This will allow to express certain generic off-diagonal classes of exact/parametric solutions using some explicit formulas. We shall prove that such values and generating functions for sources are related to conventional cosmological constants via nonlinear symmetries, when the nonsymmetric parts of the smetrics and canonical Ricci s-tensors can be computed as R-flux deformations of some off-diagonal symmetric metric configurations.

\subsubsection{Computing parametric R-flux induced (non) symmetric metrics and effective source}

Considering an associative background solution ${ }_{\star}{ }_{\star} \mathbf{g}_{\beta_{s} \gamma_{s}} \simeq{ }^{\prime} \mathbf{g}_{\beta_{s} \gamma_{s}}$ of " $\widehat{\mathbf{R}}_{\beta_{s} \gamma_{s}}={ }_{[0]} \Upsilon_{\beta_{s} \gamma_{s}}$ from (66), we can compute the nonsymmetric s-adapted coefficients for s-metric (53) as induced ones by R-fluxes. The dyadic splitting is chosen with zero values of the commutative h1 and v2 components,

$$
\begin{aligned}
& { }_{\star}^{\prime \prime} \mathfrak{a}_{\alpha_{s} \beta_{s}}:=\quad\left[{ }_{\star}^{\prime \prime} \mathfrak{a}_{i_{1} j_{1}}=0, \quad{ }_{\star}^{\prime \prime} \mathfrak{a}_{a_{2} b_{2}}=0,\right. \\
& { }_{\star}^{\prime \prime} \mathfrak{a}^{a_{3} b_{3}}=\frac{i \kappa}{2}\left(\overline{\mathcal{R}}_{c_{3}}^{n+k_{s} a_{3}} "{ }^{\prime \prime} \mathbf{e}_{k_{s}} " \mathbf{g}^{c_{3} b_{3}}-\overline{\mathcal{R}}_{c_{3}}^{n+k_{s} b_{3}} "{ }^{\prime \prime} \mathbf{e}_{k_{s}} " \mathbf{g}^{c_{3} a_{3}}\right)={ }_{\star}{ }_{\star} \mathfrak{a}_{[1]}^{a_{3} b_{3}}(\kappa)=\frac{1}{2}\left({ }_{\star} \mathfrak{a}_{[1]}^{a_{3} b_{3}}-{ }_{\star} \mathfrak{g}_{[1]}^{b_{3} a_{3}}\right)(\kappa),
\end{aligned}
$$

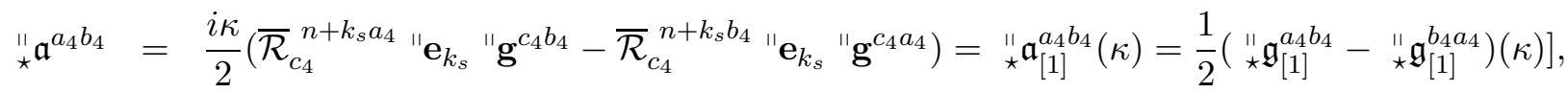

\footnotetext{
${ }^{7}$ One could be constructed also various examples of exact solutions, for instance, corresponding to triadic splitting $3+3+3+\ldots$, or a tetradic $4+4+\ldots$ one. Such higher dimension, $\geq 3$, shell by shell, decompositions can encode certain topology of respective dimension 3,4, etc. which may be different from the case of nonholonomic dyadic splitting. It is important to apply more advanced geometric and topologic methods to study properties of such physically important systems of nonlinear PDEs. One of the priorities of dyadic decompositions is that for 2-d shells we can always conventionally diagonalize block matrices which allow to decouple nonlinear equations in certain "convenient" systems of references/ coordinates. For such assumptions, we can construct solutions which depend, in principle, on all spacetime and phase space coordinates. Finally, we note that " $\mathcal{K}_{\beta_{s}}^{\alpha_{s}}$ can be related to a general non-diagonal source " $\widehat{\Upsilon}_{\alpha_{s}^{\prime} \beta_{s}^{\prime}}$ via frame transforms $" \widehat{\Upsilon}_{\alpha_{s}^{\prime} \beta_{s}^{\prime}}=e_{\alpha_{s}^{\prime}}^{\alpha_{s}} e_{\beta_{s}^{\prime}}^{\beta_{s}}$ " $\mathcal{K}_{\alpha_{s} \beta_{s}}$.
} 
where $i_{2}=\left(i_{1}, a_{2}\right)$, for $s=1,2$, following convention on s-adapted indices and coordinates. The symmetric part of s-metric is computed following parametric decompositions (52),

$$
\begin{aligned}
& " \check{\mathfrak{g}}_{\alpha_{s} \beta_{s}}=" \mathbf{g}_{\alpha_{s} \beta_{s}}+{ }_{\star} \check{\star}^{[} \mathfrak{g}_{\alpha_{s} \beta_{s}}^{[1]}(\kappa), \text { for } \\
& { }_{\star}^{\prime \prime} \mathfrak{\mathfrak { g }}_{\alpha_{s} \beta_{s}}^{[1]}(\kappa)=-\frac{i \kappa}{2}\left(\overline{\mathcal{R}}_{\beta_{s}}^{\tau_{s} \xi_{s}} " \mathbf{e}_{\xi_{s}}{ }^{\prime \prime} \mathbf{g}_{\tau_{s} \alpha_{s}}+\overline{\mathcal{R}}_{\alpha_{s}}^{\tau_{s} \xi_{s}} " \mathbf{e}_{\xi_{s}} "{ } \mathbf{g}_{\beta_{s} \tau_{s}}\right) \text {, }
\end{aligned}
$$

where $" \mathbf{g}_{\beta_{s} \tau_{s}}$ encode associative contributions from all shells $s=1,2,3,4$ but also result in symmetric R-flux deformations to star metrics.

So, prescribing any generating sources " $\mathbf{K}_{\beta_{s}}^{\alpha_{s}}$ (69) with nonassociative parametric deformations and using block $[(2 \times 2)+(2 \times 2)]+[(2 \times 2)+(2 \times 2)]$ parametrization of symmetric and nonsymmetric parts of star

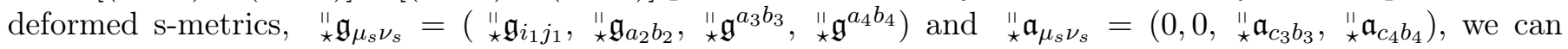
compute such R-flux deformations for any stated associative (off-) diagonal solution " $\mathbf{g}_{\alpha_{s} \beta_{s}}$. In a similar form, there are computed complex and real R-flux deformations of the canonical Ricci s-tensor (A.28), (A.29) and (A.31) subjected to nonholonomic constraints by generating sources in (49).

The main goal of this work is to extend the AFDM to nonassociative vacuum gravity described by dyadic equations (66) with effective sources (69) and prove a general decoupling property of such nonlinear systems of PDEs.

\subsection{Adapted lifts and horizontal projections of nonassociative canonical Ricci s-tensors}

On a Lorentz spacetime manifold $V$ and it (co) tangent bundle (and, for instance, on phase spaces $\mathcal{M}=T^{*} \mathbf{V}$ and/or " $\mathcal{M}$ ), we can define nonholonomic dyadic structures with shells $s=1,2,3,4$. Then, the geometric constructions can subjected to s-adapted nonassociative star deformations. Such deformations can be reordered using the associator and projected back to $\mathbf{V}$ in s-adapted form. Functions and s-forms can be lifted from $\mathbf{V}$ to $\mathcal{M}$ as pullbacks of s-forms using a respective s-adapted canonical projection $\pi: T^{*} \mathbf{V} \rightarrow \mathbf{V}$. To work in inverse direction, with an embedding $\sigma_{s}: \mathbf{V} \rightarrow \mathcal{M}$, which is given by zero section $x \rightarrow \sigma_{s}\left({ }^{1} x,{ }^{2} x\right)=\left({ }^{1} x,{ }^{2} x, 0,0\right)$, we pull back s-forms on $\mathcal{M}$ to s-forms on $\mathbf{V}$. Lifts of s-vectors are constructed using s-adapted foliations of $\mathcal{M}$ with constant momentum leaves with $2+2$ splitting. Such leafs are chosen to be diffeomorphic to $\mathbf{V}$ when for $\partial_{i_{2}}$ on $\mathbf{V}$ are transformed into a s-vector on $\mathcal{M}$, when

$$
\begin{aligned}
& b^{i_{2}}\left({ }^{1} x,{ }^{2} x\right) \partial_{i_{2}} \rightarrow \pi^{*}\left(b^{i_{2}}\right)\left({ }^{1} x,{ }^{2} x,{ }_{3}^{11} p,{ }_{4}^{11} p\right) \partial_{i_{2}} \text {, where } \\
& \pi^{*}\left(b^{i_{2}}\right)(x, p) \partial_{i_{2}}=\left(b^{i_{2}}\right)\left(\pi\left({ }^{1} x,{ }^{2} x,{ }_{3}{ }_{3} p,{ }_{4}^{11} p\right)\right)=b^{i_{2}}\left({ }^{1} x,{ }^{2} x\right) \text {. }
\end{aligned}
$$

Acting in such directions, we project s-vector fields on $\mathcal{M}$ into s-vector fields on $\mathbf{V}$ via zero section of $\sigma_{s}$, which can be written in s-adapted coordinate form,

$$
\begin{aligned}
& b^{i_{1}}\left({ }^{1} x,{ }^{2} x,{ }_{3}^{11} p, \quad{ }_{4}^{11} p\right) \partial_{i_{1}}+b^{a_{2}}\left({ }^{1} x,{ }^{2} x,{ }_{3}^{11} p, \quad{ }_{4}^{11} p\right) \partial_{a_{2}}+b_{a_{3}}\left({ }^{1} x,{ }^{2} x,{ }_{3}{ }_{3} p,{ }_{4}{ }_{4} p\right) \partial^{a_{3}}+b_{a_{4}}\left({ }^{1} x,{ }^{2} x,{ }_{3} p, \quad{ }_{4}{ }_{4} p\right) \partial^{a_{4}} \\
& \rightarrow b^{i_{1}}\left({ }^{1} x,{ }^{2} x, 0,0\right) \partial_{i_{1}}+b^{a_{2}}\left({ }^{1} x,{ }^{2} x, 0,0\right) \partial_{a_{2}} .
\end{aligned}
$$

To apply the AFCDM, we have to work on nonholonomic manifolds and (co) tangent Lorentz bundles with dyadic s-splitting and perform the constructions in s-adapted form, with formulas of type

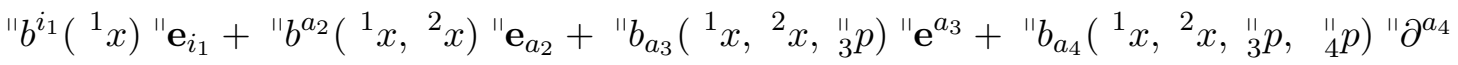

$$
\begin{aligned}
& \rightarrow \quad " b^{i_{1}}\left({ }^{1} x\right) " \mathbf{e}_{i_{1}}+{ }^{\prime \prime} b^{a_{2}}\left({ }^{1} x,{ }^{2} x\right) \text { " } \mathbf{e}_{a_{2}},
\end{aligned}
$$

see s-adapted bases (5). Any metric tensor $\widehat{g}_{j_{2} k_{2}}\left({ }^{1} x,{ }^{2} x\right) d x^{j_{2}} \otimes d x^{k_{2}}$ on $\mathbf{V}$, when $j_{2}=\left(j_{1}, b_{2}\right)$, can be lifted into an off-diagonal metric and/or equivalent s-metric ${ }_{s}^{\|} \mathbf{g}(19)$ on $\mathbf{T}_{s||}^{*} \mathbf{V}$. In s-adapted form, we can consider 
such shell by shell parameterizations using matrices (for symmetric s-tensors):

$$
\begin{aligned}
& \text { for } s=1,2 ; \text { for } \underline{\alpha}_{2} \underline{\beta}_{2}, \ldots, \text { or } i_{2}, j_{2}, \ldots=1,2,3,4 ;=\left(i_{1}, a_{2}\right),\left(j_{1}, b_{2}\right) \text {, for } i_{1}, j_{1}=1,2 ; a_{2}, b_{2}, \ldots=3,4 \text {; } \\
& \widehat{g}_{j_{1} k_{1}}\left({ }^{1} x\right) \rightarrow\left|\begin{array}{c}
" \widehat{g}_{\underline{\alpha}_{2} \underline{\beta}_{2}}\left(u^{\gamma_{2}}\right), \text { or } " \widehat{g}_{\underline{i}_{2} \underline{j}_{2}}\left(x^{k_{2}}\right), \\
\text { equivalent index } \\
\text { convention } \\
" \widehat{g}_{\underline{i}_{2} \underline{j}_{2}}\left(x^{k_{1}}, x^{c_{2}}\right)
\end{array}\right|= \\
& {\left[\begin{array}{cc}
" \widehat{g}_{i_{1} j_{1}}\left(x^{k_{1}}\right)+g_{a_{2} b_{2}}\left(x^{k_{2}}\right) N_{i_{1}}^{a_{2}}\left(x^{k_{2}}\right) N_{j_{1}}^{b_{2}}\left(x^{k_{2}}\right) & g_{a_{2} e_{2}}\left(x^{k_{2}}\right) N_{j_{1}}^{e_{2}}\left(x^{k_{2}}\right) \\
g_{b_{2} e_{2}}\left(x^{k_{2}}\right) N_{i_{1}}^{e_{2}}\left(x^{k_{2}}\right) & g_{a_{2} b_{2}}\left(x^{k_{2}}\right)
\end{array}\right]} \\
& \simeq\left[\widehat{\mathbf{g}}_{j_{1} k_{1}}\left(x^{k_{1}}\right), \mathbf{g}_{a_{2} b_{2}}\left(x^{k_{1}}, x^{c_{2}}\right)\right]={ }^{\prime} \mathbf{g}_{\mu_{2} \nu_{2}}\left(x^{k_{1}}, x^{c_{2}}\right)=\left[\begin{array}{cc}
\mathbf{g}_{j_{1} k_{1}}\left(x^{k_{1}}, x^{c_{2}}\right) & 0 \\
0 & \mathbf{g}_{a_{2} b_{2}}\left(x^{k_{1}}, x^{c_{2}}\right)
\end{array}\right] \\
& \longrightarrow \widehat{\mathbf{g}}_{j_{2} k_{2}}\left(x^{i_{2}}\right)=\left[\begin{array}{cc}
\mathbf{g}_{j_{1} k_{1}}\left(x^{k_{1}}, x^{c_{2}}\right) & 0 \\
0 & \quad \mathbf{g}_{a_{2} b_{2}}\left(x^{k_{1}}, x^{c_{2}}\right)
\end{array}\right] \text {, general s-coordinates ; }
\end{aligned}
$$

for $s=3 ;$ for $\underline{\alpha}_{3} \underline{\beta}_{3}, \ldots$, or $i_{3}, j_{3} \ldots=1,2,3,4,5,6 ;=\left(i_{2}, a_{3}\right),\left(j_{2}, b_{3}\right)$, for $a_{3}, b_{3}, \ldots=5,6$;

$$
\begin{aligned}
& \widehat{g}_{j_{2} k_{2}}\left(x^{i_{2}}\right) \rightarrow\left|\begin{array}{c}
" \widehat{g}_{\underline{\alpha}_{3}} \underline{\underline{\beta}}_{3}\left(" u^{\gamma_{3}}\right), \text { or } " \widehat{g}_{\underline{i}_{2} \underline{j}_{2}}\left(" x^{k_{3}}\right), \\
\text { equivalent index } \\
\text { convention } \\
" \widehat{g}_{\underline{i}_{3} \underline{j}_{3}}\left(x^{i_{2}}, " p_{a_{3}}\right)
\end{array}\right|=
\end{aligned}
$$

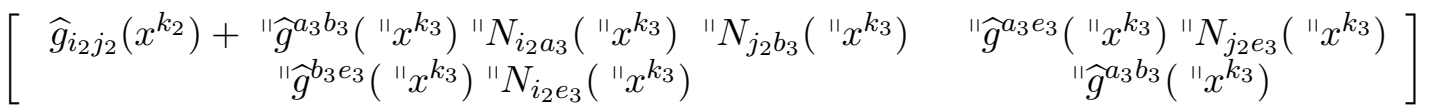

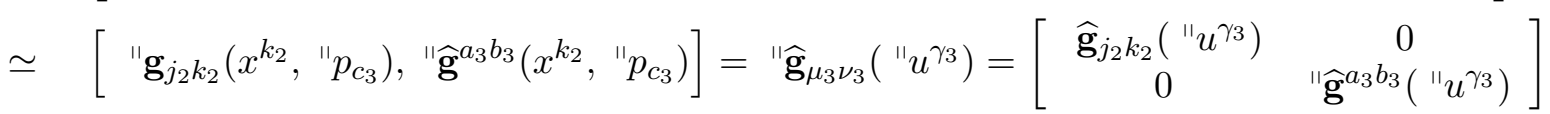

$$
\begin{aligned}
& \longrightarrow \quad " \widehat{\mathbf{g}}_{j_{3} k_{3}}\left({ }^{\prime \prime} x^{i_{3}}\right)=\left[\begin{array}{cc}
{ }^{"} \mathbf{g}_{j_{2} k_{2}}\left({ }^{"} x^{i_{3}}\right) & 0 \\
0 & " \mathbf{g}^{a_{3} b_{3}}\left(" x^{i_{3}}\right)
\end{array}\right]
\end{aligned}
$$

for $s=4 ;$ for $\underline{\alpha}_{4} \underline{\beta}_{4}, \ldots=1,2,3, \ldots 7,8 ;=\left(i_{3}, a_{4}\right),\left(j_{3}, b_{4}\right)$, for $a_{4}, b_{4}, \ldots=7,8$;

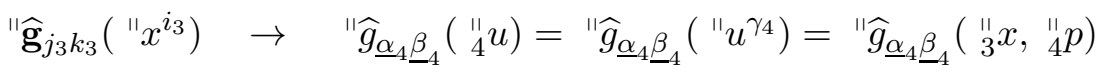

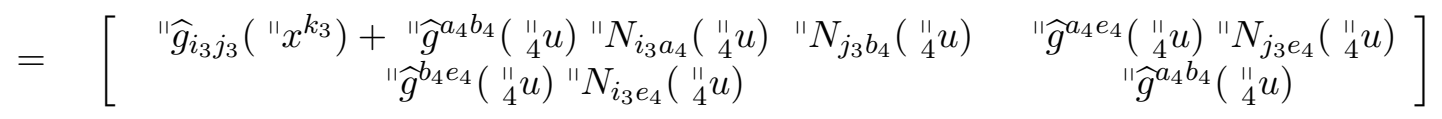

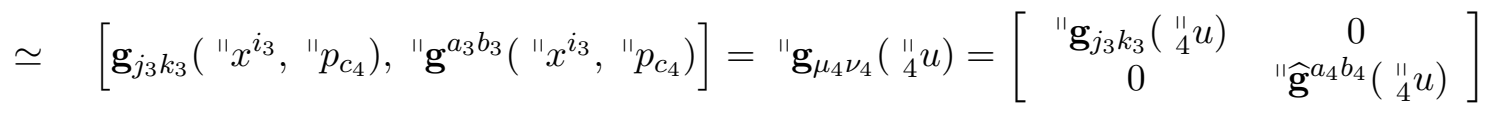$$
\longrightarrow \quad " \mathbf{g}_{\mu_{4} \nu_{4}}=\left[\begin{array}{cc}
\mathbf{g}_{j_{3} k_{3}}\left(" x^{i_{3}}, "{ }^{\prime \prime} p_{c_{4}}\right) & 0 \\
0 & " \mathbf{g}^{a_{4} b_{4}}\left(" x^{i_{3}}, "{ }^{\prime \prime} p_{c_{4}}\right)
\end{array}\right]
$$

In above formulas, there are used different variants of s-adapted notations in order to familiarize readers with such a symbolic nonholonomic and dyadic geometric calculus.

We can use an associative s-metric adapted in the form " $\mathbf{g}_{\alpha_{s} \beta_{s}}(73)$ instead of ${ }_{\star}\left(\mathbf{g}_{\alpha_{s} \beta_{s}}\right.$ and study s-adapted R-flux deformations to nonsymmetric s-metrics $" \mathrm{~g}_{\alpha \beta} \rightarrow{ }_{\star} " \mathfrak{g}_{\alpha \beta}$ (32), when

$$
{ }_{s}^{\prime \prime} \mathbf{g}=" \mathbf{g}_{\alpha_{s} \beta_{s}} " \mathbf{e}^{\alpha_{s}} \otimes_{\star s} " \mathbf{e}^{\beta_{s}} \rightarrow{ }_{\star} " \mathfrak{g}={ }_{\star} " \mathfrak{g}_{\alpha_{s} \beta_{s}} \star_{s}\left(" \mathbf{e}^{\alpha_{s}} \otimes_{\star s} " \mathbf{e}^{\beta_{s}}\right) .
$$

An important physical case is to consider R-deformations of s-metrics involving h1-v2-configurations determined by spacetime metrics, $\mathbf{g}_{i_{2} j_{2}}\left(x^{k_{2}}\right) \subset{ }_{\star} \mathfrak{g}_{\alpha_{s} \beta_{s}}$. For such nonassociative metric configurations, the linear 
R-flux corrections on shells $s=3,4$ can be parameterized in off-diagonal and/or dyadic block terms,

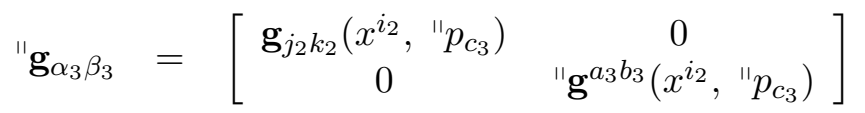

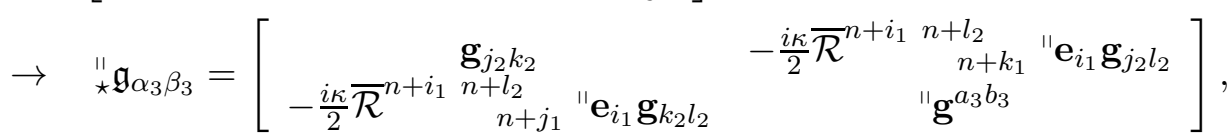

$$
\begin{aligned}
& \text { if } \mathbf{g}_{j_{2} k_{2}}=\mathbf{g}_{j_{2} k_{2}}\left(x^{i_{2}}\right) \rightarrow\left[\begin{array}{cc}
\mathbf{g}_{j_{2} k_{2}}\left(x^{i_{2}}\right) & -\frac{i \kappa}{2} \overline{\mathcal{R}}^{n+i_{1} n+l_{2}}{ }_{n+k_{1}} \partial_{i_{1}} \mathbf{g}_{j_{2} l_{2}} \\
-\frac{i \kappa}{2} \overline{\mathcal{R}}^{n+i_{1} n+l_{2}}{ }_{n+j_{1}} \partial_{i_{1}} \mathbf{g}_{k_{2} l_{2}} & \mathbf{g}^{a b_{3} b_{3}}
\end{array}\right] \text {; }
\end{aligned}
$$

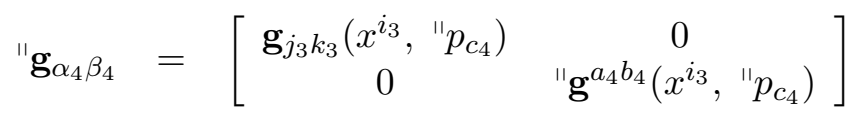

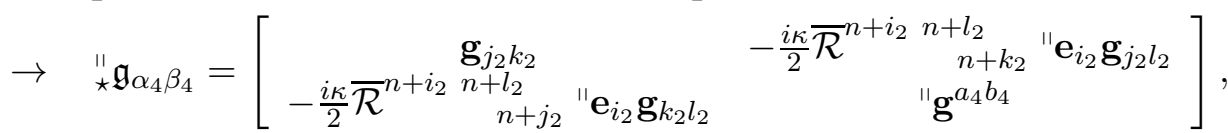

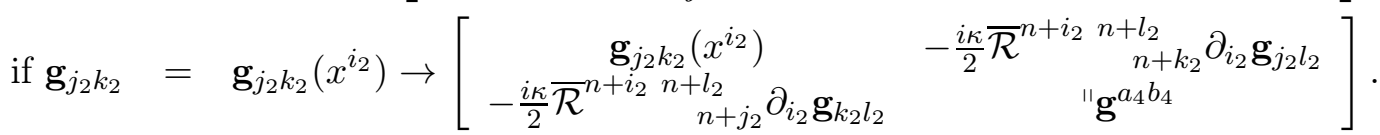

We note that in above formulas with nonassociative dyadic decompositions the R-flux terms mix and interrelate shells for a background dyadic splitting.

The h1-v2-projection of a Ricci s-tensor " $\widehat{\mathbf{R}} i c_{\alpha_{2} \beta_{2}}^{\star}=\left\{" \widehat{R} \star_{\star h_{2} j_{2}}\right\}$, see formulas (47), is a canonical sconnection analog of the nonassociative Ricci tensor for LC-configuration and projections on $V$. If we impose

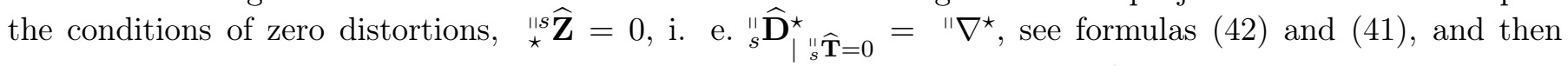
performing the constructions a corresponding local basis with dyadic splitting (then restricting the components of the Ricci tensors and s-tensors, $R i c_{\alpha_{s} \beta s}$, to spacetime directions and setting the momentum dependence to zero), we can construct geometric configurations with

$$
\text { " } \widehat{R}_{\star h_{2} j_{2}}\left({ }_{s} x,{ }_{s} " p\right) \rightarrow \quad{ }_{\nabla} \Re i c_{h_{s} j_{s}}^{\star}\left({ }_{s} x\right)=R i c_{h_{2} j_{2}}^{\circ}\left({ }_{s} x\right)=\sigma^{*}\left({ }_{\nabla} \Vdash R i c_{h_{2} j_{12}}^{\star}\left({ }_{s} x,{ }_{s}{ }_{s} p\right)\right)=R_{h j}\left({ }_{s} x, .\right) .
$$

In N-adapted form such nonassociative nonholonomic configurations and their spacetime projections were defined in section 4.4 of [3]. In this work, the dyadic formalism allows to generate such geometric models using s-metrics and star R-flux deformations via formulas (73) and (74) in order to show how such configurations can be extracted from solutions of nonassociative vacuum gravitational equations with parametric decompositions $\hbar$ and $\kappa$ (see, for instance, (62) ). Considering nonholonomic constraint to the case of LC-connection we obtain the nonassociative vacuum gravitational equations on phase space postulated in [2],

$$
{ }_{\nabla} \Re i c_{\alpha_{s} \beta_{s}}^{\star}\left({ }_{s} x,{ }_{s} p\right)=0 .
$$

For certain additional assumptions, every solution of such a system of nonlinear PDEs can be transformed as a solution of

$$
\operatorname{Ric}_{h_{2} j_{2}}^{\circ}\left(x^{k_{2}}\right)=0
$$

on the base spacteime $V$. In holonomic form, not all solutions of (76) can be lifted to solutions of (75) on the phase space because the "non-geometric" R-fluxes define nontrivial nonholonomic distributions both on the phase space and the spacetime manifold.

In a series of partner works (see [3] as the first one), we elaborate on geometrization of "non-geometric" string theories with R-fluxes using nonholonomic phase geometry. Following geometric principles similar to [24, 4, 5, 41] but for respective nonholonomic dyadic (non) symmetric and canonical s-connection structures, we can derive nonassociative dynamical gravitational and matter field equations and develop geometric flow evolution models completely determined on phase spaces. Such constructions can be completed with a geometric method of constructing exact and parametric solutions for nonassociative parametric physically important nonlinear systems of PDEs. 


\subsection{First order parametric nonassociative s-adapted spacetime corrections}

In this subsection, we analyze some important physical properties of h1-v2 components of nonassociative nonholonomic dyadic and parametric vacuum gravitational equations (66). Projecting such formulas on a base spacetime $V$, we can compute nontrivial real R-flux contributions. Such s-adapted configurations can be generated by effective sources prescribed only on shells $s=1$ and 2 , when $" \mathbf{K}_{\beta_{s}}^{\alpha_{s}}\left(x^{k_{2}}\right)$ (69) impose respective classes of nonholonomic constraints on nontrivial star distortions of the canonical Ricci s-tensors depending, in general, on all phase space coordinates but parameterized with generating sources defined below as in formulas (81), when ${ }_{s}^{\prime \prime} \mathcal{K}=\left[{ }_{1}^{\|} \mathcal{K}\left(x^{k_{1}}\right),{ }_{2}^{\|} \mathcal{K}\left(x^{k_{2}}\right)\right]$, for $s=1,2$, .

We consider a nontrivial dependence on momentum coordinates for the $s=1$ and 2 components of a s-metric $\mathbf{g}_{j_{2} k_{2}}\left({ }_{s} x,{ }_{s} p\right)$ (in particular, one can be fixed configurations with $\mathbf{g}_{j_{2} k_{2}}\left({ }_{s} x\right)$ ) for an arbitrary value of a c-component " $\mathrm{g}^{a_{s} b_{s}}\left({ }_{s} x,{ }_{s} p\right)$ in (74). Nonholonomic dyadic s-frame (8) and (9) can be prescribed with "small" canonical distortions (41) and (22) (we can consider general frame and coordinate transforms after the computations have been performed for such special nonholonomic configruations) when

$$
\begin{aligned}
& { }_{[00]}^{\|} \widehat{\boldsymbol{\Gamma}}_{j_{2} k_{2}}^{i_{2}}={ }^{\prime} \widehat{\boldsymbol{\Gamma}}_{j_{2} k_{2}}^{i_{2}}=\left(\widehat{L}_{j_{1} k_{1}}^{i_{1}}, \widehat{L}_{b_{2} k_{1}}^{a_{2}}, \widehat{C}_{j_{1} c_{2}}^{i_{1}}, \widehat{C}_{b_{2} c_{2}}^{a_{2}}\right) \text {; } \\
& { }_{[01]}^{\|} \widehat{\Gamma}_{j_{2} k_{2}}^{i_{2}}=0 \text {; } \\
& { }_{[10]}^{\|} \widehat{\boldsymbol{\Gamma}}_{j_{2} k_{2}}^{i_{2}}=-i \kappa \overline{\mathcal{R}}^{n+o_{2}} a_{s} n+l_{2} p_{a_{s}} " \mathbf{g}^{i_{2} q_{2}}\left(" \mathbf{e}_{l_{2}} " \mathbf{g}_{q_{2} r_{2}}\right)\left(" \mathbf{e}_{o_{2}} " \widehat{\boldsymbol{\Gamma}}_{j_{2} k_{2}}^{r_{2}}\right), \\
& { }_{[10]} \widehat{\boldsymbol{\Gamma}}_{j k}^{a_{s}}=-\frac{i \kappa}{2} \overline{\mathcal{R}}^{n+i_{2}} a_{s} n+q_{2} \| \mathbf{g}_{l_{2} q_{2}} " \mathbf{e}_{i_{2}} " \widehat{\boldsymbol{\Gamma}}_{j_{2} k_{2}}^{l_{2}}, \\
& { }_{[10]}^{\|} \widehat{\boldsymbol{\Gamma}}_{k_{2}}^{i_{2} a_{s}}=\frac{i \kappa}{2} \overline{\mathcal{R}}^{n+o_{2}} a_{s} n+l_{2} \quad " \mathbf{g}^{q_{2} i_{2}} " \mathbf{e}_{o_{2}}\left(" \mathbf{g}_{q_{2} r_{2}} " \widehat{\boldsymbol{\Gamma}}_{l_{2} k_{2}}^{r_{2}}\right), \\
& { }_{[10]}^{\|} \widehat{\boldsymbol{\Gamma}}_{k_{2}}^{i_{2} a_{s}}=\frac{i \kappa}{2} \overline{\mathcal{R}}^{n+o_{2} a_{s}} n+l_{s} \quad " \mathbf{g}^{i_{2} q_{2}} " \mathbf{e}_{o_{2}}\left(" \mathbf{g}_{q_{2} r_{2}} " \widehat{\boldsymbol{\Gamma}}_{l_{2} k_{2}}^{r_{2}}\right) ; \\
& { }_{[11]}^{\|} \widehat{\boldsymbol{\Gamma}}_{j_{2} k_{2}}^{i_{2}}=\frac{\hbar \kappa}{2} \overline{\mathcal{R}}^{n+o_{2}} n+q_{2} \quad n+l_{2}\left(" \mathbf{e}_{o_{2}} " \mathbf{g}^{i_{2} s_{2}}\right)\left(" \mathbf{e}_{q_{2}} " \mathbf{g}_{s_{2} r_{2}}\right)\left(" \mathbf{e}_{l_{2}} " \widehat{\boldsymbol{\Gamma}}_{j_{2} k_{2}}^{r_{2}}\right), \\
& \text { where, for instance, " } \mathbf{e}_{2}=\left\{\mathbf{e}_{l_{1}}, e^{a_{2}}\right\} \text {, "} \mathbf{g}_{q_{2} r_{2}}=\left\{g_{i_{1} q_{1}}, g^{a_{2} b_{2}}\right\}, " \mathbf{g}^{i_{2} q_{2}}=\left\{g^{i_{1} q_{1}}, g_{a_{2} b_{2}}\right\}, p_{a_{s}}=\left(p_{a_{3}}, p_{a_{4}}\right) \text {. }
\end{aligned}
$$

We have to consider respective formulas (22) and (37) for the canonical s-connections, their star deformations and parametric decompositions. Such s-coefficients result in nontrivial distortions from the LC-connection. This mean that for general nonholonomic distributions ${ }_{[01]}^{\prime \prime} \widehat{\boldsymbol{\Gamma}}_{j_{s} k_{s}}^{i_{s}}$ (A.24) and ${ }_{[01]}^{\prime \prime} \widehat{\mathbf{R}} i c_{\beta_{s} \gamma_{s}}^{\star}$ (A.28) being proportional to $\hbar$ may be not zero even respective values for the LC-connection are zero in certain coordinate frames, see formulas (5.82)-(5.85) in [2].

Let us consider how the nonholonomic dyadic splitting on h1-v2 components determine possible nontrivial spacetime R-flux contributions. We have such nontrivial imaginary [10] terms of the canonical Ricci s-tensor see in explicit computations for formulas (A.29), which for a corresponding s-adapting structure with "small" canonical distortions is computed an imaginary term

$$
\begin{aligned}
& { }_{[10]}^{\| \prime} \widehat{\mathbf{R}} i c_{j_{2} k_{2}}^{\star}=i \kappa \overline{\mathcal{R}}^{n+o a_{s}{ }^{n+l}} p_{a_{s}}\left\{-" \mathbf{e}_{i_{2}}\left[" \mathrm{~g}^{i_{2} q_{2}}\left(" \mathbf{e}_{l_{2}} \quad \mathrm{~g}_{q_{2} r_{2}}\right)\left(" \mathbf{e}_{o_{2}} " \widehat{\boldsymbol{\Gamma}}_{j_{2} k_{2}}^{r_{2}}\right)\right]\right. \\
& +{ }^{\prime \prime} \mathbf{e}_{k_{2}}\left[" \mathbf{g}^{i_{2} q_{2}}\left(" \mathbf{e}_{l_{2}} " \mathbf{g}_{q_{2} r_{2}}\right)\left(" \mathbf{e}_{o_{2}} " \widehat{\boldsymbol{\Gamma}}_{j_{2} i_{2}}^{r_{2}}\right)\right]
\end{aligned}
$$

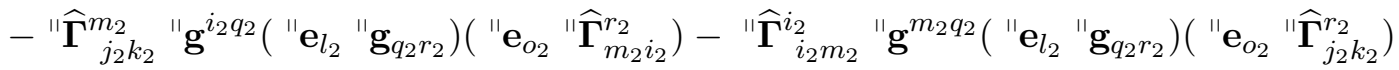

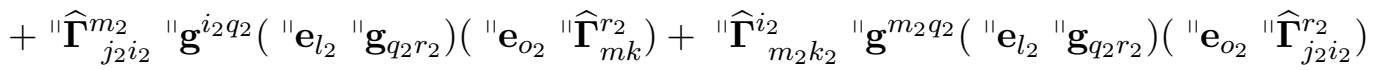

$$
\begin{aligned}
& \left.+\left({ }^{\prime \prime} \mathbf{e}_{l_{2}} " \widehat{\boldsymbol{\Gamma}}_{q_{2} i_{2}}^{i_{2}}\right)\left({ }^{\prime \prime} \mathbf{g}_{q_{2} r_{2}} " \widehat{\boldsymbol{\Gamma}}_{l_{2} k_{2}}^{r_{2}}\right)-\left({ }^{\prime} \mathbf{e}_{l_{2}} " \widehat{\boldsymbol{\Gamma}}_{q_{2} k_{2}}^{i_{2}}\right)\left({ }^{\prime \prime} \mathbf{g}_{q_{2} r_{2}} " \widehat{\boldsymbol{\Gamma}}_{l_{2} i_{2}}^{r_{2}}\right)\right\} \text {. }
\end{aligned}
$$


Nevertheless, there are also real nontrivial R-flux contributions:

$$
\begin{aligned}
& { }_{[11]}^{\|} \widehat{\mathbf{R}} i c_{j_{2} k_{2}}^{\star}=\frac{\hbar \kappa}{2} \overline{\mathcal{R}}^{n+o_{2}}{ }^{n+q_{2}}{ }^{n+l_{2}}\left\{" \mathbf{e}_{i_{2}}\left[\left(" \mathbf{e}_{o_{2}} " \mathbf{g}^{i_{2} m_{2}}\right)\left(" \mathbf{e}_{q_{2}} " \mathbf{g}_{m_{2} r_{2}}\right)\left(" \mathbf{e}_{l_{2}} " \widehat{\boldsymbol{\Gamma}}_{j_{2} k_{2}}^{r_{2}}\right)\right]\right. \\
& \text { - "} \mathbf{e}_{k_{2}}\left[\left(" \mathbf{e}_{O_{2}} " \mathbf{g}^{i_{2} m_{2}}\right)\left({ }^{"} \mathbf{e}_{q_{2}} " \mathbf{g}_{m_{2} r_{2}}\right)\left({ }^{"} \mathbf{e}_{l_{2}} " \widehat{\boldsymbol{\Gamma}}_{j_{2} i_{2}}^{r_{2}}\right)\right] \\
& +\left(" \mathbf{e}_{l_{2}} " \mathbf{g}_{q_{2} r_{2}}\right)\left["{ }^{\prime \prime} \mathbf{e}_{o_{2}}\left(" \mathbf{g}^{i_{2} q_{2}} " \widehat{\boldsymbol{\Gamma}}_{i_{2} k_{2}}^{m_{2}}\right)\left(" \mathbf{e}_{q_{2}} " \widehat{\boldsymbol{\Gamma}}_{j_{2} m_{2}}^{r_{2}}\right)-{ }^{\prime \prime} \mathbf{e}_{o_{2}}\left(" \mathbf{g}^{i_{2} q_{2}} " \widehat{\boldsymbol{\Gamma}}_{i_{2} m_{2}}^{m_{2}}\right)\left(" \mathbf{e}_{q_{2}} " \widehat{\boldsymbol{\Gamma}}_{j_{2} k_{2}}^{r_{2}}\right)\right. \\
& +\left(" \widehat{\boldsymbol{\Gamma}}_{j_{2} i_{2}}^{m_{2}} " \mathbf{e}_{o_{2}}\left({ }^{\prime} \mathbf{g}^{i_{2} r_{2}}\right)-" \mathbf{e}_{o_{2}}\left(" \widehat{\boldsymbol{\Gamma}}_{j_{2} i_{2}}^{m_{2}}\right) \quad \text { "g }{ }^{i_{2} r_{2}}\right)\left({ }^{"} \mathbf{e}_{q_{2}} " \widehat{\boldsymbol{\Gamma}}_{m_{2} k_{2}}^{r_{2}}\right) \\
& \left.\left.-\left(" \widehat{\boldsymbol{\Gamma}}_{j_{2} k_{2}}^{m_{2}} " \mathbf{e}_{o_{2}}\left(" \mathrm{~g}^{i_{2} r_{2}}\right)-" \mathbf{e}_{o_{2}}\left(" \widehat{\boldsymbol{\Gamma}}_{j_{2} k_{2}}^{m_{2}}\right) \quad " \mathbf{g}^{i_{2} r_{2}}\right)\left(" \mathbf{e}_{q_{2}} " \widehat{\boldsymbol{\Gamma}}_{m_{2} i_{2}}^{r_{2}}\right)\right]\right\} \text {. }
\end{aligned}
$$

Using the h1-v2 coefficients, we can write the respective s-coefficients of nonassociative vacuum Einstein equations (62) can be written in a form distinguishing real contributions from string gravity with R-fluxes,

$$
\text { " } \widehat{R}_{\star j_{2} k_{2}}\left({ }_{s} x,{ }_{s}{ }_{s} p\right)={ }^{\prime \prime} \widehat{R}_{j_{2} k_{2}}\left({ }_{s} x,{ }_{s}{ }_{s} p\right)+{ }_{[11]}^{\prime \prime} \widehat{\mathbf{R}} i c_{j_{2} k_{2}}^{\star}\left({ }_{s} x,{ }_{s}{ }_{s} p\right)=0 .
$$

Imposing additional nonholonomic constraints ${ }_{s}^{\prime \prime} \widehat{\mathbf{D}}_{\mid s}^{\star} \widehat{\mathbf{T}=0}={ }^{\prime \prime} \nabla^{\star}$ (42) for $s=1,2$, we extract from (178) horizontal LC-configurations of type (75),

$$
{ }_{\nabla}^{\|} \Re i c_{h_{2} j_{2}}^{\star}\left({ }_{s} x,{ }_{s}^{\prime \prime} p\right)={ }_{\nabla}^{\|} R R_{h_{2} j_{2}}\left({ }_{s} x,{ }_{s} p\right)+\underset{[11]}{\| \nabla} R_{h_{2} j_{2}}^{\star}\left({ }_{s} x,{ }_{s} p\right)=0 .
$$

If we work only with holonomic structures when $\mathbf{g}_{j_{2} k_{2}}\left({ }_{s} x,{ }_{s} p\right)=\mathbf{g}_{j_{2} k_{2}}\left({ }_{s} x\right)$, we generate on spacetime holonomic configurations of type (76),

$$
R i c_{h_{2} j_{2}}^{\circ}\left({ }_{s} x\right)=\nabla \operatorname{Ric}_{h_{2} j_{2}}\left({ }_{s} x\right)+{ }_{[11]}^{\|} R_{h_{2} j_{2}}^{\star}\left({ }_{s} x\right)=0 .
$$

In coordinate frames, these equations are equivalent to (5.90) from [2], when R-flux corrections to Ricci tensors determined on spacetime by $" \nabla$ are independent on $\hbar$. This property holds true for some special classes of metrics $\mathbf{g}_{j_{2} k_{2}}\left({ }_{s} x\right)$. Such a system of nonlinear PDEs can not decoupled and integrated in a general form when the coefficients of the s-metric are generic off-diagonal and depend on all spacetime coordinates with possible one Killing symmetry.

Applying the AFDM, we can decouple and integrate such equations in arbitrary form for various classes of smetrics $\mathbf{g}_{j_{2} k_{2}}\left({ }_{s} x,{ }_{s}{ }_{s} p\right) \rightarrow \mathbf{g}_{j_{2} k_{2}}\left({ }_{s} x\right)$ as solutions of (78). considered as a system of (66) with a nonholonomically constrained effective s-source " $\mathbf{K}_{\beta_{s}}^{\alpha_{s}}(\underline{69})$. We can search for nontrivial quasi-stationary configurations with

$$
\begin{aligned}
& { }^{"} \mathbf{K}_{\beta_{s}}^{\alpha_{s}}=\left\{{ }^{\prime \prime} \mathcal{K}_{j_{1}}^{i_{1}}=\left[{ }_{1}{ }_{1} \Upsilon\left(x^{k_{1}}\right)+{ }_{1}{ }_{1} \mathbf{K}\left(\kappa, x^{k_{1}}\right)\right] \delta_{j_{1}}^{i_{1}},\left\{{ }^{"} \mathcal{K}_{j_{2}}^{i_{2}}=\left[{ }_{2}{ }_{2} \Upsilon\left(x^{k_{1}}, x^{3}\right)+{ }_{2}{ }_{2} \mathbf{K}\left(\kappa, x^{k_{1}}, x^{3}\right)\right] \delta_{b_{2}}^{a_{2}},\right.\right. \\
& \text { where "K }{ }_{j_{2} k_{2}}=-{ }_{[11]} \widehat{\mathbf{R}} i c_{j_{2} k_{2}}^{\star}\left(x^{k_{1}}, x^{3}\right) \text { as in (77), } \\
& \text { for } \mathbf{g}_{j_{2} k_{2}}=\left\{g_{1}\left(x^{k_{1}}\right), g_{2}\left(x^{k_{1}}\right), g_{3}\left(x^{k_{1}}, x^{3}\right), g_{4}\left(x^{k_{1}}, x^{3}\right)\right\} \text {. }
\end{aligned}
$$

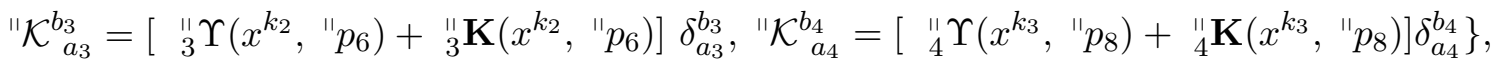

For such h1-v2 configurations, we can prescribe the effective sources (79) satisfying conditions of type (65)

$$
" \lambda+\frac{1}{2} " \widehat{\mathbf{R}} s c^{\star}=" \lambda+" \mathbf{K}_{\alpha_{s}}^{\alpha_{s}}[\hbar \kappa \overline{\mathcal{R}}]=0,
$$

where the contributions from shells $s=3,4$ are encoded into an effective cosmological constant " $\lambda$, but there are also nontrivial R-flux effective sources on shells $s=1,2$. We state such dependencies as functionals [...].

The system of real spacetime s-adapted projections of parametric nonassociative gravitational equations (66) with sources (79) transforms into

$$
\begin{aligned}
{ }^{"} \widehat{\mathbf{R}}_{i_{2} j_{2}}= & \quad{ } \mathbf{K}_{i_{2} j_{2}}[\hbar \kappa \overline{\mathcal{R}}], \text { where } \\
& \quad{ }^{j_{2}}=\left[{ }_{1}{ }_{i_{2}} \mathcal{K}\left(\kappa, x^{k_{1}}\right) \delta_{i_{1}}^{j_{1}},{ }_{2} \mathcal{K}\left(\kappa, x^{k_{1}}, x^{3}\right) \delta_{b_{2}}^{a_{2}}\right]
\end{aligned}
$$


The AFCDM method for constructing exact and parametric solutions of dimensions $2+2$ and with a number of examples of (off-) diagonal black hole and cosmological solutions is elaborated in [20], see references therein. Prescribing any generating sources ${ }_{s}^{\|} \mathcal{K}=\left[{ }_{1}^{\|} \mathcal{K},{ }_{2}^{\prime \prime} \mathcal{K}\right]$, for $s=1,2$, in (81), we can decouple and solve in certain general forms such systems of nonlinear PDEs.

So, we conclude that for certain classes on nonholonomic constraints on dyadic structure, the nonassociative vacuum gravitational equations on phase spaces can be transformed into self-consistent h1-v2 Einstein type equations for the canonical s-connection and with effective source from R-fluxes and nontrivial cosmological constant on certain shells. Any s-metric $\mathbf{g}_{j_{2} k_{2}}\left({ }_{s} x\right)$ solving (81) on a spacetime with R-flux effective sources defines on the total phase space respective nonsymmetric (71) and symmetric (72) star deformed s-metric structures. The s-adapted geometric objects on shells $s=1,2$ can be subjected to general s-frame spacetime transforms when, for instance, a parametric nonassociative and general non-diagonal source " $\widehat{\Upsilon}_{i_{2}^{\prime} j_{2}^{\prime}}=e_{i_{2}^{\prime}}^{i_{2}} e_{j_{2}^{\prime}}^{j_{2}} \quad$ " $\mathbf{K}_{i_{2} j_{2}}$ is defined.

\section{Decoupling \& integrability of nonassociative vacuum gravitational equa- tions}

In this section we extend and apply the anholonomic frame and connection deformation method, AFCDM, for constructing exact and parametric solutions in nonassociative gravity theories. In explicit form, our goal is to prove a general decoupling and integrability property of star deformed vacuum gravitational equations with parametric decomposition to systems of nonlinear PDEs on 8-d phase spaces, see (70), and/or 4-d spacetime systems (81), with R-flux corrections. In a rigorous nonholonomic dyadic geometric form, the AFCDM is elaborated for ral 8-d phase spaces modeled (co) tangent Lorentz bundles in Ref. [40] (see section 4 and respective appendices). It allows a trivial extension of such geometric methods and generated solutions for momentum like coordinates multiplied to the complex unity $i$. We shall omit proofs and cumbersome s-adapted computations because they are similar to those presented in a series of theorems of the just cited work but for a different type of effective sources, which in the nonassociative case contain contributions from R-fluxes and star nonholonomic products.

\subsection{Off-diagonal ansatz for phase space parametric s-metrics}

We consider dyadic decompositions when the total phase space s-metric structure may describe extensions of the BH stationary solutions in associative and commutative gravity to s-metrics with Killing symmetry on $\partial_{4}=\partial / \partial y^{4}=\partial_{t}=\partial / \partial t$ on the shell $s=2$; Killing symmetry " $\partial^{5}=\partial / \partial "{ }^{~} p_{5}$ on $s=3$, and posses a general and/or shell Killing symmetry on " $\partial^{7}=\partial / \partial " p_{7}$ on ${ }_{s} \mathbf{T}_{\|}^{*} \mathbf{V}$. The existence of such symmetries if very important for performing "simplified" proofs of decoupling property of modified Einstein equations in a number of (non) associative / commutative modified gravity theories. Such solutions allow us, for instance, to construct various extensions of the Schwarzschild and Kerr metrics in GR (with a prime diagonal metric $g_{i j}(r)=\operatorname{diag}\left[\ldots, \ldots,\left(1-r / r_{g}\right)^{-1},\left(1-r / r_{g}\right)\right]$. In this work, we shall use standard spacetime spherical coordinates with ' $\left.{ }^{\prime} u^{1}=x^{1}=r,{ }^{\prime} u^{2}=x^{2}=\theta,{ }^{\prime} u^{3}=y^{3}=\varphi,{ }^{\prime} u^{4}=y^{4}=t\right)$ when the phase space solutions depend in explicit form on the energy type variable " $p_{8}=" E$ and " $p_{6}$, (also on " $p_{5}$ via $\mathrm{N}$-connection coefficients) but not on " $p_{7}$. It is supposed that the coefficients of s-metrics are such way parameterized that possible terms with complex unity before coordinates are such way multiplied to some coefficients with complex unity when the resulting s-adapted coefficients are real. In certain cases, we shall use real phase coordinates like ${ }^{\prime} p_{8}=E$ and ' $p_{6}$, etc. We shall construct and study explicit examples of nonassociative of $\mathrm{BH}$ hole configurations gravity in our partner works. Such solutions are similar to those provided in [21]. 


\subsubsection{Quasi-stationary ansatz for s-metrics and R-flux sources}

For an associative and commutative s-metric ${ }_{s}^{\|} \mathbf{g}={ }^{\prime \prime} \mathbf{g}_{\alpha_{s} \beta_{s}}\left({ }_{s} x,{ }_{s} p\right) " \mathbf{e}^{\alpha_{s}} \otimes{ }^{\prime} \mathbf{e}^{\beta_{s}}$ (19), we consider a quasi-stationary ansatz for a linear quadratic element with additional parametric dependence on $\hbar$ and $\kappa$ of coefficients (which will be computed in next sections),

$$
\begin{aligned}
& d^{\prime \prime} \widehat{s}^{2}=\widehat{g}_{1}(r, \theta) d r^{2}+\widehat{g}_{2}(r, \theta) d \theta^{2}+\widehat{g}_{3}(r, \theta, \varphi) \delta \varphi^{2}+\widehat{g}_{4}(r, \theta, \varphi) \delta t^{2}+" \widehat{g}^{5}\left(r, \theta, \varphi, t, " p_{6}\right)\left(" \widehat{\mathbf{e}}_{5}\right)^{2}+ \\
& " \widehat{g}^{6}\left(r, \theta, \varphi, t, " p_{6}\right)\left(" \widehat{\mathbf{e}}_{6}\right)+{ }^{\prime \prime} \widehat{g}^{7}\left(r, \theta, \varphi, t, " p_{6}, " E\right)\left(" \widehat{\mathbf{e}}_{7}\right)^{2}+" \widehat{g}^{8}\left(r, \theta, \varphi, t, " p_{6}, "{ }^{\prime} E\right)\left(" \widehat{\mathbf{e}}_{8}\right)^{2} \text {, }
\end{aligned}
$$

where the s-adapted coefficients for the phase metric are chosen in the form

$$
\begin{aligned}
\widehat{g}_{i_{1} j_{i}} & =\operatorname{diag}\left[\widehat{g}_{i_{1}}\left(x^{k_{1}}\right)\right], \text { for } i_{1}, j_{1}=1,2 \text { and } x^{k_{1}}=\left(x^{1}=r, x^{2}=\theta\right) ; \\
\widehat{g}_{a_{2} b_{2}} & =\operatorname{diag}\left[\widehat{g}_{a_{2}}\left(x^{k_{1}}, y^{3}\right)\right], \text { for } a_{2}, b_{2}=3,4 \text { and } y^{3}=x^{3}=\varphi, y^{4}=x^{4}=t ; \\
"{ }^{a_{3} b_{3}} & =\operatorname{diag}\left["{ }^{a_{3}}\left(x^{k_{1}}, y^{a_{2}}, " p_{6}\right)\right], \text { for } a_{3}, b_{3}=5,6 \text { and " } u^{5}=" p_{5}, \quad " u^{6}=" p_{6} ; \\
" \widehat{g}^{a_{4} b_{4}} & =\operatorname{diag}\left["{ }^{a_{4}}\left(x^{k_{1}}, y^{a_{2}}, " p_{a_{3}}, " E\right)\right], \text { for } a_{4}, b_{4}=7,8 \text { and " } u^{7}=" p_{7}, \quad " u^{8}=" p_{8}=" E .
\end{aligned}
$$

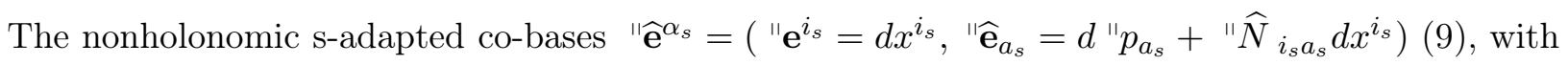

$$
\begin{aligned}
& e^{1}=d r, e^{2}=d \theta, \widehat{\mathbf{e}}^{3}=\delta \varphi=d \varphi+w_{i_{1}} d x^{i_{1}}, \widehat{\mathbf{e}}^{4}=\delta t=d t+n_{i_{1}} d x^{i_{1}}, \\
& \text { " } \widehat{\mathbf{e}}_{5}=d " p_{5}+" n_{j_{2}} d x^{j_{2}}, " \widehat{\mathbf{e}}_{5}=d " p_{5}+" n_{j_{2}} d x^{j_{2}},
\end{aligned}
$$

are determined by N-connection s-adapted coefficients

$$
\begin{aligned}
& \text { " } \widehat{N}_{j_{1}}^{3}={ }_{2} w_{j_{1}}=w_{j_{1}}(r, \theta, \varphi), \quad " N_{j_{1}}^{4}={ }_{2} n_{j_{1}}=n_{j_{1}}(r, \theta, \varphi) \\
& \text { " } \widehat{N}_{j_{2} 5}={ }_{3}^{"} n_{j_{2}}\left(x^{k_{2}}, " p_{6}\right)=" n_{j_{2}}\left(r, \theta, \varphi, t, " p_{6}\right) \text {, } \\
& \text { " } \widehat{N}_{j_{2} 6}={ }_{3}^{" 1} w_{j_{2}}\left(x^{k_{2}}, " p_{6}\right)=" w_{j_{2}}\left(r, \theta, \varphi, t, " p_{6}\right) \text { for } j_{2}=1,2,3,4 \text {; } \\
& \text { " } \widehat{N}_{j_{3} 7}={ }_{4} n_{j_{3}}\left(x^{k_{3}}, "{ }^{\prime} p_{8}\right)={ }^{"} n_{j_{3}}\left(r, \theta, \varphi, t, "{ }^{\prime} p_{6}, " E\right), \\
& \text { " } \widehat{N}_{j_{3} 8}={ }_{4}{ }_{4} w_{j_{3}}\left(x^{k_{3}},{ }^{\prime \prime} p_{6}, "{ }^{\prime \prime} p_{8}\right)={ }^{\prime \prime} w_{j_{3}}\left(r, \theta, \varphi, t, " p_{6}, "{ }^{\prime \prime} E\right) \text { for } j_{3}=1,2,3,4,5,6 \text {. }
\end{aligned}
$$

In above formulas, we put "hat" labels on s-metric and N-connection coefficients for a stationary ansatz which will be used for generating exact and parametric solutions of some modified (non) associative / commutative Einstein equations for a respective canonical s-connection ${ }_{s} \widehat{\mathbf{D}}$ (21) with s-adapted coefficients (22).

In this paper, the term "stationary" is used for metrics on phase space and base spacetime manifolds, which in certain special coordinates do not depend on respective time like coordinates but contain some off-diagonal terms. In GR, such terms describe, for instance, rotating Kerr BH, see [25, 24, 26, 27] but in our approach we work with general nonholonomic configurations (not only with coordinate rotating frames). The h1-v2 part (i.e. the first 4 terms for a Lorentz manifold base) in (82) is of stationary type. Nevertheless, the cofiber part (next 5-8 terms) of that ansatz may depend on a time like coordinate " $t$ ". This is because various modified dispersion relations and R-flux sources induce, in general, a nonholonomic cofiber dynamics with dependence on "t", and on "E" (in literature, it is used the term "rainbow" metrics for a dependence on a E-parameter). We use the term "quasi-stationary" for s-metric ansatz because the associated N-connection s-structure is nonholonomic and depends in local anisotropic form on phase space coordinates. Such ansatz allows us to extend the AFCDM to R-flux sources when a corresponding nonholonomic shell dyadic decompositions allows us to construct exact/parameteric quasi-stationary solutions as we shall prove in section 5.3. Here we note that an ansatz (82) is stationary if the s-metric and N-connection coefficients are prescribed in such an adapted form that the geometric s-objects do not depend explicitly on the time like variable $t$. In dual form, such ansatz and corresponding geometric methods of constructing exact solutions can be re-defined when the $t$-dependence is important for constructing locally anisotropic cosmological solutions, see details in [20]. 
We shall be able to generate in explicit form exact off-diagonal solutions of nonassociative modified vacuum Einstein equations on phase space for effective sources " $\mathbf{K}_{\beta_{s}}^{\alpha_{s}}=\delta_{\beta_{s}}^{\alpha_{s}}{ }_{s}^{\prime \prime} \mathcal{K}$, for $s=1,2,3,4$ in (70), or for respective real R-flux spacetime contributions (77) in " $\mathbf{K}_{j_{2}}^{i_{2}}=\delta_{j_{2}}^{i_{2}}{ }_{s}^{\prime \prime} \mathcal{K}$ for (81), for $s=1,2$. Those parametrization of (effective) nonassociative and associative source were stated in a quasi-stationary form. In explicit form, ${ }_{s} \mathcal{K}$ should be considered as values determined by certain phenomenological/ experimental/observational data or computed nonassociative sectors of string theory.

\subsubsection{Nonassociative and parametric canonical Ricci s-tensors}

In this work, there are used also short notations for partial derivatives when, for instance, $\partial_{1} q=q^{\bullet}, \partial_{2} q=$ $q^{\prime}, \partial_{3} q=\partial_{\varphi} q=q^{\diamond}, " \partial^{6} q=\partial q / \partial " p^{6}$, and $" \partial^{8} q=\partial q / \partial " p^{8}=\partial q / \partial "{ }^{\prime \prime}={ }^{\prime \prime} \partial_{E} q=q^{\prime \prime *}$ (we write " $A^{*}$ if a letter/formula has already a left label, " $A$ ). If in some formulas, the momentum like coordinates will be considered without complex unity, we shall write, respectively, ${ }^{\prime} \partial^{6} q=\partial q / \partial{ }^{\prime} p^{6}$, and ${ }^{\prime} \partial^{8} q=\partial q / \partial{ }^{\prime} p^{8}=$ $\partial q / \partial E={ }^{\prime} \partial_{E} q=q^{*}$.

For quasi-stationary configurations, we can always define s-adapted frame and coordinate transforms in ansatz (82) with $g_{4}^{\diamond} \neq 0, " \partial^{6} " g^{5} \neq 0$ and $\left(" g^{7}\right)^{\prime \prime *} \neq 0$. If such conditions are note imposed, we can construct more special classes of exact and parametric solutions but the formulas are more cumbersome and may not allow an explicit integration of motion equations. A tedious computation of the coefficients of the canonical s-connection and respective canonical Ricci s-tensor (24) of ${ }_{s}^{\prime \prime} \widehat{\mathbf{D}}(\underline{22})$ for a s-metric (82), see details on proof of Lemma 4.1 in [40] allows to write the system of vacuum s-adapted gravitational equations (170) with R-flux effective sources ${ }_{s} \mathcal{K}$ in the form

$$
\begin{aligned}
\widehat{R}_{1}^{1} & =\widehat{R}_{2}^{2}=\frac{1}{2 g_{1} g_{2}}\left[\frac{g_{1}^{\bullet} g_{2}^{\bullet}}{2 g_{1}}+\frac{\left(g_{2}^{\bullet}\right)^{2}}{2 g_{2}}-g_{2}^{\bullet \bullet}+\frac{g_{1}^{\prime} g_{2}^{\prime}}{2 g_{2}}+\frac{\left(g_{1}^{\prime}\right)^{2}}{2 g_{1}}-g_{1}^{\prime \prime}\right]=-{ }_{1} \mathcal{K}(\kappa, r, \theta), \\
\widehat{R}_{3}^{3} & =\widehat{R}_{4}^{4}=\frac{1}{2 g_{3} g_{4}}\left[\frac{\left(g_{4}^{\diamond}\right)^{2}}{2 g_{4}}+\frac{g_{3}^{\diamond} g_{4}^{\diamond}}{2 g_{3}}-g_{4}^{\diamond \diamond}\right]=-{ }_{2}^{\prime \prime} \mathcal{K}(\kappa, r, \theta, \varphi), \\
\widehat{R}_{3 k_{1}} & =\frac{w_{k_{1}}}{2 g_{4}}\left[g_{4}^{\diamond}-\frac{\left(g_{4}^{\diamond}\right)^{2}}{2 g_{4}}-\frac{\left(g_{3}^{\diamond}\right)\left(g_{4}^{\diamond}\right)}{2 g_{3}}\right]+\frac{g_{4}^{\diamond}}{4 g_{4}}\left(\frac{\partial_{k_{1}} g_{3}}{g_{3}}+\frac{\partial_{k_{1}} g_{4}}{g_{4}}\right)-\frac{\partial_{k_{1}}\left(g_{3}^{\diamond}\right)}{2 g_{3}}=0 ; \\
\widehat{R}_{4 k_{1}} & =\frac{g_{4}}{2 g_{3}} n_{k_{1}}^{\diamond}+\left(\frac{3}{2} g_{4}^{\diamond}-\frac{g_{4}}{g_{3}} g_{3}^{\diamond}\right) \frac{n_{k_{1}}^{\diamond}}{2 g_{3}}=0,
\end{aligned}
$$

on shells $s=1$ and $s=2$, with $i_{1}, k_{1} \ldots=1,2$;

$$
\begin{aligned}
& \text { " } \widehat{R}_{5}^{5}={ }^{\prime \prime} \widehat{R}_{6}^{6}=-\frac{1}{2 " g^{5} " g^{6}}\left[\frac{\left(" \partial^{6}\left(" g^{5}\right)\right)^{2}}{2 " g^{5}}+\frac{\left(" \partial^{6}\left(" g^{5}\right)\right)\left(" \partial^{6}\left(" g^{6}\right)\right)}{2 " g^{6}}-" \partial^{6} " \partial^{6}\left(" g^{5}\right)\right]= \\
& -{ }_{3} \mathcal{K}\left(\kappa, r, \theta, \varphi, t, " p_{6}\right) \text {, } \\
& " \widehat{R}_{5 k_{2}}=\frac{" g^{5}}{2 " g^{6}} " \partial^{6} " \partial^{6} " n_{k_{2}}+\left(\frac{3}{2} " \partial^{6} " g^{5}-\frac{" g^{5}}{" g^{6}} " \partial^{6} " g^{6}\right) \frac{" \partial^{6} " n_{k_{2}}}{2 " g^{6}}=0, \\
& { }^{"} \widehat{R}_{6 k_{2}}=\frac{" w_{k_{2}}}{2 "{ }^{5}}\left[" \partial^{6} " \partial^{6}\left(" g^{5}\right)-\frac{\left(" \partial^{6} " g^{5}\right)^{2}}{2 " g^{5}}-\frac{\left(" \partial^{6}\left(" g^{5}\right)\right)\left(" \partial^{6}\left(" g^{6}\right)\right)}{2 " g^{6}}\right] \\
& +\frac{" \partial^{6}\left(" g^{5}\right)}{4 " g^{6}}\left(\frac{{ }^{\prime \prime} \partial_{2} " g^{5}}{" g^{5}}+\frac{\partial_{k_{2}} " g^{6}}{" g^{6}}\right)-\frac{\partial_{k_{2}} " \partial^{6}\left(" g^{6}\right)}{2 " g^{6}}=0 ;
\end{aligned}
$$


on shell $s=3$ with $i_{2}, k_{2} \ldots=1,2,3,4$;

$$
\begin{aligned}
& \text { " } \widehat{R}_{7}^{7}=\quad " \widehat{R}_{8}^{8}=-\frac{1}{2 " g^{7} " g^{8}}\left[\frac{\left(\left(" g^{7}\right)^{*}\right)^{2}}{2 " g^{7}}+\frac{\left(" g^{7}\right)^{*}\left(" g^{8}\right)^{*}}{2 " g^{8}}-\left(" g^{7}\right)^{* *}\right]= \\
& -{ }_{4} \mathrm{~K}\left(\kappa, r, \theta, \varphi, t, " p_{5}, " p_{6}, " \mathrm{E}\right), \\
& { }^{\prime \prime} \widehat{R}_{7 k_{3}}=\frac{{ }^{\prime \prime} g^{7}}{2 " g^{8}} n_{k_{3}}^{* *}+\left(\frac{3}{2}\left({ }^{"} g^{7}\right)^{*}-\frac{" g^{7}}{" g^{8}}\left(" g^{8}\right)^{*}\right) \frac{{ }^{"} n_{k_{3}}^{*}}{2{ }^{"} g^{8}}=0, \\
& " \widehat{R}_{8 k_{2}}=\frac{" w_{k_{3}}}{2{ }^{"} g^{7}}\left[\left(" g^{7}\right)^{* *}-\frac{\left(\left(" g^{7}\right)^{*}\right)^{2}}{2 " g^{7}}-\frac{\left(" g^{7}\right)^{*}\left(" g^{8}\right)^{*}}{2 " g^{8}}\right]+\frac{\left(" g^{7}\right)^{*}}{4 " g^{8}}\left(\frac{\partial_{k_{3}} " g^{7}}{" g^{7}}+\frac{\partial_{k_{3}} " g^{8}}{" g^{8}}\right)-\frac{\partial_{k_{3}}\left(" g^{8}\right)^{*}}{2 " g^{8}}=0
\end{aligned}
$$

on shell $s=4$ with $i_{3}, k_{3} \ldots=1,2,3,4,5,6$.

Using the above formulas, we can compute the Ricci scalar (25) for ${ }_{s} \widehat{\mathbf{D}}(22)$, and find such relations for shell components of quasi-stationary phase spaces,

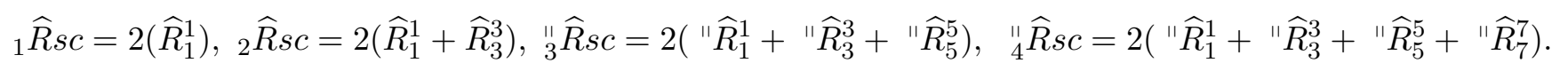

Such formulas define additional s-adapted symmetries the Einstein canonical s-tensor (26) of the quasistationary phase spaces and their parametric R-flux deformations. This allows to write the nonassociative vacuum gravitational equations (70) in the form

$$
\begin{aligned}
\widehat{R}_{1}^{1} & =\widehat{R}_{2}^{2}=-{ }_{1}{ }_{1} \mathcal{K} ; \widehat{R}_{3}^{3}=\widehat{R}_{4}^{4}=-{ }_{2}{ }_{2} \mathcal{K} ; \\
{ }^{"} \widehat{R}_{5}^{5} & ={ }^{\prime} \widehat{R}_{6}^{6}=-{ }_{3}{ }_{3} \mathcal{K} ; \quad "{ }^{2} \widehat{R}_{7}^{7}="{ }^{2} \widehat{R}_{8}^{8}=-{ }_{4}{ }_{4} \mathcal{K} .
\end{aligned}
$$

If the (effective) sources on shells $s=3,4$ are prescribed using formulas (67) and (68) for respective parameterizations that ${ }_{3}^{11} \mathcal{K}={ }_{4}^{11} \mathcal{K}=0$, we can consider trivial solutions for (87) on the cofibers of phase spaces. In result the nonlinear system (70) reduces to (86) in a form equivalent to (81) and (83). In result, we obtain a decoupling of respective system of nonlinear PDEs for a quasi-stationary nonholonomic 4-d spacetime involving parametric R-flux contributions (77) into the effective source " $\mathbf{K}_{j_{2}}^{i_{2}}=\delta_{j_{2}}^{i_{2}}{ }_{s} \mathcal{K}$, for $s=1,2$. We study the decoupling property of such phase and spacetime systems in details in next subsection.

\subsection{Decoupling of nonassociative nonholonomic stationary vacuum Einstein equations}

We can apply the Theorem 4.1 from [40] if we redefine those geometric constructions for phase spaces with complex momenta variables and effective sources involving parametric R-flux contributions. The priority of the AFDCM is that for various generalizations and modifications we can take certain known geometric results for nonlinear systems of PDEs (with a rigorous proof in previous works) and apply a symbolic geometric calculus when by analogy left labels change ${ }_{s} \rightarrow{ }_{3}^{\prime \prime}$ and associative sources change into nonassociative parametric ones, ${ }_{s} \widehat{\Upsilon} \rightarrow{ }_{s}^{\prime \prime} \mathcal{K}$.

The nonassociative vacuum gravitational field equations (70) (represented in the form (86) and (87) with canonical Ricci s-tensors (83), (84) and (85), computed for a quasi-stationary s-metric ansatz (82)) decouple on respective shells of phase space:

$$
\begin{aligned}
s= & 1 \text { with } g_{i_{1}}=e^{\psi(\hbar, \kappa ; r, \theta)}, i_{1}=1,2 \\
& \psi^{\bullet \bullet}+\psi^{\prime \prime}=2{ }_{1}{ }_{1} \mathcal{K} ; \\
s= & 2 \text { with }\left\{\begin{array}{c}
\alpha_{i_{1}}=g_{4}^{\diamond} \partial_{i_{1}}\left({ }_{2} \varpi\right),{ }_{2} \beta=g_{4}^{\diamond}\left({ }_{2} \varpi\right)^{\diamond},{ }_{2} \gamma=\left(\ln \frac{\left|g_{4}\right|^{3 / 2}}{\left|g_{3}\right|}\right)^{\diamond} \\
\text { for }{ }_{2} \Psi=\exp \left({ }_{2} \varpi\right),{ }_{2} \varpi=\ln \mid g_{4}^{\diamond} / \sqrt{\left|g_{3} g_{4}\right|},
\end{array}\right. \\
& \left({ }_{2} \varpi\right)^{\diamond} g_{4}^{\diamond}=2 g_{3} g_{4}{ }_{2}^{\| 1} \mathcal{K}, \\
& { }_{2} \beta w_{j_{1}}-\alpha_{j_{1}}=0, \\
& n_{k_{1}}^{\diamond}+{ }_{2} \gamma n_{k_{1}}^{\diamond}=0 ;
\end{aligned}
$$




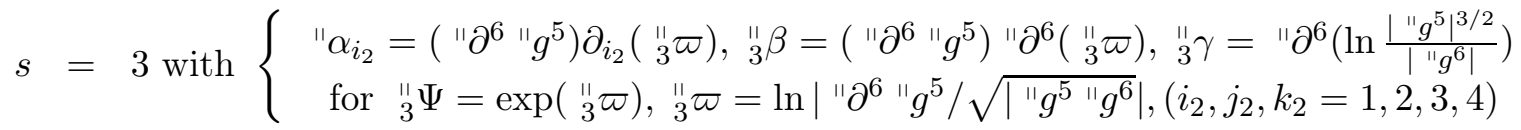

$$
\begin{aligned}
& \text { " } \partial^{6}\left({ }_{3}{ }_{3} \varpi\right) " \partial^{6} " g^{5}=2 \text { " } g^{5} " g^{6} \quad{ }_{3}{ }_{3} \mathcal{K}, \\
& \text { " } \partial^{66}\left(" n_{k_{2}}\right)+{ }_{3}^{1 "} \gamma "{ }^{\prime \prime} \partial^{6}\left(" n_{k_{2}}\right)=0 \text {, } \\
& { }_{3}^{\prime \prime} \beta \text { " } w_{j_{2}}-\text { " } \alpha_{j_{2}}=0 \text {; }
\end{aligned}
$$

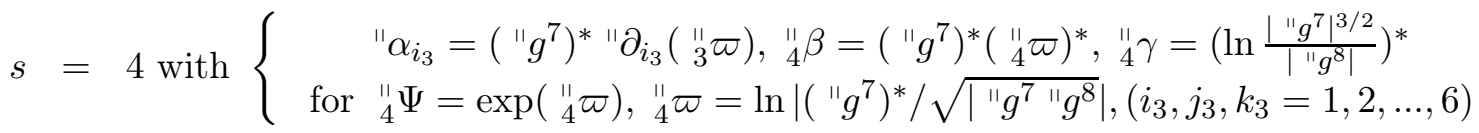

$$
\begin{aligned}
& \text { " } \partial^{6}\left({ }_{4}^{11} \varpi\right)\left({ }^{\prime \prime} g^{7}\right)^{*}=2 " g^{7} " g^{8}{ }_{4}{ }^{1} \widehat{\Upsilon}, \\
& \left({ }^{"} n_{k_{3}}\right)^{* *}+{ }_{4} \gamma\left(" n_{k_{3}}\right)^{*}=0, \\
& { }_{4}^{\prime \prime} \beta \text { " } w_{j_{3}}-"{ }^{\prime} \alpha_{j_{3}}=0 \text {. }
\end{aligned}
$$

Such a decoupling can be performed for real momentum variables $p_{a_{s}}$ using labels ${ }_{s}^{1}$ geometric s-objects but in nonassociative gravity/geometry certain coefficients contain the complex unity.

We can study independently a phase space dynamics and analyze it geometric evolution in various nonholonomic vacuum gravitational models for a quasi-stationary 4-d spacetime when parametric R-flux contributions (177) are encoded into the effective sources $" \mathbf{K}_{j_{2}}^{i_{2}}=\delta_{j_{2}}^{i_{2}}{ }_{s}^{\prime \prime} \mathcal{K}$, for $s=1,2$, and considering decoupling only of the system (86). In such cases, we have to solve recurrently the system of h1-v2 equations (88).

Above system of nonlinear PDEs possess an explicit decoupling property on every shell. For instance (when $s=1$ ), the first equation in (88) is a 2-d Poisson equation which can be solved in certain general forms for any prescribed source which, in this work, includes parametric R-flux contributions. We can integrate recurrently on every shell $s=2,3,4$ respective equations containing only partial derivatives on " $y^{3}$, " $p_{6}$, " $p_{8}$ for certain decoupled functions for the s-metric and N-connection coefficients. This can be performed in explicit form if there are prescribed any generating sources ${ }_{s} \mathcal{K}$ which for nonassociative gravity theories involve in parametric forms various R-flux and star deformation contributions.

\subsection{Generating stationary solutions for nonassociative vacuum phase spaces}

The nonassociative vacuum Einstein equations formulated in canonical dyadic variables on phase spaces can be integrated in some general forms following the results of [40], see Theorem 4.2 in section 4.3 .1 and proofs from appendix A.5. Similar examples and applications in 10-d string and Finsler-Lagrange-Hamilton geometry modifications of gravity (with details on AFCDM, locally anisotropic cosmological and BH solutions) are provided in Refs. [19, 20, 21]. In this work, we omit proofs because they can be obtained from respective constructions of cited work by re-defining dyadic variables on ${ }_{s} \mathbf{T}_{\|}^{*} \mathbf{V}$ and using parametric star deformed and R-flux induced sources ${ }_{s} \mathcal{K}$.

\subsubsection{Off-diagonal solutions with R-flux induced sources and noholonomic s-torsion}

By tedious straightforward computations, we can check that quasi-stationary solutions with Killing symmetry on $\partial /$ " $p_{7}$, for all shells $s=1,2,3,4$ and Killing symmetry on $\partial_{4}=\partial_{t}$ on spacetime shells $s=1,2$, for the nonassociative vacuum Einstein equations (70), with canonical Ricci s-tensors and effective sources decoupled in the form (88), are defined by such quadratic line elements on phase space:

$$
\begin{aligned}
d \widehat{s}^{2}= & e^{\psi\left(\hbar, \kappa ; x^{k_{1}}\right)}\left[\left(d x^{1}\right)^{2}+\left(d x^{2}\right)^{2}\right]+\frac{\left[\left({ }_{2} \Psi\right)^{\diamond}\right]^{2}}{4\left({ }_{2}{ }_{2} \mathcal{K}\right)^{2}\left\{g_{4}^{[0]}-\int d \varphi\left[\left({ }_{2} \Psi\right)^{2}\right]^{\diamond} / 4\left({ }_{2}{ }_{2} \mathcal{K}\right)\right\}}\left\{d \varphi+\frac{\partial_{i_{1}}\left({ }_{2} \Psi\right)}{\left({ }_{2} \Psi\right)^{\diamond}} d x^{i_{1}}\right\}^{2}+ \\
& \left\{g_{4}^{[0]}-\int d \varphi \frac{\left[\left({ }_{2} \Psi\right)^{2}\right]^{\diamond}}{4\left({ }_{2} \mathcal{K}\right)}\right\}\left\{d t+\left[{ }_{1} n_{k_{1}}+{ }_{2} n_{k_{1}} \int d \varphi \frac{\left[\left({ }_{2} \Psi\right)^{2}\right]^{\diamond}}{4\left({ }_{2}{ }_{2} \mathcal{K}\right)^{2}\left|g_{4}^{[0]}-\int d \varphi\left[\left({ }_{2} \Psi\right)^{2}\right]^{\diamond} / 4\left({ }_{2}{ }_{2} \mathcal{K}\right)\right|^{5 / 2}}\right] d x^{k_{1}}\right\}+
\end{aligned}
$$




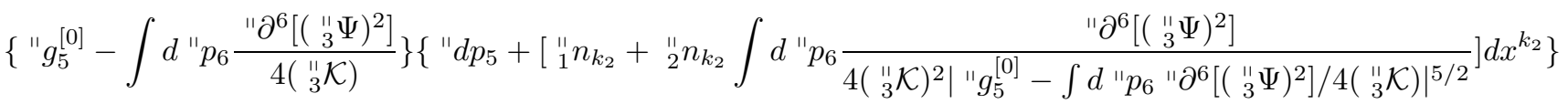

$$
\begin{aligned}
& +\frac{\left[" \partial^{6}\left({ }_{3}^{\prime \prime} \Psi\right)\right]^{2}}{4\left({ }_{3}^{\prime \prime} \mathcal{K}\right)^{2}\left\{" g_{5}^{[0]}-\int d " p_{6} " \partial^{6}\left[\left({ }_{3}^{\prime} \Psi\right)^{2}\right] / 4\left({ }_{3}^{\prime \prime} \mathcal{K}\right)\right\}}\left\{d " p_{6}+\frac{\partial_{i_{2}}\left({ }_{3}^{\prime \prime} \Psi\right)}{" \partial^{6}\left({ }_{3}^{\prime} \Psi\right)} d x^{i_{2}}\right\}^{2}+ \\
& \left\{" g_{7}^{[0]}-\int d " E \frac{\left[\left({ }_{4}^{\prime \prime} \Psi\right)^{2}\right]^{*}}{4\left({ }_{4}^{1 "} \mathcal{K}\right)}\right\}\left\{d " p_{7}+\left[{ }_{1}^{\prime \prime} n_{k_{3}}+{ }_{2}{ }_{2} n_{k_{3}} \int d " E \frac{\left[\left({ }_{4}^{\prime \prime} \Psi\right)^{2}\right]^{*}}{4\left({ }_{4}^{\prime \prime} \mathcal{K}\right)^{2}\left|" g_{7}^{[0]}-\int d " E\left[\left({ }_{4}^{\prime \prime} \Psi\right)^{2}\right]^{*} / 4\left({ }_{4}^{\prime \prime} \mathcal{K}\right)\right|^{5 / 2}}\right] d " x^{k_{3}}\right\}- \\
& \frac{\left[\left({ }_{4}^{\prime \prime} \Psi\right)^{*}\right]^{2}}{4\left({ }_{4}^{\prime \prime} \mathcal{K}\right)^{2}\left\{{ }^{\prime \prime} g_{7}^{[0]}-\int d " E\left[\left({ }_{4}^{\prime \prime} \Psi\right)^{2}\right]^{*} / 4\left({ }_{4}{ }_{4} \mathcal{K}\right)\right\}}\left\{d " E+\frac{" \partial_{i_{3}}\left({ }_{4}^{\prime \prime} \Psi\right)}{\left({ }_{4}^{\prime \prime} \Psi\right)^{*}} d " x^{i_{3}}\right\}^{2},
\end{aligned}
$$

for indices $i_{1}, j_{1}, k_{1}, \ldots=1,2 ; i_{2}, j_{2}, k_{2}, \ldots=1,2,3,4 ; i_{3}, j_{3}, k_{3}, \ldots=1,2, \ldots 6 ; y^{3}=\varphi, y^{4}=t$, " $p_{8}=$ " $E$; and

generating functions: $\psi\left(\hbar, \kappa ; x^{k_{1}}\right) ;{ }_{2} \Psi\left(\hbar, \kappa ; x^{k_{1}}, y^{3}\right) ; \quad{ }_{3}^{\prime \prime} \Psi\left(\hbar, \kappa ; x^{k_{2}}, \quad " p_{6}\right) ; \quad{ }_{4} \Psi\left(\hbar, \kappa ; " x^{k_{3}}, " E\right)$; generating sources: ${ }_{1}^{11} \mathcal{K}\left(\hbar, \kappa ; x^{k_{1}}\right) ; \quad{ }_{2}^{\|} \mathcal{K}\left(\hbar, \kappa ; x^{k_{1}}, y^{3}\right) ; \quad{ }_{3}^{\|} \mathcal{K}\left(\hbar, \kappa ; x^{k_{2}}, " p_{6}\right) ; \quad{ }_{4}^{" 1} \mathcal{K}\left(\hbar, \kappa ; " x^{k_{3}}, "{ }^{\prime \prime} E\right)$; integr. functions: $g_{4}^{[0]}\left(\hbar, \kappa ; x^{k_{1}}\right),{ }_{1} n_{k_{1}}\left(\hbar, \kappa ; x^{j_{1}}\right),{ }_{2} n_{k_{1}}\left(\hbar, \kappa ; x^{j_{1}}\right) ; " g_{5}^{[0]}\left(\hbar, \kappa ; x^{k_{2}}\right),{ }_{1} n_{k_{2}}\left(\hbar, \kappa ; x^{j_{2}}\right),{ }_{2} n_{k_{2}}\left(\hbar, \kappa ; x^{j_{2}}\right)$; $" g_{7}^{[0]}\left(\hbar, \kappa ; x^{j_{3}}\right),{ }_{1} n_{k_{3}}\left(\hbar, \kappa ; x^{j_{3}}\right),{ }_{2} n_{k_{3}}\left(\hbar, \kappa ; x^{j_{3}}\right)$.

Any generic off-diagonal solution ${ }_{s}^{\prime \prime} \widehat{\mathbf{g}}=" \widehat{\mathbf{g}}_{\alpha_{s} \beta_{s}}\left({ }_{s} x,{ }_{s}{ }_{s} p\right) " \widehat{\mathbf{e}}^{\alpha_{s}} \otimes{ }^{\prime \prime} \widehat{\mathbf{e}}^{\beta_{s}}(\overline{89})$ is exact but depends in parametric form on $\hbar, \kappa$ for any R-flux nonassociative data encoded in ${ }_{s}^{\prime} \mathcal{K}$.

Let us discuss the difference between certain classes of solutions of nonlinear systems of ODE (ordinary differential equations) and PDEs. Usually, physically important solutions in gravity theories (for instance, for $\mathrm{BH}$ solutions in GR) are found for certain diagonalize ansatz for metrics depending on one space (radial) variable. For such configurations, there are involved into considerations only four diagonal coefficients of a metric (from six independent ones; in 4-d spacetimes, a symmetric metric tensor contains 10 components but 4 of them can be transformed to zero values via coordinate transforms, which is consequence of Bianchi identities), see details in standard monographs on mathematical and general relativity and exact solutions [25, 24, 26, 27]. Under certain symmetry assumptions, the corresponding system of nonlinear PDEs (for instance, the vacuum Einstein equations) transforms into a second order nonlinear system of ODEs with radial derivatives. This can be integrated in exact form when the solutions are determined by two integration constants. If we consider a cosmological constant, we also introduce an (effective) source when the coefficients of diagonal static (or stationary, for rotating BHs) also depend on such a constant. The values of the integration constants are determined, for instance, from certain boundary/ asymptotic conditions, i.e. from some physical considerations, that the time like coefficient of the metric results in the Newton potential of a point mass.

When we consider systems of nonlinear PDEs of type (88) with ansatz (89), such nonassociative vacuum Einstein equations are not transformed into certain diagonal systems of nonlinear ODE. Such metrics depend, in general, on all spacetime and phase space coordinates even certain Killing symmetry conditions are imposed. As we have shown above, we can decouple such equations in very general forms when there are involved certain partial derivatives of the coefficients of metrics and if we work in some well defined nonholonomic dyadic bases and for corresponding canonical deformations of the nonlinear and linear connection structures. At the next step, we can integrate in explicit form the corresponding s-adapted systems of nonlinear PDEs. Performing such integrations we get dependencies not only on integration constants but also on integration functions depending on corresponding shell variables. More than that, such a geometric method of constructing exact solutions involves also generating functions and generating sources. Similar ideas are used in constructing solutions for 2-d and 3-d nonlinear solitonic equations but using the AFCDM there are involved different types of PDEs. Following such an approach, we construct certain general classes of solutions when generic off-diagonal (non) symmetric metrics and (generalized) connections depend, in principle, on all spacetime and phase coordinates but via respective integration functions and generating functions and sources. We have to consider additional physical motivations (generalized symmetries, causality principles, Cauchy type 
problems for certain evolution scenarios, or boundary/ asymptotic condition) for prescribing certain classes of generating functions and sources and how to determine the integration functions. One should be imposed certain additional conditions of compatibility with experimental/ observational data, to compute possible quantum corrections, various (non) associative/ commutative sources, star deformations etc. in order to elaborate on viable physical models.

In references [4, 17, 18, 5, 19, 20, 21, 40, we study typical geometric properties and various applications with constructing general integrals of systems of nonlinear PDEs in (modified) gravity if such configurations are constructed in some general forms and not only for special ansatz transforming PDEs into ODEs. It should be noted that the AFCDM is different from the well known Cartan's moving frame method and Newman-Penrose dyadic formalism considered in [25, 24, 26, 27]. Our approach is more general (for certain special conditions the AFCDM can be reduced to standard methods with ODEs in GR and MGTs) because involves additional distortions of the (non) linear connections structure. When certain classes of (off-) diagonal solutions have been constructed in a general form, we can impose additional nonholonomic constraints in order to extract, for instance, LC-configurations, subclasses of diagonal solutions, study parametric implications of some R-flux contributions, and to analyze how such configurations can be extended for complex variables, nonassociative and noncommutative symmetries, etc.

\subsubsection{Computing stationary coefficients for (non)symmetric star deformed s-metrics}

A complete parametric solution of nonassociative nonholonomic vacuum gravitational equations is determined also by nonsymmetric and symmetric components of a star deformed s-metric. Such values decouple and can be computed as induced by a solution ( $\widehat{\mathbf{g}}_{\alpha_{s} \beta_{s}}$, "“触) (89) if the effective sources " $\mathcal{s}$ K are subjected to nonholonomic constraints (80). Introducing such s-coefficients in formulas (71), we can compute the quasistationary canonical components of the nonsymmetric part of respective star deformed s-metric ${ }_{\star} \widehat{\mathfrak{g}}_{\alpha_{s}} \beta_{s}$,

$$
\begin{aligned}
& "{ }_{\star} \widehat{\mathfrak{a}}_{\alpha_{s} \beta_{s}}:=\quad\left[{ }_{\star}^{\prime \prime} \widehat{\mathfrak{a}}_{i_{1} j_{1}}=0,{ }_{\star}{ }^{\prime} \widehat{\mathfrak{a}}_{a_{2} b_{2}}=0,\right. \\
& { }_{\star} " \widehat{\mathfrak{a}}^{a_{3} b_{3}}=\frac{i \kappa}{2}\left(\overline{\mathcal{R}}_{c_{3}}^{n+k_{s} a_{3}} " \widehat{\mathbf{e}}_{k_{s}} " \widehat{\mathbf{g}}^{c_{3} b_{3}}-\overline{\mathcal{R}}_{c_{3}}^{n+k_{s} b_{3}} " \widehat{\mathbf{e}}_{k_{s}} " \widehat{\mathbf{g}}^{c_{3} a_{3}}\right)={ }_{\star}{ }^{\prime \prime} \widehat{\mathfrak{a}}_{[1]}^{a_{3} b_{3}}(\kappa)=\frac{1}{2}\left({ }_{\star}{ }_{\star} \widehat{\mathfrak{g}}_{[1]}^{a_{3} b_{3}}-{ }_{\star}{ }_{\star} \widehat{\mathfrak{g}}_{[1]}^{b_{3} a_{3}}\right)(\kappa),
\end{aligned}
$$

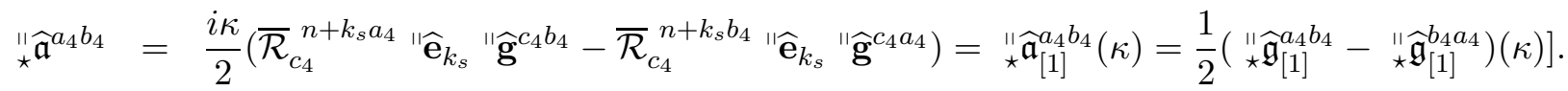

We omit cumbersome explicit formulas involving generating functions and sources, and integration functions from (89) which are encoded into the coefficients of s-metrics and s-frames with hat labels.

In a similar form, using any data ( $\widehat{\mathbf{g}}_{\alpha_{s} \beta_{s}}$, " $\widehat{\mathbf{e}}^{\alpha_{s}}$ ) (89) for (72), we can compute the symmetric part of a s-metric $\stackrel{\star}{\star} \widehat{\mathfrak{g}}_{\alpha_{s} \beta_{s}}$,

$$
\begin{aligned}
& " \widehat{\widetilde{\mathfrak{g}}}_{\alpha_{s} \beta_{s}}={ }^{\prime \widehat{\mathrm{g}}_{\alpha_{s} \beta_{s}}}+{ }_{\star}{ }_{\star} \widehat{\mathfrak{\mathfrak { g }}}_{\alpha_{s} \beta_{s}}^{[1]}(\kappa), \text { for }
\end{aligned}
$$

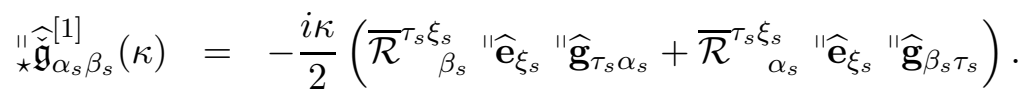

Another important value which can be computed for quasi-stationary solutions (89) is the real R-flux distortion on the spacetime which was considered as an effective source " $\widehat{\mathbf{K}} j_{2} k_{2}=-{ }_{[11]} \widehat{\mathbf{R}} i c_{j_{2} k_{2}}^{\star}\left(x^{k_{1}}, x^{3}\right)$ where all terms in formulas (77) are explicitly computed for some data ( $\widehat{\mathbf{g}}_{\alpha_{s} \beta_{s}}$, " $\widehat{\mathbf{e}}^{\alpha_{s}}$ ). Such way, we can define as determined by a solution (89) all s-adapted coefficients of canonical Ricci s-tensor $\widehat{\mathbf{R}} i c_{\alpha_{s}} \beta_{s}$ (24), respective star deformations to $\widehat{\mathbf{R}} i c_{\alpha_{s} \beta_{s}}^{\star}$ (45), and corresponding parametric decompositions (A.27)-(A.31), when all s-operators and coefficients are used for "hat" values in respective formulas. Certain values contain as multiples the complex unity. It is not clear what is the physical importance of such solutions which arise naturally in string gravity being induced by R-fluxes. In principle, we can consider generalizations of GR for complex spacetimes like in [42, 43] and then to involve complex models of nonholonomic metric-affine and Finsler-Lagrange-Hamilton like geometries [44] which were studied also in the framework of string theory 
[45, 46, 19]. The AFCDM can be extended for constructing exact and parametric solutions in such complex gravity models.

We can consider a subclass of h1-v2 solutions (89) generated by data

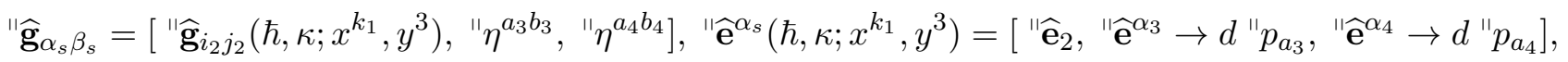

for $" \eta^{a_{3} b_{3}}=(1,1)$ and $" \eta^{a_{3} b_{3}}=(1,-1)$, when the integration and generation functions on shells $s=3,4$ are chosen for trivial N-connection structure, for instance with " $\widehat{N}_{j_{2}} a_{3}=0$, " $\widehat{N}_{j_{3} a_{4}}=0$ and prescribed c3-c4 sources ${ }_{3}^{\prime \prime} \mathcal{K}={ }_{4}^{\prime \prime} \mathcal{K}=0$. In result, we obtain quasi-stationary spacetime quadratic elements

$$
\begin{aligned}
d \widehat{s}^{2}= & e^{\psi\left(\hbar, \kappa ; x^{k_{1}}\right)}\left[\left(d x^{1}\right)^{2}+\left(d x^{2}\right)^{2}\right]+\frac{\left[\left({ }_{2} \Psi\left(\hbar, \kappa ; x^{k_{1}}, y^{3}\right)\right)^{\diamond}\right]^{2}}{4\left({ }_{2}{ }_{2} \mathcal{K}\left(\hbar, \kappa ; x^{k_{1}}, y^{3}\right)\right)^{2}\left\{g_{4}^{[0]}-\int d \varphi\left[\left({ }_{2} \Psi\left(\hbar, \kappa ; x^{k_{1}}, y^{3}\right)\right)^{2}\right]^{\diamond} / 4\left({ }_{2}{ }_{2} \mathcal{K}\left(\hbar, \kappa ; x^{k_{1}}, y^{3}\right)\right)\right\}} \\
& \left\{d \varphi+\frac{\partial_{i_{1}}\left({ }_{2} \Psi\left(\hbar, \kappa ; x^{k_{1}}, y^{3}\right)\right)}{\left({ }_{2} \Psi\left(\hbar, \kappa ; x^{k_{1}}, y^{3}\right)\right)^{\diamond}} d x^{i_{1}}\right\}^{2}+ \\
& \left\{g_{4}^{[0]}\left(\hbar, \kappa ; x^{k_{1}}\right)-\int d \varphi \frac{\left[\left({ }_{2} \Psi\left(\hbar, \kappa ; x^{k_{1}}, y^{3}\right)\right)^{2}\right]^{\diamond}}{4\left({ }_{2}{ }_{2} \mathcal{K}\left(\hbar, \kappa ; x^{k_{1}}, y^{3}\right)\right)}\right\}\left\{d t+\left[{ }_{1} n_{k_{1}}\left(\hbar, \kappa ; x^{k_{1}}\right)+\right.\right. \\
& \left.\left.{ }_{2} n_{k_{1}}\left(\hbar, \kappa ; x^{k_{1}}\right) \int d \varphi \frac{\left[\left({ }_{2} \Psi\left(\hbar, \kappa ; x^{k_{1}}, y^{3}\right)\right)^{2}\right]^{\diamond}}{4\left({ }_{2}^{11} \mathcal{K}\left(\hbar, \kappa ; x^{k_{1}}, y^{3}\right)\right)^{2}\left|g_{4}^{[0]}-\int d \varphi\left[\left({ }_{2} \Psi\left(\hbar, \kappa ; x^{k_{1}}, y^{3}\right)\right)^{2}\right]^{\diamond} / 4\left({ }_{2}{ }_{2} \mathcal{K}\left(\hbar, \kappa ; x^{k_{1}}, y^{3}\right)\right)\right|^{5 / 2}}\right] d x^{k_{1}}\right\}
\end{aligned}
$$

defining exact solutions of the systems of nonassociative canonical nonholonomic vacuum Einstein equations (81) reduced to (86) with trivial (87). The quadratic elements (92) encode via effective generating sources ${ }_{1}^{1} \mathcal{K}$ and ${ }_{2} \mathcal{K}$ nontrivial R-flux contributions. Similar generic off-diagonal metrics were studied in GR and various (non) commutative modifications [17, 18, 19] but for different types of effective sources. Star deformations of such solutions are symmetric up to 1st order on $\kappa$ as follow from formulas (90) and (91).

So, prescribing any generating quasi-stationary sources " $\widehat{\mathbf{K}}_{\beta_{s}}^{\alpha_{s}}$ (69) with nonassociative parametric deformations and using block $[(2 \times 2)+(2 \times 2)]+[(2 \times 2)+(2 \times 2)]$ parametrization of symmetric and nonsymmetric

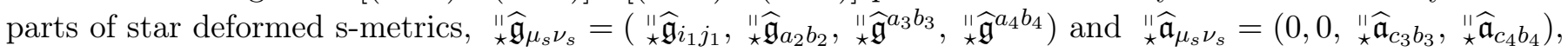
we can compute R-flux deformations for any stated associative (off-) diagonal quasi-stationary solution " $\widehat{\mathrm{g}}_{\alpha_{s}} \beta_{s}$. The corresponding quadratic elements are considered on phase space to be of type (89) and reduces on spacetime in the form (92).

\subsubsection{Extracting nonassociative parametric LC-configurations}

Quasi-stationary solutions constructed in previous subsection are with nontrivial canonical s-torsion structure ${ }_{s}^{\prime} \widehat{\mathbf{T}}_{\alpha_{s} \beta_{s}}^{\gamma_{s}}$ of respective ${ }_{s}^{\prime \prime} \widehat{\mathbf{D}}=" \nabla+{ }_{s} \mid \widehat{\mathbf{Z}}$. We have to impose additional nonholonomic constraints in the type of generating functions and effective sources in order to satisfy the conditions ${ }_{s} \widehat{\mathbf{Z}}$ and extract zero torsion LC-configurations for " $\nabla$ defined on phase space and/ or for respective constraints on spacetimes with nontrivial positive R-flux structure. The solutions (89) and (92) can be constrained in such a form that up to orders $\hbar, \kappa$ and $\hbar \kappa$, for star deformations of respective connections, there are satisfied the conditions ${ }_{\star}^{\| s} \widehat{\mathbf{Z}}=0$, i. e. ${ }_{s}^{\prime \prime} \widehat{\mathbf{D}}_{|s|}^{\star} \widehat{\mathbf{T}=0}=" \nabla^{\star}$, see formulas (42) and (41)) (we have to consider additional nonholonomic shell by shell constraints for higher order parametric terms).

By straightforward computations on cotangent bundles (see section 4.3.2 in [40], for similar details and reference on previous works), we can verify that all canonical s-torsion coefficients vanish if the coefficients of a quasi-stationary s-metric, and respective coefficients of N-connections, see formulas for ansatz (82), are subjected to satisfy such equations,

$$
\begin{array}{ll}
s=2: & w_{i_{1}}^{\diamond}=\mathbf{e}_{i_{1}} \ln \sqrt{\left|g_{3}\right|}, \mathbf{e}_{i_{1}} \ln \sqrt{\left|g_{4}\right|}=0, \partial_{i_{1}} w_{j_{1}}=\partial_{j_{1}} w_{i_{1}} \text { and } n_{i_{1}}^{\diamond}=0 ; \\
s=3: & \quad " \partial^{6} " n_{i_{2}}=\mathbf{e}_{i_{2}} \ln \sqrt{\left|" g^{6}\right|}, \quad \mathbf{e}_{i_{2}} \ln \sqrt{\left|" g^{5}\right|}=0, \partial_{i_{2}} n_{j_{2}}=\partial_{j_{2}} n_{i_{2}} \text { and } \quad " \partial^{6} w_{i_{2}}=0 ; \\
s=4: & \quad n_{i_{3}}^{*}=" \mathbf{e}_{i_{3}} \ln \sqrt{\left|" g^{8}\right|}, \quad " \quad \mathbf{e}_{i_{3}} \ln \sqrt{\left|" g^{7}\right|}=0, \quad " \partial_{i_{3}} " n_{j_{3}}=" \partial_{j_{3}} \quad " n_{i_{3}} \text { and " } n_{i_{3}}^{*}=0 ;
\end{array}
$$




$$
\begin{aligned}
& \text { and } s=2: \quad n_{k_{1}}\left(x^{i_{1}}\right)=0 \text { and } \partial_{i_{1}} n_{j_{1}}\left(x^{k_{1}}\right)=\partial_{j_{1}} n_{i_{1}}\left(x^{k_{1}}\right) \text {; } \\
& s=3: \quad w_{k_{2}}\left(x^{i_{2}}\right)=0 \text { and } \partial_{i_{2}} w_{j_{2}}\left(x^{k_{2}}\right)=\partial_{j_{2}} w_{i_{2}}\left(x^{k_{2}}\right) ; \\
& s=4: \quad \text { " } w_{k_{3}}\left("{ }^{i_{3}}\right)=0 \text { and } \quad " \partial_{i_{3}} " w_{j_{3}}\left(" x^{k_{3}}\right)="{ }^{\prime} \partial_{j_{3}} " w_{i_{3}}\left(" x^{k_{3}}\right) \text {. }
\end{aligned}
$$

The solutions for $w$ - and $n$-functions which can be obtained for (93) depend in parametric form on $\hbar, \kappa$ on the class of vacuum or non-vacuum s-metrics encoding possible nonassociative effective sources. So, they relate and constrain additionally such s-metric coefficients to possible R-flux contributions. We state some important conditions on respective classes of generating functions and generating sources which result in zero s-torsions on every shell:

First, it should be noted that if we prescribe a shell generating function ${ }_{2} \Psi={ }_{2} \check{\Psi}\left(\hbar, \kappa, x^{i_{1}}, y^{3}\right)$, for which $\left[\partial_{i_{1}}\left({ }_{2} \check{\Psi}\right)\right]^{\diamond}=\partial_{i_{1}}\left({ }_{2} \check{\Psi}\right)^{\diamond}$, we solve the conditions for $w_{j_{1}}$ in (93) in explicit form when the effective source ${ }_{2}^{11} \mathcal{K}=$ const, or if such an effective source can be expressed as a functional $\left.{ }_{2}^{\prime \prime} \mathcal{K}\left(\hbar, \kappa, x^{i}, y^{3}\right)={ }_{2} \mathcal{K}_{2} \check{\Psi}\right]$. Similar conditions should be formulated for $s=3,4$. For instance, we prescribe ${ }_{3}^{11} \Psi={ }_{3}^{11} \Psi\left(\hbar, \kappa, x^{i_{2}}, p^{6}\right)$, for which $" \partial^{6}\left[\partial_{i_{2}}(\quad \stackrel{4}{3})\right]=\partial_{i_{2}} " \partial^{6}\left(\begin{array}{c}\| \\ 3\end{array}\right)$.

We note that the third conditions in (93), for $s=2$, i.e. $\partial_{i_{1}} w_{j_{1}}=\partial_{j_{1}} w_{i_{1}}$, can be solved in parametric form for any generating function ${ }_{2} \check{A}={ }_{2} \check{A}\left(\hbar, \kappa, x^{k}, y^{3}\right)$ for which

$$
w_{i_{1}}=\check{w}_{i_{1}}=\partial_{i_{1} 2} \Psi /\left({ }_{2} \Psi\right)^{\diamond}=\partial_{i_{1}}{ }_{2} \check{A} .
$$

On the second shell, we consider similar formulas but with duality in order to involve configurations with nontrivial dependence on energy type variable,

$$
n_{i_{2}}=\check{n}_{i_{2}}=\partial_{i_{2}}{ }_{3}^{\prime \prime} \Psi / " \partial^{6}\left({ }_{3}^{\prime \prime} \Psi\right)=\partial_{i_{2}}{ }_{3}^{\prime \prime} \check{A} .
$$

Summarizing above formulas, we obtain such LC-conditions for generating functions and generating sources:

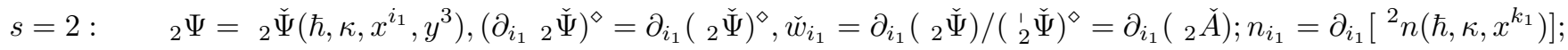

$$
\begin{aligned}
& { }_{2}^{11} \mathcal{K}\left(\hbar, \kappa, x^{i}, y^{3}\right)={ }_{2}^{11} \mathcal{K}\left[\begin{array}{c}
1 \\
2
\end{array} \bar{\Psi}\right] \text {, or } \quad{ }_{2}^{\prime \prime} \mathcal{K}=\text { const; } \\
& s=3: \quad{ }_{3}^{11} \Psi={ }_{3}^{1} \check{\Psi}\left(\hbar, \kappa, x^{i_{2}}, " p^{6}\right), " \partial^{6}\left[\partial_{i_{2}}\left({ }_{3}^{\prime \prime} \check{\Psi}\right)\right]=\partial_{i_{2}} " \partial^{6}\left({ }_{3}^{\prime \prime} \check{\Psi}\right) ; \check{w}_{i_{2}}=\partial_{i_{2}}\left({ }_{3}^{\prime \prime} \Psi\right) / " \partial^{6}\left({ }_{3}^{\prime \prime} \Psi\right)=\partial_{i_{2}}\left({ }_{3}^{\prime \prime} \check{A}\right) ; \\
& n_{i_{2}}=\partial_{i_{2}}\left[{ }^{3} n\left(\hbar, \kappa, x^{k_{2}}\right)\right] ;{ }_{3}^{11} \mathcal{K}\left(\hbar, \kappa, x^{i_{2}}, " p^{6}\right)={ }_{3}^{11} \mathcal{K}\left[\begin{array}{l}
1 \\
3
\end{array} \check{\Psi}\right] \text {, or } \quad{ }_{3}^{11} \mathcal{K}=\text { const; }
\end{aligned}
$$

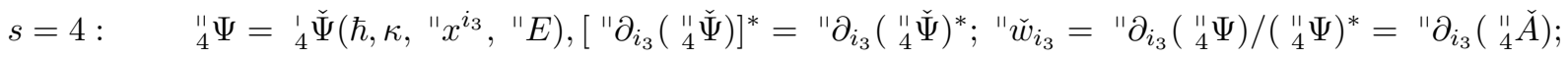

$$
\begin{aligned}
& " n_{i_{3}}=" \partial_{i_{3}}\left[{ }^{4} n\left(\hbar, \kappa, "{ }^{k_{3}}\right)\right] ;{ }_{4} \mathcal{K}\left(\hbar, \kappa, x^{i_{3}}, " E\right)={ }_{4}^{"} \mathcal{K}\left[{ }_{4}^{\prime \prime} \check{\Psi}\right] \text {, or } \quad{ }_{4} \mathcal{K}=\text { const. }
\end{aligned}
$$

For such subclasses of s-coefficients, the quadratic line element (89) transforms into

$$
\begin{aligned}
d \widehat{s}_{L C s t}^{2}= & \text { " } \widehat{\hat{g}}_{\alpha \beta}\left(\hbar, \kappa, " x^{k_{3}},{ }^{"} E\right) d^{" \prime} u^{\alpha} d " u^{\beta}=e^{\psi\left(\hbar, \kappa, x^{k_{1}}\right)}\left[\left(d x^{1}\right)^{2}+\left(d x^{2}\right)^{2}\right]+ \\
& \frac{\left[\left({ }_{2} \check{\Psi}\right)^{\diamond}\right]^{2}}{4\left({ }_{2} \mathcal{K}\left[{ }_{2} \check{\Psi}\right]\right)^{2}\left\{g_{4}^{[0]}-\int d \varphi\left[\left({ }_{2} \check{\Psi}\right)^{2}\right]^{\diamond} / 4\left({ }_{2}{ }_{2} \mathcal{K}\right)\right\}}\left\{d \varphi+\left[\partial_{i_{1}}\left({ }_{2} \check{A}\right)\right] d x^{i_{1}}\right\}+ \\
& \left\{g_{4}^{[0]}-\int d \varphi \frac{\left[\left({ }_{2} \check{\Psi}\right)^{2}\right]^{\diamond}}{4\left({ }_{2}{ }_{2} \mathcal{K}\left[{ }_{2} \check{\Psi}\right]\right)}\right\}\left\{d t+\partial_{i_{1}}\left[{ }^{2} n\left(x^{k_{1}}\right)\right] d x^{i_{1}}\right\}+
\end{aligned}
$$




$$
\begin{aligned}
& \left\{" g_{5}^{[0]}-\int d " p_{6} \frac{\partial^{6}\left[\left({ }_{3}^{\prime \prime} \Psi\right)^{2}\right]}{4\left({ }_{3}^{11} \mathcal{K}\left[{ }_{3}^{1} \check{\Psi}\right]\right)}\right\}\left\{d " p_{5}+\partial_{i_{2}}\left[{ }^{3} n\left(x^{k_{2}}\right)\right] d x^{i_{2}}\right\}+
\end{aligned}
$$

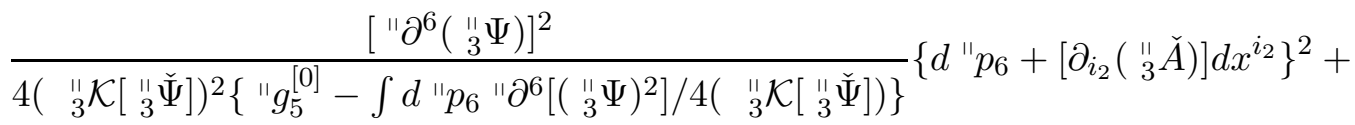

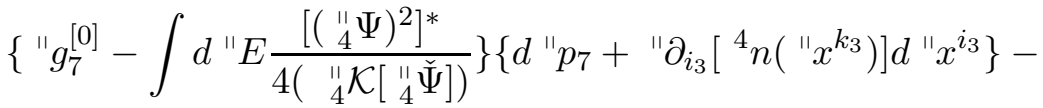

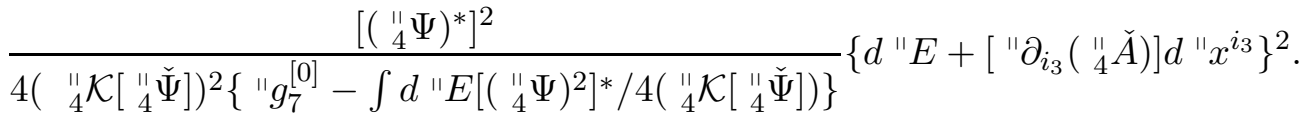

The first four terms for shells $s=1,2$ in (95) define a LC-variant of (92) for quasi-stationary spacetime solutions with R-flux contributions encoded in ${ }_{1}^{\|} \mathcal{K}$ and ${ }_{2}^{1} \mathcal{K}$. This way, we generate exact solutions of the systems of nonassociative vacuum Einstein equations (81) for " $\nabla^{\star}$ and reduced to (86) with trivial (87) and " $\nabla^{\star} \rightarrow \nabla^{\star}$. Such nonassociative vacuum equations with nontrivial real R-flux contributions were postulated in [2. Redefining and generalizing those systems of nonlinear PDEs in nonholonomic dyadic frames, we can apply the AFCDM and generate exact and parametric solutions as we explained above.

Finally, we emphasize that quasi-stationary s-metrics (95) encoding R-flux effective sources are generic off-diagonal if at least on one of the four shells there are nontrivial anholonomy relations of type (4). On phase space, such exact solutions possess a Killing symmetry on " $\partial^{7}$, i.e. there are such $\mathrm{N}$-adapted coordinate systems when the s-metrics do not depend on coordinate " $p_{7}$ but may depend generically on an energy type (complex) variable " $E$. We generate quasi-stationary configurations if the generating and integration functions and generating sources encoding star and R-flux deformations on respective shells are chosen to not depend on the $t$-coordinate. In dual form, redefining the AFCDM in a form to generate solutions with Killing symmetry, for instance, on $\partial_{3}$, we can construct various classes of locally cosmological solutions, see details and references in [20, 4, 5]. For models of nonassociative gravity, we shall construct and study nonassociative cosmological models in our partner works.

\subsection{Nonassociative nonlinear symmetries for stationary generating functions and R-flux sources}

Applying the AFCDM for generating exact and parametric solutions in (non) associative / commutative gravity theories with (non) symmetric metrics and generalized connections, we discover a new type of nonlinear symmetries of nonholonomic gravitational and matter field systems with one Killing symmetry [14, 15, 16, 20, 4, 5. In this subsection, we analyze how such nonlinear symmetries relate generating functions and (effective) sources in the case of quasi-stationary solutions (89), (92), and restrictions to LC-configurations (95). Considering nonlinear symmetries, we can redefine the generating functions and introduce on dyadic shells some nontrivial effective cosmological constants ${ }_{s}^{\prime \prime} \Lambda_{0}=$ const instead of effective sources ${ }_{s} \mathcal{K}\left({ }_{s}^{\prime \prime} u\right)$. We can work also with effective polarizations of cosmological constants for some functions ${ }^{s} \Lambda\left({ }_{s} u\right)$, as we explain with respect to formulas (63), (64), and (65). The redefined generating functions encode, for instance, R-flux contributions and parametric star deformations. Fixing certain values ${ }_{s} \Lambda_{0}$, allows us to express off-diagonal solutions in a more simplified form and compute possible parametric nonassociative deformations of physically important solutions (for instance, BHs, wormholes and homogeneous and isotropic cosmological configurations nonholonomically modified into locally anisotropic ones, which is used in our partner works).

On the shell $s=2$, we can change the generating data,

$$
\left({ }_{2} \Psi\left(\hbar, \kappa, x^{i_{1}}, y^{3}\right), \quad{ }_{2} \mathbb{K}\left(\hbar, \kappa, x^{i_{1}}, y^{3}\right)\right) \leftrightarrow\left({ }_{2} \Phi\left(\hbar, \kappa, x^{i_{1}}, y^{3}\right),{ }_{2} \Lambda_{0}\right),
$$


following formulas

$$
\begin{aligned}
\frac{\left[\left({ }_{2} \Psi\right)^{2}\right]^{\diamond}}{{ }_{2}{ }_{2} \mathcal{K}} & =\frac{\left[\left({ }_{2} \Phi\right)^{2}\right]^{\diamond}}{{ }_{2} \Lambda_{0}}, \text { which can be integrated as } \\
\left.{ }_{2} \Phi\right)^{2} & ={ }_{2} \Lambda_{0} \int d x^{3}\left({ }_{2}{ }_{2} \mathcal{K}\right)^{-1}\left[\left({ }_{2} \Psi\right)^{2}\right]^{\diamond} \text { and } / \text { or }\left({ }_{2} \Psi\right)^{2}=\left({ }_{2} \Lambda_{0}\right)^{-1} \int d x^{3}\left({ }_{2}{ }_{2} \mathcal{K}\right)\left[\left({ }_{2} \Phi\right)^{2}\right]^{\diamond} .
\end{aligned}
$$

In result, we can express (see similar computations in detail in section 4.4 of [40]),

$$
\begin{aligned}
& g_{3}\left[{ }_{2} \Psi\right]=-\frac{\left[\left({ }_{2} \Psi\right)^{\diamond}\right]^{2}}{4\left({ }_{2} \mathcal{K} \mathcal{K}\right)^{2} g_{4}\left[{ }_{2} \Psi\right]}=g_{3}\left[{ }_{2} \Phi\right]=-\frac{1}{g_{4}\left[{ }_{2} \Phi\right]} \frac{\left({ }_{2} \Phi\right)^{2}\left[\left({ }_{2} \Phi\right)^{\diamond}\right]^{2}}{\mid{ }_{2} \Lambda_{0} \int d y^{3}\left({ }_{2} \mathcal{K} \mathcal{K}\right)\left[\left({ }_{2} \Phi\right)^{2}\right]^{\diamond \mid}} \text {, where } \\
& g_{4}\left[{ }_{2} \Psi\right]=g_{4}^{[0]}-\int d \varphi \frac{\left[\left({ }_{2} \Psi\right)^{2}\right]^{\diamond}}{4\left({ }_{2}^{\prime \prime} \mathcal{K}\right)}=g_{4}\left[{ }_{2} \Phi\right]=g_{4}^{[0]}-\frac{\left({ }_{2} \Phi\right)^{2}}{4{ }_{2} \Lambda} \text {. } \\
& w_{i_{1}}\left(x^{k_{1}}, y^{3}\right)=\frac{\partial_{i_{1}}\left({ }_{2} \Psi\right)}{\left({ }_{2}^{1} \Psi\right)^{\diamond}}=\frac{\partial_{i_{1}}\left[\left({ }_{2} \Psi\right)^{2}\right]}{\left[\left({ }_{2}^{11} \mathcal{K}\right)^{2}\right]^{\diamond}}=\frac{\partial_{i_{1}} \int d y^{3}\left({ }_{2}{ }_{2} \mathcal{K}\right)\left[\left({ }_{2} \Phi\right)^{2}\right]^{\diamond}}{\left({ }_{2} \mathcal{K}\right)\left[\left({ }_{2} \Phi\right)^{2}\right]^{\diamond}} ; \text { and } \\
& n_{k_{1}}\left(x^{k_{1}}, y^{3}\right)={ }_{1} n_{k_{1}}+{ }_{2} n_{k_{1}} \int d y^{3} \frac{g_{3}\left[{ }_{2} \Phi\right]}{\left|g_{4}\left[{ }_{2} \Phi\right]\right|^{3 / 2}} \\
& ={ }_{1} n_{k_{1}}+{ }_{2} n_{k_{1}} \int d y^{3}\left(\frac{\left({ }_{2} \Psi\right)^{\diamond}}{2{ }_{2}^{\prime \prime} \mathcal{K}}\right)^{2}\left|g_{4}^{[0]}\left(x^{k_{1}}\right)-\int d y^{3} \frac{\left[\left({ }_{2} \Psi\right)^{2}\right]^{\diamond}}{4\left({ }_{2}{ }_{2} \mathcal{K}\right)}\right|^{-5 / 2} \\
& ={ }_{1} n_{k_{1}}+{ }_{2} n_{k_{1}} \int d y^{3} \frac{\left({ }_{2} \Phi\right)^{2}\left[\left({ }_{2} \Phi\right)^{\diamond}\right]^{2}}{\mid{ }_{2} \Lambda_{0} \int d y^{3}\left({ }_{2} \mathcal{K}\right)\left[\left({ }_{2} \Phi\right)^{2}\right]^{\diamond \mid}}\left|g_{4}^{[0]}-\frac{\left({ }_{2} \Phi\right)^{2}}{4{ }_{2} \Lambda_{0}}\right|^{-5 / 2} \text {. }
\end{aligned}
$$

Similar re-definitions of generating functions and sources (96) and (97) can be considered for $s=3,4$ which allows us to prove that any quasi-stationary solution (89) of the nonassociative nonholonomic dyadic vacuum gravitational equations on phase spaces for the canonical s-connection is characterized by such important nonlinear symmetries:

$$
\begin{aligned}
& s=2: \quad \frac{\left[\left({ }_{2} \Psi\right)^{2}\right]^{\diamond}}{{ }_{2}^{11} \mathcal{K}}=\frac{\left[\left({ }_{2} \Phi\right)^{2}\right]^{\diamond}}{{ }_{2} \Lambda_{0}} \\
& \text { i.e. }\left({ }_{2} \Phi\right)^{2}={ }_{2} \Lambda_{0} \int d x^{3}\left({ }_{2}^{11} \mathcal{K}\right)^{-1}\left[\left({ }_{2} \Psi\right)^{2}\right]^{\diamond} \text { and } / \text { or }\left({ }_{2} \Psi\right)^{2}=\left({ }_{2} \Lambda_{0}\right)^{-1} \int d x^{3}\left({ }_{2}{ }_{2} \mathcal{K}\right)\left[\left({ }_{2} \Phi\right)^{2}\right]^{\diamond} \text {; } \\
& s=3: \quad \frac{\| \partial^{6}\left[\left({ }_{3}^{\prime \prime} \Psi\right)^{2}\right]}{{ }_{3}^{11} \mathcal{K}}=\frac{" \partial^{6}\left[\left({ }_{3}{ }_{3} \Phi\right)^{2}\right]}{{ }_{3} \Lambda_{0}}, \\
& \text { i.e. } \quad\left({ }_{3}^{\prime \prime} \Phi\right)^{2}={ }_{3}^{\prime \prime} \Lambda_{0} \int d " p_{6}\left({ }_{3}^{\prime \prime} \mathcal{K}\right)^{-1}\left[\left({ }_{3}^{\prime \prime} \Psi\right)^{2}\right] \text { and } / \text { or }\left({ }_{3}^{\prime \prime} \Psi\right)^{2}=\left({ }_{3}^{\prime \prime} \Lambda_{0}\right)^{-1} \int d " p_{6}\left({ }_{3}^{\prime \prime} \mathcal{K}\right)\left[\left({ }_{3}^{\prime \prime} \Phi\right)^{2}\right] \text {; } \\
& s=4: \quad \frac{\left[\left({ }_{4}^{\prime \prime} \Psi\right)^{2}\right]^{*}}{{ }_{4}^{" 1} \mathcal{K}}=\frac{\left[\left({ }_{4}^{" 1} \Phi\right)^{2}\right]^{*}}{{ }_{4}^{" 1} \Lambda}
\end{aligned}
$$

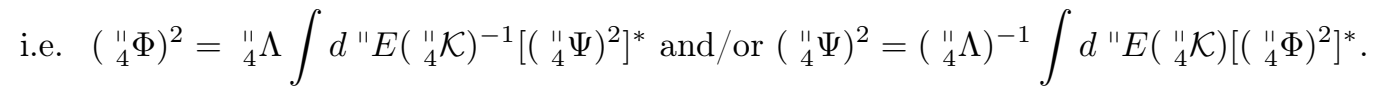

Using nonlinear symmetries (98), we can write the quadratic element (89) for off-diagonal quasi-stationary nonholonomic vacuum solutions with Killing symmetry on " $p_{7}$ can be written in equivalent form encoding 
the nonlinear symmetries of generating functions, generating sources of R-flux origin and respective effective shell cosmological constants,

$$
\begin{aligned}
& d \widehat{s}^{2}=" g_{\alpha_{s} \beta_{s}}\left(\hbar, \kappa, x^{k}, y^{3}, " p_{a_{3}}, " E, \quad{ }_{s} \Phi,{ }_{s}{ }_{s} \Lambda_{0}\right) d " u^{\alpha_{s}} d^{"} u^{\beta_{s}}=e^{\psi\left(\hbar, \kappa, x^{k_{1}}\right)}\left[\left(d x^{1}\right)^{2}+\left(d x^{2}\right)^{2}\right] \\
& -\frac{\left({ }_{2} \Phi\right)^{2}\left[\left({ }_{2} \Phi\right)^{\diamond}\right]^{2}}{\left|{ }_{2} \Lambda_{0} \int d x^{3}\left({ }_{2}^{11} \mathcal{K}\right)\left[\left({ }_{2} \Phi\right)^{2}\right]^{\diamond}\right|\left[g_{4}^{[0]}-\left({ }_{2} \Phi\right)^{2} / 4{ }_{2} \Lambda_{0}\right]}\left\{d x^{3}+\frac{\partial_{i_{1}} \int d x^{3}\left({ }_{2}{ }_{2} \mathcal{K}\right)\left[\left({ }_{2} \Phi\right)^{2}\right]^{\diamond}}{\left({ }_{2}^{11} \mathcal{K}\right)\left[\left({ }_{2} \Phi\right)^{2}\right]^{\diamond}} d x^{i_{1}}\right\}^{2}- \\
& \left\{g_{4}^{[0]}-\frac{\left({ }_{2} \Phi\right)^{2}}{4{ }_{2} \Lambda_{0}}\right\}\left\{d t+\left[{ }_{1} n_{k_{1}}+{ }_{2} n_{k_{1}} \int d y^{3} \frac{\left({ }_{2} \Phi\right)^{2}\left[\left({ }_{2} \Phi\right)^{\diamond}\right]^{2}}{\left|{ }_{2} \Lambda_{0} \int d y^{3}\left({ }_{2}{ }_{2} \mathcal{K}\right)\left[\left({ }_{2} \Phi\right)^{2}\right]^{\diamond}\right|\left[g_{4}^{[0]}-\left({ }_{2} \Phi\right)^{2} / 4{ }_{2} \Lambda_{0}\right]^{5 / 2}}\right]\right\}+
\end{aligned}
$$

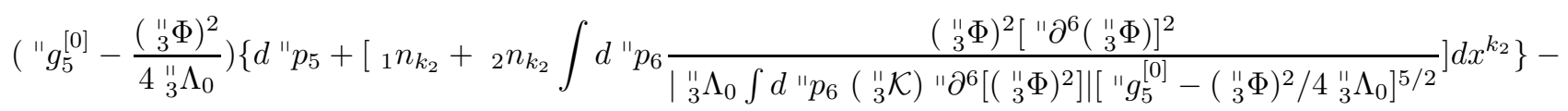

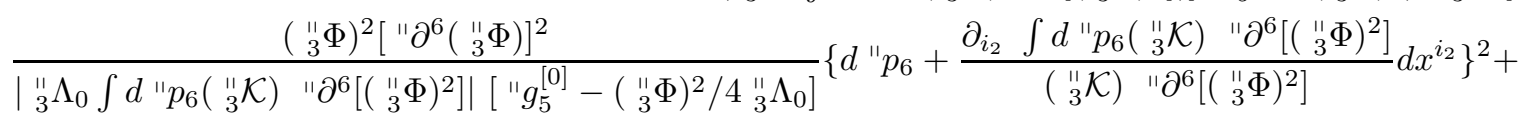

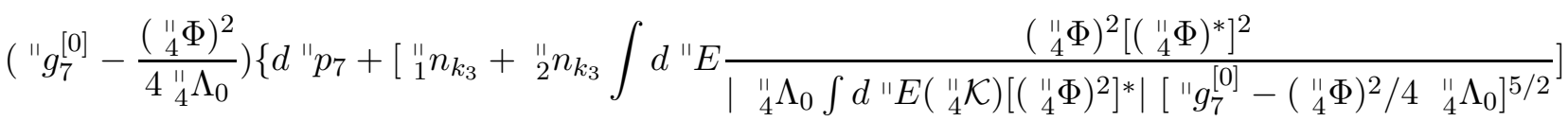

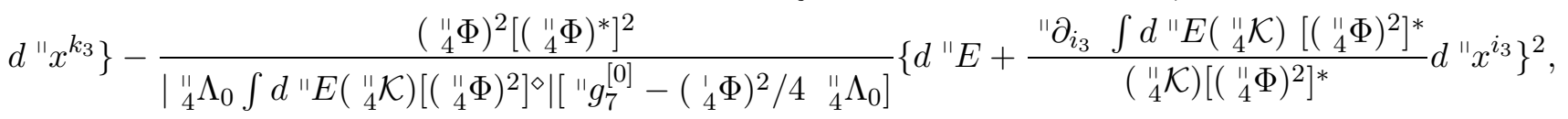

for indices: $i_{1}, j_{1}, k_{1}, \ldots=1,2 ; i_{2}, j_{2}, k_{2}, \ldots=1,2,3,4 ; i_{3}, j_{3}, k_{3}, \ldots=1,2, \ldots 6 ; x^{3}=\varphi, y^{4}=t$, " $p_{8}=$ " $E$; and

generating functions: $\psi\left(\hbar, \kappa, x^{k_{1}}\right) ;{ }_{2} \Phi\left(\hbar, \kappa, x^{k_{1}} y^{3}\right) ;{ }_{3}{ }_{3} \Phi\left(\hbar, \kappa, x^{k_{2}}, "{ }_{6}\right) ;{ }_{4}{ }_{4} \Phi\left(\hbar, \kappa, " x^{k_{3}}, " \mathrm{E}\right)$;

generating sources: ${ }_{1}^{11} \mathcal{K}\left(\hbar, \kappa, x^{k_{1}}\right) ; \quad{ }_{2}^{11} \mathcal{K}\left(\hbar, \kappa, x^{k_{1}}, y^{3}\right) ; \quad{ }_{3}^{11} \mathcal{K}\left(\hbar, \kappa, x^{k_{2}}, \quad " p_{6}\right) ; \quad{ }_{4} \mathcal{K}\left(\hbar, \kappa, " x^{k_{3}}, " E\right)$;

integr. functions: $g_{4}^{[0]}\left(\hbar, \kappa, x^{k_{1}}\right),{ }_{1} n_{k_{1}}\left(\hbar, \kappa, x^{j_{1}}\right),{ }_{2} n_{k_{1}}\left(\hbar, \kappa, x^{j_{1}}\right) ; " g_{5}^{[0]}\left(\hbar, \kappa, x^{k_{2}}\right),{ }_{1} n_{k_{2}}\left(\hbar, \kappa, x^{j_{2}}\right),{ }_{2} n_{k_{2}}\left(\hbar, \kappa, x^{j_{2}}\right)$;

" $g_{7}^{[0]}\left(\hbar, \kappa, "{ }^{j_{3}}\right),{ }_{1}^{"} n_{k_{3}}\left(\hbar, \kappa, " x^{j_{3}}\right),{ }_{2} n_{k_{3}}\left(\hbar, \kappa, " x^{j_{3}}\right)$.

In a similar form, we can re-define the generating functions and introduce effective cosmological constants ${ }_{s} \Lambda_{0}$ for LC-configurations and quasi-stationary s-metrics (95).

For convenience, we present the Appendix B where the procedure of integrating nonassociative nonholonomic vacuum gravitational equations is studied in details for the case of effective source encoding R-flux and star deformation parametric terms. We provide a nonassociative generalization of section 5 in [40] following such goals:

1. We show how certain coefficients of quasi-stationary s-metrics can be chosen as shell generating functions which is important for constructing new classes of exact and parametric solutions with R-flux deformations from certain nonholonomic but associative/ commutative physically important solutions.

2. The AFCDM is re-formulated for conventional gravitational polarization functions for nonholonomic deformations of arbitrary s-metrics (they can be nonassociative or associative/commutative ones) into certain new classes of exact solutions determining general quasi-stationary nonassociative vacuum configurations.

3. A small parametric decomposition formalism is elaborated for off-diagonal deformations and diagonalization procedures for generating new classes of exact and parametric solutions encoding nonassociative R-flux contributions on phase spaces and spacetime configurations.

4. There are studied three examples of generic off-diagonal metrics for canonical and Levi-Civita "pure" vacuum configurations (62) when possible real cosmological constants are compensated by effective ones, for instance, by R-flux contributions. 


\section{Conclusions}

In this work, we have developed the anholonomic frame and connection deformation method, AFCDM [40, 21, 20, 22, 23, in a nonholonomic dyadic form which allows us to prove a general decoupling and integration property of nonassociative vacuum gravitational equations introduced in [1, 2]. The star product deformation approach to geometric and "non-geometric" models with R-flux contributions from string theories was completed with a nonassociative nonholonomic geometric formalism involving nonsymmetric and symmetric metric structures and generalized (non) linear connections in the first our partner paper [3]. A very important result proven in [2] for parametric decompositions on $\hbar$ and $\kappa$ (respectively, the Planck and string constants) is that there are nontrivial real R-flux contributions on background (associative and commutative) pseudo-Riemannian spacetimes. In our approach, such nonassociative parametric deformations are encoded in effective sources and, via nonlinear symmetries, related to corresponding classes of generating function and prescribed values of shell constants.

Nonassociative extensions of GR with parametric R-flux deformations are defined on phase spaces which can be modelled as cotangent Lorents bundles enabled with additional algebraic (for instance, quasi-Hopf / octonion / quaternion structures), star products, complexified momentum like coordinates, etc. On real, and commutative background, phase spaces, such extensions of general relativity and string gravity theories are characterized by modified dispersion relations, MDRs, of type $c^{2} \overrightarrow{\mathbf{p}}^{2}-E^{2}+c^{4} m^{2}=\varpi(E, \overrightarrow{\mathbf{p}}, m ; \hbar, \kappa)$, see details and motivations for nonassociative gravity in [3]. Such modified gravity theories, MGTs, with MDRs determined by indicators $\varpi\left(x^{i}, E, \overrightarrow{\mathbf{p}}, m ; \ell_{P}\right)$, where $m$ is a mass parameter and $\ell_{P}$ is the Planck lengths, were axiomatized and studied in [4, 5], see also references therein. On commutative and noncommutative generalized Einstein, Finsler-Lagrange-Hamilton and Einsten-Eisenhart-Moffat models, we cite respectively [6, 17, 8, 9, 10, 11, 12, 13, 14, 15, 16, etc.). The metric structure on such (non) associative/ commutative phase spaces depends not only on spacetime coordinates but also on some conventional velocity / momentum like variables, for instance, with effective metrics $g_{\alpha \beta}\left(x^{k}, p_{a}\right)$, which for R-flux contributions deform into symmetric and nonsymmetric metric structures adapted to certain classes of nonlinear connections determined by respective MDR indicators.

To construct exact and parametric solutions in nonassociative gravity and geometric flow theories, which for various classes of above mentioned (non) associative/ commutative MGTs are characterized by generic diagonal metrics, auxiliary canonical d- and s-connections and respective nonholonomic constraints to zero torsion LC-configurations, we can apply the results of Theorems 4.1- 4.3 and 5.1, 5.2 from [40. Considering phase spaces with momentum variables multiplied to complex unity and effective R-flux sources, we summarize the main results of this article:

1. Nonassociative vacuum Einstein equations can be decoupled in general off-diagonal forms with symmetric and nonsymmetric star metrics determined by star parametric R-flux deformations, when the solutions depend on all phase and spacetime coordinates being determined by respective classes of generating functions and (effective) sources and integration functions.

2. Quasi-stationary off-diagonal solutions with one phase space Killing symmetry and time-like spacetime Killing symmetry on the first two nonholonomic dyadic shell can be constructed in splicit forms. Using additional nonholonomic constraints, we can extract LC-configurations, define spacetime projections encoding R-flux contributions, perform diagonalization procedures etc.

3. We defined new types of nonassociative nonlinear parametric symmetries relating generating functions, generating effective R-flux sources, and certain effective shell cosmological constants which are important for constructing exact and parametric solutions with generic off-diagonal symmetric and nonsymmetric background and star deformed metrics, generalized connections and zero torsion configurations.

4. Small parametric decompositions on $\hbar$ and $\kappa$ in nonassociative vacuum gravity have a natural R-flux 
deformation interpretation and can be performed in a self-consistent form defining star nonholonomic deformations of certain prime (associative) metrics into new classes of target symmetric and nonsymetric metrics.

We note that the AFCDM can be applied for constructing real (with all types local signature) and complex solutions for vacuum and non-vacuum generalized (non) associative/ commutative nonholonomic gravitational and matter field equations as we reviewed in [4, 5, 3. In general, it is not clear how to evaluate the physical importance of complex R-flux contributions into parametric decompositions of Ricci tensors, effective sources, and (non) symmetric metrics. Nevertheless, we can always impose such nonholonomic constraints when complex terms vanish for certain $\mathrm{N}$ - / s-adapted configurations and the star R-flux deformations result in real terms and decoupling of respective physically important nonlinear systems of PDEs. An alternative way, is to work in so called Finsler-Lagrange-Hamilton variables with almost complex/ symplectic structures which allow a real relativistic interpretation of nonassociative nonholonomic gravity and geometric flow theories. Here we also note that such constructions can be used for deformation, nonholonomic gauge like and A-brane quantization of nonassociative gravity theories as we considered in our previous works, see reviews of results in [17, 44, 18, 41, 4].

Finally, we note that the geometric formalism and analytic methods on decoupling and integrating in general form nonassociative gravity theories elaborated in this and first partner work [3] will be applied in a series of related partner papers (under elaboration) on nonassociative black hole solutions, locally anisotroic cosmological solutions with nonassociatvie quasi-periods and phase space/ spacetime quasi-crystal structure and theory of nonassociative geometric and quantum information flows. For associative models and geometric methods, we cite our previous works [19, 20, 22, 23].

Acknowledgments: This work develops for nonassociative geometry and gravity some research programs on geometry and physics, during 2006-2015, supported by senior fellowships at the Perimeter Institute and Fields Institute (Ontario, Canada), CERN (Geneva, Switzerland) and Max Planck Institut für Physik / Werner Heisenberg Institut, München (Germany). SV is grateful to professors V. G. Kupriyanov, D. Lüst, N. Mavromatos, J. Moffat, D. Singleton and P. Stavrinos for respective hosting of short/ long terms visits, seminars, and/or discussing important ideas and preliminary results.

\section{A Nonassociative differential geometry with s-connections \& nonsymmet- ric metrics}

In this appendix, we re-formulate the nonassociative differential geometry with N-connections and nonsymmetric metrics [3] in dyadic s-variables involving quasi-Hopf structure. For holonomic configurations with zero cosmological constants and effective sources, we obtain geometric models from [2]. We omit constructions with s-transforms from some subsections of section 3 in [3], which define nonholonomic generalizations of nonassociative geometry elaborated in [1]. Such formulas can be obtained if we re-define respective frame, tensor product and star product transforms (i.e. of " $\mathbf{e}_{\alpha_{s}} \rightarrow " \partial_{\alpha_{s}}, \otimes_{\star s} \rightarrow \otimes_{\star}, \star_{s} \rightarrow \star$ etc.). There are provided abstract (with s-labels) and s-adapted coefficient formulas, with respect to nonholonomic dyadic frames, for star deformed connections, s-connections, and sketched the proofs of formulas for their torsion and curvature dyadic shell distinguished tensors, s-tensors.

\section{A.1 Dyadic s-adapted linear connections and quasi-Hopf s-structures}

The (non) associative d-connection formalism elaborated in section 3 of partner work [3] can be extended for s-tensors and differential forms on the $\mathcal{A}_{s}^{\star}$-bimodule $V e c_{\star_{s}}$ of s-vector fields and the $\Omega_{s \star}^{\natural}-$ bimodule $V e c_{\star_{s}}^{\natural}=$ $V e c_{\star_{s}} \otimes_{\star_{s}} \Omega_{s \star}^{\natural}\left(\mathcal{M},{ }_{s}^{"} N\right)$ as we considered in subsection 2.3 .2 , 
For a dyadic with $s=1,2,3,4$, a star s-connection can be defined as a s-adapted linear map $h$

$$
\begin{aligned}
& { }_{s} \mathbf{D}^{\star}: V e c_{\star_{s}} \rightarrow V e c_{\star_{s}} \otimes_{\star_{s}} \Omega_{\star}^{1} \text {, i. e. } \mathbf{v} \rightarrow{ }_{s}^{" 1} D^{\star} " \mathbf{v}={ }^{\prime \prime} \mathbf{v}^{\alpha_{s}} \otimes_{\star_{s}} " \omega_{\alpha_{s}}=\left(v^{i_{s}} \otimes_{\star_{s}} \omega_{i_{s}}, " v_{a_{s}} \otimes_{\star_{s}} " \omega^{a_{s}}\right) \text {; } \\
& { }_{s}^{\|} \mathbf{D}^{\star}=\left({ }_{h_{1}}^{\|} \mathbf{D}^{\star},{ }_{v_{2}}^{\|} \mathbf{D}^{\star},{ }_{c_{3}}^{\|} \mathbf{D}^{\star},{ }_{c_{4}}^{\|} \mathbf{D}^{\star}\right): V e c_{\star_{s}}=\left(h_{1} V e c_{\star_{s}}, v_{2} V e c_{\star_{s}}, c_{3} V e c_{\star_{s}}, c_{4} V e c_{\star_{s}}\right) \rightarrow \\
& \left(h_{1} V e c_{\star_{s}}, v_{2} V e c_{\star_{s}}, c_{3} V e c_{\star_{s}}, c_{4} V e c_{\star_{s}}\right) \otimes_{\star_{s}}\left(h_{1} \Omega_{\star}^{\natural}, v_{2} \Omega_{\star}^{\natural}, c_{3} \Omega_{\star}^{\natural}, c_{4} \Omega_{\star}^{\natural}\right),
\end{aligned}
$$

with h- / v-, c-splitting and for "v ${ }^{\alpha_{s}} \otimes_{\star_{s}} " \omega_{\alpha_{s}} \in V e c_{\star_{s}} \otimes_{\star_{s}} \Omega_{s \star}^{1}$.

Here we note that the adjoint action of a s-vector ${ }_{s} \xi_{s} \xi \in V e c^{\mathcal{F}}\left(\mathcal{M},{ }_{s} N\right)$ results in a s-adapted linear map ${ }_{s \xi} \mathbf{D}^{\star}: V e c_{\star_{s}} \rightarrow V e c_{\star_{s}} \otimes_{\star_{s}} \Omega_{\star}^{1}$, which is also a s-connection. This property exists for all types of s-connections in nonassociative nonholonomic geometry when an infinite number of covariant star nonholonomic calculi can be elaborated. We shall follow a "minimal" way for a s-connection ${ }_{s} \mathbf{D}^{\star}$ (A.1) with corresponding extensions to a s-adapted covariant derivative for s-vector filed values in the exterior s-algebra $V e c_{\star s}^{\natural}=V e c_{\star s} \otimes_{\star s} \Omega_{s \star}^{\natural}$, see details for N-configurations in section 3.1.2 of [3]. We omit such a tedious geometric dyadic calculus which in local coordinate (co) bases is similar to formula (4.8) in [2]. Usually, it is possible to follow a geometric symbolic rule when the constructions with quasi-Hopf algebras and respective nonassociative covariant calculus can be extended in $\mathrm{N}$ - and s-adapted forms when (using notations from that and our works), for instance, $\partial \rightarrow " \mathbf{e} \rightarrow{ }_{s}^{\prime \prime} \mathbf{e}, \nabla_{z}^{\star} \rightarrow{ }^{\prime \prime} \mathbf{D} \rightarrow{ }_{s}^{\prime \prime} \mathbf{D}$ and with actions of absolute differential operators $d_{\nabla_{z}^{\star}} \rightarrow{ }_{\mathbf{D}^{\star}} \mathbf{d} \rightarrow{ }_{\mathbf{s D}^{\star}} \mathbf{d}$.

For a star deformed d-connection (A.1), the s-adapted coefficients ${ }_{s}^{\prime \prime} \mathbf{D}^{\star}=\left\{" \boldsymbol{\Gamma}_{\star \beta_{s}}^{\alpha_{s}}, \in \mathcal{A}_{s}^{\star}\right\}$ are computed following such definitions and formulas

$$
\begin{aligned}
& { }_{s}^{"} \mathbf{D}^{\star} " \mathbf{e}_{\alpha_{s}}:=\quad \text { "} \mathbf{e}_{\beta_{s}} \otimes_{\star_{s}} " \boldsymbol{\Gamma}_{\star \alpha_{s}}^{\beta_{s}}, \text { where } \\
& " \boldsymbol{\Gamma}_{\star \beta_{s}}^{\alpha_{s}}=" \boldsymbol{\Gamma}_{\star \beta_{s} \gamma_{s}}^{\alpha_{s} \star_{s}} \text { "e } \mathbf{e}^{\gamma_{s}} \text { is a star s-connection 1-form; in brief, }{ }_{\mathbf{s} \star} \boldsymbol{\Gamma}={ }_{\star} \boldsymbol{\Gamma}_{\gamma_{s}} \star_{s} "{ } \mathbf{e}^{\gamma_{s}} \text {. }
\end{aligned}
$$

Using " $\mathbf{D}^{\star}{ }_{\mathbf{e}_{\beta s}}:=" \mathbf{D}^{\star}{ }_{\beta_{s}}$, we obtain

$$
" \mathbf{D}_{\alpha_{s}}^{\star}{ } " \mathbf{e}_{\beta_{s}}:=\left\langle{ }_{s}^{\prime \prime} \mathbf{D}^{\star} " \mathbf{e}_{\beta_{s}}, \quad " \mathbf{e}_{\alpha_{s}}\right\rangle_{\star_{s}}=\left\langle\left(" \mathbf{e}_{\gamma_{s}} \otimes_{\star_{s}}\left(" \boldsymbol{\Gamma}_{\star \beta_{s} \tau_{s}}^{\gamma_{s}} \star_{s} " \mathbf{e}^{\tau_{s}}\right)\right), \quad " \mathbf{e}_{\alpha_{s}}\right\rangle_{\star_{s}}=" \mathbf{e}_{\gamma_{s} \star_{s}} " \boldsymbol{\Gamma}_{\star \beta_{s} \alpha_{s}}^{\gamma_{s}} .
$$

The s-connection coefficients $" \boldsymbol{\Gamma}_{\star \beta_{s} \gamma_{s}}^{\alpha_{s}}$ and respective dyadic 1-form ${ }_{s} \boldsymbol{\Gamma}_{\star}$ are used for computing s-adapted covariant derivatives:

$$
\begin{aligned}
& { }_{s}^{"} \mathbf{D}^{\star}{ }_{s} \mathbf{v}=" \mathbf{e}_{\gamma_{s}} \otimes_{\star_{s}}\left({ }_{s}^{\|} \mathbf{d} " \mathbf{v}^{\gamma_{s}}+{ }^{n} \boldsymbol{\Gamma}_{\star \alpha_{s}}^{\gamma_{s} \star_{s}} "{ }^{\alpha_{s}}\right) \text {, for s-vector }{ }_{s}^{\prime \prime} \mathbf{v}=" \mathbf{e}_{\alpha_{s} \star_{s}} " \mathbf{v}^{\alpha_{s}}, "{ }^{\alpha_{s}} \in \mathcal{A}_{s}^{\star} ; \\
& { }_{s \mathbf{D}^{\star}} \mathbf{d}\left({ }^{\prime \prime} \mathbf{e}_{\gamma_{s}} \otimes_{\star_{s}} " \omega^{\gamma_{s}}\right)={ }^{\prime} \mathbf{e}_{\gamma_{s}} \otimes_{\star_{s}}\left(" \mathbf{d} " \omega^{\gamma_{s}}+{ }^{"} \boldsymbol{\Gamma}_{\star \alpha_{s}}^{\gamma_{s} \star_{s}} "{ }^{\prime \alpha_{s}}\right) \text {, for " } \omega^{\alpha_{s}} \in \Omega_{s \star}^{\natural} \text {. }
\end{aligned}
$$

We define s-components of a star deformed s-connection by introducing " $\mathbf{e}_{i_{s}}$ and "e $e^{b_{s}}$ in formulas (A.2) - (A.3). For instance, we obtain a dyadic splitting for " $\mathbf{D}^{\star} k_{s} " e^{b_{s}}=" e^{a_{s} \star_{s}} " L_{\star a_{s} k_{s}}^{b_{s}}$. This way, acting on respective adapted (co) bases, we obtain a s-decomposition,

$$
\begin{aligned}
& { }_{s}{ }_{s} \mathbf{D}^{\star}=\left\{{ }^{\|} \boldsymbol{\Gamma}_{\star \alpha_{s} \beta_{s}}^{\gamma_{s}}=\left({ }^{\prime \prime} L_{\star j_{1} k_{1}}^{i_{1}}, \quad " L_{\star b_{2} k_{1}}^{a_{2}}, \quad " C_{\star j_{1} c_{2}}^{i_{1}}, \quad " C_{\star b_{2} c_{2}}^{a_{2}} ;\right.\right.
\end{aligned}
$$

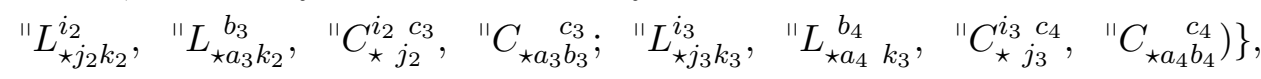

where $i_{1}, j_{1}, k_{1}=1,2$ (such indices are h-indices) $; a_{2}, b_{2}, c_{2}=3,4$ (such indices are $v$-indices) $; i_{2}, j_{2}, ; k_{2}=$ $1,2,3,4$ (such indices for 1,2 are h-indices and, for 3,4 , are v-indices); $a_{2}, b_{2}, c_{2}=5,6$ (such indices a cindices); $i_{3}, j_{3}, k_{3}=1,2,3,4,5,6$ (such indices for 1,2 are h-indices; for 3,4, are v-indices; for 5,6, are cindices) $; a_{4}, b_{4}, c_{4}=7,8$ (such indices are also c-indices).

Similar s-adapted coefficients can be computed for star s-deformations of $T_{s} \mathbf{T V}$ and $T_{s} \mathbf{T}^{*} \mathbf{V}$ but we omit such formulas which are similar to (A.4) with respective changing of v- to c-indices, and labels "'" " $\rightarrow$ "'", for respective shall, or not putting such duality symbols if not necessary.

For quasi-Hopf s-structures, we can also consider left actions of covariant s-derivatives, which can be formulated in terms of the dual star s-connection ${ }_{s \star}^{\prime} \mathbf{D}$. Such details were considered in a subsection 
related to formula (66) in [3] for the case of dual star d-connections. We omit dubbing of those constructions for s-structures because all formulas can be obtained by geometric similarity. In abstract geometric form but using only holonomic bases, such constructions are elaborated in sections 4.2 and 4.3 of [2]. Respective s-adapted coefficient formulas can be written by geometric analogy and/or derived for explicit actions on s-elongated (co) bases as we computed above.

\section{A.2 Star generalizations of the torsion, Riemann and Ricci s-tensors}

We show how such geometric constructions can be performed in abstract s-adapted coefficient forms using $\mathrm{N}$-adapted coefficient formulas from section 3 in [3] generalizing in noholonomic (if necessary, dyadic) forms respective definitions and holonomic formulas from [2].

\section{A.2.1 Nonassociative star s-torsions for s-connections and quasi-Hopf s-structures}

A star deformed s-torsion ${ }_{s \star}^{11} \mathcal{T} \in V e c_{\star_{N}} \otimes_{\star_{N}} \Omega_{\star}^{2}$ of nonassociative s-connection ${ }_{s}^{\prime \prime} \mathbf{D}^{\star}$ (A.1) can be defined as a s-adapted map with identity $\left\langle " \mathbf{e}_{\alpha_{s}} \otimes_{\star_{s}} " \mathbf{e}^{\alpha_{s}},\right\rangle_{\star_{s}}: V e c_{\star_{s}} \rightarrow V e c_{\star_{s}}$, which can be expanded to any svector field "v $=" \mathbf{e}_{\alpha_{s}} \star_{s} " \mathbf{v}^{\alpha_{s}}, " \mathbf{v}^{\alpha_{s}} \in \mathcal{A}_{N}^{\star}$, when the associator acts trivially on any basis (co) s-vector $" \mathbf{e}_{\alpha_{s}}=\left(" \mathbf{e}_{i_{s}}, " e^{a_{s}}\right)$ (5). Using the s-adapted absolute differential ${ }_{s \mathbf{D}^{\star}} \mathbf{d}$ (A.3), we define and compute

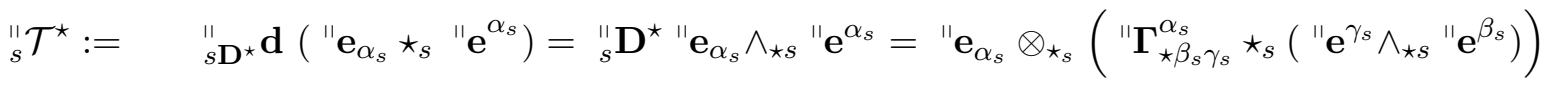

$$
\begin{aligned}
& :=\quad " \mathbf{e}_{\alpha_{s}} \otimes_{\star_{s}}{ }_{\star}^{\|} \mathcal{T}^{\alpha_{s}} \text {, where } \\
& { }_{\star} \mathcal{T}^{\alpha_{s}}:=" \boldsymbol{\Gamma}_{\star \beta_{s} \gamma_{s} \star_{s}}^{\alpha_{s}}\left(" \mathbf{e}^{\gamma_{s}} \wedge_{\star s} " \mathbf{e}^{\beta_{s}}\right)=\left(" \Gamma_{\star \beta_{s} \gamma_{s}}^{\alpha_{s}}-" \boldsymbol{\Gamma}_{\star \gamma_{s} \beta_{s}}^{\alpha_{s}}+"{ }^{\alpha_{\star} \gamma_{s} \beta_{s}}\right) \star_{s}\left(" \mathbf{e}^{\gamma_{s}} \star_{s} " \mathbf{e}^{\beta_{s}}\right) \text {, for } \\
& {\left[" \mathrm{e}_{\gamma_{s}}, " \mathbf{e}_{\beta_{s}}\right]_{\star_{s}}=" \mathbf{e}_{\gamma_{s}} \wedge_{\star s} " \mathbf{e}_{\beta_{s}}=" \mathbf{e}_{\gamma_{s} \star_{s}} " \mathbf{e}_{\beta_{s}}-" \mathbf{e}_{\beta_{s} \star_{s}} " \mathbf{e}_{\gamma_{s}}="{ }^{\alpha_{\star}} \mathrm{w}_{s} \beta_{s} \star_{s} " \mathbf{e}_{\alpha_{s}} \text {. }}
\end{aligned}
$$

A star s-torsion is also a s-adapted map ${ }_{s}^{\|} \mathcal{T}^{\star}: V e c_{\star_{s}} \otimes_{\star_{s}} V e c_{\star_{s}} \rightarrow V e c_{\star_{s}}$, when

$$
{ }_{s} \mathcal{T}^{\star}(" \mathbf{z}, " \mathrm{v})=\left\langle{ }_{s} \mathcal{T}^{\star}, \quad " \mathbf{z} \otimes_{\star_{s}} " \quad \mathbf{v}\right\rangle_{\star_{s}}=" \mathbf{e}_{\alpha_{s}} \star_{s}\left\langle{ }_{\star} \mathcal{T}^{\alpha_{s}}, \quad " \mathbf{z} \otimes_{\star_{s}} \quad " \mathbf{v}\right\rangle_{\star_{s}},
$$

for some s-vectors "z and "v. The conditions of right $\mathcal{A}_{s}^{\star}$-linearity and star antisymmetry, ${ }_{s \star} \mathcal{T}($ " $\mathbf{z}, " \mathbf{v})=$ - ${ }_{s \star} \mathcal{T}\left({ }_{T} \mathbf{v},{ }_{T} \mathbf{z}\right)$, are also satisfied, where the braiding d-operator " ${ }_{T}$ " is used for actions of type

$$
\tau_{\mathcal{R}}\left(" \mathbf{z} \otimes_{\star_{\mathrm{s}}} " \mathrm{v}\right)=\left(\begin{array}{l}
\| \\
\mathrm{T}
\end{array}\right.
$$

This results in formulas,

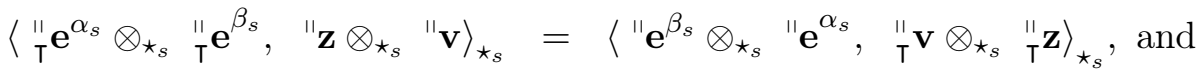

$$
\begin{aligned}
& \left\langle " \mathbf{e}^{\alpha_{s}} \wedge_{\star_{s}} " \mathbf{e}^{\beta_{s}}, \quad " \mathbf{z} \otimes_{\star_{s}} " \mathbf{v}\right\rangle_{\star_{s}}=\left\langle " \mathbf{e}^{\alpha_{s}} \otimes_{\star_{s}} " \mathbf{e}^{\beta_{s}}, \quad " \mathbf{z} \wedge_{\star_{s}} " \mathbf{v}\right\rangle_{\star_{s}} .
\end{aligned}
$$

Using the s-operator ${ }_{s}^{\|} \mathcal{T}^{\star}($ A.5. $)$ on $" \mathbf{e}_{\alpha_{s}}=\left(" \mathbf{e}_{i_{s}}, " e^{a_{s}}\right)$ (15), we compute the d-torsion coefficients for $" \mathbf{z}=" \mathbf{e}_{\alpha_{s}}$ and $" \mathbf{v}=" \mathbf{e}_{\beta_{s}}$,

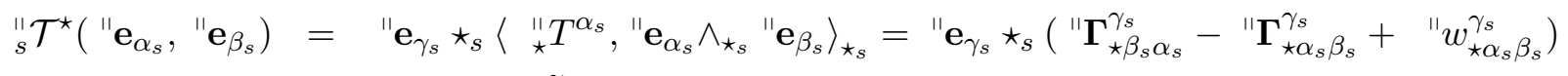

$$
\begin{aligned}
& =\quad " \mathbf{e}_{\gamma_{s} \star_{s}}{ }^{\prime \prime} \mathbf{T}_{\star \beta_{s} \alpha_{s}}^{\gamma_{s}} \text {, }
\end{aligned}
$$

see formulas (A.5). The s-coefficient N-adapted decomposition of s-torsion is parameterized in the form

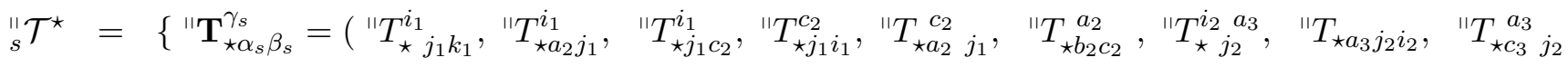

$$
\begin{aligned}
& \text { " } \left.\left.T_{\star a_{3}}^{b_{3} c_{3}}, " \quad T_{\star}^{i_{3} a_{3}},{ }^{i_{4}}, T_{\star a_{4} j_{3} i_{3}}, \quad " T_{\star c_{4} j_{3}}^{a_{4}}, \quad " T_{\star a_{4}}^{b_{4} c_{4}}\right)\right\},
\end{aligned}
$$


where the s-adapted coefficients are computed

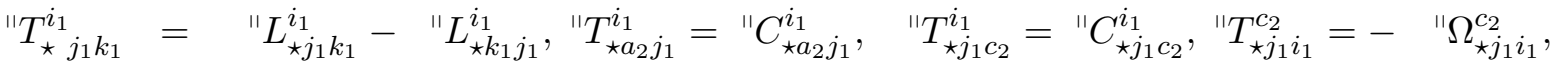

$$
\begin{aligned}
& " T_{\star a_{2} j_{1}}^{c_{2}}=\quad L_{\star a_{2} j_{1}}^{c_{2}}-" e_{a_{2}}\left(" N_{\star j_{1}}^{c_{2}}\right), \quad " T_{\star b_{2} c_{2}}^{a_{2}}=" C_{\star b_{2} c_{2}}^{a_{2}}-{ }^{\prime} C_{\star c_{2} b_{2}}^{a_{2}} ;
\end{aligned}
$$

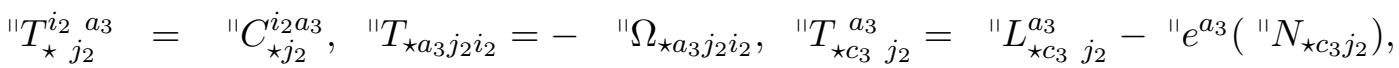

$$
\begin{aligned}
& " T_{\star a_{3}}^{b_{3} c_{3}}=" C_{\star a_{3}}^{b_{3} c_{3}}-{ }^{\prime \prime} C_{\star a_{3}}^{c_{3} b_{3}},
\end{aligned}
$$

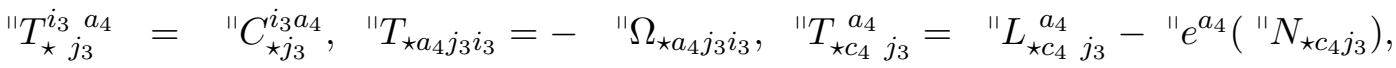

$$
\begin{aligned}
& " T_{\star a_{4}}^{b_{4} c_{4}}={ }^{\prime} C_{\star a_{4}}^{b_{4} c_{4}}-{ }^{"} C_{\star a_{4}}^{c_{4} b_{4}},
\end{aligned}
$$

when ${ }_{s} \mathbf{D}^{\star}$ is determined by s-components as in formulas (A.4).

For " $\mathbf{z}=" \mathbf{e}_{\alpha_{s}}, " \mathbf{v}={ }^{\prime} \mathbf{e}_{\beta_{s}}$ and $" \mathbf{D}_{\mathrm{T} \alpha_{s}}^{\star}=" \mathbf{D}_{\mathrm{T}}^{\star} \mathbf{e}_{\alpha_{s}}$, we compute

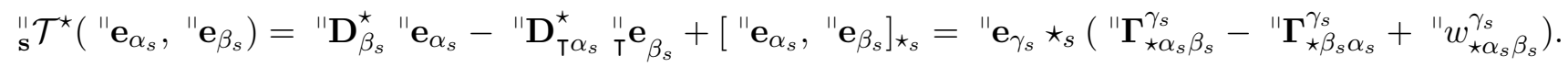

This defines a $U \operatorname{Vec}^{\mathcal{F}}\left(\mathcal{M},{ }_{s} N\right)$-equivariant s-operator and can be written in terms of s-adapted associative composition $\bullet$, pairing and braiding $\tau_{\mathcal{R}}$ (A.6),

$$
{ }_{s}^{\|} \mathcal{T}^{\star}(,)=\langle,\rangle_{\star s} \bullet\left({ }_{\mathbf{s}} \mathbf{D}^{\star} \otimes_{\star_{s}} i d\right)-\langle,\rangle_{\star_{s}} \bullet\left({ }_{s} \mathbf{D}^{\star} \otimes_{\star_{s}} i d\right) \bullet \tau_{\mathcal{R}}+[,]_{\star_{s}},
$$

when ${ }_{s}^{\prime \prime} \mathcal{T}^{\star}(" \mathbf{z}, " \mathbf{v})={ }_{s}^{\|} \mathcal{T}^{\star}\left(" \mathbf{z} \otimes_{\star_{\mathbf{s}}} " \mathbf{v}\right)$.

\section{A.2.2 Star deformed dyadic curvature of quasi-Hopf s-adapted structures}

We extend for s-connections and s-adapted frames the definitions and formulas the star deformed curvature s-tensors. For holonomic basic structures such constructions are provided in section 4.5 of [2] and for Ndecompositions in section 3.2 of [3].

\section{Abstract definition of the curvature s-tensor and s-adapted differential forms:}

We can define the curvature of a $\mathrm{N}$-adapted covariant derivative ${ }_{s \mathbf{D}^{\star}} \mathbf{d}(\underline{\mathrm{A} .3})$ as the s-operator

$$
{ }_{s} \Re^{\star}:={ }_{s \mathbf{D}^{\star}}^{\|} \mathbf{d} \bullet{ }_{s \mathbf{D}^{\star}}^{\|} \mathbf{d}: V e c_{\star_{s}} \rightarrow V e c_{\star_{s}} \otimes_{\star_{s}} \Omega_{\star}^{2},
$$

which is $\mathcal{A}_{s}^{\star}$-linear and determined by a star s-connection ${ }_{s}^{\| \prime} \mathbf{D}^{\star}$ (A.1). A ${ }_{s}^{\|} \Re^{\star}$ is also $U \operatorname{Vec} c^{\mathcal{F}}\left(\mathcal{M},{ }_{s} N\right)$ equivariant and can be written in terms of $\mathrm{N}$-adapted associative composition $\bullet$, pairing and braiding $\tau_{\mathcal{R}}$ (A.6), and associator $\Phi$,

$$
\begin{aligned}
{ }_{s} \Re^{\star}:= & \langle,\rangle_{\star_{a}} \bullet\left({ }_{s}{ }_{s} \mathbf{D}^{\star} \otimes_{\star_{s}} i d\right) \bullet\left(\langle,\rangle_{\star_{s}} \otimes_{\star_{s}} i d\right) \bullet \Phi_{V e c_{\star_{s}} \otimes_{\star_{s}} \Omega_{\star}^{1}, V e c_{\star_{s}}, V e c_{\star_{s}}}^{-1} \bullet\left({ }_{s} \mathbf{D}^{\star} \otimes_{\star_{s}} i d^{\otimes_{\star_{s}}^{2}}\right) \\
& \bullet\left(i d^{\otimes_{\star_{s}}^{3}}-i d \otimes_{\mathcal{R}} \tau_{\mathcal{R}}\right)+\langle,\rangle_{\star_{s}} \bullet\left({ }^{\star} \mathbf{D}^{\star} \otimes_{\star_{s}} i d\right) \bullet\left(i d \otimes_{\mathcal{R}}[,]_{\star_{s}}\right) .
\end{aligned}
$$

Using formulas (A.2), the action of s-operator ${ }_{s}^{\prime \prime} \Re^{\star}\left(\right.$ A.9) on " $\mathbf{e}_{\alpha_{s}}$ can be computed and expressed in the form

$$
{ }_{s}^{" 1} \Re^{\star}\left(" \mathbf{e}_{\alpha_{s}}\right)={ }_{s \mathbf{D}^{\star}}^{\|} \mathbf{d}\left({ }_{s} \mathbf{D}^{\star} " \mathbf{e}_{\alpha_{s}}\right)={ }_{s \mathbf{D}^{\star}} \mathbf{d}\left(" \mathbf{e}_{\beta_{s}} \otimes_{\star_{s}} " \boldsymbol{\Gamma}_{\star \alpha_{s}}^{\beta_{s}}\right)=" \mathbf{e}_{\gamma_{s}} \otimes_{\star_{s}}{ }^{"} \mathcal{R}^{\star \gamma_{s}}{ }_{\alpha_{s}},
$$

where the the matrix valued describing a Hopf s-algebra star deformed s-curvature 2-form is

$$
" \Re^{\star \gamma_{s}}:={ }_{s} \mathbf{d} " \Gamma_{\star \alpha_{s}}^{\gamma_{s}}+{ }^{"} \boldsymbol{\Gamma}_{\star \beta_{s}}^{\gamma_{s}} \wedge_{\star_{s}} " \boldsymbol{\Gamma}_{\star \alpha_{s}}^{\beta_{s}} .
$$




\section{Coefficient formulas for the nonassociative Riemann s-tensor and quasi-Hopf s-algebras:}

Such formulas are expressed for the star deformed curvature s-tensor of ${ }_{s}^{\|} \mathbf{D}^{\star}$, i.e. for ${ }_{s}^{\|} \Re^{\star \gamma_{s}} \alpha_{s}$ (A.11), with respect to " $\mathbf{e}_{\alpha_{s}}$ (5),

$$
\begin{aligned}
& { }_{s}^{\|} \Re^{\star} \quad\left(" \mathbf{e}_{\alpha_{s}}, " \mathbf{e}_{\beta_{s}}, " \mathbf{e}_{\gamma_{s}}\right)=\left\langle " \mathbf{e}_{\mu_{s}} \otimes_{\star_{s}} \quad \stackrel{\mathrm{s}}{\| \Re^{\star \mu_{s}}}{ }_{\alpha_{s}}, " \mathbf{e}_{\beta_{s}} \wedge_{\star_{\mathbf{s}}} " \mathbf{e}_{\gamma_{s}}\right\rangle_{\star_{s}} \\
& =" \mathbf{e}_{\mu_{s} \star_{s}}\left\langle\text { "e } \mathbf{e}_{\nu_{s}} " \Gamma_{\star \alpha_{s} \varphi_{s} \star_{s}}^{\mu_{s}}\left(" \mathbf{e}^{\nu_{s}} \wedge_{\star_{s}} " \mathbf{e}^{\varphi_{s}}\right)+\right. \\
& \left." \boldsymbol{\Gamma}_{\star \nu_{s} \varphi_{s} \star_{s}}^{\mu_{s}}\left(\delta_{\tau_{s}}^{\varphi_{s}} " \boldsymbol{\Gamma}_{\star \alpha_{s} \lambda_{s}}^{\nu_{s}}+i \kappa \mathcal{R}_{\tau_{s}}^{\varphi_{s} \xi_{s}} " \mathbf{e}_{\xi_{s}} \boldsymbol{\Gamma}_{\star \alpha_{s} \lambda_{s}}^{\nu_{s}}\right) \star_{s} " \mathbf{e}^{\tau_{s}} \wedge_{\star_{\mathbf{s}}} " \mathbf{e}^{\lambda_{s}}, " \mathbf{e}_{\beta_{s}} \wedge_{\star_{\mathbf{s}}} " \mathbf{e}_{\gamma_{s}}\right\rangle_{\star_{s}} \\
& =" \mathbf{e}_{\mu_{s}} \star_{s} " \Re^{\star \mu_{s}}{ }_{\alpha_{s} \beta_{s} \gamma_{s}} \text {, }
\end{aligned}
$$

where $\delta_{\tau_{s}}^{\varphi_{s}}$ is the Kronecker delta symbol and the nonassociative Riemann s-tensor for the quasi-Hopf s-algebra can be written in the form

$$
\begin{aligned}
& " \Re_{\alpha_{s} \beta_{s} \gamma_{s}}^{\star \mu_{s}}=\mathbf{1}_{\alpha_{s} \beta_{s} \gamma_{s}}+\Re_{\mathbf{2}}^{\star \mu_{s}} \Re_{\alpha_{s} \beta_{s} \gamma_{s}}^{\star \mu_{s}} \text {, where } \\
& { }_{1} \Re_{\alpha_{s} \beta_{s} \gamma_{s}}^{\star \mu_{s}}=" \mathbf{e}_{\gamma_{s}} " \Gamma_{\star \alpha_{s} \beta_{s}}^{\mu_{s}}-" \mathbf{e}_{\beta_{s}} " \Gamma_{\star \alpha_{s} \gamma_{s}}^{\mu_{s}}+" \Gamma_{\star \nu_{s} \tau_{s} \star_{s}}^{\mu_{s}}\left(\delta_{\gamma_{s}}^{\tau_{s}} " \Gamma_{\star \alpha_{s} \beta_{s}}^{\nu_{s}}-\delta_{\beta_{s}}^{\tau_{\tau_{s}}} " \Gamma_{\star \alpha_{s} \gamma_{s}}^{\nu_{s}}\right) \\
& +" w_{\beta_{s} \gamma_{s}}^{\tau_{s}} \star_{s} " \Gamma_{\star \alpha_{s} \tau_{s}}^{\mu_{s}}
\end{aligned}
$$

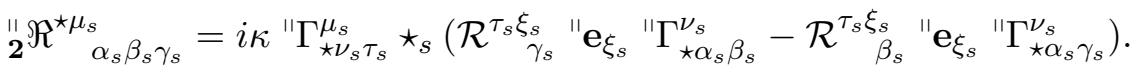

With respect to " $\mathbf{e}_{\alpha_{s}}$ (9) and for " $\boldsymbol{\Gamma}_{\star \alpha_{s} \beta_{s}}^{\gamma_{s}}$ (A.4), we find such a s-decomposition:

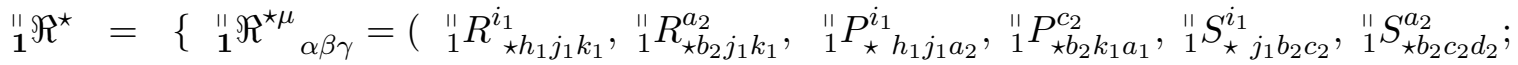

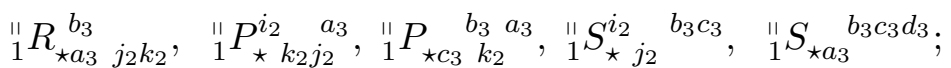

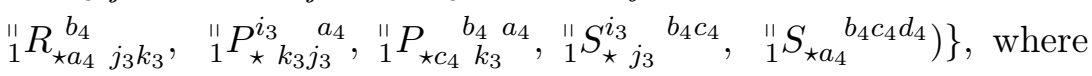

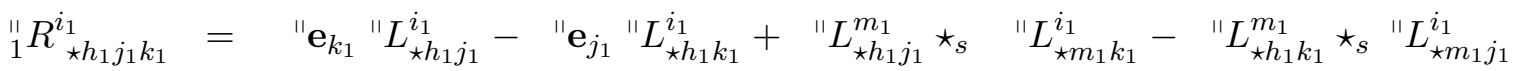

$$
\begin{aligned}
& -" C_{\star h_{1} a_{2}}^{i_{1}} \star_{s} " \Omega_{\star k_{1} j_{1}}^{a_{2}}, \\
& { }_{1}^{\|} R_{\star b_{2} j_{1} k_{1}}^{a_{2}}=" \mathbf{e}_{k_{1}} " \dot{L}_{\star b_{2} j_{1}}^{a_{2}}-" \mathbf{e}_{j_{1}} " \dot{L}_{\star b_{2} k_{1}}^{a_{2}}+"{ }^{a_{\star c_{2} j_{1}} \star_{s}}{ }^{a_{2}} \dot{L}_{\star b_{2} k_{1}}^{c_{2}}-"{ }^{a_{\star c_{2} k_{1}} \star_{s}} "{ }^{a_{2}} L_{\star b_{2} j_{1}}^{c_{2}} \\
& -" C_{\star b_{2} c_{2}}^{a_{2}} \star_{s} " \Omega_{\star k_{1} j_{1}}^{c_{2}} \text {, } \\
& { }_{1} P_{\star h_{1} j_{1} a_{2}}^{i_{1}}={ }^{\prime} e_{a_{2}} " L_{\star j_{1} k_{1}}^{i_{1}}-{ }^{i_{1}} D_{k_{1}}^{\star} \star_{s} " C_{\star j_{1} a_{2}}^{i_{1}}+{ }^{i_{1}} C_{\star j_{1} b_{2}}^{i_{1} \star_{s}} " T_{\star k_{1} a_{2}}^{b_{2}}, \\
& { }_{1} P_{\star b_{2} k_{1} a_{1}}^{c_{2}}={ }^{2} e_{a_{2}} " L_{\star b_{2} k_{1}}^{c_{2}}-{ }^{c_{2}} D_{k_{1}}^{\star} \star_{s} " C_{\star b_{2} a_{2}}^{c_{2}}+{ }^{\prime \prime} C_{\star b_{2} d_{2}}^{c_{2}} \star_{s} " T_{\star k_{1} a_{2}}^{d_{2}},
\end{aligned}
$$

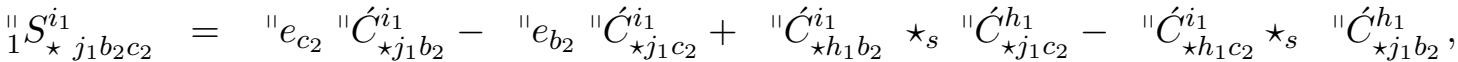

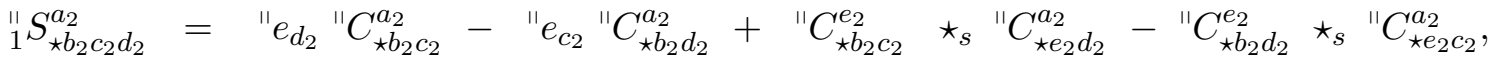

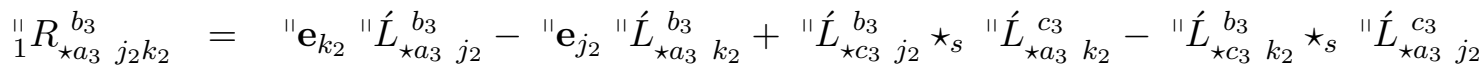

$$
\begin{aligned}
& -" C_{\star a_{3}}^{b_{3} c_{3}} \star_{s} " \Omega_{\star c_{3} k_{2} j_{2}},
\end{aligned}
$$

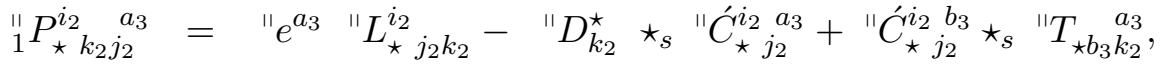

$$
\begin{aligned}
& { }_{1}^{\|} P_{\star c_{3} k_{2}} b_{3} a_{3}=" e^{a_{3}} " L_{\star c_{3} k_{2}}^{b_{3}}-" D_{k_{2}}^{\star} \star_{s} " C_{\star c_{3}} b_{3} a_{3}+" C_{\star c_{3}} b_{3} d_{3} \star_{s} " T_{\star d_{3} k_{2}} a_{3},
\end{aligned}
$$

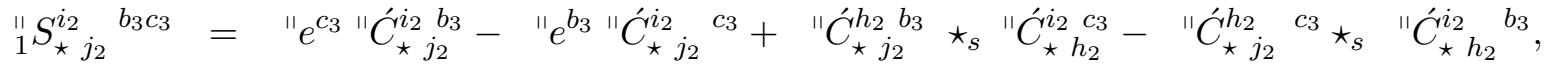

$$
\begin{aligned}
& { }_{1}^{1} S_{\star a_{3}} b_{3} c_{3} d_{3}=" e^{d_{3}} " C_{\star a_{3}} b_{3} c_{3}-" e^{c_{3}} " C_{\star a_{3}} b_{3} d_{3}+" C_{\star e_{3}} b_{3} c_{3} \star_{s} " C_{\star a_{3}} e_{3} d_{3}-" C_{\star e_{3}} b_{3} d_{3} \star_{s}{ }^{"} C_{\star a_{3}} e_{3} c_{3},
\end{aligned}
$$




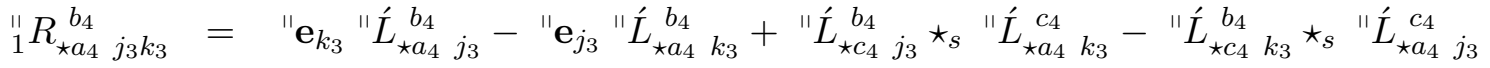

$$
\begin{aligned}
& -" C_{\star a_{4}}^{b_{4} c_{4}} \star_{s} " \Omega_{\star c_{4} k_{3} j_{3}} \text {, }
\end{aligned}
$$

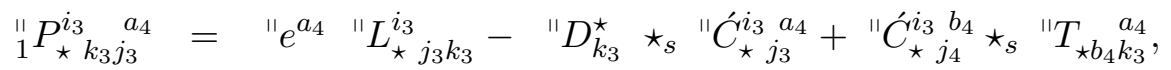

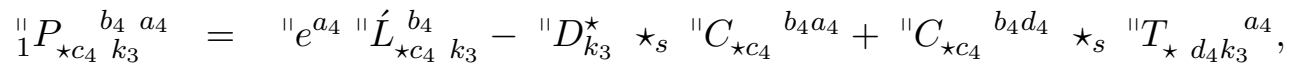

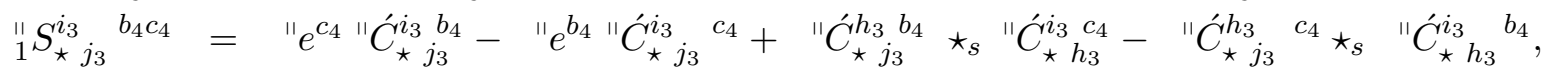

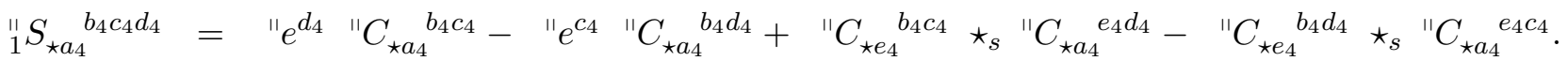

Tedious computations when (9) and (A.4) are used for ${ }_{2}^{11} R^{\star \mu}{ }_{\alpha \beta \gamma}$ allow us to compute s-coefficients in explicit form (we omit such formulas because they are not used in this paper).

We emphasize that the nonassociative Riemann s-tensor (A.13) is constructed for an arbitrary star deformed s-connection " $\mathbf{D}^{\star}$ (A.1), i.e. we have a dyadic model of nonassociative nonholonomic geometry of affine (linear) connections adapted to quasi-Hopf s-structures.

\section{The star deformed Ricci s-tensor for quasi-Hopf s-adapted structures:}

Contracting the first and forth indices in the Riemann s-tensor formulas ${ }_{s} \Re^{\star}$ (A.9), (A.10), and/or (A.12), we can define and compute a respective Ricci s-tensor. Such Riemann and Ricci s-tensors consist respective dyadic variants of " $\Re^{\star}$ and $" \Re i c^{\star}$ introduced in subsections of 3.1 and 3.2 of [3]. Here we nota that similar $\mathrm{N}$-and s-structures were studied for nonholonomic and noncommutative models elaborated in our previous works [17, 18, 41, see also parts II and III of monograph [44. Using star curvature s-operator (A.10), we define

$$
{ }_{s}^{"} \Re i c^{\star}(" \mathbf{z}, " \mathbf{v}):=\left\langle{ }_{s}^{"} \Re^{\star}\left({ }^{~ "} \mathbf{z}, " \mathbf{v}, " \mathbf{e}_{\alpha_{s}}\right), " \mathrm{e}^{\alpha_{s}}\right\rangle_{\star_{s}},
$$

for any s-vectors "z $\rightarrow{ }_{s}{ }_{s} \mathbf{z}, " v \rightarrow{ }_{s} " v \in V e c_{\star_{s}}$ and s-adapted (co) frames " $\mathbf{e}_{\alpha_{s}}$ and $" \mathbf{e}^{\beta_{s}}$.

The star Ricci s-tensor is defined by following such formulas

$$
\begin{aligned}
& { }_{s}^{"} \Re i c^{\star}=" \mathbf{R} i c_{\alpha_{s} \beta_{s}}^{\star} \star_{s}\left(" \mathbf{e}^{\alpha_{s}} \otimes_{\star_{s}} " \mathbf{e}^{\beta_{s}}\right) \text {, where } \\
& \text { "R } i c_{\alpha_{s} \beta_{s}}^{\star}:={ }_{s} \Re i c^{\star}\left("{ }_{\alpha_{s}}, " \mathbf{e}_{\beta_{s}}\right)=\left\langle " \mathbf{R} i c_{\mu_{s} \nu_{s}}^{\star} \star_{s}\left(" \mathbf{e}^{\mu_{s}} \otimes_{\star_{s}} "{ }^{\nu_{s}}\right), \quad " \mathbf{e}_{\alpha_{s}} \otimes_{\star_{s}} " \mathbf{e}_{\beta_{s}}\right\rangle_{\star_{s}} \text {. }
\end{aligned}
$$

Using s-coefficient formulas for $" \Re^{\star}\left(" \mathbf{e}_{\alpha}, " \mathbf{e}_{\beta}, " \mathbf{e}_{\gamma}\right)($ A.12) and the property that the associator acts trivially on basis s-vectors and s-adapted forms (and contracting respective indices in (A.13) and (A.14)), we can calculate explicitly all s-adapted coefficients

$$
\begin{aligned}
& " \mathbf{R} i c_{\alpha_{s} \beta_{s}}^{\star}={ }^{\|} \Re_{\alpha_{s} \beta_{s} \mu_{s}}^{\star \mu_{s}}={ }_{\mathbf{1}}^{11} \mathbf{R} i c_{\alpha_{s} \beta_{s}}^{\star}+{ }_{2}^{" 1} \mathbf{R} i c_{\alpha_{s} \beta_{s}}^{\star} \text {, for } \\
& { }_{1}{ }_{1} \mathbf{R} i c_{\alpha_{s} \beta_{s}}^{\star}={ }_{1}^{\|} \Re_{\alpha_{s} \beta_{s} \mu_{s}}^{\star \mu_{s}} \text { and }{ }_{2}^{"} \mathbf{R} i c_{\alpha_{s} \beta_{s}}^{\star}={ }_{2} \Re_{\alpha_{s} \beta_{s} \mu_{s}}^{\star \mu_{s}} \text {. }
\end{aligned}
$$

Such computations are presented in details via associative and commutative formulas in (A1) of [4, 5]), see also references therein. In dyadic form, we obtain such s-coefficients:

$$
\begin{aligned}
& \text { "R } i c_{\alpha_{s} \beta_{s}}^{\star}=\left\{" R_{\star h_{1} j_{1}}=" \Re^{i_{1}}{ }_{\star h_{1} j_{1} i_{1}}, \quad " P_{\star j_{1} a_{2}}=-"{ }^{i_{\star}} \Re_{\star j_{1} i_{1} a_{2}}, " P_{\star b_{2} k_{1}}="{ }^{i_{\star}} \Re_{\star b_{2} k_{1} c_{2}}^{c_{2}}, " S_{\star b_{2} c_{2}}=" \Re_{\star b_{2} c_{2} a_{2}}^{a_{2}},\right. \\
& " P_{\star j_{2}}^{a_{3}}=-" \Re_{\star j_{2} i_{2}}^{i_{2}} a_{3}, " P_{\star k_{2}}^{b_{3}}=" \Re_{\star c_{3} k_{2}}^{b_{3} c_{3}}, " S_{\star}^{b_{3} c_{3}}=" \Re_{\star a_{3}} b_{3} c_{3} a_{3}, \\
& \left." P_{\star j_{3}}^{a_{4}}=-" \Re_{\star j_{3} i_{3}}^{i_{3} a_{4}}, " P_{\star k_{3}}^{b_{4}}=" \Re_{\star c_{4} k_{3}}^{b_{4} c_{4}}, " S_{\star}^{b_{4} c_{4}}=" \Re_{\star a_{4}} b_{4} c_{4} a_{4}\right\} .
\end{aligned}
$$

We can decompose this s-tensor in powers of $i \kappa$,

$$
{ }^{\|} \mathbf{R} i c_{\alpha_{s} \beta_{s}}^{\star}=(i \kappa)^{0}{ }_{[0]}^{\|} \mathbf{R} i c_{\alpha_{s} \beta_{s}}^{\star}+(i \kappa)^{1}{ }_{[1]}^{\|} \mathbf{R} i c_{\alpha_{s} \beta_{s}}^{\star}-\kappa^{2}{ }_{[2]}^{\|} \mathbf{R} i c_{\alpha_{s} \beta_{s}}^{\star},
$$

when ${ }_{[0]}^{11} \mathbf{R} i c_{\alpha \beta}^{\star}$ include nonassociative and noncommutative contributions from star products of type (10) and their generalizations for quasi-Hopf s-structures. These decompositions are similar to formulas (4.83) 
in 2 . providing a holonomic version of such a decomposition on powers of $\kappa$. In this work, we generalize in nonhollonomic dyadic form those formulas considering that $\partial \rightarrow{ }_{s} \mathbf{e}$ and nontrivial s-connection structure result in non-zero anholonomy coefficients " $w_{\alpha_{s} \gamma_{s}}^{\nu_{s}}$. Such nonholonomic s-structures allow us to apply the AFCDM.

\section{A.3 Nonassociative s-metrics and canonical s-connections with small parameters}

Decompositions of star nonholonomic deformations of d-metrics and canonical d-connections on small parameters $\hbar$ and $\kappa$ were studied in section 3.3.2 of [3]. Here, we extended in N-adapted form the coordinate frame approach to nonassociative geometry with quasi-Hopf structures, (non) symmetric metrics and LCconnections with parametric decompositions provided in section 5.3 of [2].

We can transform all (non) commutative and/ or nonassociative geometric d-objects into s-objects (we shall omit dubbing coordinate formulas from just cited works) using nonholonomic dyadic decompositions with two linear star deformed connection structures (34), s-frames and (non) symmetric metrics and respective canonical s-distortions (41) determined by fundamental geometric data:

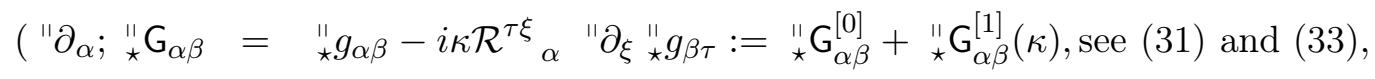

$$
\begin{aligned}
& { }_{\star}^{"} \mathrm{G}^{\alpha \beta}=" g^{\alpha \beta}-i \kappa " g^{\alpha \tau} \mathcal{R}_{\tau}^{\mu \nu}\left(" \partial_{\mu} " g_{\nu \varepsilon}\right) " g^{\varepsilon \beta}+O\left(\kappa^{2}\right):={ }_{\star} \mathrm{G}_{[0]}^{\alpha \beta}+{ }_{\star} \mathrm{G}_{[1]}^{\alpha \beta}(\kappa)+O\left(\kappa^{2}\right) ; \\
& \left." \nabla^{\star}, \text { see (34) }\right) \\
& \mathbb{1}
\end{aligned}
$$

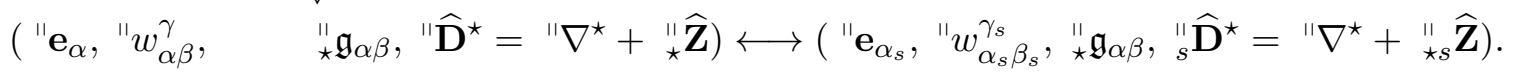

Such transforms allow to compute additionally to holonomic geometric objects with " $\partial_{\alpha}$ certain new star nonholonomic terms of the d-/ s-curvature and d-/s-torsion, and Ricci d-/ s-tensor. The nonholonomic star distortions appear, for instance, with s-adapted frames $" \mathbf{e}_{\alpha_{s}}$ and their nontrivial anholonomy coefficients " $w_{\alpha_{s} \beta_{s}}^{\gamma_{s}}$.

We provide in this subsection certain important s-formulas which show how the nonassociative geometric dyadic formalism differs from the cases with (non) holonomic and N-connection structures. There will be introduced certain parametric on $\hbar$ and $\kappa$ formulas written in generic off-diagonal and/or s-adapted forms.

Let us introduce in a coordinate local basis

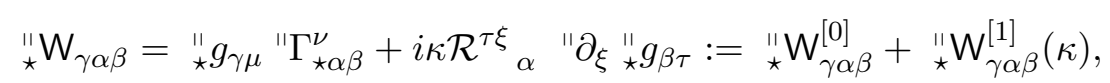

where, for respective zero parameters, " $\Gamma_{\star \alpha \beta \mid \hbar, \kappa=0}^{\nu}=" \Gamma_{\alpha \beta}^{\nu}$ are taken as the coefficients of the commutative LCconnection " $\nabla$ in (21). For nontrivial $\hbar$ and $\kappa$, we work with a nonassociative " $\nabla^{\star}$. The geometric techniques of decomposition on small parameters and (A.19) can be extended for nonholonomic dyadic canonical data $\left({ }_{\star} \mathfrak{g}_{\alpha_{s} \beta_{s}},{ }_{s}^{\prime \prime} \widehat{\mathbf{D}}^{\star}\right)$ following Convention 2 (29) and rules (A.18), working in s-adapted (co) bases. We express

$$
\begin{aligned}
& { }_{\star}^{\|} \mathfrak{g}_{\alpha_{s} \beta_{s}}={ }_{\star} " \mathbf{g}_{\alpha_{s} \beta_{s}}-i \kappa \overline{\mathcal{R}}^{\tau_{s} \xi_{s}}{ }_{\alpha_{s}} " \mathbf{e}_{\xi_{s}}{ }_{\star} \mathbf{g}_{\beta_{s} \tau_{s}}:={ }_{\star} " \mathfrak{g}_{\alpha_{s} \beta_{s}}^{[0]}+{ }_{\star} " \mathfrak{g}_{\alpha_{s} \beta_{s}}^{[1]}(\kappa) \text { and }
\end{aligned}
$$

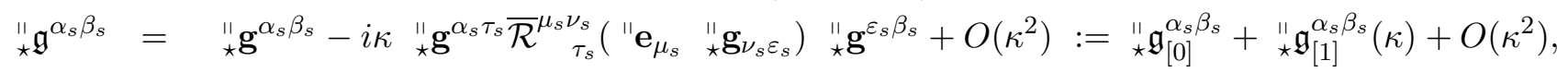

$$
\begin{aligned}
& { }_{*}^{\|} \mathfrak{g}^{\alpha_{s} \beta_{s}}=2{ }_{\star}^{\|} \mathfrak{g}^{\alpha_{s} \beta_{s}}-{ }_{\star}^{\|} \mathfrak{g}^{\alpha_{s} \gamma_{s}} *{ }_{\star}^{\|} \mathfrak{g}_{\gamma_{s} \tau_{s}} *{ }_{\star} \mathfrak{g}^{\tau_{s} \beta_{s}}+O\left(\hbar^{2}\right),
\end{aligned}
$$

with possible explicit s-splitting of the star s-metric ${ }_{\star}^{\|} \mathfrak{g}_{\gamma_{s} \tau_{s}}$ (32) into h1-v2-c3-c4 components for respective shells $s=1,2,3,4$. For a star canonical s-connection " $\widehat{\Gamma}_{\star \alpha_{s} \beta_{s}}^{\nu_{s}}$ (40), we consider similarly to (A.19) that

$$
{ }_{\star}^{" \prime} \widehat{W}_{\gamma_{s} \alpha_{s} \beta_{s}}={ }_{\star}{ }_{\star} \mathbf{g}_{\gamma_{s} \mu_{s}} " \widehat{\Gamma}_{\star \alpha_{s} \beta_{s}}^{\nu_{s}}+i \kappa \overline{\mathcal{R}}^{\tau_{s} \xi_{s}} \alpha_{s} " \mathbf{e}_{\xi_{s}}{ }_{\star} \mathbf{g}_{\beta_{s} \tau_{s}}:={ }_{\star}{ }_{\star} \widehat{W}_{\gamma_{s} \alpha_{s} \beta_{s}}^{[0]}+{ }_{\star}{ }_{\star} \widehat{W}_{\gamma_{s} \alpha_{s} \beta_{s}}^{[1]}(\kappa) .
$$


We can re-define the $\mathrm{N}$-adapted formulas to s-adapted one 8 with coefficients up to parameters $\hbar, \kappa$ and $\hbar \kappa$ for the star s-metrics (symmetric and nonsymmetric) and star canonical s-connections. The coordinate formulas for the LC-connections can be distorted following (41).

\section{A.3.1 Parametric decomposition of inverse nonsymmetric star s-metrics}

Considering in explicit form the s-coefficients proportional to both parameters, we obtain such formulas for the nonsymmetric d-metric from (A.20):

$$
\begin{aligned}
& { }_{*}^{\prime \prime} \mathfrak{g}_{[0]}^{\alpha_{s} \beta_{s}}={ }_{\star}^{\|} \mathfrak{g}_{[00]}^{\alpha_{s} \beta_{s}}+{ }_{\star} \mathfrak{x}_{[01]}^{\alpha_{s} \beta_{s}}(\hbar)+O\left(\hbar^{2}, \kappa^{2}\right) \text { and }{ }_{*}^{\prime \prime} \mathfrak{g}_{[1]}^{\alpha \beta}={ }_{\star} \mathfrak{x}_{[10]}^{\alpha \beta}(\kappa)+{ }_{\star}^{\prime \prime} \mathfrak{g}_{[11]}^{\alpha \beta}(\hbar \kappa)+O\left(\hbar^{2}, \kappa^{2}\right), \\
& \text { where }{ }_{\star}^{\|} \mathfrak{g}_{[00]}^{\alpha_{s} \beta_{s}}=" \mathrm{~g}^{\alpha_{s} \beta_{s}} \text {, } \\
& { }_{\star} \mathfrak{g}_{[01]}^{\alpha_{s} \beta_{s}}=\frac{i \hbar}{2}\left(" \mathbf{e}^{n+i_{1}} " \mathbf{g}^{\alpha_{s} \gamma_{s}} " \mathbf{e}_{i_{1}} " \mathbf{g}_{\gamma_{s} \tau_{s}}-" \mathbf{e}_{i_{1}} " \mathbf{g}^{\alpha_{s} \gamma_{s} "} \mathbf{e}^{n+i_{1}} " \mathbf{g}_{\gamma_{s} \tau_{s}}+\right. \\
& \left." \partial^{n+i_{2}} " \mathbf{g}^{\alpha_{s} \gamma_{s}} \quad " \mathbf{e}_{i_{2}} " \mathbf{g}_{\gamma_{s} \tau_{s}}-" \mathbf{e}_{i_{2}} " \mathbf{g}^{\alpha_{s} \gamma_{s}} " \partial^{n+i_{2}} " \mathbf{g}_{\gamma_{s} \tau_{s}}\right) " \mathbf{g}^{\tau_{s} \beta_{s}}, \\
& { }_{\star}^{\prime \prime} \mathfrak{g}_{[10]}^{\alpha_{s} \beta_{s}}=i \kappa \overline{\mathcal{R}}^{\tau_{s} \xi_{s}} \gamma_{s} " \mathbf{g}^{\alpha_{s} \gamma_{s}}\left(" \mathbf{e}_{\xi_{s}} " \mathbf{g}_{\mu_{s} \tau_{s}}\right) " \mathbf{g}^{\mu_{s} \beta_{s}}, \\
& { }_{\star}{ }_{\star} \mathfrak{g}_{[11]}^{\alpha_{1} \beta_{1}}=\frac{\hbar \kappa}{2} \overline{\mathcal{R}}_{\gamma_{1}}^{\tau_{1} \xi_{1}}\left\{( " \mathbf { e } _ { \xi _ { 1 } } " \mathbf { g } _ { \mu _ { 1 } \tau _ { 1 } } ) \left[" \mathbf { g } ^ { \nu _ { s } \gamma _ { s } } " \mathbf { g } ^ { \mu _ { s } \beta _ { 1 } } \left(" \mathbf{e}_{i_{1}} " \mathbf{g}^{\alpha_{s} \lambda_{s}} " \mathbf{e}^{n+i_{1}} " \mathbf{g}_{\lambda_{s} \nu_{s}}-\right.\right.\right.
\end{aligned}
$$

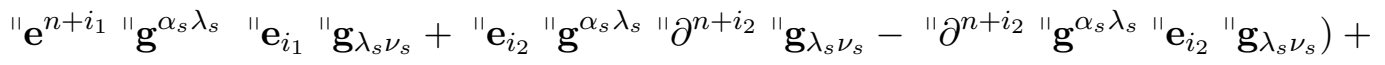

$$
\begin{aligned}
& \text { " } \mathbf{g}^{\alpha_{s} \gamma_{s} "} \mathbf{g}^{\nu_{s} \beta_{s}}\left(" \mathbf{e}_{i_{1}} " \mathbf{g}^{\mu_{s} \lambda_{s}} " \mathbf{e}^{n+i_{1}} " \mathbf{g}_{\lambda_{s} \nu_{s}}-" \mathbf{e}^{n+i_{1}} " \mathbf{g}^{\mu_{s} \lambda_{s}} " \mathbf{e}_{i_{1}} " \mathbf{g}_{\lambda_{s} \nu_{s}}+\right. \\
& \text { " } \left.\mathbf{e}_{i_{2}} " \mathbf{g}^{\mu_{s} \lambda_{s} "} \partial^{n+i_{2}} " \mathbf{g}_{\lambda_{2} \nu_{2}}-" \partial^{n+i_{2}} " \mathbf{g}^{\mu_{s} \lambda_{s}} " \mathbf{e}_{i_{2}} " \mathbf{g}_{\lambda_{s} \nu_{s}}\right)+\left(" \mathbf{e}_{i_{1}} " \mathbf{g}^{\alpha_{s} \gamma_{s}} " \mathbf{e}^{n+i_{1}} " \mathbf{g}^{\mu_{s} \beta_{s}}-\right. \\
& \text { "e } \left.\left.\mathbf{e}^{n+i_{1}} " \mathbf{g}^{\alpha_{s} \gamma_{s}} " \mathbf{e}_{i_{1}} " \mathbf{g}^{\mu_{s} \beta_{s}}+" \mathbf{e}_{i_{2}} " \mathbf{g}^{\alpha_{s} \gamma_{s} "} \partial^{n+i_{2}} " \mathbf{g}^{\mu_{s} \beta_{s}}-" \partial^{n+i_{2}} " \mathbf{g}^{\alpha_{s} \gamma_{s}} " \mathbf{e}_{i_{2}} " \mathbf{g}^{\mu_{s} \beta_{s}}\right)\right]- \\
& \left(\text { ( } \mathbf{e}_{i_{1}} " \mathbf{e}_{\xi_{s}} " \mathbf{g}_{\nu_{s} \tau_{s}}\right)\left({ }^{\prime \prime} \mathbf{g}^{\alpha_{s} \gamma_{s}} " \mathbf{e}^{n+i_{1}} " \mathbf{g}^{\nu_{s} \beta_{s}}-{ }^{\prime} \mathbf{g}^{\nu_{s} \beta_{s}} " \mathbf{e}^{n+i_{1}} " \mathbf{g}^{\alpha_{s} \gamma_{s}}\right)- \\
& \left({ }^{~ "} \mathbf{e}_{i_{2}} " \mathbf{e}_{\xi_{s}} " \mathbf{g}_{\nu_{s} \tau_{s}}\right)\left({ }^{n} \mathbf{g}^{\alpha_{s} \gamma_{s}} " \partial^{n+i_{2}} " \mathbf{g}^{\nu_{s} \beta_{s}}-{ }^{\prime \prime} \mathbf{g}^{\nu_{s} \beta_{s}} " \partial^{n+i_{2}} " \mathbf{g}^{\alpha_{s} \gamma_{s}}\right)+ \\
& \left(\text { ( } \mathbf{e}^{n+i_{1}} " \mathbf{e}_{\xi_{s}} " \mathbf{g}_{\nu_{s} \tau_{s}}\right)\left(" \mathbf{g}^{\alpha_{s} \gamma_{s}} " \mathbf{e}_{i_{1}} " \mathbf{g}^{\nu_{s} \beta_{s}}-" \mathbf{g}^{\nu_{s} \beta_{s}} " \mathbf{e}_{i_{1}} " \mathbf{g}^{\alpha_{s} \gamma_{s}}\right)+ \\
& \left.\left(" \partial^{n+i_{2}} " \mathbf{e}_{\xi_{s}} " \mathbf{g}_{\nu_{s} \tau_{s}}\right)\left(" \mathbf{g}^{\alpha_{s} \gamma_{s}} " \mathbf{e}_{i_{2}} " \mathbf{g}^{\nu_{s} \beta_{s}}-" \mathbf{g}^{\nu_{s} \beta_{s}} " \mathbf{e}_{i_{2}} " \mathbf{g}^{\alpha_{s} \gamma_{s}}\right)\right\}+O\left(\hbar^{2}, \kappa^{2}\right) \text {, } \\
& \text { for " } \mathrm{e}^{n+i_{1}}=\frac{\partial}{\partial " p_{n+i_{1}}}+{ } N_{n+i_{2}}^{n+i_{1}} \frac{\partial}{\partial " p_{n+i_{2}}}, " \partial^{n+i_{2}}=\frac{\partial}{\partial " p_{n+i_{2}}} \text {, when } i_{1}=1,2 \text { and } i_{2}=3,4 \text {. }
\end{aligned}
$$

We can prescribe the nonholonomic s-frame structure when for " $\mathbf{e}_{\alpha_{s}} \rightarrow$ " $\partial_{\alpha}$ and, inversely, following the Convention 2 (29), all above formulas are equivalent correspondingly to coordinate base formulas (5.59) (5.61) computed in [2], see also N-adapted formulas (118) in [3].

\section{A.3.2 Nonholonomic dyadic parametric decomposition of star canonical s-connections}

Computing s-adapted parametric decompositions for ${ }_{\star}^{\prime \prime} \mathfrak{g}_{\alpha_{s} \beta_{s}}={ }_{\star} \mathfrak{x}_{\alpha_{s} \beta_{s}}^{[0]}+{ }_{\star}^{\prime \prime} \mathfrak{g}_{\alpha_{s} \beta_{s}}^{[1]},{ }_{*}^{\prime \prime} \mathfrak{g}^{\alpha_{s} \beta_{s}}={ }_{*} \mathfrak{*}_{[0]}^{\alpha_{s} \beta_{s}}+{ }_{*}^{\prime \prime} \mathfrak{g}_{[1]}^{\alpha_{s} \beta_{s}}$ (A.21) and " $\widehat{\boldsymbol{\Gamma}}_{\star \alpha_{s} \beta_{s}}^{\gamma_{s}}(\underline{40)}$, we obtain

$$
\begin{aligned}
& { }_{[0]} \widehat{\Gamma}_{* \alpha_{s} \beta_{s}}^{\nu_{s}}={ }_{*}^{\|} \mathfrak{g}_{[0]}^{\nu_{s} \gamma_{s}} *{ }_{*}{ }_{*} \widehat{W}_{\gamma_{s} \alpha \beta}^{[0]}:={ }_{[00]}^{\|} \widehat{\Gamma}_{\star \alpha \beta}^{\nu_{s}}+{ }_{[01]}^{\|} \widehat{\Gamma}_{\star \alpha \beta}^{\nu_{s}}(\hbar)+O\left(\hbar^{2}\right), \\
& { }_{[1]}^{\|} \widehat{\Gamma}_{* \alpha_{s} \beta_{s}}^{\nu_{s}}={ }_{*}^{\|} \mathfrak{g}_{[0]}^{\nu_{s} \gamma_{s}} *{ }_{*}^{\|} \widehat{W}_{\gamma_{s} \alpha_{s} \beta_{s}}^{[1]}+{ }_{*}{ }_{*} \mathfrak{g}_{[1]}^{\nu_{s} \gamma_{s}} *{ }_{*} " \widehat{W}_{\gamma_{s} \alpha_{s} \beta_{s}}^{[0]}:={ }_{[10]}^{\|} \widehat{\Gamma}_{\star \alpha_{s} \beta_{s}}^{\nu_{s}}(\kappa)+{ }_{[11]}^{\|} \widehat{\Gamma}_{\star \alpha_{s} \beta_{s}}^{\nu_{s}}(\hbar \kappa)+O\left(\hbar^{2}\right),
\end{aligned}
$$

\footnotetext{
${ }^{8}$ for holonomic configurations, see in details the Section 5.2, formulas (5.55)-(5.65), of [2] on decomposition of the coefficients of metrics and linear connections
} 


$$
\begin{aligned}
& \text { where }{ }_{[00]}^{\|} \widehat{\Gamma}_{\star \alpha_{s} \beta_{s}}^{\nu_{s}}=" \widehat{\Gamma}_{\alpha_{s} \beta_{s}}^{\nu_{s}},{ }_{[01]}^{\|} \widehat{\Gamma}_{\star \alpha_{s} \beta_{s}}^{\nu_{s}}=-\frac{i \hbar}{2} " \mathbf{g}^{\nu_{s} \gamma_{s}}\left(" \mathbf{e}_{i_{1}} " \mathbf{g}_{\gamma_{s} \tau_{s}} " \partial^{n+i_{1}} " \widehat{\Gamma}_{\alpha_{s} \beta_{s}}^{\tau_{s}}-\right.
\end{aligned}
$$

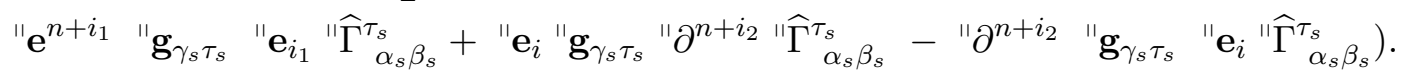

To simplify s-formulas with contracting of spacetime indices form $s=1,2$ with respective $s=3,4$, we shall use also such a convention:

$$
\text { "e } \mathbf{e}^{n+i_{s}}=\left(" \mathbf{e}^{n+i_{1}}=\frac{\partial}{\partial " p_{n+i_{1}}}+{ }^{\prime} N_{n+k_{2}}^{n+i_{1}} \frac{\partial}{\partial " p_{n+k_{2}}}, " \partial^{n+i_{2}}=\frac{\partial}{\partial " p_{n+i_{2}}}\right) \text {, when } i_{1}=1,2 \text { and } i_{2}=3,4 \text {. }
$$

So, we compute and write

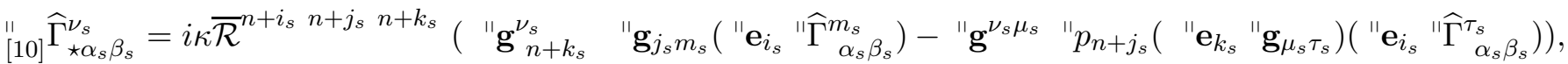

$$
\begin{aligned}
& { }_{[11]}^{\|} \widehat{\Gamma}_{\star \alpha_{s} \beta_{s}}^{\nu_{s}}=i \frac{\kappa \hbar}{2} \overline{\mathcal{R}}^{n+i_{s} n+j_{s} n+k_{s}}\left\{-\left(" \mathbf{e}_{l_{s}} " \mathbf{g}_{n+k_{s}}^{\nu_{s}}\right) " \mathbf{e}^{n+l_{s}}\left[" \mathbf{e}_{i_{s}}\left(" \mathbf{g}_{j_{s} m_{s}} " \widehat{\Gamma}_{\alpha_{s} \beta_{s}}^{m_{s}}\right)\right]+\right. \\
& \left(" \mathbf{e}^{n+l_{s}} " \mathbf{g}^{\nu_{s}}{ }_{n+k_{s}}\right)\left[" \mathbf{e}_{l_{s}}\left(" \mathbf{e}_{i_{s}} " \mathbf{g}_{j_{s} m_{s}} " \widehat{\Gamma}_{\alpha_{s} \beta_{s}}^{m_{s}}\right)\right]+ \\
& {\left[\left(" \mathbf{e}_{l_{s}} " \mathbf{g}^{\nu_{s} \gamma_{s}}\right)\left(\text { " } \mathbf{e}^{n+l_{s}} " \mathbf{g}_{\gamma_{s} \tau_{s}}\right)-\left({ }^{\prime \prime} \mathbf{e}^{n+l_{s}} " \mathbf{g}^{\nu_{s} \gamma_{s}}\right)\left({ }^{\prime \prime} \mathbf{e}_{l_{s}} " \mathbf{g}_{\gamma_{s} \tau_{s}}\right)\right] " \mathbf{g}_{n+k_{s}}^{\tau_{s}}\left(" \mathbf{e}_{i_{s}} " \mathbf{g}_{j_{s} m_{s}} " \widehat{\Gamma}^{m_{s}} \alpha_{s} \beta_{s}\right)+} \\
& \text { "| } e_{l_{s}}\left[{ }^{\prime} \mathbf{g}_{n+k_{s}}^{\nu_{s}} " \mathbf{g}^{q_{s} m_{s}}\left(" \mathbf{e}_{i_{s}} " \mathbf{g}_{q_{s} j_{s}}\right)\right] " \mathbf{e}^{n+l_{s}}\left(" \mathbf{g}_{m_{s} o_{s}} " \widehat{\Gamma}_{\alpha_{s} \beta_{s}}^{o_{s}}\right)- \\
& \text { "e } \mathbf{e}^{n+l_{s}}\left[\left(" \mathbf{g}_{n+k_{s}}^{\nu_{s}} " \mathbf{g}^{q_{s} m_{s}}\left(" \mathbf{e}_{i_{s}} " \mathbf{g}_{q_{s} j_{s}}\right)\right] " \mathbf{e}_{l_{s}}\left({ }^{\prime} \mathbf{g}_{m_{s} o_{s}} " \widehat{\Gamma}_{\alpha_{s} \beta_{s}}^{o_{s}}\right)-\right. \\
& \left({ }^{\prime \prime} \mathbf{e}_{i_{s}} " \mathbf{g}_{q_{s} j_{s}}\right)\left[{ }^{"} \mathbf{g}_{n+k_{s}}^{\tau_{s}}\left(\left(" \mathbf{e}_{l_{s}} " \mathbf{g}^{\nu_{s} \gamma_{s}}\right)\left(" \mathbf{e}^{n+l_{s}} " \mathbf{g}_{\gamma_{s} \tau_{s}}\right)-\left(" \mathbf{e}^{n+l_{s}} " \mathbf{g}^{\nu_{s} \gamma_{s}}\right)\left(" \mathbf{e}_{l_{s}} " \mathbf{g}_{\gamma_{s} \tau_{s}}\right)\right){ }^{"} \widehat{\Gamma}_{\alpha_{s} \beta_{s}}^{q_{s}}+\right. \\
& \text { " } \mathbf{g}_{n+k_{s}}^{\nu_{s}}\left(\left(" \mathbf{e}_{l_{s}} " \mathbf{g}^{\nu_{s} \gamma_{s}}\right)\left(" \mathbf{e}^{n+k_{s}} " \mathbf{g}_{\gamma_{s} \tau_{s}}\right)-\left(" \mathbf{e}^{n+k_{s}} " \mathbf{g}^{\nu_{s} \gamma_{s}}\right)\left(" \mathbf{e}_{l_{s}} " \mathbf{g}_{\gamma_{s} \tau_{s}}\right)\right) " \widehat{\Gamma}_{\alpha_{s} \beta_{s}}^{\tau_{s}}+
\end{aligned}
$$

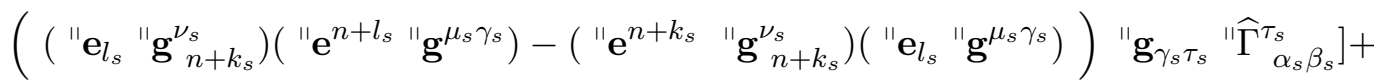

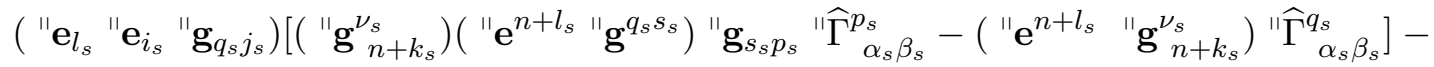

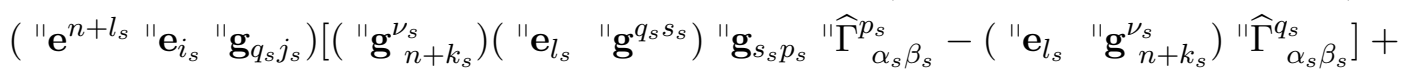

$$
\begin{aligned}
& p_{n+j_{s}}\left[" \mathbf{e}_{l_{s}}\left({ }^{\prime \prime} \mathbf{g}^{\nu_{s} \tau_{s}}\left({ }^{\prime \prime} \mathbf{e}_{k_{s}} " \mathbf{g}_{\tau_{s} \mu_{s}}\right)\right) " \mathbf{e}^{n+l_{s}}\left(\text { " } \mathbf{e}_{i_{s}} \quad \text { " } \widehat{\Gamma}_{\alpha_{s} \beta_{s}}^{\mu_{s}}\right)-\right.
\end{aligned}
$$

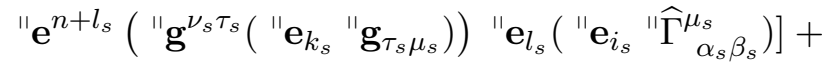

$$
\begin{aligned}
& p_{n+j_{s}} " \mathbf{g}^{\nu_{s} \tau_{s}}\left({ }^{\prime \prime} \mathbf{e}_{k_{s}} " \mathbf{g}_{\tau_{s} \mu_{s}}\right) " \mathbf{e}_{i_{s}}\left[\left(\left(" \mathbf{e}_{l_{s}} " \mathbf{g}^{\mu_{s} \gamma_{s}}\right) " \mathbf{e}^{n+l_{s}}\left("{ }^{\prime \prime} \boldsymbol{g}_{\gamma_{s} \tau_{s}} " \widehat{\Gamma}^{\tau_{s}}{ }_{\alpha_{s} \beta_{s}}\right)-\right.\right. \\
& \left.\left(\text { ( } \mathbf{e}^{n+l_{s}} " \mathbf{g}^{\mu_{s} \gamma_{s}}\right) " \mathbf{e}_{l_{s}}\left(" \mathbf{g}_{\gamma_{s} \tau_{s}} " \widehat{\Gamma}_{\alpha_{s} \beta_{s}}^{\tau_{s}}\right)\right)- \\
& \left.\left(\left({ }^{\prime} \mathbf{e}_{l_{s}} " \mathbf{g}^{\mu_{s} \gamma_{s}}\right)\left(" \mathbf{e}^{n+l_{s}} " \mathbf{g}_{\gamma_{s} \tau_{s}}\right)-\left(" \mathbf{e}^{n+l_{s}} " \mathbf{g}^{\mu_{s} \gamma_{s}}\right)\left(" \mathbf{e}_{l_{s}} " \mathbf{g}_{\gamma_{s} \tau_{s}}\right)\right) " \widehat{\Gamma}_{\alpha_{s} \beta_{s}}^{\tau_{s}}\right]-
\end{aligned}
$$

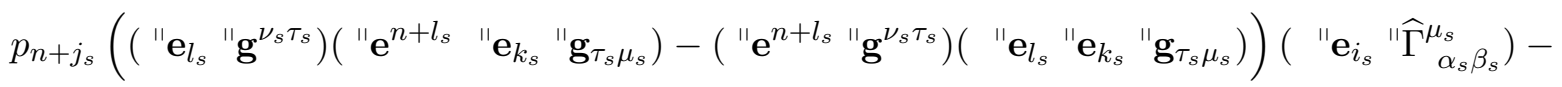

$$
\begin{aligned}
& p_{n+j_{s}}\left(\left(" \mathbf{e}_{l_{s}} " \mathbf{g}^{\nu_{s} \tau_{s}}\right)\left(" \mathbf{e}^{n+l_{s}} \quad \text { " } \mathbf{e}_{k_{s}} " \mathbf{g}_{\tau_{s} \varepsilon_{s}}\right)-\right. \\
& \left.\left(\text { " } \mathbf{e}^{n+l_{s}} " \mathbf{g}^{\nu_{s} \tau_{s}}\right)\left(\text { " } \mathbf{e}_{l_{s}} " \mathbf{g}_{\tau_{s} \varepsilon_{s}}\right)\right) " \mathbf{g}^{\varepsilon_{s} \lambda_{s}}\left(" \mathbf{e}_{k_{s}} " \mathbf{g}_{\lambda_{s} \mu_{s}}\right)\left(\text { " } \mathbf{e}_{i_{s}} " \widehat{\Gamma}_{\alpha_{s} \beta_{s}}^{\mu_{s}}\right)+ \\
& \left.\left({ }^{\prime \prime} \mathbf{e}_{l_{s}} " \mathbf{g}^{\nu_{s} \tau_{s}}\right)\left(" \mathbf{e}_{j_{s}} " \mathbf{g}_{\tau_{s} \mu_{s}}\right)\left(" \mathbf{e}_{k_{s}} " \widehat{\Gamma}_{\alpha_{s} \beta_{a}}^{\mu_{s}}\right)\right\} \text {. }
\end{aligned}
$$


These formulas are for the canonical s-connection " $\widehat{\mathbf{D}}^{\star}$ (41). We can extract formulas for the nonassociative LCconnection for zero distortion s-tensors when ${ }_{s}^{\prime \prime} \widehat{\mathbf{D}}_{\mid " \widehat{\mathbf{T}}=0}^{\star}=" \nabla^{\star}$, see (42). We can prescribe the nonholonomic s-frame structure in such a form when for " $\mathbf{e}_{\alpha_{s}} \rightarrow$ " $\partial_{\alpha}$ and, inversely, following the Convention 2 (29). This way we obtain both coordinate basis formulas (5.62) - (5.65) from [2] and formulas (119)-(120) from [3] if we redefine the constructions in $\mathrm{N}$-adapted form.

Above formulas can be subjected also to further to h1-v2-c3-c4-decompositions with respect to s-frames "e $\mathbf{e}^{\alpha_{s}}$ and " $\mathbf{e}_{\alpha_{s}}$, when ${ }_{[00]}^{\|} \widehat{\Gamma}_{* \alpha_{s} \beta_{s}}^{\nu_{s}}=" \widehat{\Gamma}_{\alpha_{s} \beta_{s}}^{\nu_{s}}$ are used for taking the canonical s-connection from (21) and then for considering R-flux star deformations of nonholonomic dyadic structure into nonassociative geometric models determined by nonassociative canonical s-connection from (34).

\section{A.3.3 Parametric coefficients for the nonassocative canonical Ricci s-tensor}

For the [00] term in (45), we compute

$$
\begin{aligned}
& { }_{[00]}^{\|} \widehat{\mathbf{R}} i c_{\beta_{s} \gamma_{s}}^{\star}={ }^{\prime \prime} \widehat{\mathbf{R}} i c_{\beta_{s} \gamma_{s}}=
\end{aligned}
$$

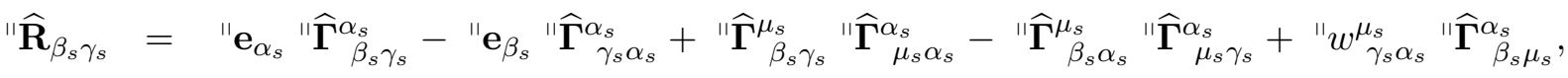

when further s-decompositions are stated by associative and commutative values (24).

The terms with labels $[0,1]$ determine the order $\hbar$ contribution to star deformations of the Ricci s-tensor for respective parametric decompositions of the canonical s-connection,

$$
\begin{aligned}
& { }_{[01]}^{\prime \prime} \widehat{\mathbf{R}} i c_{\beta_{s} \gamma_{s}}^{\star}=" \mathbf{e}_{\alpha_{s}}{ }_{[01]}^{\|} \widehat{\boldsymbol{\Gamma}}_{\beta_{s} \gamma_{s}}^{\alpha_{s}}-" \mathbf{e}_{\beta_{s}}{ }_{[01]}^{\|} \widehat{\boldsymbol{\Gamma}}_{\gamma_{s} \alpha_{s}}^{\alpha_{s}}+{ }_{[01]}^{\|} \widehat{\boldsymbol{\Gamma}}_{\beta_{s} \gamma_{s}}^{\mu_{s}} " \widehat{\boldsymbol{\Gamma}}_{\mu_{s} \alpha_{s}}^{\alpha_{s}}+" \widehat{\boldsymbol{\Gamma}}_{\beta_{s} \gamma_{s}}^{\mu_{s}}{ }_{[01]} \widehat{\boldsymbol{\Gamma}}_{\mu_{s} \alpha_{s}}^{\alpha_{s}} \\
& -{ }_{[01]} \widehat{\boldsymbol{\Gamma}}_{\beta_{s} \alpha_{s}}^{\mu_{s}} " \widehat{\boldsymbol{\Gamma}}_{\mu_{s} \gamma_{s}}^{\alpha_{s}}-" \widehat{\boldsymbol{\Gamma}}_{\beta_{s} \alpha_{s}[01]}^{\mu_{s}}{ }_{\mu_{\mu_{s} \gamma_{s}}}+\widehat{\boldsymbol{\Gamma}}^{\alpha_{s}} w_{\gamma_{s} \alpha_{s}[01]}^{\mu_{s}} \widehat{\boldsymbol{\Gamma}}_{\beta_{s} \mu_{s}}^{\alpha_{s}}
\end{aligned}
$$

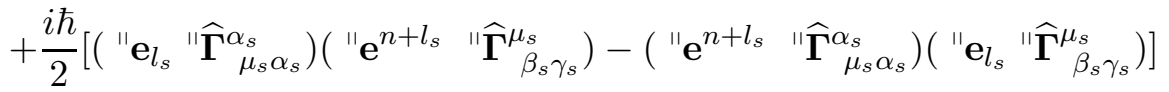

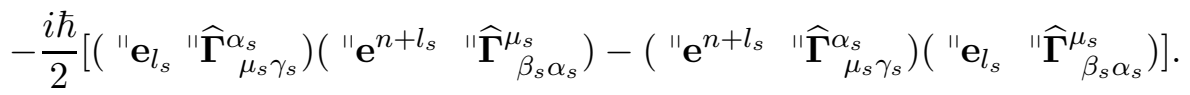

If we introduce in (A.28) the formulas for s-adapted coefficients of type ${ }_{[01]}^{\prime} \widehat{\Gamma}_{\star \alpha_{s} \beta_{s}}^{\nu_{s}}$ (A.24), we can complete the procedure of computation of such s-terms. We do not provide such cumbersome formulas, and possible explicit h1-v2-c3-c4 decompositions and similar ones with labels $[1,0],[1,1]$ etc., because they will be not used in this work.

Now, we show how to compute the $[1,0]$ terms of order $\kappa$. For ${ }_{[00]}^{\prime \prime} \widehat{\Gamma}_{\star \alpha_{s} \beta_{s}}^{\nu_{s}}=" \widehat{\Gamma}_{\alpha_{s} \beta_{s}}^{\nu_{s}}$ and ${ }_{[10]}^{\prime \prime} \widehat{\Gamma}_{\star \alpha_{s} \beta_{s}}^{\nu_{s}}$ from (A.23) and (A.24). Using, Kronecker s-adapted symbols of type $\delta_{\gamma_{s} k_{s}}$ and $\delta_{a_{s}}^{\gamma_{s}}$, we obtain such s-adapted components of the nonassociative Ricci s-tensor:

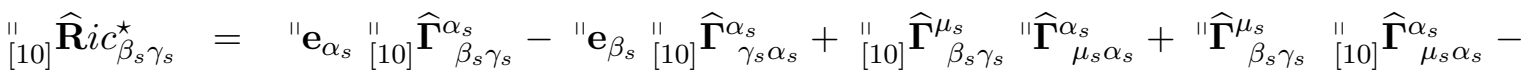

$$
\begin{aligned}
& { }_{[10]}^{\| 1} \widehat{\boldsymbol{\Gamma}}_{\beta_{s} \alpha_{s}}^{\mu_{s}} " \widehat{\boldsymbol{\Gamma}}_{\mu_{s} \gamma_{s}}^{\alpha_{s}}-" \widehat{\boldsymbol{\Gamma}}_{\beta_{s} \alpha_{s}[10]}^{\mu_{s}}{ }_{\mu_{s} \gamma_{s}}^{\alpha_{s}}+{ }^{\alpha_{s}} w_{\gamma_{s} \alpha_{s}}^{\mu_{s}}{ }_{\left.\mu_{s}\right]} \widehat{\boldsymbol{\Gamma}}_{\beta_{s} \mu_{s}}^{\alpha_{s}}+
\end{aligned}
$$

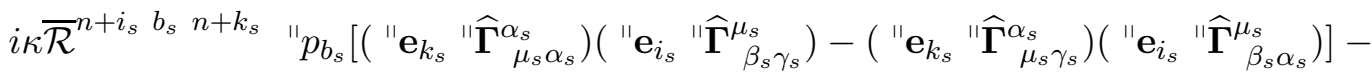

$$
\begin{aligned}
& i \kappa \overline{\mathcal{R}}^{n+i_{s} n+j_{s} n+k_{s}} \delta_{\gamma_{s} k_{s}} \mid \widehat{\boldsymbol{\Gamma}}_{\mu_{s} i_{s}}^{\alpha_{s}}\left({ }^{\prime \prime} \mathbf{e}_{j_{s}} " \widehat{\boldsymbol{\Gamma}}_{\beta_{s} \alpha_{s}}^{\mu_{s}}\right)+i \kappa \overline{\mathcal{R}}^{n+i_{s} n+j_{s} a_{s}} \delta_{a_{s}}^{\gamma_{s}}\left[" \mathbf{e}_{j_{s}}{ }^{\prime \prime} \mathbf{e}_{\gamma_{s}} \widehat{\boldsymbol{\Gamma}}_{\beta_{s} i_{s}}^{\alpha_{s}}-\right.
\end{aligned}
$$

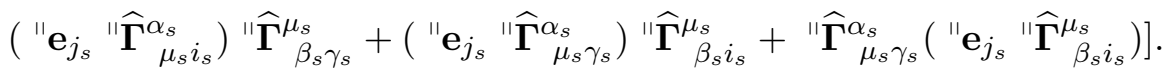

We note that the terms (A.28) and (A.29) for the star deformed Ricci s-tensor are imaginary as typical Nadapted configurations with commutative real N-connection structure studied in [3]. Such properties were found (in our case, for zero torsion distortions to LC-configurations) in 2]. We can consider also inverse transforms from holonomic configurations to N- and/ s-adapted ones. For instance, the coordinate formulas 
(5.78) - (5.79) from [2], for the LC-connection, are modified into respective s-formulas (A.28) and (A.29) for the canonical s-connection if we follow the Convention 2 (29) with such rules:

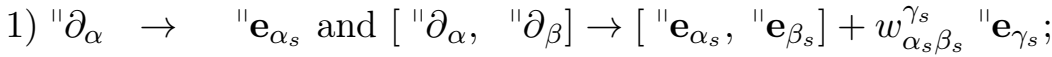

$$
\begin{aligned}
& \text { 2) " } \Gamma_{\beta \gamma}^{\mu} \rightarrow{ }^{\prime} \widehat{\boldsymbol{\Gamma}}_{\beta_{s} \gamma_{s}}^{\mu_{s}} \text {, i.e. " } \nabla \rightarrow{ }_{\mathbf{s}} \widehat{\mathbf{D}} \text {; and } " g_{\alpha \beta} \rightarrow{ }^{\prime \prime} \mathbf{g}_{\alpha_{s} \beta_{s}} \text {, etc. }
\end{aligned}
$$

In result, we can deform nonholonomically the formula (5.80) in [2] and compute the [11] $\hbar \kappa=\ell_{s}^{3} / 6$ contributions in dyadic form for the nonassociative canonical Ricci s-tensor (44),

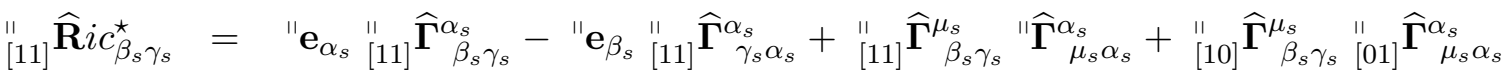

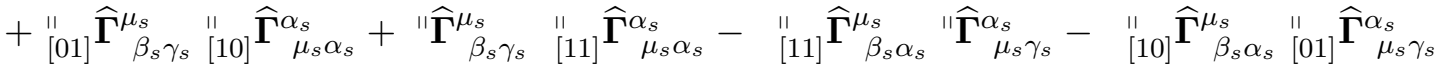

$$
\begin{aligned}
& -{ }_{[01]}^{\prime \prime} \widehat{\boldsymbol{\Gamma}}_{\beta_{s} \alpha_{s}[10]}^{\mu_{s}}{ }_{\mu_{s} \gamma_{s}}^{\prime \prime} \widehat{\boldsymbol{\Gamma}}^{\alpha_{s}}-" \widehat{\boldsymbol{\Gamma}}_{\beta_{s} \alpha_{s}[11]}^{\mu_{s}}{ }_{\mu_{s} \gamma_{s}}+\widehat{\boldsymbol{\Gamma}}^{\alpha_{s}}{ }_{\gamma_{s} \alpha_{s}[11]}^{\mu_{s}} \widehat{\boldsymbol{\Gamma}}_{\beta_{s} \mu_{s}}^{\alpha_{s}}
\end{aligned}
$$

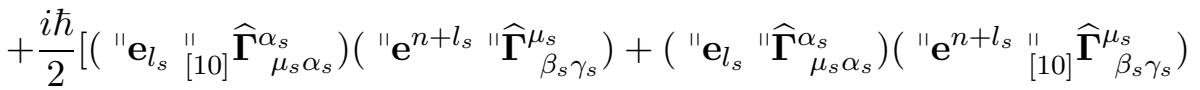

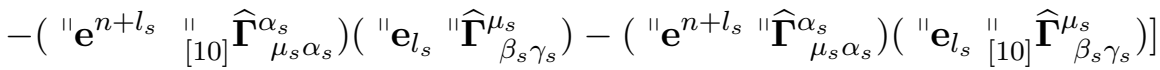

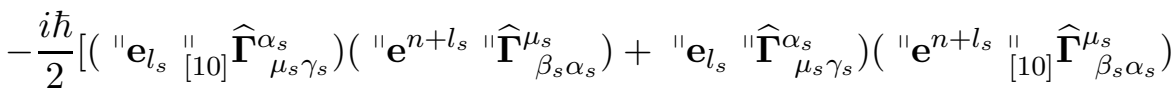

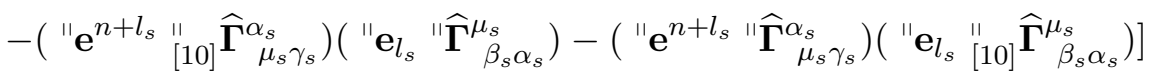

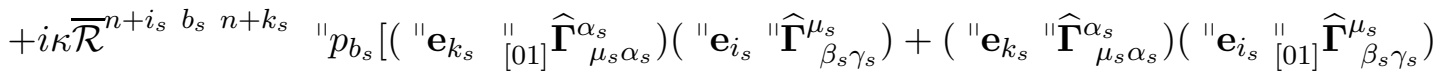

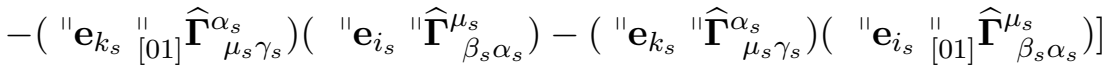

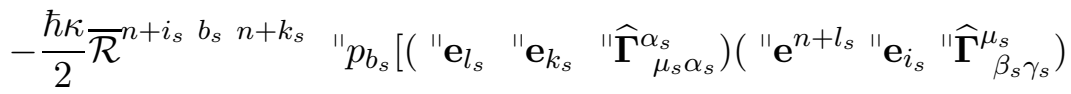

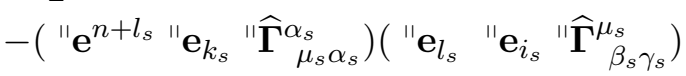

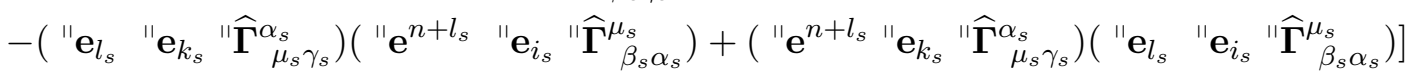

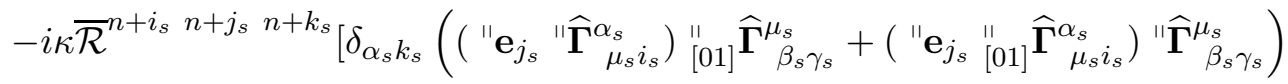

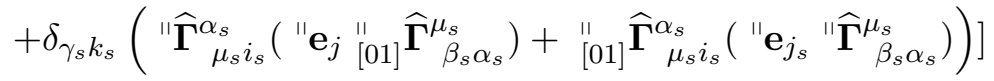

$$
\begin{aligned}
& +\frac{\hbar \kappa}{2} \overline{\mathcal{R}}^{n+i_{s} n+j_{s} n+k_{s}}\left[\delta_{\alpha_{s} k_{s}}\left(\left({ }^{\prime} \mathbf{e}_{l_{s}} " \mathbf{e}_{j_{s}} " \widehat{\boldsymbol{\Gamma}}_{\mu_{s} i_{s}}^{\alpha_{s}}\right)\left(" \mathbf{e}^{n+l_{s}} " \widehat{\boldsymbol{\Gamma}}_{\beta_{s} \gamma_{s}}^{\mu_{s}}\right)-\left(" \mathbf{e}^{n+l_{s}} " \mathbf{e}_{j_{s}} " \widehat{\boldsymbol{\Gamma}}_{\mu_{s} i_{s}}^{\alpha_{s}}\right)\left(" \mathbf{e}_{l_{s}} " \widehat{\boldsymbol{\Gamma}}_{\beta_{s} \gamma_{s}}^{\mu_{s}}\right)\right)\right.
\end{aligned}
$$

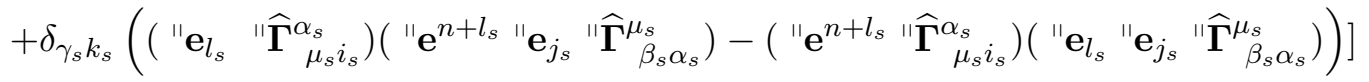

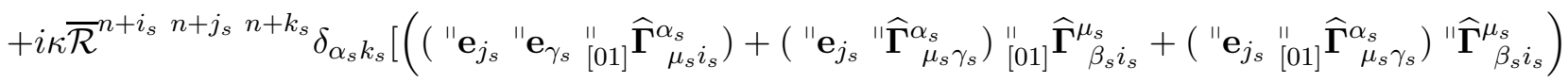

$$
\begin{aligned}
& \left.+" \widehat{\boldsymbol{\Gamma}}_{\mu_{s} \gamma_{s}}^{\alpha_{s}}\left(" \mathbf{e}_{j_{s}}{ }_{[01]}^{\prime \prime} \widehat{\boldsymbol{\Gamma}}_{\beta_{s} i_{s}}^{\mu_{s}}\right)+{ }_{[01]}^{\prime \prime} \widehat{\boldsymbol{\Gamma}}_{\mu_{s} \gamma_{s}}^{\alpha_{s}}\left({ }^{\prime \prime} \mathbf{e}_{j_{s}} " \widehat{\boldsymbol{\Gamma}}_{\beta_{s} i_{s}}^{\mu_{s}}\right)\right] \\
& -\frac{\hbar \kappa}{2} \overline{\mathcal{R}}^{n+i_{s} n+j_{s} n+k_{s}} \delta_{\alpha_{s} k_{s}}\left[\left("{ }^{\prime \prime} e_{l_{s}} " \mathbf{e}_{j_{s}} " \widehat{\boldsymbol{\Gamma}}_{\mu_{s} \gamma_{s}}^{\alpha_{s}}\right)\left(" \mathbf{e}^{n+l_{s}} " \widehat{\boldsymbol{\Gamma}}_{\beta_{s} i_{s}}^{\mu_{s}}\right)-\left(" \mathbf{e}^{n+l_{s}} " \mathbf{e}_{j_{s}} " \widehat{\boldsymbol{\Gamma}}_{\mu_{s} \gamma_{s}}^{\alpha_{s}}\right)\left(" \mathbf{e}_{l_{s}} " \widehat{\boldsymbol{\Gamma}}_{\beta_{s} i_{s}}^{\mu_{s}}\right)\right. \\
& \left.+\left(" \mathbf{e}_{l_{s}} " \widehat{\boldsymbol{\Gamma}}_{\mu_{s} \gamma_{s}}^{\alpha_{s}}\right)\left(" \mathbf{e}^{n+l_{s}} " \mathbf{e}_{j_{s}} " \widehat{\boldsymbol{\Gamma}}_{\beta_{s} i_{s}}^{\mu_{s}}\right)-\left(" \mathbf{e}^{n+l_{s}} " \widehat{\boldsymbol{\Gamma}}_{\mu_{s} \gamma_{s}}^{\alpha_{s}}\right)\left({ }^{\prime} \mathbf{e}_{l_{s}} " \mathbf{e}_{j_{s}} " \widehat{\boldsymbol{\Gamma}}_{\beta_{s} i_{s}}^{\mu_{s}}\right)\right] .
\end{aligned}
$$


To complete the computation of such real nonassociative and noncommutative contributions we have to insert formulas for coefficients of type ${ }_{[01]}^{\prime \prime} \widehat{\Gamma}_{\star \alpha \beta}^{\nu},{ }_{[10]}^{\prime \prime} \widehat{\Gamma}_{\star \alpha \beta}^{\nu}$ and ${ }_{[11]}^{\prime \prime} \widehat{\Gamma}_{\star \alpha \beta}^{\nu}$, see s-coefficients (A.23), (A.24) and (A.26). We omit cumbersome explicit formulas for [01],[10], and [11] because such nonassociative R-flux contributions can be encoded into an effective source for nonholonomic canonically deformed Einstein equations. Using effective sources with respective parameters and associative and commutative [00] canonical Ricci s-tensors, the solutions of physically important systems of nonlinear PDEs can be computed using the AFCDM outlined in $[20,21]$.

Finally, we note that nonassociative LC-connections can be extracted for nonholonomic constraints resulting in ${ }_{s} \widehat{\mathbf{D}}_{|s|}^{\star} \widehat{\mathbf{T}=0}=" \nabla^{\star}$, see (42). In coordinate bases, we obtain the formulas for parametric Ricci tensor (5.80) from [2]. It is important to have a prescription with Rules 1-2 (A.30) in order to move geometric s-objects from local coordinate bases to s-adapted bases, and inversely, determined by canonical data $\left(" \mathbf{e}_{\alpha_{s}}, " \mathbf{g}_{\alpha_{s} \beta_{s}}, " \widehat{\boldsymbol{\Gamma}}_{\beta_{s} \gamma_{s}}^{\mu_{s}}\right)$.

\section{B Nonholonomic dyadic deformations into parametric solutions}

In this appendix, we reformulate for nonassociative gravity models the results on section 5 from [40]. Such results are important for constructing exact and parametric locally anisotropic $\mathrm{BH}$ and cosmological solutions generated by nonassociative star nonholonomic deformations and related effective R-flux sources.

\section{B.1 Using s-metric coefficients as generating functions}

Using nonlinear symmetries (98), we obtain formulas:

$$
\begin{aligned}
& \text { shell } s=2: \quad\left[\left({ }_{2} \Psi\right)^{2}\right]^{\diamond}=-\int d y^{3}\left({ }_{2}{ }_{2} \mathcal{K}\right) g_{4}^{\diamond}, \quad\left({ }_{2} \Phi\right)^{2}=-4{ }_{2} \Lambda_{0} g_{4} ; \\
& \text { shell } s=3: \quad \text { " } \partial^{6}\left[\left({ }_{3}^{\prime \prime} \Psi\right)^{2}\right]=-\int d{ }^{\prime \prime} p_{6}\left({ }_{3}^{\prime \prime} \mathcal{K}\right) \quad " \partial^{6} " g^{5}, \quad\left({ }_{3}^{\prime \prime} \Phi\right)^{2}=-4{ }_{3}^{\|} \Lambda_{0} " g^{6} ; \\
& \text { shell } s=4: \quad\left[\left({ }_{4}^{\prime \prime} \Psi\right)^{2}\right]^{*}=-\int d{ }^{"} E\left({ }_{4}^{\prime \prime} \mathcal{K}\right)\left({ }^{\prime \prime} g^{7}\right)^{*}, \quad\left({ }_{4}^{\prime \prime} \Phi\right)^{2}=-4{ }_{4}^{" ~} \Lambda " g^{8} .
\end{aligned}
$$

We conclude that the quadratic elements for quasi-periodic solutions (89) and/or (99) can be rewritten equivalently in terms of generating data $\left(g_{4}, " g^{5}, " g^{7} ;{ }_{s} \Lambda\right)$ and respective nonlinear symmetries involving generating sources ${ }_{s}^{\|} \mathcal{K}$,

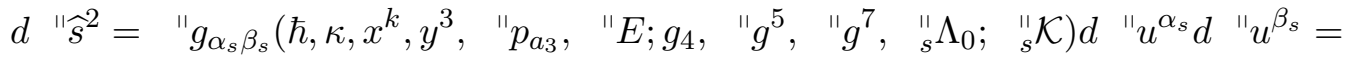

$$
\begin{aligned}
& e^{\psi\left(\hbar, \kappa, x^{k_{1}}\right)}\left[\left(d x^{1}\right)^{2}+\left(d x^{2}\right)^{2}\right]-\frac{\left(g_{4}^{\diamond}\right)^{2}}{\left|\int d y^{3}\left[\left({ }_{2}^{\prime \prime} \mathcal{K}\right) g_{4}\right]^{\diamond}\right| g_{4}}\left\{d y^{3}+\frac{\partial_{i_{1}}\left[\int d y^{3}\left({ }_{2}^{\prime \prime} \mathcal{K}\right) g_{4}^{\diamond}\right]}{(\underset{2}{\|} \mathcal{K}) g_{4}^{\diamond}} d x^{i_{1}}\right\}^{2}+ \\
& g_{4}\left\{d t+\left[{ }_{1} n_{k_{1}}+{ }_{2} n_{k_{1}} \int d y^{3} \frac{\left(g_{4}^{\diamond}\right)^{2}}{\left|\int d y^{3}\left[\left({ }_{2} \mathcal{K}\right) g_{4}\right]^{\diamond}\right|\left[g_{4}\right]^{5 / 2}}\right] d x^{k_{1}}\right\}+ \\
& " g^{5}\left\{d " p_{5}+\left[{ }_{1}^{11} n_{k_{2}}+{ }_{2}{ }_{2} n_{k_{2}} \int d " p_{6} \frac{\left[" \partial^{6}\left(" g^{5}\right)\right]^{2}}{\left|\int d " p_{6}{ }^{"} \partial^{6}\left[\left({ }_{3}^{\prime \prime} \mathcal{K}\right) " g^{5}\right]\right|\left[" g^{5}\right]^{5 / 2}}\right] d x^{k_{2}}\right\}-
\end{aligned}
$$

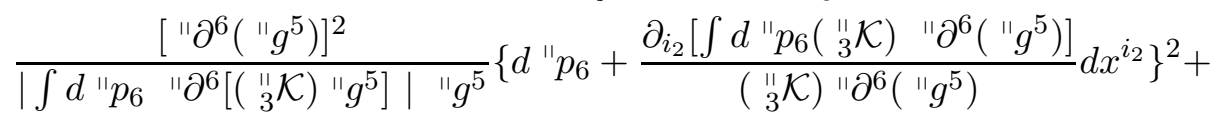

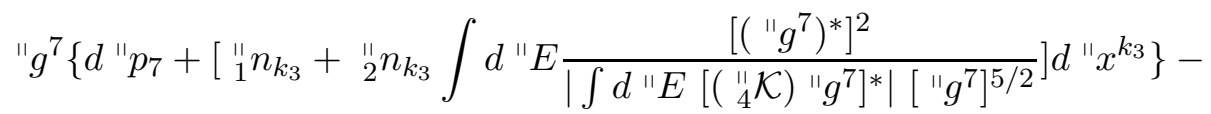

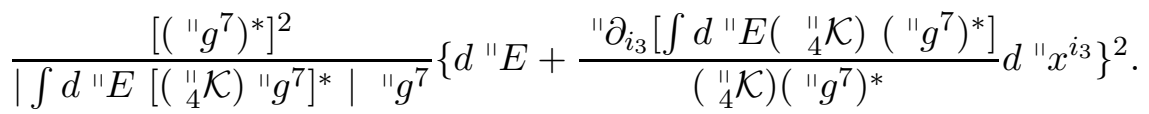


The parametric dependence, signs and integration functions/constants in above formulas should to be chosen some forms which are compatible with experimental/ observational data or corrections from R-fluxes in string theories.

In a similar form we can generate LC-configurations using data

$$
\check{g}_{4}\left(\hbar, \kappa, x^{i_{1}}, y^{3}\right), " \check{g}^{5}\left(\hbar, \kappa, x^{i_{1}}, x^{a_{2}}, " \text { " } p_{6}\right), " \check{g}^{7}\left(\hbar, \kappa, x^{i_{1}}, x^{a_{2}}, " p_{a_{2}}, " \textrm{ } E\right)
$$

and nonlinear symmetries (94) for quasi-stationary solutions (95). We omit such formulas (for associative and commutative configurations, similar results are stated pe Remark 5.1 and formula (64) in [40]). The R-flux contributions of effective sources ${ }_{s} \mathcal{K}$ are encoded correspondingly in N-connection coefficients ${ }_{s} \check{A}$ from (95).

\section{B.2 Polarization parametric functions for nonassociative prime and target s-metrics}

On a phase space (for simplicity, we can consider an associative/commutative variant modelled by a dual Lorentz bundle enabled with nonholonomic dyadic structure, "e ${ }^{\alpha_{s}} \in T_{s}^{*} \mathbf{T}_{\|}^{*} \mathbf{V}$ (8), we consider a prime smetric ${ }_{s} \mathrm{~g}$ structure (15), with possible star deformations to respective symmetric and nonsymmetric s-metrics of type (16) and (17). In s-adapted form, we write

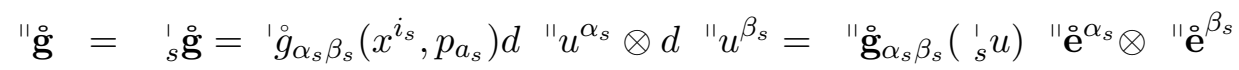

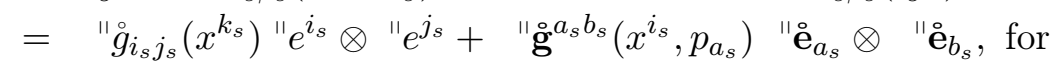

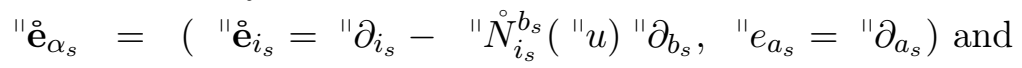

$$
\begin{aligned}
& " \mathrm{e}^{\alpha_{s}}=\left(d " x^{i_{s}}, " \mathrm{e}^{a_{s}}=d " y^{a_{s}}+"{ }^{N_{i_{s}}^{a_{s}}}(" u) d " x^{i_{s}}\right) \text {. }
\end{aligned}
$$

We label prime s-metrics and related geometric s-objects with a small circle on the left/right/up of corresponding symbols. Such a prime ${ }_{s}^{\prime \prime} \mathrm{g}(\overline{\mathrm{B} .2})$ my be, or not, a solution of certain gravitational field equations in a MGT or GR with phase space extension, which may result in parametric deformations to nonassociative con-

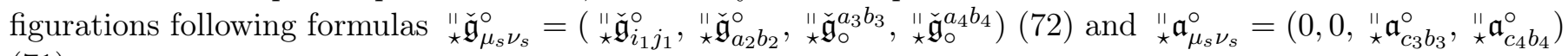
(171).

For nontrivial R-flux and star deformations, we can study nonassociative parametric nonholonomic deformations to nonlinear quadratic elements determined by target quasi-stationary s-metrics of type ${ }_{s} \mathbf{g}$ (89) as solutions of nonassociative vacuum Einstein equations (70). Such nonholonomic star s-deformations can be described in terms of so-called gravitational polarization ( $\eta$-polarization) functions,

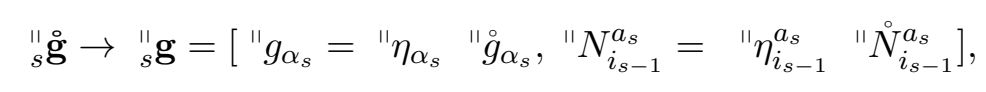

when the target s-metrics are written

$$
\begin{aligned}
& { }_{s} \mathbf{g}=" g_{i_{s}}\left(\hbar, \kappa, " x^{k_{s}}\right) d " x^{i_{s}} \otimes d " x^{i_{s}}+" g_{a_{s}}\left(\hbar, \kappa, " x^{i_{s}}, " p_{b_{s}}\right) \quad " \mathrm{e}^{a_{s}} \otimes " \mathrm{e}^{a_{s}} \\
& =\quad " \eta_{i_{k}}\left(\hbar, \kappa, x^{i_{1}}, y^{a_{2}}, " p_{a_{3}}, " p_{a_{4}}\right) \quad "{\stackrel{\circ}{i_{s}}}\left(\hbar, \kappa, x^{i_{1}}, y^{a_{2}}, " p_{a_{3}}, " p_{a_{4}}\right) d " x^{i_{s}} \otimes d " x^{i_{s}}
\end{aligned}
$$

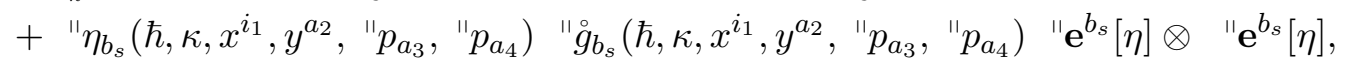

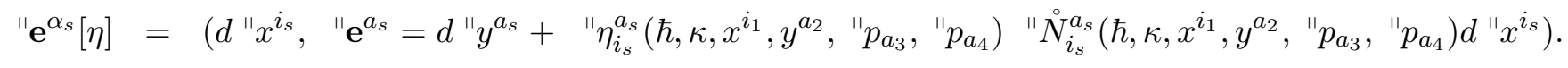

We emphasize that any of multiples in a " $\eta$ " $g$ may depend, in principle, on mixed shell coordinates and various parameters, sources, etc. To apply the AFCDM we have to use products subjected to the condition that the target s-metrics (with the coefficients in the left sides) are adapted to ordered shell coordinates. We use the term "gravitational polarizations" because for $\eta$-deformations with a small parameter, we can generate solutions, for instance, of black hole/ ellipsoid type but with effective polarization of fundamental physical constants (for (non) commutative MGTs, see solutions from [17, 44, 18, 41, 4, 20, 5, 21, 40] and reference therein). 
For any prescribed prime s-metric ${ }_{s}^{\|} \mathrm{g}$, we can consider as generating functions some data given by $\eta$ polarizations, for instance, by " $\eta_{4}\left(\hbar, \kappa, x^{i_{1}}, y^{3}\right)$,

" $\eta^{5}\left(\hbar, \kappa, x^{i_{1}}, y^{a_{1}}, " p_{6}\right)$, " $\eta^{7}\left(\hbar, \kappa, x^{i_{1}}, y^{a_{1}}, " p_{a_{2}}\right.$, "E) which should be defined from the condition that the target s-metric is a quasi-stationary solution equivalent to ${ }_{s}^{1} \mathbf{g}(89)$. By straightforward computations, we can check such solutions parameterized as (B.3) ad described by nonlinear quadratic elements with explicit dependence on $\eta$-polarizations,

$$
\begin{aligned}
& d^{\prime \prime} \widehat{s}^{2}=" g_{\alpha_{s} \beta_{s}}\left(\hbar, \kappa, x^{k}, y^{3}, " p_{a_{3}}, " p_{a_{4}} ; g_{4}, " g^{5}, " g^{7},{ }_{s}{ }_{s} \Lambda_{0} ;{ }_{s}{ }_{s} \mathcal{K}\right) d "{ }^{\prime \prime} u^{\alpha_{s}} d " u^{\beta_{s}} \\
& =e^{\psi\left(\hbar, \kappa, x^{k_{1}}\right)}\left[\left(d x^{1}\right)^{2}+\left(d x^{2}\right)^{2}\right]-\frac{\left.\left[\left(" \eta_{4} "{ }^{\circ}\right)_{4}\right)^{\diamond}\right]^{2}}{\left.\mid \int d y^{3}\left({ }_{2}^{\prime \prime} \mathcal{K}\right)\left(" \eta_{4} "{ }^{\circ}\right)_{4}\right)^{\diamond} \mid\left(" \eta_{4} "{ }^{\circ} g_{4}\right)}\left\{d y^{3}+\frac{\partial_{i_{1}}\left[\int d y^{3}\left({ }_{2} \mathcal{K}\right)\left(" \eta_{4} "{ }^{\circ} g_{4}\right)^{\diamond}\right]}{\left({ }_{2} \mathcal{K}\right)\left(" \eta_{4} "{ }^{\circ} g_{4}\right)^{\diamond}} d x^{i_{1}}\right\}^{2}
\end{aligned}
$$

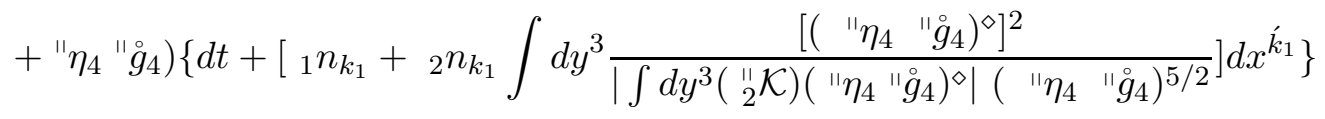

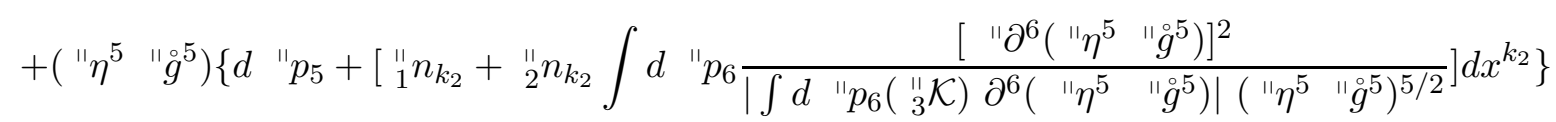

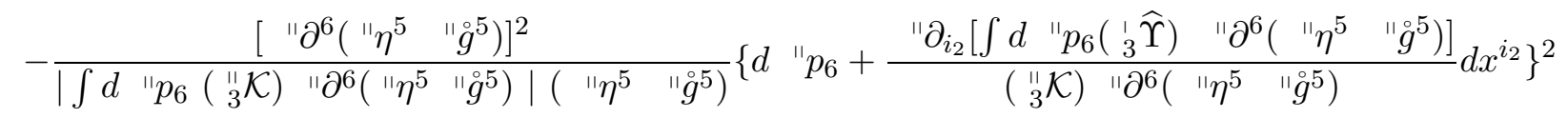

$$
\begin{aligned}
& +\quad\left(" \eta^{7} "{ }^{7}\right)\left\{d " p_{7}+\left[{ }_{1} n_{k_{3}}+{ }_{2} n_{k_{3}} \int d " E \frac{\left[\left(" \eta^{7} " \stackrel{g}{g}^{7}\right)^{*}\right]^{2}}{\left|\int d " E\left({ }_{4} \mathcal{K}\right)\left[\left(" \eta^{7} " g^{7}\right)\right]^{*}\right|\left[\left(" \eta^{7} "{ }^{\circ} g^{7}\right)\right]^{5 / 2}}\right] d " x^{k_{3}}\right\}
\end{aligned}
$$

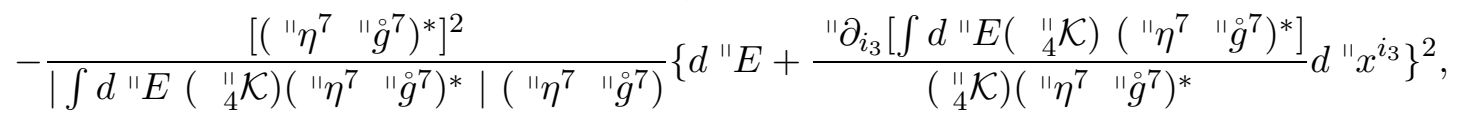


in quasi-stationary solutions of type (89) and/or (99):

$$
\begin{aligned}
& \text { "g } g_{i_{1}}\left(\kappa, x^{k_{1}}\right)=" \eta_{i_{i}} \quad{ }^{\circ}{\stackrel{\circ}{i_{1}}}=" \zeta_{i_{1}}\left(1+\kappa " \chi_{i_{1}}\right) \quad "{\stackrel{\circ}{g_{1}}}=
\end{aligned}
$$

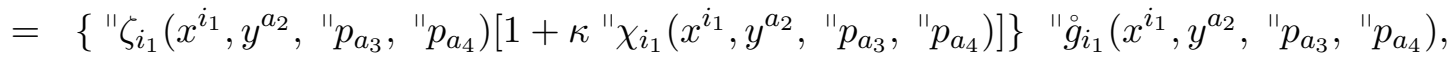

$$
\begin{aligned}
& " g_{b_{2}}\left(\kappa, x^{i_{1}}, y^{3}\right)=\quad " \eta_{b_{2}} \quad " \stackrel{\circ}{g_{b_{1}}}=" \zeta_{b_{2}}\left(1+\kappa " \chi_{b_{2}}\right) \quad " \stackrel{\circ}{g}_{b_{1}}= \\
& =\left\{" \zeta_{b_{2}}\left(x^{i_{1}}, y^{a_{2}}, " p_{a_{3}}, " p_{a_{4}}\right)\left[1+\kappa " \text { " } \chi_{b_{2}}\left(x^{i_{1}}, y^{a_{2}}, " p_{a_{3}}, " p_{a_{4}}\right)\right]\right\} \quad \text { " }{ }_{b_{1}}\left(x^{i_{1}}, y^{a_{2}}, " p_{a_{3}}, " p_{a_{4}}\right), \\
& " g^{a_{3}}\left(\kappa, x^{i_{2}}, " p_{6}\right)=" \eta^{a_{3}} "{ }^{a^{a_{3}}}=" \zeta^{a_{3}}\left(1+\kappa " \chi^{a_{3}}\right) " \stackrel{\circ}{g} a^{a_{3}}= \\
& =\left\{{ }^{\prime a^{a_{3}}}\left(x^{i_{1}}, y^{b_{2}}, " p_{b_{3}}, " p_{b_{4}}\right)\left[1+\kappa{ }^{\prime \prime} \chi^{a_{3}}\left(x^{i_{1}}, y^{b_{2}}, " p_{b_{3}}, " p_{b_{4}}\right)\right]\right\}{ }^{\prime \circ}{ }^{a_{3}}\left(x^{i_{1}}, y^{b_{2}}, " p_{b_{3}}, " p_{b_{4}}\right) \text {, }
\end{aligned}
$$

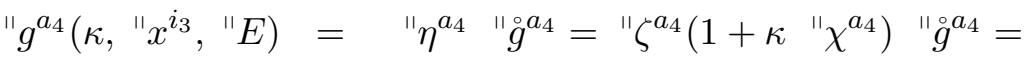

$$
\begin{aligned}
& =\left\{" \zeta^{a_{4}}\left(x^{i_{1}}, y^{b_{2}}, " p_{b_{3}}, " p_{b_{4}}\right)\left[1+\kappa " \chi^{a_{4}}\left(x^{i_{1}}, y^{b_{2}}, " p_{b_{3}}, " p_{b_{4}}\right)\right]\right\} \quad " \stackrel{a^{a_{4}}}{ }\left(x^{i_{1}}, y^{a_{2}}, " p_{a_{3}}, " p_{a_{4}}\right) \text {. }
\end{aligned}
$$

Similar $\kappa$-decompositions can be used for N-connection s-coefficients

$$
\begin{aligned}
& \text { " } N_{i_{1}}^{a_{2}}\left(\kappa, x^{k_{1}}, y^{3}\right)=" \eta_{i_{1}}^{a_{2}} \quad "{\stackrel{\circ}{i_{1}}}_{i_{2}}^{a_{2}}=" \zeta_{i_{1}}^{a_{2}}\left(1+\kappa " \chi_{i_{1}}^{a_{2}}\right) \quad "{\stackrel{\circ}{i_{1}}}^{a_{2}}=
\end{aligned}
$$

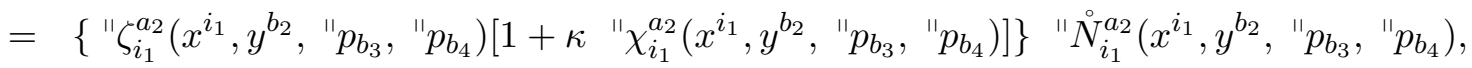

$$
\begin{aligned}
& \text { " } N_{i_{2} a_{3}}\left(\kappa, x^{k_{1}}, y^{b_{2}}, " p_{6}\right)=" \eta_{i_{2} a_{3}} \quad "{\stackrel{\circ}{N} i_{2} a_{3}}=" \zeta_{i_{2} a_{3}}\left(1+\kappa " \chi_{i_{2} a_{3}}\right) "{ }^{\prime}{\stackrel{\circ}{i_{2} a_{3}}}= \\
& =\left\{\text { " } \zeta_{i_{2} a_{3}}\left(x^{i_{1}}, y^{b_{2}}, " p_{b_{3}}, " p_{b_{4}}\right)\left[1+\kappa "{ }^{\prime \prime} \chi_{i_{2} a_{3}}\left(x^{i_{1}}, y^{b_{2}}, " p_{b_{3}}, " p_{b_{4}}\right)\right]\right\}{ }^{\prime}{\stackrel{\circ}{i_{2} a_{3}}}\left(x^{i_{1}}, y^{b_{2}}, " p_{b_{3}}, " p_{b_{4}}\right) \text {, } \\
& \text { " } N_{i_{3} a_{4}}\left(\kappa, x^{k_{1}}, y^{b_{2}}, " p_{a_{3}}, " E\right)=" \eta_{i_{3} a_{4}} \quad "{\stackrel{\circ}{i_{3} a_{4}}}=" \zeta_{i_{3} a_{4}}\left(1+\kappa " \chi_{i_{3} a_{4}}\right) \quad " N_{i_{3} a_{4}}= \\
& =\left\{" \zeta_{i_{3} a_{4}}\left(x^{i_{1}}, y^{b_{2}}, " p_{b_{3}}, " p_{b_{4}}\right)\left[1+\kappa \chi_{i_{3} a_{4}}\left(x^{i_{1}}, y^{b_{2}}, " p_{b_{3}}, " p_{b_{4}}\right)\right]\right\} \quad "{\stackrel{\circ}{i_{3} a_{4}}}\left(x^{i_{1}}, y^{b_{2}}, " p_{b_{3}}, " p_{b_{4}}\right) \text {. }
\end{aligned}
$$

In brief, we write above formulas on star parametric deformations of a s-metric and N-connection structure on ${ }_{s} \mathbf{T}_{\|}^{*} \mathbf{V}$ in the form

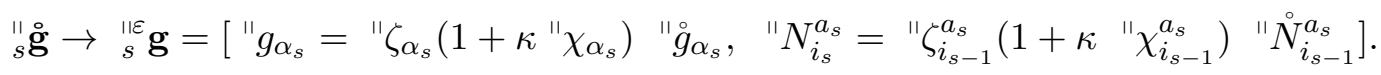

The $\zeta$ - and $\chi$-coefficients for deformations (B.5) are generated by shell data

$$
" \eta_{2}=" \zeta_{2}\left(1+\kappa " \chi_{2}\right), \quad " \eta_{4}=" \zeta_{4}\left(1+\kappa " \chi_{4}\right), \quad " \eta^{5}=" \zeta^{5}\left(1+\kappa " \chi^{5}\right), \quad " \eta^{7}={ }^{" \prime} \zeta^{7}\left(1+\kappa " \chi^{7}\right) \text {. }
$$

Let us explain below how such parametric deformations are performed on respective shells:

For $s=1$, we can consider " $\zeta_{i_{1}}=\left({ }^{\prime \prime}{\stackrel{\circ}{i_{1}}}\right)^{-1} e^{\psi_{0}\left(x^{k_{1}}\right)}$ and " $\chi_{i_{1}}=\left("{\stackrel{\circ}{i_{1}}}\right)^{-1} \psi$ " $\chi\left(x^{k_{1}}\right)$, where

$$
\text { " } \zeta_{i_{1}}\left(1+\kappa^{\prime \prime} \chi_{i_{1}}\right) \quad "{\stackrel{\circ}{g_{1}}}_{i_{1}}=e^{\psi\left(x^{k_{1}}\right)} \approx e^{\psi_{0}\left(x^{k_{1}}\right)\left(1+\kappa^{\psi} \chi\left(x^{k_{1}}\right)\right)} \approx e^{\psi_{0}\left(x^{k_{1}}\right)}\left(1+\kappa^{\psi}{ }^{\|} \chi\right)
$$

for $\psi_{0}\left(x^{k_{1}}\right)$ and " $\chi\left(x^{k_{1}}\right)$ defined by a solution of a 2-d Poisson equation (88).

Then, for $s=2$ (with generating functions " $\zeta_{4}$ and " $\chi_{4}$; generating source and cosmological constant, " $\mathcal{2}$,

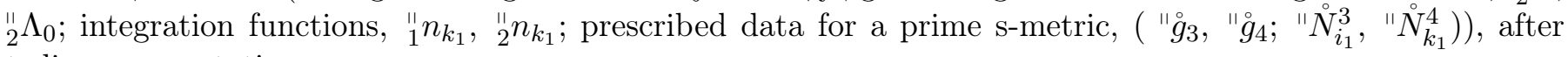
tedious computations, we express

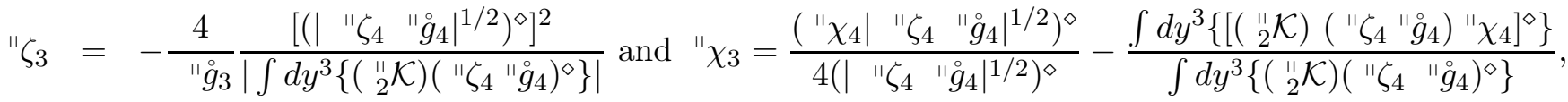

$$
\begin{aligned}
& " \zeta_{i_{1}}^{3}=\frac{\partial_{i_{1}} \int d y^{3}\left({ }_{2}{ }_{2} \mathcal{K}\right)\left(" \zeta_{4}\right)^{\diamond}}{\left(" N_{i_{1}}^{3}\right)\left({ }_{2}{ }_{2} \mathcal{K}\right)\left(" \zeta_{4}\right)^{\diamond}} \text { and " } \chi_{i_{1}}^{3}=\frac{\partial_{i_{1}}\left[\int d y^{3}\left({ }_{2}{ }_{2} \mathcal{K}\right)\left(" \zeta_{4} " \chi_{4}\right)^{\diamond}\right]}{\partial_{i_{1}}\left[\int d y^{3}\left({ }_{2}{ }_{2} \mathcal{K}\right)\left(" \zeta_{4}\right)^{\diamond}\right]}-\frac{\left(" \zeta_{4} " \chi_{4}\right)^{\diamond}}{\left(" \zeta_{4}\right)^{\diamond}},
\end{aligned}
$$

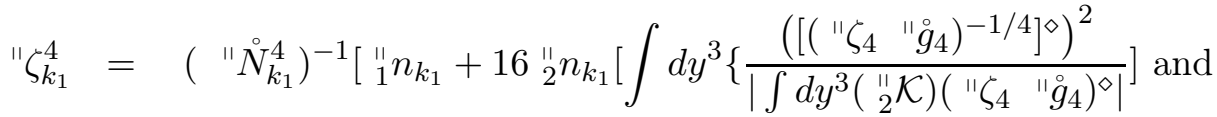

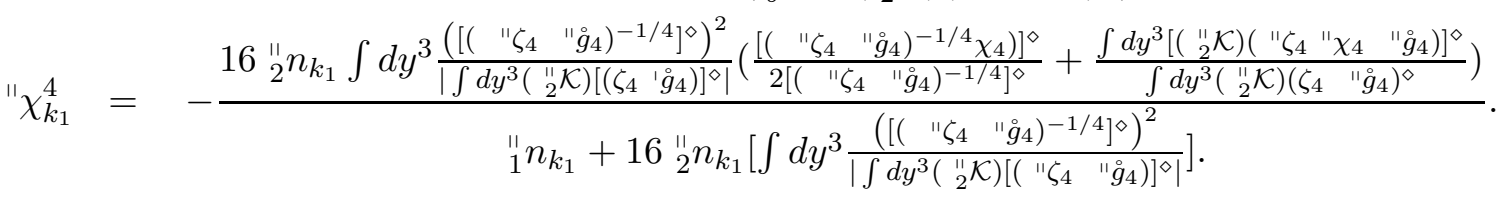


Such formulas can be obtained for $s=3$ (with generating functions, " $\zeta^{5}$, " $\chi^{5}$; generating source and cosmological constant , ${ }_{3}^{11} \mathcal{K},{ }_{3}^{11} \Lambda_{0}$; integration functions, ${ }_{1}^{11} n_{k_{3}},{ }_{2}^{11} n_{k_{3}}$; prescribed data for a prime s-metric, $\left(" \stackrel{\circ}{g}^{5}, " \stackrel{\circ}{g}^{6} ; " \stackrel{\circ}{N_{k_{2}}}, " \stackrel{\circ}{N}_{i_{2} 6}\right)$ :

$$
\begin{aligned}
& " \zeta^{6}=-\frac{4}{" g^{6}} \frac{\left[" \partial^{6}\left(\left|" \zeta^{5} \quad " g^{5}\right|^{1 / 2}\right)\right]^{2}}{\left|\int d " p_{6}\left\{\left(\begin{array}{cc}
11 \\
3
\end{array}\right){ }^{\prime \prime} \partial^{6}\left(" \zeta^{5} \quad " g^{5}\right)\right\}\right|} \text { and }
\end{aligned}
$$

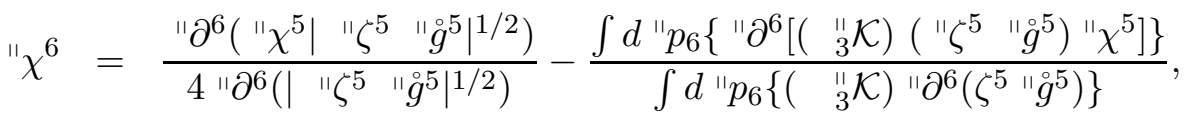

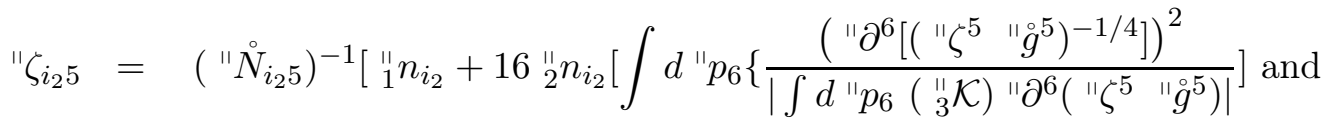

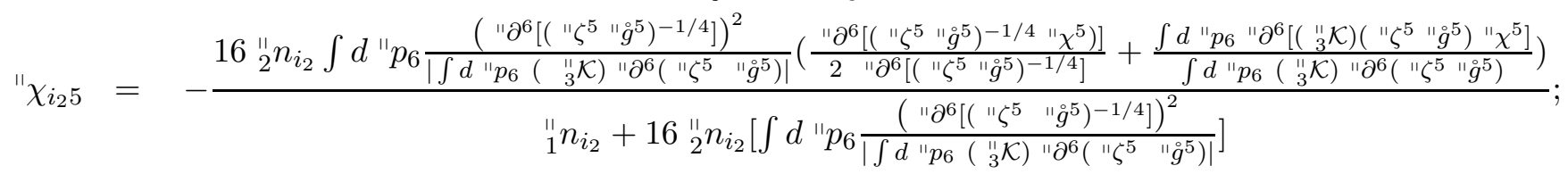

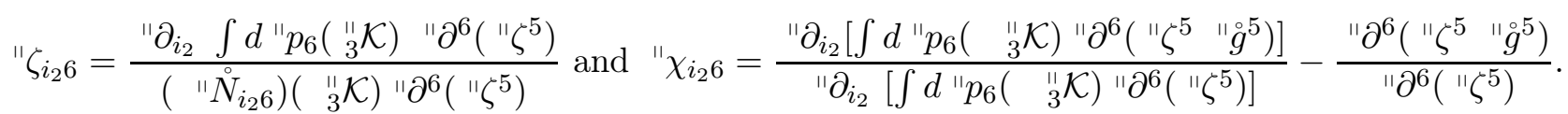

Finally, we provide the formulas for the shell $s=4$ (with generating functions, " $\zeta^{7}$, " $\chi^{7}$; generating source and cosmological constant, ${ }_{4}^{11} \mathcal{K},{ }_{4}^{11} \Lambda_{0}$; integration functions, ${ }_{1}^{11} n_{k_{4}},{ }_{2}^{11} n_{k_{4}}$; prescribed data for a prime s-metric, $\left(" \stackrel{\circ}{g}^{7}, " \stackrel{\circ}{g}^{8} ; " N_{k_{3} 7}, " \stackrel{\circ}{i_{3} 8}\right)$ :

$$
\begin{aligned}
& " \zeta^{8}=-\frac{4}{" g^{8}} \frac{\left[\left(\left|" \zeta^{7} " g^{7}\right|^{1 / 2}\right)^{*}\right]^{2}}{\left|\int d " E\left\{\left("{ }_{4} \mathcal{K}\right)\left[\left(" \zeta^{7} " g^{7}\right)\right]^{*}\right\}\right|} \text { and } \\
& " \chi^{8}=\frac{\left(" \chi^{7}\left|" \zeta^{7} " g^{7}\right|^{1 / 2}\right)^{*}}{4\left(\left.\left|" \zeta^{7} "{ }^{\circ}\right|^{7 / 2}\right|^{1 / 2}\right.}-\frac{\left.\int d " E\left\{\left[\left({ }_{4}^{\prime \prime} \mathcal{K}\right)\left(" \zeta^{7} "{ }^{7}\right)^{n}\right)^{n} \chi^{7}\right]^{*}\right\}}{\int d " E\left\{\left({ }_{4} \mathcal{K}\right)\left(" \zeta^{4} "{ }^{\circ} g^{4}\right)^{*}\right\}},
\end{aligned}
$$

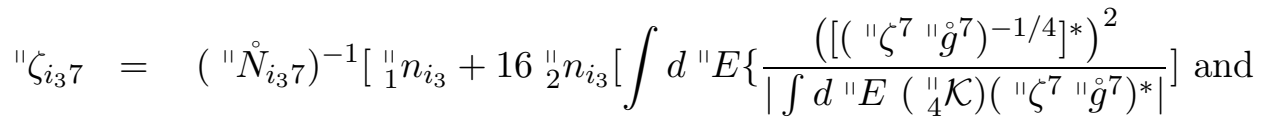

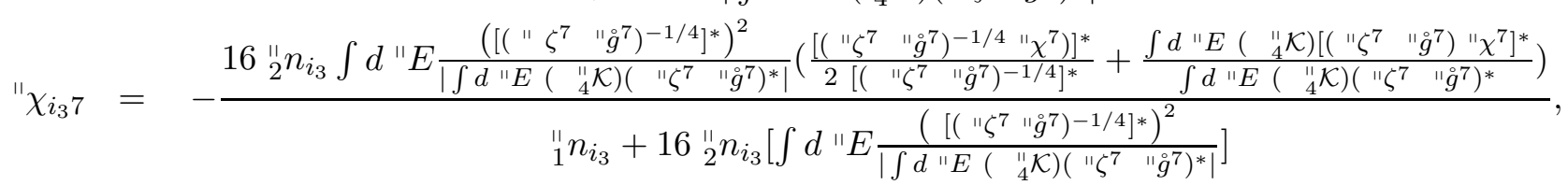

$$
\begin{aligned}
& " \zeta_{i_{3} 8}=\frac{\left." \partial_{i_{3}} \int d{ }^{" ~} E\left({ }_{4}\right) \mathcal{K}\right)\left(" \zeta^{7}\right)^{*}}{\left("{ }_{i_{3} 8}\right)\left({ }_{4} \mathcal{K}\right)\left(" \zeta^{7}\right)^{*}} \text { and }{ }^{\prime \prime} \chi_{i_{3} 7}=\frac{\left." \partial_{i_{3}}\left[\int d " E\left({ }_{4} \mathcal{K}\right)\left(" \zeta^{7} "{ }^{\circ}\right)^{7}\right)^{*}\right]}{" \partial_{i_{3}}\left[\int d " E\left({ }_{4} \mathcal{K}\right)\left(" \zeta^{7}\right)^{*}\right]}-\frac{\left(" \zeta^{7} " g^{7}\right)^{*}}{\left(" \zeta^{7}\right)^{*}} \text {. }
\end{aligned}
$$

Similar formulas for associative and commutative phase spaces are stated by Theorem 5.1 and proven in appendix A.6 of [40] in terms of different type dyadic variables and effective sources.

Introducing above $\kappa$-coefficients instead of $\eta$-coefficients of (B.4), we obtain such nonlinear quadratic elements for quasi-stationary solutions,

$$
\begin{aligned}
& d " \widehat{s}^{2}=" \widehat{g}_{\alpha_{s} \beta_{s}}\left(x^{k}, y^{3}, " p_{a_{3}}, " p_{a_{4}} ; g_{4}, " g^{5}, " g^{7},{ }_{s}{ }_{s} \mathcal{K},{ }_{s} \Lambda_{0}, \kappa\right) d " u^{\alpha_{s}} d^{" \prime} u^{\beta_{s}}=e^{\psi_{0}\left(x^{k_{1}}\right)}(1+\kappa \psi " \chi)\left[\left(d x^{1}\right)^{2}+\left(d x^{2}\right)^{2}\right]
\end{aligned}
$$

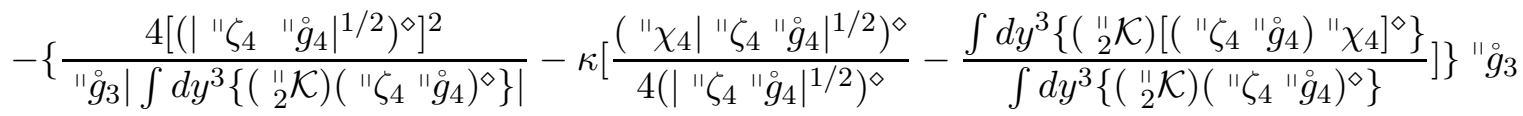

$$
\begin{aligned}
& +\left\{d y^{3}+\left[\frac{\partial_{i_{1}} \int d y^{3}\left({ }_{2}^{\prime \prime} \mathcal{K}\right)\left(" \zeta_{4}\right)^{\diamond}}{\left(" N_{i_{1}}^{3}\right)\left({ }_{2}^{\prime \prime} \mathcal{K}\right)\left(" \zeta_{4}\right)^{\diamond}}+\kappa\left(\frac{\partial_{i_{1}}\left[\int d y^{3}\left({ }_{2}^{\prime \prime} \mathcal{K}\right)\left(" \zeta_{4} " \chi_{4}\right)^{\diamond}\right]}{\partial_{i_{1}}\left[\int d y^{3}\left({ }_{2}{ }_{2} \mathcal{K}\right)\left(" \zeta_{4}\right)^{\diamond}\right]}-\frac{\left(" \zeta_{4} " \chi_{4}\right)^{\diamond}}{\left(" \zeta_{4}\right)^{\diamond}}\right)\right]\left(" \stackrel{\circ}{N}_{i_{1}}^{3}\right) d x^{i_{1}}\right\}^{2}
\end{aligned}
$$




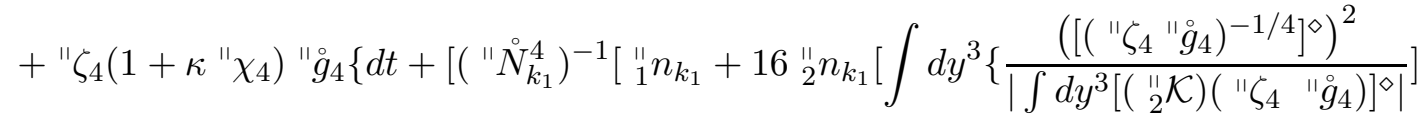

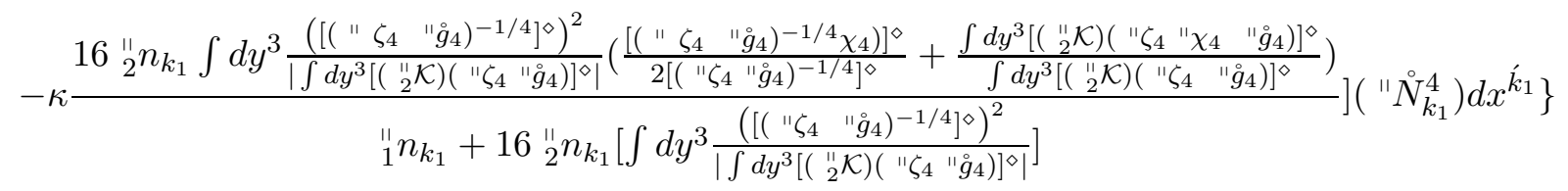

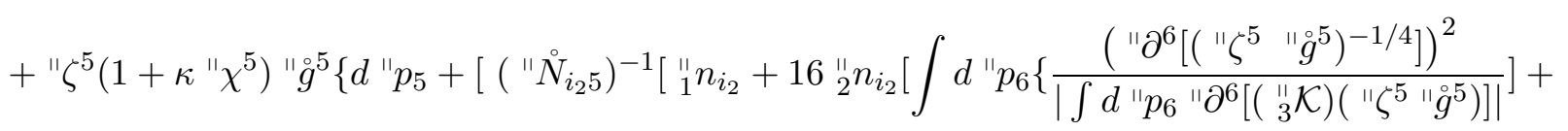

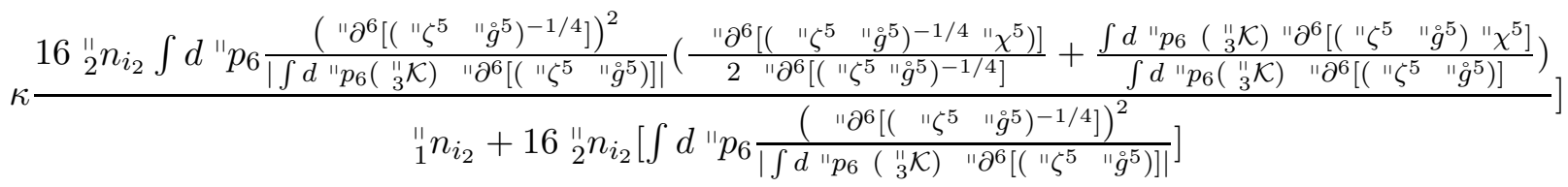

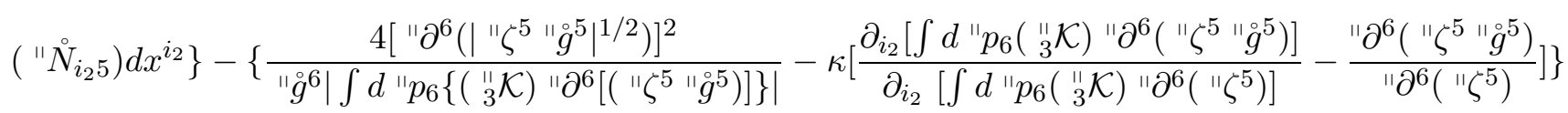

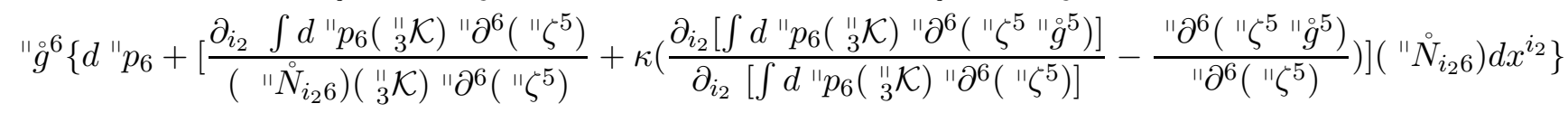

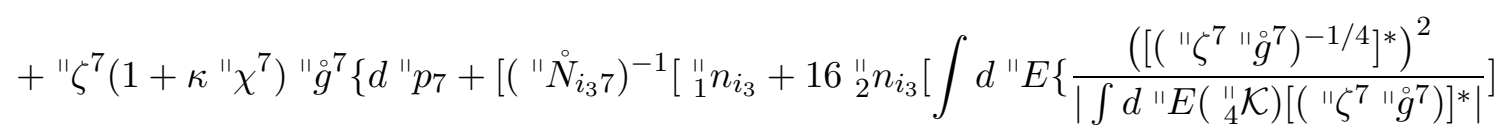

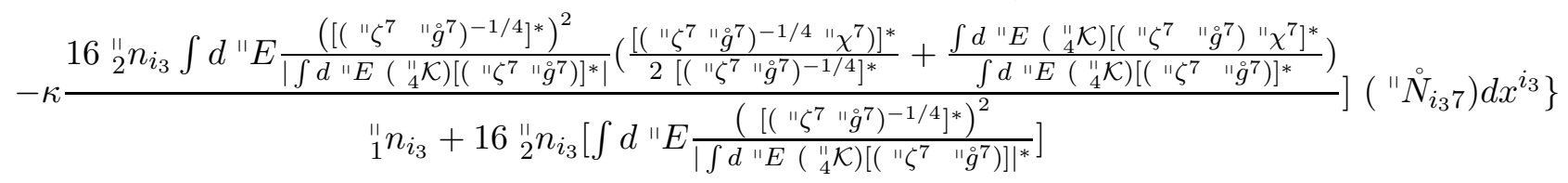

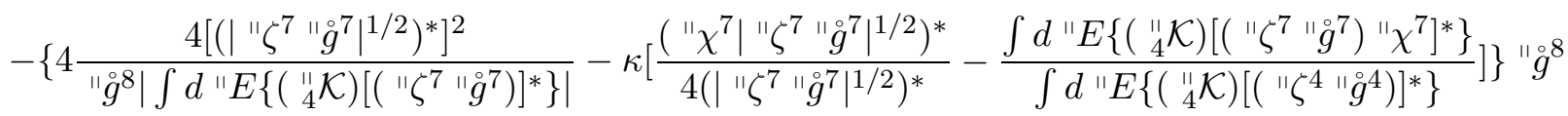

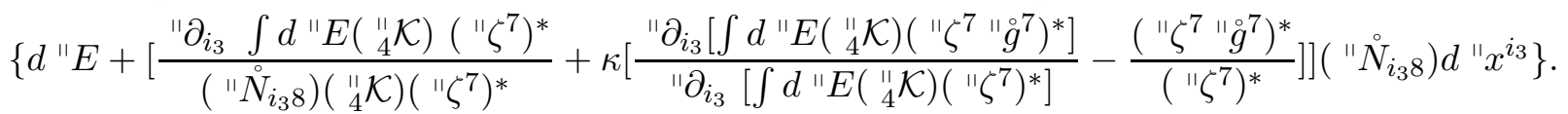


Using parametric generating functions and effective sources, we construct generic off-diagonal s-metric configurations with respective star deformations to symmetric and nonsymmetric metrics. Nevertheless, it is possible to define certain nonholonomic geometric conditions (they may involve integration functions) on $\eta$ - and/or $\kappa$ - polarization functions when the target quadratic line elements are diagonal. For other classes of phase space, or spacetime quasi-stationary metrics, we can impose such nonholonomic constraints via prescribed cosmological constants, when the effective sources are zero and the star deformed canonical Ricci s-tenor satisfies the conditions (62). Sections 5.4 and 5.5 in [40] contain respective details and a number of examples of diagonal and/or non-diagonal, effective/ pure vacuum solutions of associative and nonassociative phase space gravitational equations. Those s-metric can be taken as $\kappa^{0}$-approximation for solutions which can be star deformed to nonassociative dyadic gravitational configurations as we described appendix.

\section{References}

[1] R. Blumenhagen and M. Fuchs, Towards a theory of nonassociative gravity, JHEP 1601 (2016) 039; arXiv: 1604.03253

[2] P. Aschieri, M. Dimitrijević Ćirić, R. J. Szabo, Nonassociative differential geometry and gravity with non-geometric fluxes, JHEP 02 (2018) 036; arXiv: 1710.11467

[3] S. Vacaru, E. V. Veliev, and L. Bubuianu, Nonassociative geometry of nonholonomic phase spaces with star R-flux deformations and (non) symmetric metrics (under elaboration)

[4] S. Vacaru, On axiomatic formulation of gravity and matter field theories with MDRs and FinslerLagrange-Hamilton geometry on (co) tangent Lorentz bundles, arXiv: 1801.06444

[5] L. Bubuianu and S. Vacaru, Axiomatic formulations of modified gravity theories with nonlinear dispersion relations and Finsler-Lagrange-Hamilton geometry, Eur. Phys. J. C 78 (2018) 969

[6] A. Einstein, Einheitliche Fieldtheorie von Gravitation and Electrizidät, Sitzungsberichte der Preussischen Akademie Wissebsgaften, Mathematischn-Naturwissenschaftliche Klasse. (1925) 414-419; translated in English by A. Unzicker and T. Case, Unified Field Theory of Gravitation and Electricity, session report from July 25, 1925, pp. 214-419, arXiv: physics/0503046 and http://www.lrz-muenchen.de/ aunzicker/ae1930.html

[7] A. Einstein, A Generalization of the relativistic theory of gravitation, Ann. of Math. 46 (1945) 578-584

[8] L. P. Eisenhart, Generalized Riemann spaces, I. Proc. Nat. Acad. USA 37 (1951) 311-314

[9] L. P. Eisenhart, Generalized Riemann spaces, II. Proc. Nat. Acad. USA 38 (1952) 505-508

[10] J. W. Moffat, New theory of gravity, Phys. Rev. D 19 (1979) 3554-3558

[11] J. W. Moffat, Nonsymmetric gravitational theory, Phys. Lett. B 355 (1995) 447-452; arXiv: gr-qc/0411006

[12] J. W. Moffat, Nonsymmetric gravitational theory, J. Math. Phys. 36 (1995) 3722-3232; Erratum, 36 (1995) 7128

[13] J. W. Moffat, Noncommutative quantum gravity, Phys. Lett. B 491 (2000) 345-352; arXiv: hep-th/0007181

[14] S. Vacaru, Einstein gravity in almost Kaehler variables and stability of gravity with nonholonomic distributions and nonsymmetric metrics, Int. J. Theor. Phys. 48 (2009) 1973-2000; arXiv: 0806.3808 
[15] S. Vacaru, Einstein gravity, Lagrange-Finsler geometry, and nonsymmetric metrics, SIGMA, 4 (2008) 071, 29 pages; arXiv: 0806.3810

[16] S. Vacaru, Nonholonomic Ricci flows, exact solutions in gravity, and symmetric and nonsymmetric metrics, Int. J. Theor. Phys. 48 (2009) 579-606; arXiv: 0806.3812

[17] S. Vacaru, Exact solutions with noncommutative symmetries in Einstein and gauge gravity, J. Math. Phys. 46 (2005) 042503; arXiv: gr-qc/0307103

[18] S. Vacaru, Finsler black holes induced by noncommutative anholonomic distributions in Einstein gravity, Class. Quant. Grav. 27 (2010) 105003 (19pp); arXiv: 0907.4278 [math-ph]

[19] L. Bubuianu, K. Irwin and S. Vacaru, Heterotic supergravity with internal almost-Kaehler spaces; instantons for $\mathrm{SO}(32)$, or E8 x E8, gauge groups; and deformed black holes with soliton, quasiperiodic and/or pattern-forming structures, Class. Quant. Grav. 34 (2017) 075012; arXiv: 1611.00223

[20] L. Bubuianu and S. Vacaru, Deforming black hole and cosmological solutions by quasiperiodic and/or pattern forming structures in modified and Einstein gravity, Eur. Phys. J. C 78 (2018) 393; arXiv: 1706.02584

[21] L. Bubuianu and S. Vacaru, Black holes with MDRs and Bekenstein-Hawking and Perelman entropies for Finsler-Lagrange-Hamilton spaces, Annals of Physics, NY, 404 (2019) 10-38; arXiv: 1812.02590

[22] S. Vacaru and L. Bubuianu, Classical and quantum geometric information flows and entanglement of relativistic mechanical systems, Quantum Inf. Process, QINP 18 (2019) 376; arXiv: 1905.13015

[23] S. Vacaru, Geometric information flows and G. Perelman entropy for relativistic classical and quantum mechanical systems, Eur. Phys. J. C 80 (2020) 639; arXiv: 1905.12399

[24] C. W. Misner, K. S. Thorn and J. A. Wheeler, Gravitation (Freeman, 1973)

[25] S. W. Hawking and C.F. R. Ellis, The Large Scale Structure of Spacetime (Cambridge University Press, 1973)

[26] R. W. Wald, General Relativity (Universtiy of Chicago Press, Chicago, IL, 1984)

[27] D. Kramer, H. Stephani, E. Herdlt, and M. A. H. MacCallum, Exact Solutions of Einstein's Field Equations, 2d edition (Cambridge University Press, 2003)

[28] V. G. Kupriyanov and D. V. Vassilevich, Nonassociative Weyl star products, JHEP 1509 (2015) 102; arXiv: 1506.02329

[29] V. G. Kupriyanov, Non-associative star products and quantization of non-geometric backgrounds in string and M-theory, PoS CRFU2017 (2018) 200; arXiv: 1804.10161

[30] R. J. Szabo, An introduction to nonassociative physics, Published in: PoS CORFU2018 (2019) 100; arXiv: 1903.05673

[31] D. F. Kurdgelaidze, The foundation of nonassociative classical field theory, Acta Phys. Hung. 57 (1985) 79

[32] S, Okubo, Introduction to Octonion and other Non-associative Algebras in Physics (Cambridge Univ. Press, 1995) 
[33] C. Castro Perelman, The Noncommutative and Nonassociative Geometry of Octonionic Spacetime, Modified Dispersion Relations and Grand Unification, J. Math. Phys, 48 (2007) 073517

[34] C. Castro Perelman, Octonionic Ternary Gauge Field Theories Revisited, IJGMMP, (2014) 1450013

[35] C. Castro Perelman, R x C x H x O-valued Gravity as a Grand Unified Field Theory, Adv. Appl. Clifford Algebras 29 (2019) 22

[36] M. Günaydin, D. Lüst and E. Malek, Non-associativity in non-geometric string and M-theory background, the algebra of octonions, and missing momentum models, JHEP 1611 (2016) 027, arXiv: 1607.06474

[37] V. G. Drinfeld, Quasi-Hopf algebras, Alg. Anal. 1 N6 (1989) 114-148

[38] D. Mylonas, P. Schupp, and R. J. Szabo, Non-geometric fluxes, quasi-Hopf twist deformations and nonassociative quantum mechanics, J. Math. Phys. 55 (2014) 122301; arXiv: 1312.1621

[39] D. Mylonas, P. Schupp, and R. J. Szabo, Membrane sigma-models and quantization of non-geometric flux backgrounds, JHEP 09 (2012); arXiv: 1207.0926

[40] L. Bubuianu and S. Vacaru, Quasi-stationary solutions in gravity theories with modified dispersion relations and Finsler-Lagrange-Hamilton geometry, Eur. Phys. J. P 135 (2020) 148; arXiv: 1806.04500

[41] S. Vacaru, Noncommutative Einstein, almost Kaehler - Finsler and Quantum Deformations. Chapter 24 in: "The Algebraic Way. Space, Time and Quantum Beyond Peaceful Coexistence", ed. Ignazio Licata (World Scientific, Singapore, 2016), pp. 661-695

[42] R. Penrose and W. Rindler, Spinors and Space-Time. Vol. 1 (Cambridge University Press, 1984)

[43] R. Penrose and W. Rindler, Spinors and Space-Time. Vol. 2 (Cambridge University Press, 1986)

[44] Clifford and Riemann- Finsler Structures in Geometric Mechanics and Gravity, Selected Works, by S. Vacaru, P. Stavrinos, E. Gaburov and D. Gonta. Differential Geometry - Dynamical Systems, Monograph 7 (Geometry Balkan Press, 2006); www.mathem.pub.ro/dgds/mono/va-t.pdf and arXiv: gr-qc/0508023 [Part III is devoted to noncommutative Finsler-Lagrange gravity, strings and Clifford-Finsler gravity]

[45] S. Vacaru, Locally anisotropic gravity and strings, Ann. Phys. (NY), 256 (1997) 39-61; arXiv: gr-qc/9604013

[46] S. Vacaru, Superstrings in higher order extensions of Finsler superspaces, Nucl. Phys. B, 434 (1997) 590-656; arXiv: hep-th/9611034 